\title{
Intramural Visualization of Scroll Waves in the Heart
}

Dissertation

FOR THE AWARD OF THE DEGREE

DOCTOR RERUM NATURALIUM

submitted by

Jan Christoph

from Clausthal-Zellerfeld

Division of Mathematics and Natural SciEnces OF THE GEORG-August-University GÖTtINGEN 2014 



\section{Members of the Examination Board:}

Honorarprof. Dr. Stefan Luther (First Reviewer, Member of Thesis-Committee) Research Group Biomedical Physics Max Planck Institute for Dynamics and Self-Organization, Göttingen, Germany

Prof. Dr. Stephan Herminghaus (Member of Thesis-Committee)

Department of Dynamics of Complex Fluids

Max Planck Institute for Dynamics and Self-Organization, Göttingen, Germany

Prof. Dr. Sarah Köster (Member of Thesis-Committee)

Institute for X-Ray Physics

Georg-August-University Göttingen, Germany

Further Members of the Examination Board:

apl. Prof. Dr. Ulrich Parlitz

Research Group Biomedical Physics

Max Planck Institute for Dynamics and Self-Organization, Göttingen, Germany

Prof. Dr. Jens Frahm

Biomedizinische NMR Forschung GmbH

Max Planck Institute for Biophysical Chemistry, Göttingen, Germany

Prof. Dr. Eberhard Bodenschatz (Second Reviewer)

Department of Fluid Dynamics, Pattern Formation and Biocomplexity

Max Planck Institute for Dynamics and Self-Organization, Göttingen, Germany

Date of Submission: August 31st, 2014

Date of Oral Examination: October 13th, 2014 

I hereby declare that this thesis has been written independently and with no other sources and aids than quoted. 


\section{Contents}

1 Introduction 11

1.1 Spiral and Scroll Waves in Excitable Media . . . . . . . . . . . . . . . . . . 12

1.1 .1 Excitable Media . . . . . . . . . . . . . . . . . . . 12

$1.1 .2 \quad$ Spiral Waves . . . . . . . . . . . . . . . . . . . . . . . . . . . 14

1.1.3 Scroll Waves and Vortex Filaments _. . . . . . . . . . . . . 15

1.2 The Heart . . . . . . . . . . . . . . . . . . . . . . . . . . 17

1.2.1 Heart Anatomy . . . . . . . . . . . . . . . . . . . . . . 17

1.2.2 Cell Physiology and Function . . . . . . . . . . . . . . . 18

1.2.3 Myocardium: Functional Syncytium of Cells . . . . . . . . . . . . . 20

1.3 Heart Disease: Cardiac Arrhythmias . . . . . . . . . . . . . . . . . . 21

1.3.1 Cardiac Tachyarrhythmias: Tachycardia and Fibrillation . . . . . . . . . 21

1.3.2 Complex Wave Patterns underlying Cardiac Arrhythmias . . . . . . . . . . . 23

1.3.3 Clinical Relevance of Chaotic Wave Pattern . . . . . . . . . . . . . . . . 25

1.4 Intervention and Therapy . . . . . . . . . . . . . . . . . . 26

1.4.1 Cardioversion and Defibrillation . . . . . . . . . . . . . . . 27

1.4.2 New Approaches: Low-Energy Anti-fibrillation Pacing . . . . . . . . . . . . 27

1.4 .3 Ablation Therapy . . . . . . . . . . . . . . . . . . . 27

1.4.4 Other Therapy: Pharmacological Therapy, Cardiac Tissue Engineering and

Cell Therapy . . . . . . . . . . . . . . . . . . . 28

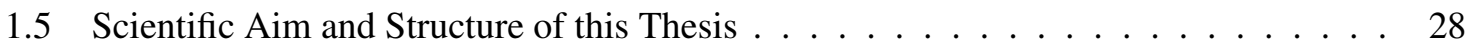

2 Mathematical Modeling of Cardiac Tissue 31

2.1 Cardiac Electrophysiology . . . . . . . . . . . . . . . . . 32

2.1.1 Single Cell and Ion Channel Dynamics . . . . . . . . . . . . . . . . . 32

2.1.2 Cell Syncytium and Spreading Waves of Electrical Excitation . . . . . . . . 33

2.1.3 Reaction-Diffusion Systems . . . . . . . . . . . . . . . . . . . . 34

2.1 .4 Excitable Media . . . . . . . . . . . . . . . . . . . . . . 34

2.1.5 Phenomenological Models of Cardiac Electrophysiology . . . . . . . . . . . 37

2.1 .6 Restitution . . . . . . . . . . . . . . . . . . . . . . 39

2.1 .7 Calcium Dynamics . . . . . . . . . . . . . . . . . . . 40

2.2 Cardiac Tissue Elasto-Mechanics . . . . . . . . . . . . . . . . . . . . . . . 40

2.2.1 Configurations of a Continuum Body . . . . . . . . . . . . . . . 41

2.2.2 Deformation and Strain . . . . . . . . . . . . . . . . . . . 42

2.2.3 Elastic Anisotropy of Myocardial Muscle Tissue . . . . . . . . . . . . . . . . . 46

2.2 .4 Stress and Constitutive Laws . . . . . . . . . . . . . . . . . . . . 46

2.2.5 Computational Modeling of Elasticity . . . . . . . . . . . . . . . 48 
2.3 Electromechanical Coupling . . . . . . . . . . . . . . . . . . . . . 50

2.3.1 Forward and Backward Electromechanical Coupling . . . . . . . . . . . 50

2.3.2 Elastic Anisotropic Active Stress Development and Elasticity . . . . . . . . 51

2.3.3 Computational Modeling of Electromechanically Coupled Wave Activity . . 52

3 Imaging of Electromechanical Wave Activity in the Heart 53

3.1 Optical Fluorescence Imaging $\ldots \ldots \ldots \ldots \ldots \ldots$

3.1 .1 Fluorescent Probes . . . . . . . . . . . . . . . . . . . . 55

3.1 .2 Imaging Hardware $\ldots \ldots \ldots \ldots \ldots 5$

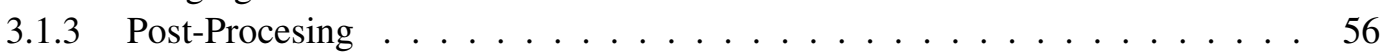

3.1 .4 Clinical Applications . . . . . . . . . . . . . . . . . . . . 56

3.2 Ultrasound: Echocardiography . . . . . . . . . . . . . . . 57

3.2 .1 Fundamentals . . . . . . . . . . . . . . . . . . . . 57

3.2.2 Imaging Modes: Two- vs. Three-Dimensional Imaging . . . . . . . . . . . . 57

3.2 .3 Clinical Applications . . . . . . . . . . . . . . . . . . . . . . . . . . 59

3.3 Other Imaging Techniques $\ldots \ldots \ldots$

3.3.1 Magnetic Resonance Imaging . . . . . . . . . . . . . . . . . . . 59

3.3.2 Computerized Tomography . . . . . . . . . . . . . . . . . . . 59

3.3.3 Transillumination and Optical Tomography . . . . . . . . . . . . . . . . 60

3.3.4 Electrode Recording . . . . . . . . . . . . . . . . . . . . . . . 60

3.3.5 Optical Coherence Tomography . . . . . . . . . . . . . . . . . . . . . . 60

3.3 .6 Other Imaging Techniques . . . . . . . . . . . . . . . . . . . . . . . . . . 64

3.4 Computational Techniques: Image Registration . . . . . . . . . . . . . . . 65

3.4.1 Correlation-based Motion Tracking . . . . . . . . . . . . . . . 65

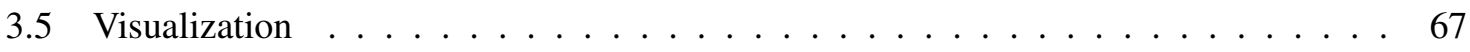

4 Computational Model of Heart Tissue with Mechano-Electric Coupling 6

4.1 Modeling of Electrophysiological Excitation Patterns . . . . . . . . . . . . 70

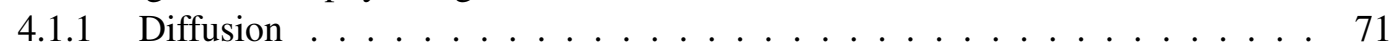

4.1 .2 Initial Conditions . . . . . . . . . . . . . . . . . 72

4.2 Elasto-Mechanical Model . . . . . . . . . . . . . . . . . . . . . 72

4.2.1 Simulation of Elasticity using Discrete Particle System . . . . . . . . . . . 72

4.2 .2 Verlet Integration . . . . . . . . . . . . . . . . . . . . 76

4.3 Actively Contracting Myocardial Tissue . . . . . . . . . . . . . . . 78

4.4 Realistic Heart Modelling . . . . . . . . . . . . . . . . . . . . . . . . . 78

$4.4 .1 \quad$ Rabbit Heart Model . . . . . . . . . . . . . . . . . . . . . . . 79

5 Electromechancial Wave Pattern Reconstruction $\quad 81$

5.1 Electromechanical Coupling and Wave Hypothesis _ . . . . . . . . . . . . . 82

5.1 .1 Deformation Measures . . . . . . . . . . . . . . . . . . . 84

5.2 Planar Electromechanical Wave Propagation . . . . . . . . . . . . . . . . 85

5.2.1 Muscle Fiber Anisotropy . . . . . . . . . . . . . . . . . . . . . . . . 87

5.2.2 Wave Front Detection from Dynamic Features of Elasticity . . . . . . . . . 88

5.3 Electromechanical Spiral and Scroll Wave Patterns . . . . . . . . . . . . . . . 88

5.3.1 Two-dimensional Spiral Wave Patterns . . . . . . . . . . . . . . . . 88

5.3.2 Three-dimensional Scroll Wave Patterns . . . . . . . . . . . . . . . . . . . 92

5.3.3 Reconstructing Wave Front and Wave Pattern Topology . . . . . . . . . . . . 97 
5.4 Electromechanical Scroll Vortex Wave Filaments . . . . . . . . . . . . . . . . . 101

5.4.1 Detection and Reconstruction of Filament Structure . . . . . . . . . . . . . . 102

5.4.2 Properties of Electromechanical Filaments . . . . . . . . . . . . . 105

5.4 .3 Emulation of Imaging Experiment . . . . . . . . . . . . . . . . . 108

5.5 Inverse and Electromechanical Wave Imaging . . . . . . . . . . . . . . . . . . . 109

5.5.1 Elastic Deformation Tracking . . . . . . . . . . . . . . . . 111

5.5.2 Elastic Deformation Tracking tested with Synthetic Data . . . . . . . . . . 115

5.5.3 Electromechanical Spiral Wave Tracked in Experimental Data of Engineered

Cardiac Cell Culture . . . . . . . . . . . . . . . . . . 117

6 Intramural Scroll Wave Imaging during Ventricular Tachycardia and Fibrillation 119

6.1 Experimental Design and Imaging Setup . . . . . . . . . . . . . . . . . . 120

6.2 Electromechanical Coupling Hypothesis . . . . . . . . . . . . . . . . . . . . 124

6.3 Tissue Preparation and Arrhythmia Control . . . . . . . . . . . . . . . . . . . 124

6.4 Fluorescence Imaging . . . . . . . . . . . . . . . . . . . . . . 125

6.4.1 Optical Imaging Setup and Data Acquisition . . . . . . . . . . . . . 125

6.4.2 Staining and Image Properties of Fluorescence Video Data . . . . . . . . . . 126

6.4.3 Motion Tracking and Motion Artifact Removal . . . . . . . . . . . . . . . 132

6.4 .4 Post-Processing of Fluorescence Imaging Data . . . . . . . . . . . . . . . . 140

6.5 Ultrasound Imaging . . . . . . . . . . . . . . . . . . . . . . 141

6.5.1 Imaging Setup and Data Acquisition . . . . . . . . . . . . . . . . . . 141

6.5.2 Imaging Configuration . . . . . . . . . . . . . . . . . . . . . . 143

6.5.3 Elastic Deformation Tracking . . . . . . . . . . . . . . . . . . . 143

6.6 Electromechanical Scroll Wave Imaging during Ventricular Tachycardia and Fibril-

lation . . . . . . . . . . . . . . . . . . . . . 145

6.6.1 Spiraling Electromechanical Activity on Ventricular Surface . . . . . . . . . 146

6.6.2 Electromechanical Scroll Wave Activity inside Ventricular Wall . . . . . . . 151

7 Discussion $\quad 161$

7.1 Summary of Results . . . . . . . . . . . . . . . . . . . . . . 161

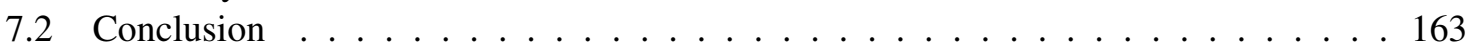

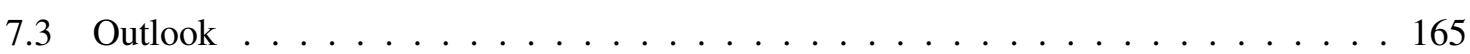

7.3 .1 Immediate . . . . . . . . . . . . . . . . . 165

7.3 .2 Intermediate . . . . . . . . . . . . . . . . . 166

7.3.3 Long-Term: Translation to Clinical Applications . . . . . . . . . . . . 167

$\begin{array}{ll}\text { A Physical Units and Abbreviations } & 189\end{array}$

$\begin{array}{lll}\text { B Tables } & 191\end{array}$ 


\section{Contents}


Chapter 1. Introduction

\section{Chapter 1}

\section{Introduction}

Despite extensive research, the fundamental biophysical mechanisms underlying ventricular fibrillation - one of the deadliest diseases in the world - remain poorly understood. Ventricular fibrillation is an acute and highly lethal state, in which the heart's proper pumping function is cessated by undesired, self-sustained fibrillatory electrical activity inside the heart muscle. As a result, the heart loses its capability to propel oxygen-rich blood through the body, which can lead to death within few minutes if untreated. The abnormal electrical activity often arises spontaneously, making the disease difficult to predict and treatment challenging. During the normal heart rhythm, the electrical activity is re-initiated in each heart beat by the heart's internal pace-making system and spreads through the cardiac muscle to trigger an orderly, coordinated contraction. The cardiac muscle consists of billions of contractile cells, cardiomyocytes, which contract upon electrical excitation. The electrical activity is constituted by nonlinear waves of electrical excitation, which propagate through the cardiac muscle tissue and cause the subsequent activation of the cells. During the normal heart rhyhtm, the spatial-temporal organization of the electrical wave pattern proves to be highly coherent, maintaining a synchronous, orchestrated activation of the cells, and producing a uniform, strong contraction of the cardiac muscle. However, during heart rhythm disorders, the electrical wave pattern appears to be highly disorganized and the loss of synchrony of the mechanical activation of the cells results, on the tissue level, in a disruption of the uniform contraction of the cardiac muscle. Generally, heart rhythm disorders, or cardiac arrhythmias, are attributed to the abnormal electrical activity, as the electrical activity is considered to govern the contractile behavior of the heart and, furthermore, the irregular heart beat can be directly associated with the disordered electrical wave pattern. Different signatures of the electrocardiogram, which can be associated with different stages and types of heart rhythm disorders, can also be associated with different patterns of the electrical activity.

Understanding the nature of the underlying abnormal electrical activity is thought to be key to the development of therapeutical strategies in treating cardiac arrhythmias. Research focusing on seeking out the underlying molecular, biophysical processes on the cellular and subcellular level has led to major insights regarding arrhythmogenic factors predisposing the heart to arrhythmias. However, the level of detail in the acquired knowledge about the subcellular processes involved in cardiac disease is contradicted by a lack of understanding of how the molecular mechanisms manifest in a large, macroscopic electrical pattern in a multi-cellular substrate that involves many billions of cardiac cells. The complex dynamical phenomena that arise in such large and complicated systems are typically studied from the perspective of complex and dynamical systems theory and are associated with the emergence of spatial-temporal complexity and pattern formation phenomena in non-equilibrium systems. Car- 
diac research has since been determined by an interdisciplinary community of researchers consisting of physicians, electrophysiologists, biologists, mathematicians, physicists, computer scientists and biomedical engineers. The understanding of cardiac arrhythmias has advanced with each of the disciplines contributing their expertise and, in particular, it was one of the great achievements of the field when mathematicians and physicists were able to identify the collective behavior of the many cells and to describe it using mathematical equations. Specifically, the field of theoretical physics, and more specifically nonlinear dynamics, ${ }^{228}$ has contributed significantly to the understanding of the behavior of the heart during cardiac arrhythmias by providing mathematical and computational models that are able to explain the macroscopic appearance of the electrical wave pattern underlying ventricular fibrillation. However, recent advances of the field have been hindered in large part due to lacking imaging technology capable of providing panoramic in-depth visualizations of the electrical pattern during cardiac arrhythmias inside the heart muscle and consequently providing direct experimental evidence of the postulated theoretical models. To date, experimental proof is limited to observations of the electrical pattern on the surface of the heart.

This work aims to contribute to a better understanding of the abnormal wave pattern underlying cardiac fibrillation and provides a novel approach for its visualization within the cardiac muscle. This chapter introduces the basic concepts of the field, points out its challenges and ends with a formulation of the scientific aim and an outline of the structure of this thesis. The topics covered in this thesis lay at the interface between physics and medicine. Accordingly, in the following sections they are approached from either side, first from the perspecive of physics and then from the perspective of medicine, to be able to relate the two and to come to an understanding of the fundamental physical phenoma that can be observed in the heart during cardiac fibrillation.

\subsection{Spiral and Scroll Waves in Excitable Media}

Spiral waves are known to occur frequently in nature. ${ }^{41,42,73}$ They belong to a family of pattern forming phenomena associated with reaction-diffusion kinetics, see also section 2.1.3 in chapter 2 , and occur in many physical, chemical and biological systems, see also figure 1.2. Spiral wave patterns form dynamically in spatially extended excitable systems due to intrinsic properties of the constituents of the system, that allow the propagation of nonlinear waves of excitation, see also section 2.1.4. Such systems belong to a specific class of dynamic systems referred to as excitable media ${ }^{42}$ From a dynamical systems theory perspective, the heart is considered to be an excitable medium, see section 1.2. In the following, spiral and scroll waves are introduced as an abstract concept, explaining - without mathematical detail - the underlying physical mechanisms that lead to the respective pattern forming behavior of the abnormal electrical pattern in the heart during cardiac arrhythmias. The concept can similarly be applied to explain the behavior of various other systems. A more formal introduction to excitable media is given in chapter 2 .

\subsubsection{Excitable Media}

An excitable medium is constituted of excitable small units or compartments which are coupled to each other. The coupling is important as it allows the distribution or exchange of excitation among the compartments and through the system. Generally, the connectivity among the units can take on any form but typically, and specifically for quasi-continuous excitable media, it is the case that units are connected to their nearest neighbours. Nearest-neighbour coupling results in the normal 

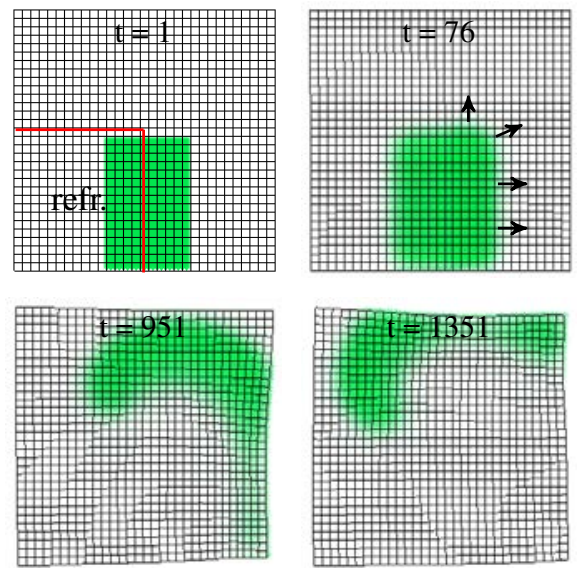
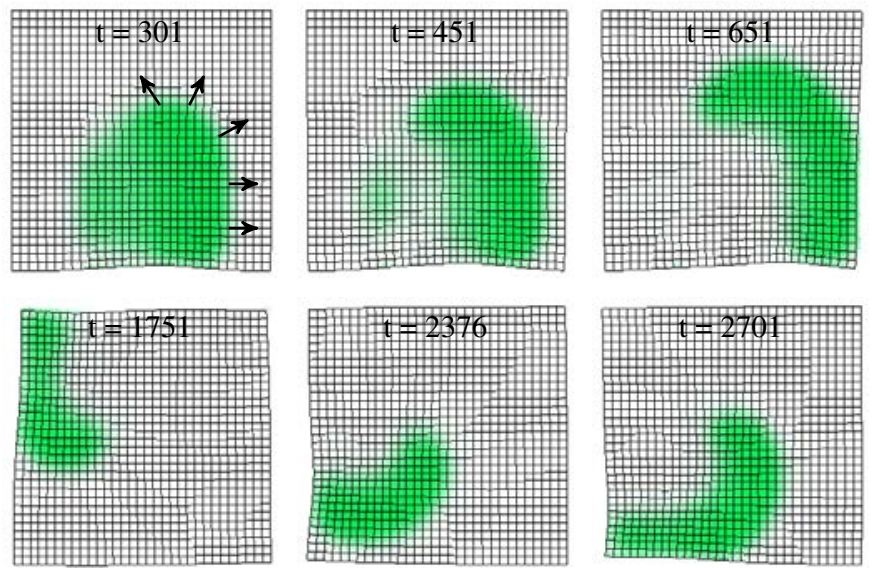

Figure 1.1: Spiral wave formation in excitable medium: the medium consists of small units which are all coupled to their neighbouring units. As each unit can be driven from a resting state (white) into an excited state (green) and the excitation can be passed on to the neighbouring units, the medium supports the spread of nonlinear waves of excitation. A spiral wave can arise from one planar wave of excitation that is being blocked on one side (refractory area within lower left corner of medium) and that retains one open end (here at the center of the medium). Because the lower left corner is in the beginning of the sequence in a refractory state $(t=1$, then decays) and blocks all excitation, the excitation can only spread towards the right side of the medium to form a counterclock-wise rotating spiral. Here, the two-dimensional medium undergoes elastic deformations as the excitation triggers contraction. The spiral wave rotates around its own tip and presents a spatial-temporal pattern that can persist theoretically indefinitely.

diffusive spread of excitation through the system, which retains the character of a normal diffusive process and leads to wave phenomena. The reaction-diffusion character of excitable media is then completed by the reaction of the local kinetics. By default, the individual units are in a resting state but can be excited by external sources or neighbouring units that provide a sufficiently large stimuli that pushes the units away from the resting state into an excited state. Each unit is only able to stay in the excited state for a limited amount of time and subsequently enters a refractory state in which it similarly stays only for a limited amount of time before it finally reaches the resting state again. This cycle can be repeated at any time, provided that each unit experiences a sufficiently large stimuli and holds intrinsic energy to be able to undergoe the cycle. The excitatory state of each individual unit is an unstable state which can be accessed from a resting state and can only be triggered by external sources or neighbouring units providing a sufficiently large stimuli that pushes the units away from the resting state to start the cycle. The following excitation can only be maintained for short periods of time and the unit returns, after a refractory period in which the unit can not be stimulated any longer, to its resting state. During the excited state, the unit can transmit parts of its excitation to its neighbours. As a conquence, we obtain cyclic dynamics that support propagating waves of excitation in the system, where all waves of excitation are followed by waves of refractoriness. Systems showing such behavior can exhibit spiral waves.

The most intuitive example of an excitable system is a forest fire. Its individual small compartments are trees and its diffusive process is that the fire can jump from tree to tree. When the forest is lit on fire at one side, the fire is going to burn from one side to the other side of the forest leaving behind black, burnt forest. If the forest is large enough, one will observe three different phases: green forest, 


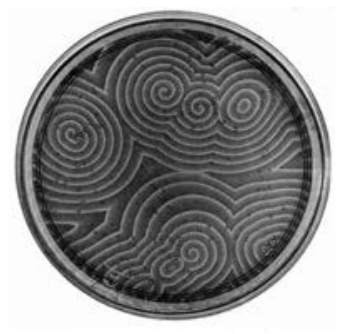

(a)

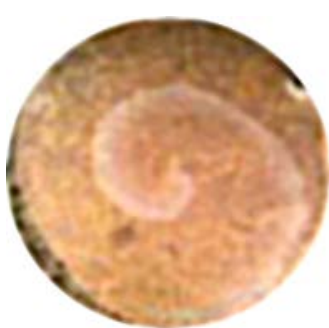

(b)

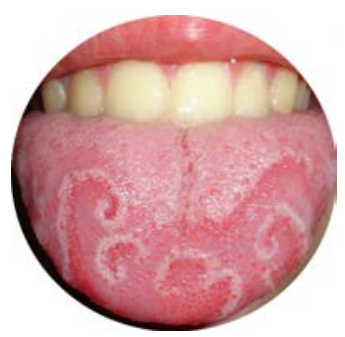

(c)

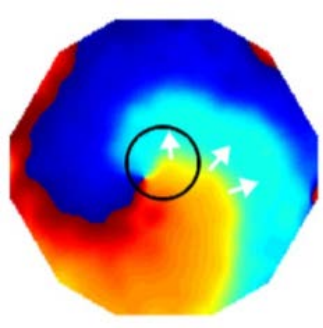

(d)

Figure 1.2: Spiral wave patterns in different biological and chemical systems: (a) Belousov-Zhabotinsky chemical reaction $^{15}$ (b) neuronal depression wave in chicken retina, image courtesy of Yu et al., ${ }^{210}$ (c) inflammatory disease called geographic tongue $e^{235,237}$ and (d) action potential spiral wave in cardiac monolayer cell culture, image courtesy of Lin et al. ${ }^{146}$

a fire front and black, burnt forest. The fire front is the nonlinear wave of excitation and it is fueled by the trees as the fire requires energy provided by the wood in order to exist and to be able to propagate. When starting the fire from a site located at the center of the forest, one will observe a circular ring of fire spreading outwards towards the boundaries of the forest. The fire front can not simply turn around or start burning into directions from where it came from as it requires new, dry wood to burn. As it used up all its fuel in areas behind it, it simply would extinct. As discussed previously, this directed propagation is an important feature of an excitable medium. It leads, in the case of a ring of fire, to an extinction of the fire at the boundaries, but more importantly, it may lead to the formation of a spiral wave. That is, there are instances where the fire can turn around and persist theoretically forever.

\subsubsection{Spiral Waves}

Spiral waves form due to a so-called reentrance of excitation, see also following section. The formation of a spiral wave is depicted in figure 1.1. In terms of the forest fire, one could imagine a forest that was so large that the burnt forest was given enough time to regenerate and regrow before the fire - that in the meantime still burned elsewhere in the forest - could return. The fire then had new ground to burn and could potentially elicit the same part of the forest twice. Now, this requires that the fire has the opportunity to return back to its origin, to circumvent the black, burnt forest that it left behind. Such a situation can be prepared as follows, see also figure 1.1: If one were to cut a street into a square-shaped forest to act as a fire break and lays this street from the center of one edge to the centre of the forest and lights the trees on one side of that street on fire, a straight line of fire would burn away from the street into the forest. As the street acts as a fire break, the forest on the other side of the street would remain unaffected by the fire. However, as the fire front propagated away from the street and burnt around the tip of the fire break it would start to bend and soon take on the shape of a spiral as shown in figure 1.1. Figure 1.1 shows a computer simulation of an elastic excitable medium, see section 4.3 in chapter 4 . The green wave indicates excitatory activity that can spread from node to node of the grid. The first image shows the initially prepared situation, that corresponds to the situation described above. The simulation domain is set to the 'excited' state (green) along a straight line from the bottom center to the central point of the medium. Right next to the line (here not visble), on its left side, the simulation domain is set to the 'refractory' state. This line corresponds to the fire break. Accordingly, the green wave starts to spread, but decays in the following images towards the left side. On the right side, however, the wave can spread freely and as it evolves it starts to curl, 
to form a spiral wave that circulates around a rotational center. ${ }^{13,15}$ It is important to note that the spiral wave forms dynamically and does not have to rotate necessarily around an obstacle. Instead, the rotational center is provided by the spiral's internal dynamic behavior. Spiral waves organize in a self-organized fashion around rotational centers, which are often referred to as phase singular points. In a real forest, the above described behavior is a very unlikely scenario as the two time-scales on which the fire burns and the forest regrows are very different. However, in other systems these two time scales may very well be similar and therefore enable this behavior. For instance, in a cardiac muscle cell the action potential duration and the refractory period are both in the order of a few hundred milliseconds, see following section. Therefore, the intrinsic propeties of cardiac muscle cells support the formation of nonlinear waves of excitation and spiral waves.

Many more systems are known to exhibit spiral wave patterns. ${ }^{41,42,73}$ The most prominent system is the bromate-malonic acid reagent or Belousov-Zhabotinsky ${ }^{15}$ chemical reaction, see figure 1.2(a). The reaction forms spiral wave patterns in the petri-dish and is often used for the illustration of spatial-temporal chaos in non-equilibrium systems. Spiral waves also occur during the oxidization process of platinum on the surface of the substrate. ${ }^{32}$ The slime mold dictyostelium discoideum exhibits cAMP spiral waves during the transition from a collection of unicellular amoebae into a multicellular organism in search for nutrition. ${ }^{30}$ Protein waves that provide a geometry-sensing mechanism on the cellular membrane exhibit spiral wave patterns on microstructured membranes. ${ }^{219}$ Fertilizing Xenopus oocyte calcium waves exhibit spiral wave patterns ${ }^{36}$ as well as glial calcium waves in cortical tissue culture. ${ }^{64}$ Spiral wave patterns occur in neuronal tissue such as the mammalian cortex ${ }^{100,189}$ or the retina, ${ }^{22}$ see figure $1.2(\mathrm{~b})$, where spiral wave-shaped spreading depression waves were reported to cause macular degeneration. ${ }^{210}$ Spiral waves may occur on the human tongue during a benign medical condition named geographic tongue, in which papillae on the surface of the tongue degenerate due to a slowly expanding inflammation, ${ }^{235}$ see figure 1.2(c). Lastly, spiral waves can be observed in monolayer cardiac cell culture preparations ${ }^{136,146,154}$ when visualizing, for instance, the action potential wave activity using fluorescence imaging.

\subsubsection{Scroll Waves and Vortex Filaments}

In three spatial dimensions, the spreading of nonlinear waves of excitation can take on the form of planar or spherical waves as compared to linear or circular-shaped waves in two dimensions. Also highly complicated wave shapes and patterns are possible. Scroll waves ${ }^{19,23,27,28}$ are the three-dimensional representatives of spiral waves, see figure 1.3. They form similarly as spiral waves dynamically around centers of rotation, which correspond in three-dimensional space to vortex filaments. ${ }^{24}$ These scroll vortex wave filaments can be arbitrarily shaped curves in space. The simplest form of a scroll wave is a spiral wave that repeats along its rotational axis, as shown in the upper panel of figure 1.3. The geometric shape of this scroll wave is very similar to a rolled sheet of paper. The scroll wave rotates around the central line, which is a line of phase singularity or the core region of the scroll wave or its scroll vortex wave filament respectively. The vortex filament translates to the spiral wave tip in two spatial dimensions. Scroll waves can be skewed, bend and take on many different shapes depending on the dynamical properties of the substrate supporting the activity. Accordingly, scroll wave filaments can be curved and can generally take on any shapes in three-dimensional space. ${ }^{52,166}$ Their evolution similarly depicts the behavior of the scroll wave. ${ }^{125}$ For instance, a ring-shaped filament corresponds to a scroll wave ring or focus, ${ }^{140,157}$ as depicted in the lower panel of figure 1.3. The interaction of multiple scroll waves with each other can lead to complicated wave patterns, which are described to be chaotic and turbulent, ${ }^{28,52,203}$ but also intermittently self-organizing. ${ }^{112}$ 


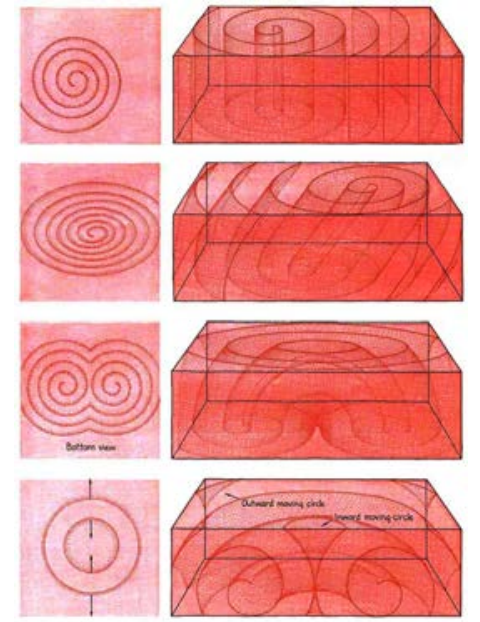

(a)

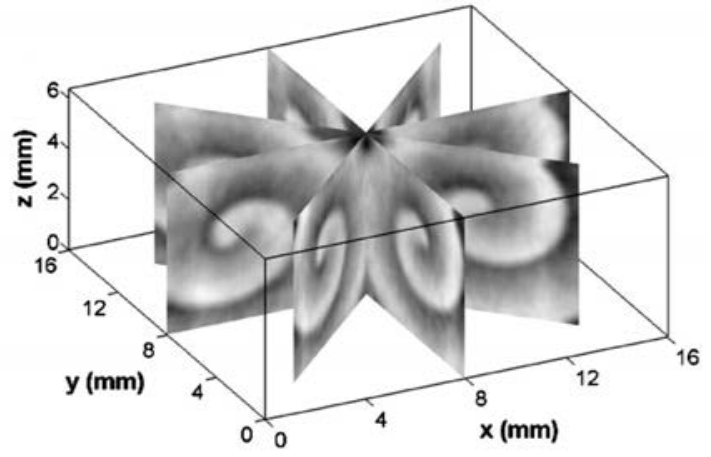

(b)

Figure 1.3: Scroll waves in three-dimensional excitable media: rotating waves of nonlinear excitation forming spiral wave-like shapes. (a) Seminal sketches of Winfree in 1978 of scroll waves and scroll wave rings (lower panel). (b) Three-dimensional reconstruction of a scroll wave ring in BelousovZhabotinsky chemical reaction using optical tomography. Figure courtesy of T. Bansagi and $O$. Steinbock, ${ }^{157} 2008$.

(g)

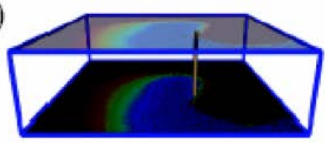

(j)

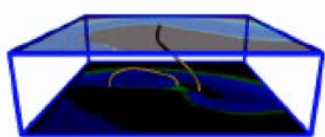

(m)

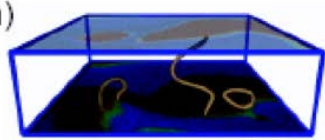

(h)

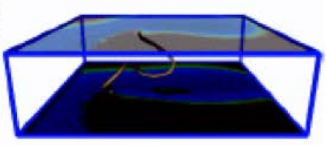

(k)

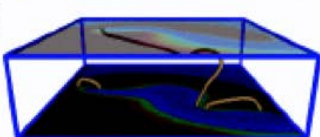

(n)

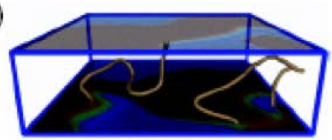

(i)

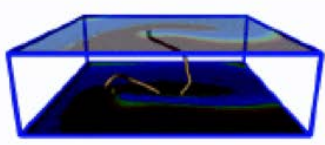

(I)

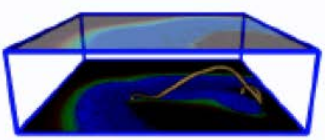

(o)

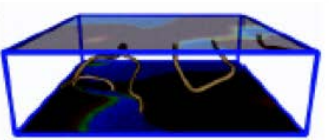

Figure 1.4: Vortex filaments indicating the organizational centers of scroll waves in computer simulations. Scroll wave filament interaction is conjectured to be responsible for the onset and emergence of turbulent electrical wave activity during fibrillation inside the cardiac muscle. Here, multiple filaments emerge from one unstable filament at the beginning of the sequence. Figures courtesy of Cherry et al. ${ }^{166}$ 
However, also a single scroll wave can evolve into complicated chaotic spatial-temporal patterns. ${ }^{84}$ Generally, vortex filament instabilities ${ }^{69,85}$ and interactions ${ }^{158}$ were found to be responsible for the onset and perpetuation of self-sustaining spatial-temporal disorder in excitable media. Crucial for the emergence of spatial-temporal disorder is whether a filament will shrink or expand, which is often referred to as filament tension. ${ }^{43}$ Negative filament tension corresponds to a filament that is growing in length. Accordingly, negative filament tension increases the likeliness of filament interactions and self-interactions and promotes the emergence of spatial-temporal chaos in excitable media. Figure 1.4 shows the break-up of a scroll wave due to negative filament tension. The scroll wave filament grows and eventually interacts with the medium boundaries or with itself and either way breaks up into multiple filaments, which in turn can lead to a cascade of filament and scroll wave rotor break-ups. Scroll vortex wave filaments can either be closed-loops within the volume of the excitable medium or they attach with both their ends to the surfaces of the medium. ${ }^{158}$ Accordingly, on the surface, the filaments exhibit phase singular points, which are the organizing centers of rotating patterns, which often resemble spiral wave-like shapes. Likewise, filaments cause the observation of pairwise creation and annihilation of phase singularities on the surface. Scroll wave and vortex filament dynamics are and were intensively studied in the chemical Belousov-Zhabotinsky reaction, ${ }^{140,157,226}$ because the translucent chemical substrate allows to use optical tomography techniques to capture the volumetric wave pattern, see figure 1.3(b).

\subsection{The Heart}

The heart is the central organ of our body. It is the first organ to begin development in the very early stages of the formation of the human body. It forms out of a tubular-shaped assembly of contractile cells, that loops and later septates into four chambers to become the heart-shaped organ as we know it. The heart beats approximately 120,000 times per day, more than 40 million times per year and ideally more than 3 billion times in a life-time. It pumps approximately 7, 000 liters of blood through the human body in one day.

\subsubsection{Heart Anatomy}

The heart is a complex system of muscles, valves and chambers, see figure 1.5. The heart muscle consists of billions of contractile muscle cells, cardiomyocytes, which contract on electrical excitation. Together with the heart's geometry and a system which controls and spreads excitation and maintains a coordinated excitation of the myocytes, pulsatile pumping is achieved. The human heart has a left and a right chamber, the ventricles, with each chamber having its own atrium. The valves in between ventricles and atria and in between ventricles and pulmonary artery and aorta maintain the directed blood flow. The atria fill with blood when the cardiac muscle releases. The ventricles receive blood from the atria and finally supply the main pumping force. The right ventricle propels oxygen-poor blood through the pulmonary circulation and the left ventricle propels oxygen-rich blood through the peripheral circulation. ${ }^{96,180}$ The walls of the ventricles and atria are composed of myocardium, which is the heart's muscle responsible for performing the contraction. Myocardium is an excitable, heterogeneous tissue which hosts billions of cardiomyocytes. Electrical excitation can spread in myocardium and excite all its cardiomyocytes. Each cardiomyocyte contracts on electrical excitation. Electrical excitation is initiated and conducted by an electrogenic system, composed of sinoatrial and atrioventricular nodes, His bundle and the heart conduction and Purkinje system, which generates rhythmical excitation and conducts and spreads this excitation rapidly into the myocardium. ${ }^{68,123}$ 


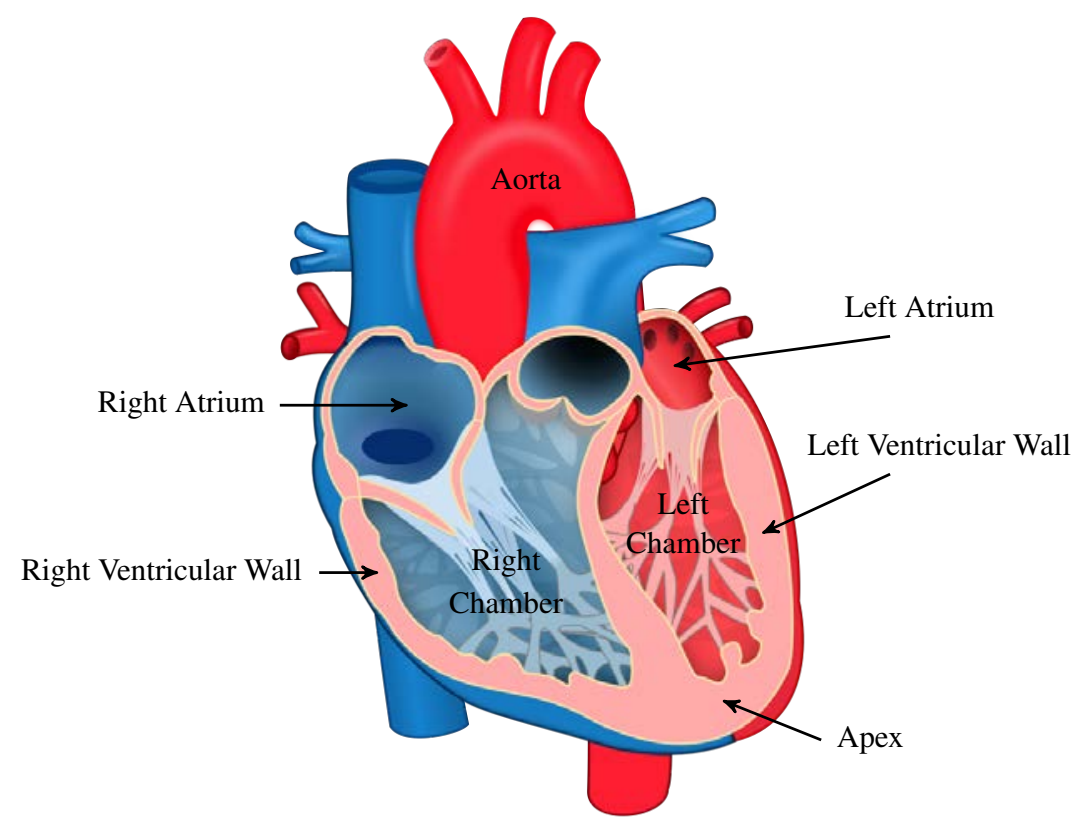

Figure 1.5: Anatomical picture of the heart: complex geometric structure of valves and chamber for pulsatile pumping of blood through the body. The cardiac muscle propels blood from four chambers, with the largest chamber, the left ventricle, propelling oxygen-rich blood through the peripheral circulation (the body). Image reproduced from Wikimedia Commons. ${ }^{236}$

The Purkinje system consists of pacemaker cells, that are self-excited, oscillating cells which are concentrated in nodes at specific regions in the heart, and a conductive system composed of conducting fibers. The excitation, transmitted from the nodes into the myocardium, arrives under normal physiological conditions at almost all locations in the ventricles at the same time, causing all portions of the muscle to begin contracting at the same time. The atria receive the excitation earlier, ensuring that they empty before the ventricles start to contract. In addition, the heart is supplied with sympathetic and parasympathetic nerves, which can have a direct influence on the excitation dynamics of the Purkinje system, as well on the myocardium directly. The cardiac muscle is infused with a dense vascular system. Its blood vessels and capillaries are local discontinuities of the conductive myocardium. In particular, the heart supplies itself with blood via its coronary arteries. The inner and outer layer of the heart's wall are the endocardium and epicardium, respectively. The heart is surrounded by the pericardium, a double-walled sac, which protects the heart and anchors its surrounding structures. ${ }^{96,180}$

\subsubsection{Cell Physiology and Function}

Biological cells possess membranes surrounding the cells interior, separating the intracellular from extracellular space. ${ }^{123}$ Cellular membranes play a pivotal role for the emergence of biological function and life in general. Extracellular space and intracellular space are both filled with an aqueous medium. This fluid contains concentrations of ions, which stem from dissolved salts and are capable of diffusing freely from place to place. The diffusion of ions in between extracellular and intracellular space is regulated by the membrane of the cell, as it is permeable either passivley or actively for certain ions. The cellular membrane embeds numerous individual channels which are permeable 
Chapter 1. Introduction

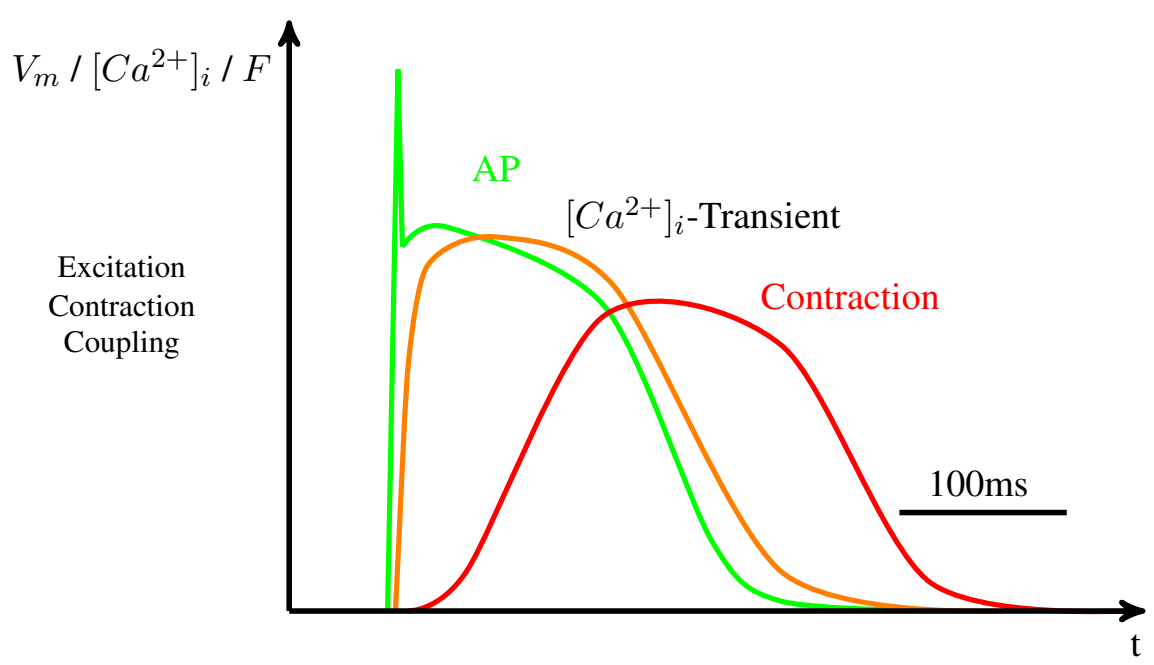

Figure 1.6: Excitation-contraction coupling in cardiac cell: action potential (AP, green curve) triggers release of intracellular calcium $\left[\mathrm{Ca}^{2+}\right]_{i}$ (orange curve), which in turn fuels contraction of cardiomyocyte (red curve). Figure replication from Bers et al., ${ }^{83} 2002$.

for a specific ion and which each have their own properties and criteria to let a specific ion pass. The most important ions, with respect to ion dynamics in cardiac tissue, are Calcium $\mathrm{Ca}^{2+}$, Sodium $\mathrm{Na}^{+}$, Potassium $\mathrm{K}^{+}$and Chloride $\mathrm{Cl}^{-}$ions, each of which match with either one specific channel or possibly a variety of channels. ${ }^{68}$ As these channels regulate an in- and outflux of ions they create a very complex interplay of fluxes which alter the ion concentrations in extracellular and intracellular space. The intracellular ion concentration is usually determined by the presence of $\mathrm{K}^{+}$ions and large protein anions, whereas the extracellular ion concentration is determined by the presence of $\mathrm{Na}^{+}$and $\mathrm{Cl}^{-}$ions. An increased amount of ions present in one space produces a net electrical charge, leading to an electrical potential or a voltage difference in between opposing spaces, that is across the membrane in between extra- and intracellular space. This is referred to as the transmembrane potential. For instance, in intracellular space, $K^{+}$ions and large protein anions neutralize their electrical charges. However, due to their high concentration and the specific selectivity of the cell membrane, $K^{+}$ions leave the cell driven by diffusion. As a result, a transmembrane potential builds up. Each cell has an equilibrium membrane potential in which diffusion forces and opposing forces of the electrical potential balance out. The equilibirum membrane potential $V$ of a cell is given by the Nernst-equation:

$$
V=\frac{R \cdot T}{e \cdot z \cdot N_{A}} \ln \left(\frac{c_{e}}{c_{i}}\right)
$$

in which $c_{i}$ and $c_{e}$ are the ion concentrations in intracellular and extracellular space respectively, $R$ is the universal gas constant, $T$ is the temperature, $e$ is the unit charge, $z$ the degree of ionization and $N_{A}$ is the Avogadro constant. There are excitable and non-excitable cells in the body. Cardiomyocytes are excitable cells, with the property that their membrane potential can vary highly dynamically over time. Their cell membrane features ion channels which have a sensitivity to changes in the transmembrane potential. When the membrane potential changes slightly, the ion channel dynamics of the cell can change dramatically. The resting or equilibrium membrane potential of a cardiomyocyte is about $-80 \mathrm{mV}$. If the membrane potential is altered externally, for instance by an applied electrical field 
or ionic charges diffusing into the cell from neighbouring cells, over a specific threshold of about $-70 \mathrm{mV}$, the cell experiences a cycle of rapid in- and out-streams of ions. As a result the membrane potential varies over time. This cycle is referred to as an action potential. The shape of the action potential can be seen in figure 1.6.

At first, fast membrane potential sensitive $\mathrm{Na}^{+}$ion channels open and leave positively charged $\mathrm{Na}^{+}$ into the cell. $\mathrm{Na}^{+}$ions are highly concentrated in the extracellular space. At the same time the permeability of the membrane for $\mathrm{K}^{+}$ions decreases and the membrane potential is driven towards the equilibrium potential of the $\mathrm{Na}^{+}$ions which is about $+60 \mathrm{mV}$. Within $1 \mathrm{~ms}$ the membrane potential has risen to about $+30 \mathrm{mV}$. This fast upstroke is typical for the shape of an action potential. It is referred to as the deporalization of the cardiac cell. The membrane potential is then held at a high level for a few hundred milliseconds. As the $\mathrm{Na}^{+}$ion channels are closing, calcium ion channels, which are activated more slowly than $\mathrm{Na}^{+}$ion channels, open and leave $\mathrm{Ca}^{2+}$ ions into the cell. While the diffusion of $\mathrm{K}^{+}$ions out of the cell remains low, the intracellular calcium ion concentration $\left[\mathrm{Ca}^{2+}\right]_{i}$ rises and keeps the membrane potential on a plateau, see figure 1.6. The diffusion process would otherwise initiate a repolarization of the cell. It is only after a phase of about $200 \mathrm{~ms}$ that repolarization sets in. Slowly, the permeability of the membrane for $\mathrm{K}^{+}$ions returns and the $\mathrm{Ca}^{2+}$ ion channels close. Within this short period of time, the so-called refractory period, the cardiomyocyte can not be depolarized. After the refractory period, the cardiomyocyte is in its resting state again and can also be stimulated to undergoe an action potential again.

The intracellular calcium concentration $\left[\mathrm{Ca}^{2+}\right]_{i}$ is closely related to the contraction of the cardiomyocyte, a mechanism which is referred to on a cellular level as excitation-contraction coupling ${ }^{83}$ or on the macroscopic tissue level as electromechanical coupling. The action potential elicits an increase of the intracellular calcium concentration $\left[\mathrm{Ca}^{2+}\right]_{i}$, as seen in figure 1.6. More specifically, the influx of extracellular $\mathrm{Ca}^{2+}$ ions through the channels leads to a calcium-induced calcium release of intracellular $\mathrm{Ca}^{2+}$ ions by the sarcoplasmatic reticulum and the T-tubules. Released into the sarcoplasm, these calcium ions diffuse rapidly into the myofibrils and catalyze the chemical reactions, which promote sliding of the actin and myosin filaments along one another. ${ }^{68}$ This is the contraction of the cardiomyocyte. Once the intracellular calcium concentration decreases, the contraction ends.

\subsubsection{Myocardium: Functional Syncytium of Cells}

Myocardium is a syncytium of many cardiac cells, in which the cells are interconnected in a way that, when one of the cells becomes excited, the excitation spreads from cell to cell to all of the other cells throughout the myocardium. At their ends, cardiomyocytes are connected through special cell membranes, intercalated discs, which fuse in such a way with each other that ions can diffuse almost freely from cell to cell via gap junctions. ${ }^{68}$ Consequently, action potentials can travel from cell to cell. Cardiac cells are arranged in a latticework of muscle fibers. ${ }^{68}$ Their orientation is orthotropic, meaning that fibers are parallel in one plane. ${ }^{77}$ This orientation rotates in plane-normal direction with each layer throughout the depth of the heart, such that the fiber orientation is rotated by $120^{\circ}$ throughout the wall of the heart. ${ }^{118,222}$ This imposes a special anisotropy onto the conduction of electrical activity, as well as the elastic and contractile properties of myocardium. 


\section{Mechanical Function}

Pulsatile pumping is achieved by the synchronous contraction of all muscle cells of the cardiac muscle. Mechanical contraction of th muscle arises from the entirety of cells exerting contractile forces along their long axis, which coincides with the muscle fiber orientation. The cardiac muscle is striated, meaning that the muscle fibers divide, recombine and spread again. Myocardium is composed of tightly packed cardiomyocytes and fibroblasts, with dense supporting vasculature and a collagenbased extracellular matrix. Fibrillar collagens are the primary structural proteins in the body. ${ }^{77} \mathrm{Me}-$ chanical function of the heart is measured as ejection fraction, which is a measure of the volumetric fraction of blood that is pumped out of the ventricles with each heartbeat.

\subsection{Heart Disease: Cardiac Arrhythmias}

Cardiovascular disease is the leading cause of mortality worldwide, ${ }^{209}$ superceeding the mortality rates of war, traffic accidents or all cancers combined. Cardiac arrhythmias can be associated with almost all forms of heart disease, as they are symptomatic of pathophysiological conditions of the heart. The term 'cardiac arrhythmia' refers to heart rhythm disorders in which the heart rate becomes irregular and typically faster or slower than normal, ${ }^{238}$ which causes a decreased function of the heart. The normal heart beat is a steady, even rhythm, referred to as sinus rhythm, with the sinoatrial nodes and the Purkinje system pacing the heart at rates typically ranging in between 60 and 100 beats per minute in humans. During normal sinus rhythm, the synchronous, orchestrated activation of the cardiac muscle leads to a strong contraction and pulsatile function of the heart. During cardiac arrhythmias, however, this effective pumping becomes reduced or entirely disrupted due to asynchronous, disordered and irregular activations of the cardiac muscle.

Most cardiac arrhythmias are not immediately life-threatening, however, they often present a serious medical condition which requires therapy. Other cardiac arrhythmias, such as ventricular tachycardia or fibrillation, see below, present life-threatening medical emergencies which require immediate intervention. The underlying causes for arrhythmias are diverse, as are its mechanisms and clinical appearances. Symptoms of cardiac arrhythmias typically include chest pain, palpitations or irregular-feeling heartbeats, shortness of breath, dizziness and fainting and may include for critical incidents also loss of consciousness. Cardiac arrhythmias can follow injury or other pathophysiological changes of the heart, which can be attributed to a wide list of origins such as, for instance, myocardial infarctions following a blockage of coronary arteries and decreased oxygen supply during a heart attack, or possibly following infections with an inflammatory infiltration during myocarditis or rheumatic fever, inbalances or depletion of electrolytes due to dehydration, medication or effects associated with genetic predispositions or other diseases that lead to alterations of the cardiac substrate such as for instance cardiac fibrosis or hypertrophy. ${ }^{238}$

\subsubsection{Cardiac Tachyarrhythmias: Tachycardia and Fibrillation}

Cardiac tachyarrhythmias are highly irregular heart rhythm disorders which present very serious and potentially life-threatening medical conditions. The heart rate becomes many times faster than during normal sinus rhythm due to abnormal, chaotic activity in the atria or the ventricles. The activity is often self-sustained and due to its high frequencies able to render the pace-making system ineffective, see following sections, such that if the activity does not end by itself it becomes impossible for the heart to end or override the chaotic activity using its own electrogenic system. This is the highly 


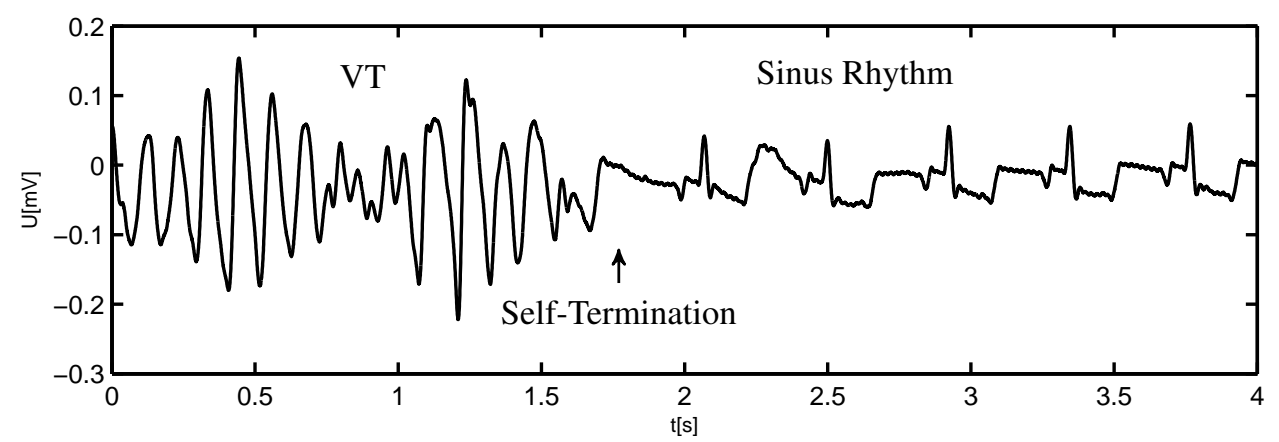

Figure 1.7: Cardiac tachyarrhythmias in the electrocardiogram: spontaneously self-terminating ventricular fibrillation with a transition from fibrillation $(t<0.0 \mathrm{~s})$ to polymorphic tachycardia (VT: $0.0 s \leq t \leq 2.0 \mathrm{~s})$ to normal sinus rhythm $(t>2.0 \mathrm{~s})$ in isolated Langendorff-perfused rabbit heart. Original data obtained in this work, see chapter 6.

life-threatening situation that is ultimately embodied by cardiac tachyarrhythmias, as meanwhile the pumping function of the heart is reduced and the body experiences a lack of oxygen supply without being able to end the condition by itself. The activity arises due to several triggering mechanisms, see section 1.3.3, and may appear but also vanish spontaneously. Because it is difficult to predict the actual circumstances that trigger a particular arrhythmic activity and because tachyarrhtyhmias can also occur in otherwise healthy patients which have not shown any previous predispositions or symptoms, many incidents can not be treated appropriately, leading to numerous cases of sudden cardiac death each year. ${ }^{212}$

Cardiac tachyarrhythmias occur in the myocardial muscle tissue of the upper as well as the lower chambers and are often characterized by their appearance in the electrocardiogram. Accordingly, the frequency and the degree of regularity or irregularity of the electrocardiogram can be used to distinguish atrial tachycardia or fibrillation or ventricular tachycarda or fibrillation respectively.

\section{Tachycardia}

Tachycardia is associated with rapid activity of the heart, many times faster than the regular rapid heart beat. The term 'tachycardia' refers to a range of states of the heart with increased heart rates, many of which are considered benign. ${ }^{238}$ However, tachycardia also refers to potentially dangerous medical conditions, depending on the speed and type of rhythm and whether the arrhythmicity originates in the atria or the ventricles. ${ }^{239,240}$ Tachycardia is typically an unstable rhythm, which can appear spontaneously and also revert to normal sinus rhythm. However, in other cases it can also be persistent. It is associated with increased morbidity and mortality as it can degenerate quickly into more dangerous cardiac tachyarrhythmias, such as atrial or ventricular fibrillation. Tachycardia typically exhibits sinusoidally-shaped mono-chromatic, high-frequency traces in the electrocardiogram, which completely override the normal signature of the sinus rhythm in the case of ventricular tachycardia or modulate the isoelectric baseline in between QRS-complexes, if the arrhythmic activity originates in the atria. ${ }^{123}$ Ventricular tachycardia is distinguished to be either monomorphic or polymorphic, depending on the monochromaticity or degree of irregularity of the frequency components in the electrocardiogram. Polymorphic ventricular tachycardia typically exhibits characteristic undulations of the sinusoidally shaped traces. It is often referred to as 'torsades de pointes', which 
translates to 'twisting of spikes'. ${ }^{239,240}$ Polymorphic ventricular tachycardia is already composed of highly irregular activity, which easily degenerates into even more irregular and complex activity. Ventricular tachycardia causes reduced pumping of the heart.

\section{Atrial Fibrillation}

Atrial fibrillation is the most frequently encountered heart-rhythm disorder in clinical practice. ${ }^{200}$ Atrial fibrillation is associated with increased morbidity and mortality, however, as it originates in the atria it is often not immediately life-threatening because the contribution of the pumping of the atria is not significantly influencing the blood-circulation in the body. However, atrial fibrillation has a tendency to become a chronic condition. As a result, atrial fibrillation is the major cause of stroke, ${ }^{38}$ can result in congestive heart failure and can increase the risk of thrombo-embolism. ${ }^{238}$ Chronic atrial fibrillation is often asymptomic, but can also produce symptoms such as palpitations, fainting, chest pain and other symptoms. ${ }^{238}$

\section{Ventricular Fibrillation}

Ventricular fibrillation is a highly lethal cardiac tachyarrhythmia which leads to death within few minutes if left untreated. Ventricular fibrillation is composed of highly irregular activity in the ventricles that entirely disrupts the coordinated contraction of the cardiac muscle. As the ventricles propel blood through the body, the blood flow becomes cessated, which typically leads to a loss of consciousness and death, if the arrhythmia is not stopped by an electrical shock or other interventional technique, see section 1.4. Ventricular fibrillation can vanish but is more often sustained. In the electrocardiogram, ventricular fibrillation presents with highly irregular traces, which are smaller in amplitude than during ventricular tachycardia. ${ }^{239,240}$

\subsubsection{Complex Wave Patterns underlying Cardiac Arrhythmias}

The billions of cells making up the cardiac muscle together with the anatomical, structural, physiological and functional complexity of the heart present an enormously complicated system whose behavior is very difficult to predict. ${ }^{228}$ Where each individual cell's behavior is determined by the rich dynamics of its numerous ion channels, the vast sum of cells can exhibit highly complex spatialtemporal pattern formation phenomena. These macroscopic phenomena, however, have shown to be very similar to phenomena observed in other biological, chemical and physical systems, see sections 1.1.2, 1.3.3 and 2.1, which were studied over the years by physicists and mathematicians from the perspective of dynamical and nonlinear systems and chaos theory. ${ }^{19,23,27,28}$ For instance, during cardiac arrhythmias one can observe wave phenomena in the heart that exhibit period-doubling bifurcations, ${ }^{166}$ a mechanism that is well-known within the field of nonlinear dynamics and that is typically associated with the emergence of chaotic behavior in deterministic dynamical systems. In attempts to understand cardiac fibrillation, it has ever since been a strategy to relate the behavior of the cardiac muscle with its patterns of activation. Over the years, advances in the field were made by an interdisciplinary community of researchers consisting of cardiologists, electrophysiologists, biologists, biomedical engineers, physicists and mathematicians that were together able to reveal many of the underlying biophysical processes at work during cardiac arrhythmias. Moreover, they provided theoretical explanations as well as experimental evidence for the global action potential wave pattern underlying cardiac tachyarrhythmias. For instance, nowadays, it is possbile to attribute patterns of activation with different types of cardiac arrhythmias, see section 1.3.3. While it has shown to be 
very promising to follow concepts from the field of theoretical physics for explaining cardiac activation patterns, ${ }^{234}$ the understanding of the complicated, highly dynamic pattern underlying cardiac tachyarrhythmias remains incomplete. This section introduces and reviews briefly the historical development that lead to nowaday's understanding of cardiac fibrillation. For a review of the history of the field see also Pandit at al. ${ }^{229}$ and Jalife. ${ }^{200}$

\section{Circulating Electrical Activity and Reentry}

Cardiac fibrillation has attracted the attention of researchers for more than 100 years. Since the early 20th century, researchers started to have the experimental tools at hand that allowed them to investigate the phenomenon of the fibrillating cardiac muscle systematically. ${ }^{200}$ Soon, in 1914, they began to understand that the self-sustained nature of cardiac fibrillation is related to circulating activity of electrical excitation ${ }^{1,2}$ and only shortly thereafter, in 1920, introduced the concept of reentry. ${ }^{3}$ Reentry refers to a prerequisite in the behavior of the electrical activity in order to become self-sustained during cardiac arrhythmias. The terminology itself determines the thinking of the underlying mechanisms of cardiac fibrillation until today. ${ }^{153}$ In section 1.2.2, the behavior of the action potential was introduced. The action potential gives rise to the propagation of nonlinear waves of excitation in cardiac tissue, see also section 1.1.2 and section 2.1 in chapter 2. It starts with a depolarization of the cell's membrane and ends with its repolarization, which is typically followed by a short period, in which further depolarization of the cell is not permitted, see section 2.1. This phase is referred to as the refractory period or excitatory gap. During action potential wave spread in cardiac tissue, the refractory period leads to a directed propagation of the wave. The wave spread is maintained by the diffusion-driven, subsequent electrical activation of neighbouring cells, with each cell experiencing a depolarization of its transmembrane potential that causes also surrounding cells to depolarize. The subsequent activation of neighbouring cells constitutes the action potential wave front. Due to the refractory period, the action potential wave spread is limited to occur along propagation directions, in which the cells were not previously activated. The refractory behavior of the cells leads to a blockage of the propagation of further electrical activity along these directions. Consequently, the action potential wave front is followed by a refractory wave back. Both wave front and back together constitute an action potential wave in cardiac tissue. Subsequent action potential waves are separated by an excitable gap in between their backs and fronts. During normal sinus rhythm of the heart, the refractory wave back prohibits undesired reactivation of the cells after each heart beat. Uncoordinated reactivation of the cells would lead to undesired asynchronous contractile activity of the heart muscle. The entirety of cells within the heart muscle is required to be activated at once to create a strong, orchestrated and synchronous contraction of all cells together. Hence, the refractory period is a mechanism that maintains the controlled extinction of the electrical activity and resets all cells to a state, in which they together become susceptible for a new electrical stimuli supplied by the pace-making system of the heart. The idea of reentry is that the electrical activity is able to circumvent the refractory period or excitable gap and subsequently re-enters the myocard before the pace-making system of the heart is able to reinduce electrical activity. This behavior leads to early, undesired activation of the cardiac muscle and supports, in principle, indefinite, self-sustained activity. The following decades, it was believed that reentry would occur along anatomical circuits or ring-like paths ${ }^{3}$ in the heart. The electrical activity was thought to circulate around the ring-like paths, with the wave length being shorter than the path and an excitable gap separating the wave from its own back. 


\section{Rotors, Multiple Wavelets and Spiral Waves}

The picture of circulating electrical activity and anatomic reentry underlying cardiac arrhythmias was not challenged until the 1950s, when researchers began to seek for alternative explanations for fibrillation, driven by increasing experimental evidence ${ }^{5}$ that either contradicted the concept of circulatory electrical conduction pathways or showed that it was incomplete. The experimental findings, that were particularly related to atrial fibrillation, ${ }^{200}$ suggested that fibrillation was composed of far more irregular activity than circulatory activity alone would be able to produce. Accordingly, Moe ${ }^{9,12}$ postulated in the early 1960s that instead of a single reentrant circle, multiple randomly wandering wavelets were responsible for the highly irregular fibrillatory activity. Also, for decades, the understanding was that the rotating activity would occur due to the involvement of an anatomical obstacle. In the late 1960s and 1970s Krinsky ${ }^{13}$ and Winfree ${ }^{26,27}$ introduced the concept of spiral and scroll waves as a mechanism that leads to rotating activity independently of the existence of obstacles within the cardiac substrate. Spiral and scroll waves are two- and three-dimensional rotating patterns, see section 1.1 and section 2.1.4 in chapter 2, that form dynamically due to the inherent behavior of the substrate that supports the activity. The first experimental observation of spiral waves in the heart was made in 1990 by Davidenko and co-workers, ${ }^{31}$ measuring the epicardial transmembrane potential $V_{m}$ on the epicardial surface using optical fluorescene techniques. Since then, the technique has become the gold-standard in the field and has provided many visualizations of spiraling electrical action potential wave patterns on the surface of the heart, ${ }^{166}$ see also figure 1.8. Nevertheless, the arrhythmic electrical pattern that can be observed on the surface of the heart during ventricular fibrillation can exhibit far more complicated, highly irregular, chaotic patterns, which resemble only intermittently spiral wave patterns, see figure 1.8 and section 6.6.1. It is due to a lack of imaging technology that is able to provide a panoramic, in-depth visualization of the rapidly evolving volumetric arrhythmic electrical wave pattern underneath the surface of the heart, that the understanding of fibrillation has remained incomplete. Today, the electrical wave pattern is described to be turbulent, ${ }^{203}$ insinuating that in addition to a scroll wave structure, there may be other dynamical processes at work underlying fibrillation. ${ }^{234}$

\subsubsection{Clinical Relevance of Chaotic Wave Pattern}

Spiral and scroll wave rotors are nowadays believed ${ }^{48-50,59,65,107,228,229}$ to underlie ventricular fibrillation. Different signatures of the electrocardiogram, which can be associated with different stages and types of heart rhythm disorders, can also be associated with different patterns of the electrical activity. For instance, ventricular tachycardia is associated with one large spiral wave rotor that circulates around a stationary location on the surface of the heart. Polymorphic ventricular tachycardia is associated with one nonstationary spiral wave rotor or possibly a pair of large meandering counterrotating spiral waves. ${ }^{44}$ Lastly, ventricular fibrillation is associated with multiple rotors, often significantly smaller in size than the dimensions of the heart, meandering and performing seldomly more than one full rotation.

The observation of rotating and spiraling electrical activity on the surface of the heart during ventricular fibrillation, ${ }^{40,44}$ together with the observation of rotating scroll waves that lead to spatial-temporal chaos in three-dimensional excitable media, suggests that vortex filament instabilities and interactions, which were found to be responsible for the onset and perpetuation of self-sustaining spatialtemporal disorder in those systems, are also a plausible explanation for the emergence of ventricular fibrillation in the cardiac muscle. Understanding of the basic initiation and trigger mechanisms and 
Chapter 1. Introduction
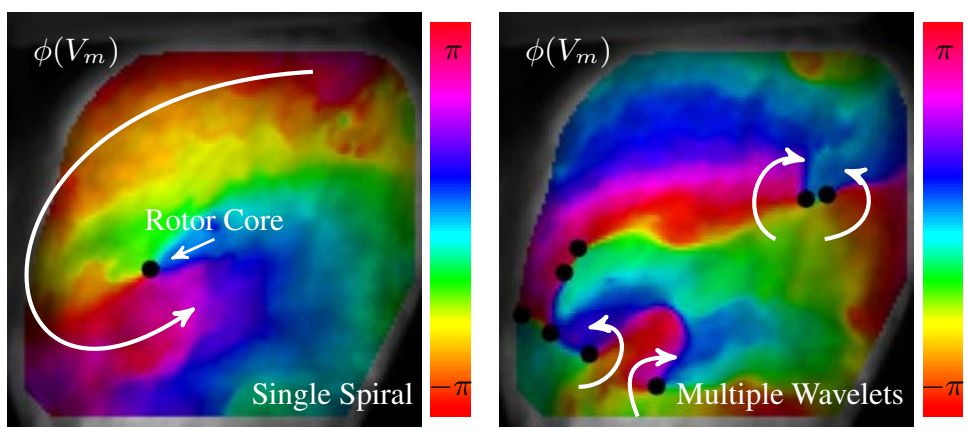

Figure 1.8: Action potential spiral wave patterns on epicardial surface of left ventricular wall of rabbit heart during ventricular fibrillation imaged with fluorescence imaging (optical mapping), see chapter 6 . Large counter-clock-wise rotating spiral during intermittent, more ordered episode (left panel) and highly irregular episode (right panel) with complex spatial-temporal activation pattern with many small rotor-like wavelets due to wave break-up (phase representation $\phi\left(V_{m}\right)$ of transmembrane potential $V_{m}$, data and explanation of colorcode see chapter 6). Rotational core regions indicated by black dots.

dynamic properties of the underlying arrhythmic activity determines the strategies that are developed to intervene cardiac arrhythmias, see following section. Recent low-energy anti-fibrillation pacing techniques ${ }^{177,203}$ assume that scroll waves within the cardiac muscle interact with the heterogeneous cardiac substrate 227 and postulate that it is possible in turn to interact with scroll waves via the heterogeneous substrate using electrical fields. Also the development of catheter ablation strategies for treatment of cardiac arrhythmias ${ }^{220}$ involves the assumption that spiraling electrical activity is driving the arrhythmic activity. Generally, all therapies aiming to intervene or control arrhythmic cardiac activity have in common that they aim to prevent reentry of electrical excitation, that are spiral and scroll waves, see following section.

\section{Triggers Mechanisms of Cardiac Arrhythmia: Heterogeinity and Reentry}

Cardiac arrhythmias are though to be triggered by structural as well as electrophysiological heterogeneities that lead to reentry. The prevailing idea is that alterations of the cardiac substrate lead to anatomical or electrophysiological instabilities that promote wave break-up of the action potential wave pattern. ${ }^{234}$ The break-up then is followed by a consequent perpetuation of disordered scroll wave activity. Alterations, for instance, can be caused by structural remodeling of the cardiac substrate during fibrosis, or spatial dispersion of refractoriness during cardiac alternans. For instance, upon myocardial infarction, dead cardiac cells are removed by macrophages and endothelial cells migrate to the lesion and form granulation tissue, which in turn forms a thick and stiff collageneous scar. ${ }^{119}$ Scar formation reduces the contractility of the heart and constitutes a possible discontinuity in the conducting syncytium, which consequently can lead to wave break-up. This behavior, together with the induced remodeling of the tissue mechanics, can lead to arrhythmia.

\subsection{Intervention and Therapy}

Therapeutic intervention of cardiac arrhythmias is diverse and depends on the individual form of disease. For the acute intervention of cardiac tachyarrhythmias electrical therapies such as cardioversion 
and other defibrillation techniques are used. A reduction of the susceptibility of the heart to arrhythmias is typically aimed by pharmacological therapies. Regenerative therapy of heart tissue is aimed by tissue engineering or cell therapy approaches. Lastly, surgical procedures can be used to stop and help prevent arrhythmia.

\subsubsection{Cardioversion and Defibrillation}

Cardioversion is an electrical therapy used to treat acute ventricular fibrillation by the application of a single high energy electrical shock to defibrillate the heart. ${ }^{180}$ This technique reliably excites all cardiomyocytes at once, which overwrites all self-sustained high-frequency excitation patterns and effectively resets the heart to a state zero in which its own pace-making system can take over. However, due to the high energies used, cardioversion is very painful and can damage the cardiac tissue. ${ }^{182}$ Anti-tachycardia pacing is a more gentle and effective technique in which a series of lowintensity pacing stimuli, delivered directly into the tissue via a single electrode, for instance from an implantable cardioverter-defibrillator (ICD), are used to terminate Tachycardias, lower frequency arrhythmias. ${ }^{180}$ Nevertheless, the technique is less effective for terminating high-frequency arrhythmias like atrial fibrillation.

\subsubsection{New Approaches: Low-Energy Anti-fibrillation Pacing}

Low-energy anti-fibrillation pacing (LEAP) is a recently developed technique, ${ }^{177,203}$ which delivers a short series of low-intensity pacing stimuli at the frequency of anti-tachycardia pacing, but from field electrodes, to terminate cardiac tachyarrhythmias in acute interventional situations. The applied pulsed electrical field recruits sites within the cardiac muscle as so-called virtual electrodes ${ }^{150}$ to emit excitation waves directly into the tissue without implanting physical electrodes. The excitation is thought to be emitted from the hetereogeneous cardiac substrate, from various non-conductive anatomical structures like blood vessels or fatty tissue ${ }^{203,225,227}$ and to interact with core regions of scroll wave rotors, while these rotors pin to the heterogeneities themselves. As a result, the technique is thought to interact with scroll wave rotors directly through the cardiac substrate. The technique has been demonstrated to be highly effective and be able to save large percentages of the required energies to terminate arrhyhmias, promising it to be much less harmful ${ }^{182}$ and painful than cardioversion.

\subsubsection{Ablation Therapy}

Ablation therapy ${ }^{173,174,194}$ is used to selectively create controlled lesions in the myocardium at locations which were found to be sources of arrhythmic activity or which were found to be parts of other abnormal pathways of electrical activity. The resultant scar tissue then blocks abnormal electrical activity. Ablation can be performed using ultrasound, laser light or cryothermy. Usually, the procedure is minimally invasive and can be done via a catheter. Many patients suffering from chronic atrial fibrillation can be cured by catheter-based ablation procedures. ${ }^{221,241}$ Catheter ablation procedures can last up to 6 hours, during which the mapping of the tissue requires most of the time. Presently, the development of techniques that accurately predict the locations of sources or patterns of abnormal electrical activity are a major challenge in the field. The locations are rather estimated from multiple sequential electrode recordings. The latter aspect of this therapeutic approach is of particular interest to this work, see section 1.5 . 


\subsubsection{Other Therapy: Pharmacological Therapy, Cardiac Tissue Engineering and Cell Therapy}

Pharmacological therapy targets molecular processes, which were found to predispose the heart to arrhythmias, using antiarrhythmic drugs. Pharmacological therapy can help prevent heart attack and stroke but can also slow, for instance, the progression of coronary heart disease. ${ }^{238}$ Cardiac tissue engineering and cell therapy aim to compensate the irreversible loss of cardiomyocytes that can be caused by heart disease, by either promoting the formation of new tissue, improving the function of an existing tissue in-vivo, or by generating functional three-dimensional tissue outside the body, in-vitro, before implantation. ${ }^{89,116,135}$ Cardiac myocytes are terminally differentiated cells that can not replicate after injury. ${ }^{88}$ As a consequence, the heart can not compensate cell loss that occurs during myocardial infarction or chronic heart failure. The main goal of myocardial tissue engineering is to improve function of impaired myocardium by integrating cardiac myocytes that were produced outside the body into the native heart architecture to regenerate or even entirely replace damaged heart tissue. Cardiac myocytes have an inherent preference to aggregate and it was shown that isolated cells from immature hearts retain the capacity to reform heart-like tissues under cell culture conditions. ${ }^{7}$ Engineering myocardial tissue uses this property of cardiac cells. The general paradigm is to enclose cardiac cells in an extracellular-matrix-like scaffold or graft material to form a three-dimensional tissue construct. Furthermore, the idea of tissue engineering is based on the possible creation of artificial, functional tissue from stem cells. It is envisioned that the potential use of induced pluripotency stem cells will provide a human autologous source for tissue engineering. ${ }^{184}$

\subsection{Scientific Aim and Structure of this Thesis}

Understanding the complex, self-organized electrical wave pattern underlying life-threatening cardiac arrhythmias remains a major scientific challenge, see section 1.3.2. The development of highresolution fluorescence imaging of electrical activity on the surface of the heart has greatly improved the understanding of cardiac arrhythmias. ${ }^{128,129,153,163}$ Despite this significant progress, fluorescence imaging does not permit the acquisition of information on electrical activity from inside the cardiac muscle, due to the low penetration depths of light in cardiac tissue. It is generally agreed that cardiac arrhythmia is an intrinisically three-dimensional phenomenon. Accordingly, the lack of appropriate three-dimensional imaging techniques imposes a substantial, impeding limitation.

This thesis aims to overcome this limitation of existing cardiac imaging techniques. Using highresolution tomographic ultrasound to capture the mechanical deformation of the heart, this thesis explores novel approaches to visualize complex wave activity inside the cardiac muscle and investigates the relationship between the spatial-temporal dynamics of contractile and electrical activity.

The approach presented in this thesis is based on the assumption that the elasto-mechanical behavior of the deforming cardiac muscle reflects characteristic dynamical properties of the corresponding electrical wave pattern that causes the heart to deform. It is therefore expected that imaging of the mechanical deformations inside the heart's wall during fibrillatory contractile activity would provide insight into the spatial-temporal self-organization during cardiac fibrillation corresponding to the intramural electrical wave patterns. The approach exploits the cardiac excitation-contraction coupling mechanism, which regulates the coupling of electrical and mechanical activity of the heart on the cellular level, and depends on its potential manifestation in closely coupled electromechanical activity 
on the tissue level. It is proposed here that electromechanical activity in the heart is a closely coupled entity that retains a wave-like character with electrical waves causing a wave-like spread of mechanical activation, which subsequently allows the inverse imaging of electrical activity from mechanical deformation of the heart.

The main objective of this thesis is to provide a proof-of-concept of the possibility to perform inverse imaging of electrical activity from mechanical deformation and to demonstrate the feasibility of this approach to visualize transient intramural wave patterns that underlie cardiac tachyarrhythmias.

This thesis combines experimental as well as computational work to investigate the electromechanical activity of the heart during cardiac tachyarrhythmias and explores ways to identify the electrical activity from the structure of the mechanical activity.

The experimental work involves:

- study of ventricular fibrillation in intact, isolated rabbit hearts using high-speed optical fluorescence and ultrasound imaging simultaneously, in order to acquire action potential and calcium transient wave patterns on the surface of the heart, as well as the elasto-mechanical activity on both the surface and inside the heart walls

- design and testing of possible experimental imaging setups and imaging configurations to be able to perform imaging as described above

- establishing that ultrasound imaging provides the desired imaging performance, such as speeds and spatial resolutions, that are necessary to resolve transient fibrillatory activity, as well as ensuring that it provides the capability of producing movie data of deforming cardiac tissue that can be used to extract the time-varying rapidly changing elastic deformation patterns

- analysis of electrical and mechanical wave patterns, comparing the patterns to each other to identify similar features between the two patterns that allow the association of the behavior of the elastic activity with particular behaviors of the electrical pattern

- identification of equipment and imaging technology, which is pertinent to the task of imaging electromechanical wave activity

The computational work involves:

- development of post-processing procedures that are necessary to be able to conduct the measurements, for instance the tracking of the cardiac deformations or the extraction of fluorescence from moving cardiac substrates

- development of a computational model, consisting of a multi-physical description of electrical impulse propagation and accordingly contracting and deforming soft tissue, that allows the investigation of coupled electromechanical wave activity in cardiac muscle tissue in-silico

- development of computational procedures, with which the simulation data can be analyzed and visualized

Here, the in-silico studies aim at establishing fundamental understanding of coupled electromechanical wave activity inside cardiac muscle tissue and, in particular, providing insight into the spatialtemporal patterns that are produced by the activity. Results are to guide the planning of experiments 
with real cardiac tissue preparations, as well as enable interpretation of the measurements.

This thesis is organized as follows: chapters 5 and 6 present the main findings and results. Chapter 5 presents the in-silico studies and chapter 6 presents the experimental results. Generally, results are presented in chapters 4, 5 and 6 , where chapter 4 describes the work of the implementation of the computational model developed and used in this thesis for the in-silico studies. Chapter 2 provides an introduction to the mathematical backgrounds. Chapter 3 contains an extensive literature review of imaging techniques, which were found to be pertinent for this study, but also presents original imaging data of cardiac tissue preparations obtained with different imaging techniques. The last chapter contains a summary of the results, conclusions and recommendations for further study. Each results chapter contains further literature references, description of the used methodolgy as well as a detailed documentation of experimental materials and methods. 


\section{Chapter 2}

\section{Mathematical Modeling of Cardiac Tissue}

Mathematical modeling allows to describe the complex behavior of cardiac tissue qualitatively as well as quantitatively. Computer simulations based on theses descriptions can reproduce and predict various phenomena observed in cardiac tissue on the level of single cells as well as on the level of large populations of cells and the level of the entire organ respectively. Also, mathematical modeling allows to describe different physical processes, such as electrochemical or elasto-mechanical processes. For instance, it is possible to simulate electrical impulse propagation through myocardial muscle tissue or to mimick the elastic deformations of the cardiac muscle. To simulate a beating heart, it thus becomes possible to couple the two physical processes in such ways that the electrical activity triggers contractions of the muscle in a multi-physical description respectively.

This chapter introduces general concepts of mathematical modeling of cardiac tissue and similarly identifies the modeling concepts necessary for the purposes of this study. Next to experimental studies with intact cardiac tissue in-vitro, see chapter 6 , this study aims at investigating coupled electromechanical activity of the heart in silico, to elucidate the fundamental elasto-mechanical behavior of the actively contracting cardiac muscle during ventricular tachycardia and fibrillation, see chapter 5 . In this study, mathematical modeling is used to re-create qualitatively phenomena that can be observed when imaging the heart during cardiac tachyarrythmias, see chapter 6 , using computer simulations based on numerical implementations of these mathematical descriptions, see chapter 4 . The computer simulations enabled the reproduction and interpretation of the experimental findings made in this thesis.

In principle, mathematical and computational modeling of the heart can be done in great detail. For instance, it is possible to simulate the behavior of large populations of cardiac cells, where each individual cell is described in terms of its ion channel dynamics. The dynamics of the entirety of cells then accordingly arises due to the conduction dynamics in the multicellular substrate. However, due to the enormous computational expense resulting from the complexity of these calculations, the situation requires to chose a desired level of detail. Also here, the situation required to chose the appropriate tailor-made computational approach, that was able to reproduce the important studied phenomena, but also proved to be computationally efficient as many of the involved questions revolved around activity in large cardiac tissue volumes. This chapter presents mathematical and computational methods and techniques used in this thesis. 


\subsection{Cardiac Electrophysiology}

The electrophysiology of cardiac muscle tissue is considered to govern the dynamics of the heart, as electrical excitation triggers the contractions of cardiac muscle cells. Mathematical modeling of cardiac electrophysiology has evolved from mathematical descriptions of the electrophysiology of neuronal cells. Neuronal and cardiac cells are both electrically excitable cells and exhibit very similar electrochemical behavior, which is typical for nervous tissue. Both cell types may experience depolarizations of their transmembrane potentials, exhibit action potentials, see section 1.2.2, and connect to each other to form a network that supports electrochemical signal transduction. While neurons form complicated neuronal networks, cardiac cells connect to each other via gap-junctions, to form a continuum or syncytium of cells which supports the propagation of nonlinear waves of electrical excitation. The first mathematical description of the electrical behavior of nerve tissue was studied for electrical impulse propagation in the giant squid axon by Hodgkin and Huxley ${ }^{6}$ in 1952. The model has had a significant impact on the field and was the founding for various other descriptions of nonlinear phenomena in biology including the development of the first mathematical electrophysiological model of cardiac tissue proposed by Noble et al. ${ }^{11}$ in 1962.

Mathematical modeling of cardiac electrophysiology generally consists of a description of the electrochemical processes occuring in each individual cardiac cell and a description of the cellular interconnections, which support signal transduction through the tissue and allow to study the behavior and interactions of large populations of cells.

\subsubsection{Single Cell and Ion Channel Dynamics}

The electrical behavior of the single cardiac muscle cell determines the behavior of the entire tissue and is determined in turn by the interplay of its numerous ion channels that regulate the electrochemical processes of the cell. The idea behind mathematical modeling of the cell's electrical behavior is to capture the dynamics of the transmembrane potential $V_{m}$. In general physical terms, the cell membrane is considered to act as a capacitor and its ion channels as a resistor and regulator for electrical charges and currents flowing through the membrane. ${ }^{45,76,114}$ The membrane's electrical capacity can be loaded by the accumulation of ionic charges $Q$ on the membrane and the transmembrane potential, which is the resultant potential difference $V_{m}=v_{i}-v_{e}$ between the intracellular and extracellular potentials, builds up accordingly and proportionally to the membrane capacitance $C_{m}$ :

$$
\frac{d Q}{d t}=C_{m} \frac{d V_{m}}{d t}
$$

The change of charge $\dot{Q}$ on the membrane is caused by the flow of ionic currents through the ion channels $\dot{Q}=I$. Kirchhoff's law implies that the sum of all ionic currents flowing through the membrane is zero:

$$
\begin{aligned}
0 & =\frac{d Q}{d t}+I_{N a^{+}}+I_{K^{+}}+I_{C a^{2+}}+I_{C l^{-}}+\ldots \\
\frac{d Q}{d t} & =-I_{N a^{+}}-I_{K^{+}}-I_{C a^{2+}}-I_{C l^{-}}-\ldots
\end{aligned}
$$

where the right hand side is the total ionic transmembrane current $I_{m}$ of the different ion species. The electrical behavior of the cell in the absence of flow of any other transmembrane current $I$ is 
therefore fully described by the ordinary differential equation:

$$
0=C_{m} \frac{d V_{m}}{d t}+I_{m}
$$

A net flow of transmembrane current $I$, as it would occur for instance after the application of external stimulation, is then described as follows: ${ }^{60}$

$$
I=C_{m} \frac{d V_{m}}{d t}+I_{m}
$$

This formulation allows the detailed description of the electrical behavior of the cell by taking into account each contribution of a single ion species and its respective ion channel to the net flow of charges and the corresponding resultant transmembrane voltage. Each ionic current $I_{i o n}=\left\{I_{N a^{+}}, I_{K^{+}}, \ldots\right\}$ is of the form:

$$
I_{i o n}=g_{i o n}\left(V_{m}-V_{i o n}\right)
$$

where $g_{i o n}$ is the specific conductance of the respective ion channel and $V_{i o n}$ is the corresponding reversal or Nernst potential, see also equation 1.2.1. In particular, the ion-specific conductances $g_{i o n}$ cause the nonlinearity in the electrical behavior of the cell, which leads subsequently to the formation of an action potential. The conductance of an ion channel $g_{i o n}(\vec{h}, t)$ is a time-varying parameter which depends on gating variables $\vec{h}$, which in turn describe transitions in the behavior of the ion channel. ${ }^{45,76}$ For instance, the transmembrane potential may affect voltage-gated ion channels whereas mechanical stress may affect stretch-activated ion channels. Hence, precise and physiologically detailed modeling requires detailed knowledge about the molecular functioning of ion channels. Indeed, many involved quantities of the underlying molecular biophysical processes can be determined experimentally using electrophysiological measurement techniques such as, for instance, voltage- and patch-clamp techniques. ${ }^{188}$ Equations 2.1.5 - 2.1.6 are referred to as Hodgkin-Huxleytype modeling ${ }^{6}$ of cardiac electrophysiology. Many cardiac electrophysiology models are based on similar approaches including large numbers of ordinary or partial differential equations describing ion channels and gating variables. ${ }^{76,114,171}$ On the one hand, this quantitative, biophysical modeling approach has shown to be very successful in describing accurately the rich electrical behavior of cardiac muscle cells. On the other hand, it requires a vast amount of information and is computationally expensive, which is in many cases inappropriate or impossible when aiming to simulate the entire cardiac muscle, which consists of billions of cardiac cells. Its complexity makes this approach also inapplicable for the purposes of this study.

\subsubsection{Cell Syncytium and Spreading Waves of Electrical Excitation}

To describe the dynamics of the cardiac cell embedded in a continuum or syncytium of cells, that allows the spread of electrical excitation from cell to cell, one needs to specify the currents resulting from the intercellular coupling with neighboring cells. This can usually be approximated using a diffusive term as in the following equation:

$$
\begin{aligned}
\nabla \cdot(\mathbf{D} \nabla) V & =C_{m} \frac{\partial V}{\partial t}+I_{m} \\
\nabla_{\mathbf{D}}^{2} V & =C_{m} \frac{\partial V}{\partial t}+I_{m}
\end{aligned}
$$


where $\nabla_{\mathbf{D}}^{2}=\nabla \cdot(\mathbf{D} \nabla)$ is a term for the diffusive process, with $\mathbf{D}$ being a tensor of intercellular conductivities, and $\nabla$ being the gradient operator. The external current $I$ presented in equation 2.1.5 can here be understood to be given by current leaking through gap-junctions and other diffusive connections into the cell. The integration over a continuum of cells then constitutes a description for the propagation of nonlinear waves of excitation in cardiac tissue.

\subsubsection{Reaction-Diffusion Systems}

Reaction-diffusion systems are mathematical models which describe spatial-temporal conversion and transport processes of substances. ${ }^{175}$ Typically, reaction-diffusion systems are used to describe local changes of concentrations of compounds in chemical reactions. However, examples of reactiondiffusion systems can be found in chemistry, biology, geology, physics and ecology. ${ }^{35,42,175}$ Reactiondiffusion systems are described by a set of partial differential equations:

$$
\frac{\partial \mathbf{y}}{\partial t}=\nabla^{2} \mathbf{y}+\mathbf{F}(\mathbf{y})
$$

where $\mathbf{y}$ is a vector of dynamic state variables describing the local state of the reaction-diffusion medium and the two terms on the right side of the equation correspond to the diffusive transport $\nabla^{2} \mathbf{y}$ and to the local reaction $\mathbf{F}(\mathbf{y})$, which is typically a nonlinear term. The above formulation implies that the dynamic behavior occurs in a continuum or a spatially extended system consisting of many constituents and that dynamical activity arises from diffusion-like local transport processes among constituents together with local reactions in each constituent. It is easy to note that equation 2.1.9 is very similar to the Hodgkin-Huxley equation 2.1.8. Indeed, reaction-diffusion systems provide a general mathematical framework for the description of cardiac electrophysiology. ${ }^{190}$ However, they are a very generic mathematical framework for the description of non-equilibirum dynamic systems ${ }^{42}$ in general and can produce a wide array of spatial-temporal patterns, with cardiac tissue being only one of the most prominent representatives.

\subsubsection{Excitable Media}

Excitable media are a sub-class of reaction-diffusion systems with particular behavior of the local kinetics $\mathbf{F}(\mathbf{y})$ in equation 2.1.9 that facilitates the emergence of nonlinear waves of excitation ${ }^{175}$ and enables modeling of cardiac electrophysiology. ${ }^{190}$ Excitable media retain three characteristic and outstanding features, see also section 1.1.1: they possess a resting state, the property of excitability with an excitation threshold and a refractory period. These features or properties emerge from the local kinetics $\mathbf{F}(\mathbf{y})$, which are inherent in each of the constituents of an excitable medium. In the following, the dynamic state of the constituents is described to exist in a so-called dynamic phase space. ${ }^{80}$ The idea of the phase space is that a phase state of a dynamical system can be described by a vector with $N$ dynamic state variables as entries in a $N$-dimensional state vector space. Accordingly, a dynamical evolution of a state corresponds to a trajectory through the phase space. The default dynamic state of each of the constitutents of an excitable medium is the resting state, as the resting state is a so-called attractor in phase space. Excitation occurs only if a local finite perturbation threshold is exceeded by a perturbation, which elevates the state away from the attractor above a separatrix and induces an excursion of the dynamic state through the dynamic phase space given by the equations describing the local kinetics. The excursion occurs only for a finite time as the dynamic state tours through the dynamic phase space on a trajectory that returns to the initial resting state and attractor. However, in the meantime the dynamic state passes through its excited state followed by a refractory 
period at the end of the excursion during which further excitatory activity is inhibited. In general mathematical terms, the trajectory of the dynamic state is determined by the shape of the underlying manifold that arises from the equations defined in $\mathbf{F}(\mathbf{y})$. In particular, the above described dynamic behavior requires a nonlinearity in the local kinetics term $\mathbf{F}(\mathbf{y})$. Also, the underlying manifold of excitable media promotes quasi-periodic behavior of the dynamics. For each perturbation exceeding the threshold, the dynamic state experiences an excursion from resting over the excited to the refractory state and back to the resting state and this cycle can be repeated as long as the system contains energy that supports the excursions. This dynamic behavior enables the propagation of nonlinear waves of excitation in a continuous spatial domain. As neighboring constituents of an excitable medium interact with each other by diffusion-like local transport processes, the excitation can spread from constituent to constituent and trigger new excursions leading to spreading nonlinear waves of excitation. The diffusion process is maintained by the diffusive term $\nabla^{2} \mathbf{y}$ in equation 2.1.9.

In terms of cardiac electrophysiology, the excursion through phase space corresponds to an action potential and the nonlinear waves of excitation correspond to action potential wave propagation in the myocardial muscle. The resting state corresponds to the resting potential of the cardiac cell and the perturbation and perturbation threshold correspond to a depolarizing electrical stimuli and the cell's reversal potential respectively. The diffusion corresponds to the intercellular connectvity that allows exchange of ionic electrical charges.

In fact, excitable media retain many features of cardiac electrophysiology and are frequently used to describe and simulate the electrical behavior of the heart. Typically, excitable media, and similarly reaction-diffusion systems, are formulated using sets of few coupled partial differential equations, which are designed in ways that the dynamical behavior of the global system resembles that of cardiac electrophysiology, which truly emerges from the complex interplay and individual behavior of numerous ionic currents on the sub-cellular level. The Hodgkin-Huxley equations presented in section 2.1.1, which take into account the electrophysiological complexity in great detail, retain similarly many properties which assert to excitable media. In essence, the Hodgkin-Huxley model is the description of an excitable medium. ${ }^{8}$ However, conceptually, the approach of describing the electrophysiological activity using an ionic-based model, that assembles the global behavior synthetically by adding up the actual individual contributions of ionic currents, is very different from a description that starts with the macroscopic behavior and specifies the underlying mathematical equations appropriately to produce the globally arising dynamics phenomenologically, see section 2.1.5. The latter modeling approach stems from dynamical and non-equilibirum systems theory and employs reaction-diffusion systems and excitable media under the assumption that it is possible to extract the important and generic parts of the underlying dynamics of a complex system, at the same time preserving its key characteristics and qualitative behavior. ${ }^{8,190,228}$

More examples of excitable media in biological, chemical and physical systems are given in the introduction in section 1.1.1.

\section{Spiral and Scroll Waves}

Typical phenomena observed in excitbale media are the formation of spiral waves in two and scroll waves in three spatial dimensions respectively. ${ }^{27,28,41,52}$ A general introduction to the phenomenon was given in section 1.1.2 in the introductory chapter. Spiral waves emerge from so-called reentrant excitatory activity in spatially extended excitable systems. Reentrance refers to a break-up of the 

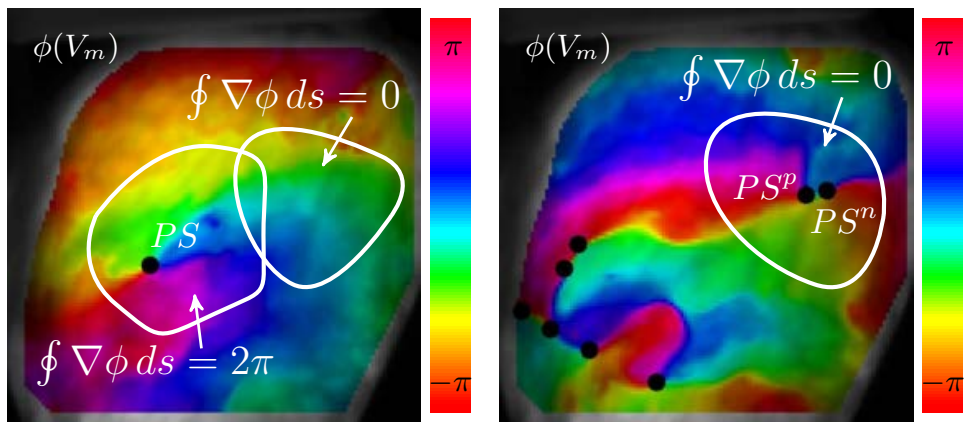

Figure 2.1: Phase singularities (PS) on epicardial surface of left ventricular wall of rabbit heart during ventricular fibrillation imaged with fluorescence imaging (optical mapping), see chapter 6. Path integrals of the spatial gradient of the phase $\phi$ within the phase plane yield $2 \pi$ if they enclose a phase singular point and 0 otherwise, see left panel. However, if the path integral encloses two phase singularities with opposite topological charge the integral also vanishes, see right panel. The topological charge of a phase singularity indicates the rotational sense of the underlying rotor pattern.

directed propagation of nonlinear waves of excitation with the possibility of waves to spread through their own broken-up wave front in the opposite propagation direction. Reentrance may occur due to several reasons and depends on system properties, such as the substrate properties but also intrinsic properties of the dynamic system itself.

\section{Phase Singularities and Scroll Vortex Wave Filaments}

Spiral and scroll wave activity can be characterized by phase singularities ${ }^{59,125}$ which mark the rotational core regions of the rotational patterns. Phase singularities correspond to a phase singular point in two dimensions and a line of phase singularity in three dimensions respectively. Phase singular points indicate the spiral wave tip and lines of phase singularity correspond to vortex filaments which indicate the rotating center of a scroll wave. Generally, a phase angle of oscillation $\phi$ can be attributed to the periodic activity of the medium. Within the phase representation or phase portrait of the activity, the activity produces lines or surfaces of equal phase, that is, for instance, the wave front of the nonlinear waves of excitation corresponds to one line or surface of equal phase in two or three dimensions respectively. Accordingly, at the tip of a spiral, in two dimensions, all lines of equal phase merge, see figure 2.1. If one is to compute the integral of the gradient of the phase within the phase plane along a closed path around one particular site, one obtains vanishing integrals when the path does not enclose a tip of a spiral and a non-vanishing integral in the case that the path encloses the tip of the spiral and only the tip of this one spiral. More precisely, the sum of the gradient of the phase around a given site yields along the circular path:

$$
2 \pi(n-p)=-\oint_{s} \nabla \phi d s
$$

where $n$ and $p$ are the number of phase singularities enclosed with negative and positive topological charge respectively and $\nabla \phi$ is the spatial gradient of the phase along the path $s$. Hence, if the circular path encloses two phase singularities with opposite topological charge the integral yields 0 . In particular, the integral also yields 0 if the path does not surround any phase singular point with $n=$ 0 and $p=0$, as can be seen in the left panel in figure 2.1. In computer simulations, where the dynamic 
state variables are available at all times, the phase state of activation can easily be computed. ${ }^{59,125}$ For instance, for models using two dynamic state variables, see next section, the dynamic state occurs on circular trajetories in a two-dimensional phase state space. However, in the analysis of experimental data the phase state of oscillation of the dynamic state is often not immediately known from the measurement and needs to be reconstructed. This can be achieved either by identifying discrete events in time, for instance the upstroke of the action potential, and interpolating the phase linearly in between two of these events or by embedding the measured signal into a higher dimensional dynamic phase space. For instance, delay embedding or the hilbert transformation can be used to reconstruct a two-dimensional dynamic phase space from a measured time-series, from which in turn the phase angle can be deduced. ${ }^{59}$

\subsubsection{Phenomenological Models of Cardiac Electrophysiology}

Phenomenological models of cardiac electrophysiology aim to capture the fundamental, qualitative electrical behavior of the myocardial muscle. They are very often based on descriptions using sets of coupled partial differential equations, following the concept of excitable media and reaction-diffusion systems, see section 2.1.4. The idea behind the phenomenological modeling approach is to reduce the complexity of the description of cardiac electrophysiology to be able to understand and control its behavior using concepts from nonlinear dynamics. At the same time, this approach provides a modeling framework that allows to study the behavior of large populations of cells, as the computational complexity gets reduced. This, in turn, allows to study self-organizing, spatial-temporal patterns of electrical activity in the cardiac muscle, which typically consists of billions of cells. This section introduces three popular phenomenological models used for modeling of cardiac electrophysiology. Two of the models were used in this thesis, implemented in numerical computational models, see chapter 5 .

\section{The FitzHugh-Nagumo Model}

The FitzHugh-Nagumo model ${ }^{8,10}$ is a two-variable phenomenological model which is typically used to describe electrical impulse propagation in nerve fiber and myocardial muscle tissue. The model consists of a set of two coupled nonlinear partial differential equations with two dynamic variables:

$$
\begin{aligned}
& \frac{\partial u}{\partial t}=\nabla^{2} u+u-\frac{1}{a} u^{3}-v+I \\
& \frac{\partial v}{\partial t}=\varepsilon(u+b-c v)
\end{aligned}
$$

where $u$ and $v$ are dimensionless, normalized dynamic state variables or excitation and recovery variables respectively, $\varepsilon$ is a parameter determining the separation of timescales of the two dynamic variables, $I$ is the magntiude of an externally applied stimulus and $a, b$ and $c$ are constant parameters determining the general behavior of the system. Depending on the choice of parameters, the FitzHugh-Nagumo model can exhibit three operating regimes in which it exhibits either excitable, oscillatory or bistable dynamics. 
The FitzHugh-Nagumo model was designed to explain the basic properties of excitability as exhibited by the more complex Hodgkin-Huxley model, isolating conceptually the essentially mathematical properties of excitation and propagation from the electrochemical properties of sodium and potassium ion flow. ${ }^{134}$ It summarizes the many different Hodgkin-Huxley equations in only two equations, therefore reducing the complexity of the Hodgkin-Huxley model to a model that is analytically tractable, can be examined easily in a two-dimensional phase space, has minimal computational requirements and allows to study excitable media qualitatively. Nevertheless, the FitzHugh-Nagumo model is a generalized version of the Van-der-Pol oscillator and also describes the nonlinear behavior of relaxation oscillators. Even though the FitzHugh-Nagumo model was not used directly in this thesis, it needs to be mentioned here as it is one the most prominent qualitative models for electrical impulse propagation in nerve tissue and is the original model for many other modeling approaches.

\section{The Aliev-Panfilov Model}

The Aliev-Panfilov mode ${ }^{51}$ is a two-variable FitzHugh-Nagumo type phenomenological model, describing cardiac electrophysiology. It consists of a set of two coupled nonlinear partial differential equations with two dynamic variables:

$$
\begin{aligned}
\frac{\partial u}{\partial t} & =\nabla^{2} u-k u(u-a)(u-1)-u v \\
\frac{\partial v}{\partial t} & =\varepsilon(u)(k u-v)
\end{aligned}
$$

where $u$ and $v$ are dimensionless, normalized excitation and recovery variables respectively. The parameter $a$ defines the threshold of activation. The parameter $k$ controls the magnitude of the excitation. The parameter $\epsilon(u)$ sets the time-scale of the recovery process with $\varepsilon(u)=1$ for $u<0.05$ and $\epsilon(u)=0.1$ for $u \geq 0.05$. A different, more flexible formulation of the Aliev-Panfilov model is given by:

$$
\begin{aligned}
\frac{\partial u}{\partial t} & =\nabla^{2} u-k u(u-a)(u-1)-u v \\
\frac{\partial v}{\partial t} & =\varepsilon(u)(-k u(u-a-1)-v)
\end{aligned}
$$

Here, the parameter $\varepsilon$ is not case dependent but rather depends on the dynamic variables $u$ and $v$ as follows: $\varepsilon(u, v)=\frac{\varepsilon_{0}+\mu_{1} v}{u+\mu_{2}}$ with $\varepsilon_{0}=0.002, k=8$ and $\mu_{1}, \mu_{2}$ being parameters. The Aliev-Panfilov model is a derivative and extension of the FitzHugh-Nagumo model, ${ }^{8}{ }^{8} 10$ with modifications that were intended to adapt the model to the specific behavior of cardiac electrophysiology, such as action potential duration and restitution. A very similar model to the Aliev-Panfilov model is the Barkley model. ${ }^{34}$

\section{The Fenton-Karma Model}

The Fenton-Karma model ${ }^{58}$ is a phenomenological model consisting of a set of three coupled nonlinear partial differential equations describing cardiac electrophysiology. The model aims to depict 
cardiac electrophysiology more realistically and also more physiologically-based than the aforementioned phenomenological models. Parts of its mathematical formulations aim to relate directly to electrophysiological processes of the cardiac cell. The model introduces three transmembrane currents, a fast inward current $I_{f i}$, a slow outward current $I_{s o}$ and a slow inward current $I_{s i}$, which roughly correspond to the sodium current, the potassium current and the calcium current of the cardiac cell, respectively, see also section 1.2.2. The Fenton-Karma model is given by a set of three coupled nonlinear partial differential equations:

$$
\begin{aligned}
\frac{\partial u}{\partial t} & =\nabla^{2} u-I_{f i}(u, v)-I_{s o}(u)-I_{s i}(u, w) \\
\frac{\partial v}{\partial t} & =\theta\left(u_{c}-u\right)(1-v)\left(\frac{\theta\left(u-u_{v}\right)}{\tau_{v 1}^{-}}+\frac{\theta\left(u_{v}-u\right)}{\tau_{v 2}^{-}}\right)-\theta\left(u-u_{c}\right) \frac{v}{\tau_{v}^{+}} \\
\frac{\partial w}{\partial t} & =\theta\left(u_{c}-u\right) \frac{1-w}{\tau_{w}^{-}}-\theta\left(u-u_{c}\right) \frac{w}{\tau_{w}^{+}}
\end{aligned}
$$

where the dynamic state is described by three dynamic state variables with $u$ being the excitatory variable and $v$ and $w$ being refractory variables respectively. The variables can be given either in dimensionless, normalized units, then the resting state is $(u, v, w)^{t}=(0,1,1)^{t}$, or in dimensions where the dimension of the first variable $u$ corresponds to the transmembrane voltage $V_{m}$ in millivolts $m V$. The first equation contains the expressions for the three different ionic transmembrane currents:

$$
\begin{aligned}
I_{f i}(u, v) & =-\frac{v}{\tau_{d}} \theta\left(u-u_{c}\right)(1-u)\left(u-u_{c}\right) \\
I_{s o}(u) & =\frac{u}{\tau_{0}} \theta\left(u_{c}-u\right)+\frac{1}{\tau_{r}} \theta\left(u-u_{c}\right) \\
I_{s i}(u, w) & =-\frac{w}{2 \tau_{s i}}\left(1+\tanh \left(k\left(u-u_{c}^{s i}\right)\right)\right)
\end{aligned}
$$

where $\theta$ is the Heaviside-function and all parameters $\tau_{r}, \tau_{s i}, \tau_{0}, \tau_{w}^{+}, \tau_{v 1}^{-}, \tau_{v 2}^{-}, \tau_{w}^{+}, \tau_{w}^{-}, \tau_{d}, u_{c}, u_{v}$, $u_{c}^{s i}, k$ in equations 2.1.17 through 2.1.20 relate to time-scales or threshold-values of the system. The three equations for the ionic currents do not depict the complexity of the ionic currents of a cardiac cell but rather summarize the most dominat channels and ionic net current flows. The FentonKarma model is able to reproduce also detailed behavior of cardiac electrophysiology, for instance action potential shapes with steep upstrokes and restitution behavior. The increase in computation time is significant when switching from using the two-variable Aliev-Panfilov model to the threevariable Fenton-Karma model. However, the Fenton-Karma model is one of the least computationally expensive models for realistic modeling of cardiac electrophysiology.

\subsubsection{Restitution}

Restitution is an important and characteristic feature of cardiac electrophysiology, referring to the behavior of the action potential duration and the refractory period in dependence of the activation 
frequency of the tissue. Formally, restitution describes the relationship between the duration of the action potential and the length of the diastolic interval. Generally, the action potential duration shortens with shortening diastolic interval, that is, the action potential duration decreases with higher activation frequency, for instance. However, typically, for higher activation frequencies, one can observe that the decrease of the action potential duration does not decrease further at the same rate at which the diastolic interval is decreasing. Restitution behavior is often related to cardiac arrhythmias as short diastolic intervals inroduce a vulnerable phase, a phase in which waves can be blocked by the repolarization phase of the previous wave and wave break becomes possible, see also section ??. Out of the phenomenological models presented above, see section 2.1.5, only the Fenton-Karma model is able to reproduce the phenomenon of restitution in cardiac tissue properly. The Aliev-Panfilov model exhibits restitution, however, to an extent that barely realistic.

\subsubsection{Calcium Dynamics}

Calcium plays a major role in cardiac electrophysiology. It determines the repolarizing behavior of the action potential as well as its duration and, more importantly, triggers and fuels the contraction of cardiomyocytes, ${ }^{83}$ see also sections 1.2 and 2.3. Calcium is also one promoter for the formation of cardiac alternans. ${ }^{230}$ Modeling of calcium dynamics is therefore an important field in modeling cardiac electrophysiology. ${ }^{161}$ However, in this work, detailed intracellular calcium dynamics were neglected as it was desired to study the simplest possible electromechanical activity to establish a relationship between spatial-temporal coupled electromechanical patterns assuming homogenous active tension development and direct conversion of 'excitation' into myofibril contraction. Hence, it was assumed that calcium would immediately follow the course of the action potential.

\subsection{Cardiac Tissue Elasto-Mechanics}

Cardiac muscle cells, cardiomyocytes, shorten in length by up to $20 \%$ when contracting. ${ }^{77}$ The ventricular muscle consists of billions of cardiac muscle cells, which lead together to a strong deformation of the muscle when contracting. Deformations of myocardial muscle tissue are typically considered to be large or finite deformations. Therefore, a description of the continuum mechanical kinematics needs to be based on finite strain and deformation theory. ${ }^{14}$ This section introduces mathematical concepts and terminology used to describe soft tissue and in particular, the elasto-mechanical behavior of the actively contracting cardiac muscle, see also section 1.2.3.

\section{Continuum Mechanics and Kinematics}

In continuum mechanics, kinematics refers to the mathematical description of the deformation and motion of a piece of material. It includes tensor mathematics, which depicts the complicated spatial relationships of material behavior during a deformation. The continuum hypothesis assumes that characteristic length scales of the microstructure are much smaller than characteristic length scales of the overall problem. ${ }^{77}$ Also, finite strain and deformation theory typically assumes nonlinear behavior of the involved equations. In this work, mathematical modeling of the elastic behavior of cardiac tissue aims to describe macroscopic cardiac tissue, such as the ventricular wall. During an affine deformation properties of the deformation on macroscopic length scales also translate uniformly to microscopic length scales. 


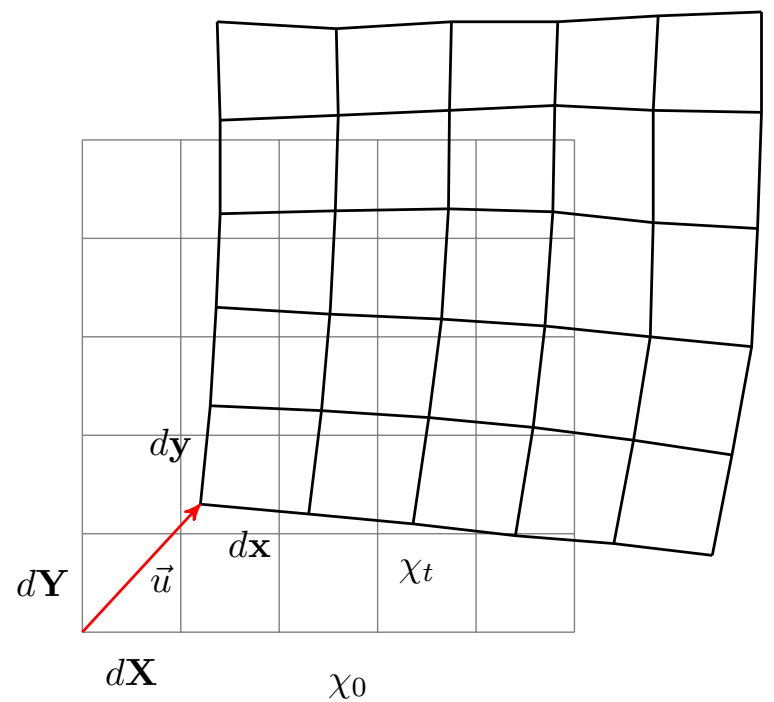

Figure 2.2: Configurations of a material continuum body: material particle at location $\mathbf{X}=\left\{X_{M}\right\}$ with $M=1,2,3$ in reference configuration $\chi_{0}$ as rectangular regular lattice, and material particle at location $\mathbf{x}=\left\{x_{j}\right\}$ with $j=1,2,3$ in deformed configuration $\chi_{t}$, displacement $u_{j}$ with $j=1,2,3$

\subsubsection{Configurations of a Continuum Body}

In finite strain and deformation theory, ${ }^{14}$ the kinematic description of a continuous medium is based on the concept of a configuration, in which a continuum body is situated, see also figure 2.2. A continuum body consists of a number of material particles $P^{i}$ with $i=1, \ldots, N$, which continuously fill a volume $\Omega \in \mathbb{R}^{3}$. A configuration of a continuum body is the continuous mapping of all its particles into three-dimensional space $\mathbb{R}^{3}$.

\section{Frame of Reference}

Assume that $\mathbf{x}=\left\{x_{j}\right\}$ with $j=1,2,3$ describes the deformed or current position of a material particle, that occupied the location $\mathbf{X}=\left\{X_{M}\right\}$ with $M=1,2,3$ in the reference or undeformed configuration in rectangular Cartesian coordinates. ${ }^{144}$ At time $t$ the particle located at the initial location $\mathbf{X}$ occupies the place $\mathbf{x}(\mathbf{X}, t)$ and it subsequently at time $t+\delta t$ occupies the place $\mathbf{x}(\mathbf{X}, t+\delta t)$. A reference or undeformed configuration $\chi_{0}$ is a configuration in which the continuum body is situated typically in an undeformed, stress-free state. The continuum body will return to the reference configuration if no load is applied and the deformation is elastic. A deformed or current configuration $\chi_{t}$ subsequently describes the continuum body in a deformed state. Note that we use lower case indices for quantities associated with the deformed continuum body, and upper case indices for quantities associated with the undeformed continuum body. Furthermore, we use indices notation as well as tensor notation. The coordinates of the particle's position in the reference configuration $\mathbf{X}$ are referred to as the material coordinates of the particle, while $\mathbf{x}$ are its spatial coordinates. The reference configuration is also referred to as Lagrangian configuration. The deformed configuration is also referred to as Eulerian configuration. A transformation of a body from the reference configuration to the deformed or current configuration is a deformation. In the following, we consider deformations as mappings from the Lagrangian into the Eulerian space. 


\section{Displacement}

The displacement vector $\mathbf{u}$ describes the translation or displacement of a material particle $P_{\mathbf{X}}$, situated at location $X$ in the reference configuration, to the same material particle $P_{\mathbf{x}}$, situated at a location $\mathrm{x}$ in the deformed configuration. If the coordinate systems of the reference and the deformed configurations coincide, the location of a material particle in the current location $\mathrm{x}$ can be expressed by its reference location $\mathbf{X}$ and an according displacement $\mathbf{u}$ :

$$
\mathbf{x}=\mathbf{X}+\mathbf{u}
$$

The displacement vector $\mathbf{u}=\mathbf{x}-\mathbf{X}$ joins the positions of the material points $P_{\mathbf{X}}$ and $P_{\mathbf{x}}$ from Lagrangian and Eulerian space, see figure 2.2. The configuration of a continuum body is changed by the displacements $\mathbf{u}^{i}$ of its material particles $P^{i}$.

\subsubsection{Deformation and Strain}

A deformation has occurred if there is a relative displacement $d \mathbf{u}=d \mathbf{x}-d \mathbf{X}$ between adjacent material particles after a global displacement of the continuum body has occured. Adjacent particles of a continuum body can be described by infinitesimal material line elements that retain a specific position and orientation inside the continuum body, see figure 2.2. These vectors are denoted as $d \mathbf{X}$ in the reference configuration and $d \mathbf{x}$ in the deformed configuration. A relationship between the material orientation vector in the reference and the deformed configuration is given by:

$$
d x_{j}=\frac{\partial x_{j}}{\partial X_{M}} d X_{M}
$$

written in indices notation.

\section{Deformation Gradient Tensor}

We define the mapping itself, that is the partial differentiation of $x_{j}$ with respect to $X_{M}$, as the gradient deformation tensor $\mathbf{F}$ :

$$
\begin{aligned}
F_{j M} & =\frac{\partial x_{j}}{\partial X_{M}} \\
& =\left(\begin{array}{lll}
\frac{\partial x_{1}}{\partial X_{1}} & \frac{\partial x_{2}}{\partial X_{1}} & \frac{\partial x_{3}}{\partial X_{1}} \\
\frac{\partial x_{1}}{\partial X_{2}} & \frac{\partial x_{2}}{\partial X_{2}} & \frac{\partial x_{3}}{X_{2}} \\
\frac{\partial x_{1}}{\partial X_{3}} & \frac{\partial x_{2}}{\partial X_{3}} & \frac{\partial x_{3}}{\partial X_{3}}
\end{array}\right)
\end{aligned}
$$

The gradient deformation tensor $\mathbf{F}$ is a mapping between the reference configuration and the deformed configuration, see figure 2.2. It deforms an infinitesimal material line element $d \mathbf{X}$ from the reference configuration into an infinitesimal material line element $d \mathbf{x}$ in the current configuration:

$$
d \mathbf{x}=\mathbf{F} d \mathbf{X}
$$

The gradient deformation tensor $\mathbf{F}$ is a second order tensor because it has two independent indices $j=1,2,3$ and $M=1,2,3$. In particular, $\mathbf{F}$ is not symmetric. Since myocardial tissue is incompressible with:

$$
\operatorname{det}(\mathbf{F})=0
$$


the mapping $\mathbf{F}$ is reversible and invertible with $|\mathbf{F}| \neq 0$. Tissue deformation can be described via tensor fields with each tensor corresponding to one material particle. The deformation gradient tensor can be determined from the displacements $\mathbf{u}$, which transform the reference configuration into the deformed configuration. Partial differentiation of equation 2.2.1 with respect to the reference coordinates $X_{M}$ yields:

$$
\begin{aligned}
F_{M}^{j} & =\frac{\partial x_{j}}{\partial X_{M}} \\
& =\frac{\partial X_{M}}{\partial X_{M}}+\frac{\partial u_{j}}{\partial X_{M}} \\
& =\delta_{N M}+\frac{\partial u_{j}}{\partial X_{M}} \\
& =\mathbb{I}+\nabla_{X} \mathbf{u}
\end{aligned}
$$

with $\delta_{N M}$ being the non-vanishing derivatives and II being the identity matrix. Consequently, the deformation gradient tensor $\mathbf{F}$ can be expressed in terms of the displacement gradient tensor $\nabla_{X} \mathbf{u}$. The displacement gradient tensor $\nabla_{X} \mathbf{u}$ is accessible from the displacements $\mathbf{u}$ in a displacement vector field $\mathbf{D}$, derived from registered motion of experimental data.

\section{Strain and Deformation Measures}

Closely related to the concept of deformation is the concept of strain. In the simplest possible formulation, strain is defined as change in length per unit of initial length: ${ }^{14}$

$$
\varepsilon=\frac{L-L}{L}=\frac{d x^{\prime}-d x}{d x}=\frac{d u}{d x}
$$

It is consequently a dimensionless quantity or normalized measure of deformation comparing lengths of material elements to their reference lengths. In general terms of finite deformation theory, strain is defined as relative displacement of material particles in the continuum body with respect to each other. An important quantity relating strain and deformation is the right Cauchy-Green deformation tensor $\mathbf{C}$, which is defined as:

$$
\begin{aligned}
\mathbf{C} & =\mathbf{F}^{T} \mathbf{F} \\
& =\frac{\partial x_{j}}{\partial X_{N}} \frac{\partial x_{j}}{\partial X_{M}}
\end{aligned}
$$

The right Cauchy-Green deformation tensor is a derivative of the gradient deformation tensor $\mathbf{F}$. It transfers a squared infinitesimal length $d \mathbf{X}^{2}$ in the Lagrangian configuration into the corresponding squared infinitesimal length $d \mathbf{x}^{2}$ in the Eulerian configuration, i.e. $d \mathbf{x}^{2}=d \mathbf{X} \cdot \mathbf{C} d \mathbf{X}$. It is a quantity describing the square of local change in distances due to deformation. Note that $\mathbf{C}$ is symmetric by definition. The quantity $d \mathbf{x}^{2}-d \mathbf{X}^{2}$ in between two neighboring material particles is used as a measure of deformation, which occurs in the vicinity of the particles between the reference and the deformed configuration,. ${ }^{132}$ If the difference is zero, a rigid displacement has occurred. For instance, figure 2.2 shows a deformed continuum body with configuration $\chi_{t}$ at time $t$. The right 
half of the continuum body has translated, while the left half rests in its reference configuration. However, relative displacements among adjacent particles are minimal in the translated part of the continuum body. A rigid deformation has occurred. In between both sections, we find a region in which the local relative displacements are large. The strain is localized in this region. A deformation can be composed of strain and rigid-body motion. The difference of the squared infinitesimal lengths $d \mathbf{x}^{2}-d \mathbf{X}^{2}$ of adjacent particles with respect to their respective configurations can be expressed by:

$$
\begin{aligned}
d \mathbf{x}^{2}-d \mathbf{X}^{2} & =d \mathbf{X C} d \mathbf{X}-d \mathbf{X} \amalg d \mathbf{X} \\
& =d \mathbf{X}(\mathbf{C}-\mathbb{I}) d \mathbf{X} \\
& =2 d \mathbf{X E} d \mathbf{X}
\end{aligned}
$$

which results in the Green-Lagrangian strain tensor E:

$$
\begin{aligned}
\mathbf{E} & =\frac{1}{2}(\mathbf{C}-\mathbb{I}) \\
& =\frac{1}{2}\left(\frac{\partial x_{j}}{\partial X_{N}} \frac{\partial x_{j}}{\partial X_{M}}-\delta_{N M}\right) \\
& =\frac{1}{2}\left(\nabla_{X} \mathbf{u}+\left(\nabla_{X} \mathbf{u}\right)^{T}+\nabla_{X} \mathbf{u}\left(\nabla_{X} \mathbf{u}\right)^{T}\right)
\end{aligned}
$$

where $\mathbb{I}$ and $\delta_{N M}$ are the identity matrices respectively and $\nabla_{X} \mathbf{u}$ is the displacement gradient tensor. The Green-Lagrangian strain tensor is a measure of how much $\mathbf{C}$ differs from $\mathbf{I} .^{132}$ It is a local measure for the strain that an infinitesimal material line element between adjacent material particles experiences. The Green-Lagrangian strain tensor's components can be defined solely in terms of material coordinates. The strain tensor describes the material independent change of the geometry of the object. Note that $\mathbf{E}$ is symmetric by definition.

A complete description of the deformation of a three-dimensional continuum body is given by a threedimensional tensor field, with second order tensors as entries for each spatial location and material prism or volume element of the continuum body.

\section{Tensor Invariants and Principal Strains as Measures of Deformation}

The rich information provided by deformation tensors is in many situations difficult to interpret and process conceptually or inconvenient when aiming to visualize the deformation. To be able to express a deformation in simple terms, ideally, a reduction of the tensor data to scalar-valued data becomes necessary. This can be achieved by exploiting the properties of symmetric tensors as, for instance, the Right-Cauchy-Green deformation tensor $\mathbf{C}$ and the Green-Lagrangian deformation tensor $\mathbf{E}$ are symmetric tensors. Tensors are abstract mathematical constructs which describe physical quantities independently of a coordinate system. ${ }^{139}$ Scalars, vectors and square matrices are representations of zero order, first order and second order tensors respectively in a specific coordinate system. Socalled tensor invariants are scalar-valued representations of a tensor, which can be associated with 
specific properties of the tensor independently of the matrix representation of the tensor in a specific coordinate system. Hence, they are invariant under coordinate-transformation. As an example, the magnitude of a vector is invariant and independent of the coordinate system that spans the vector in its respective vector space. Invariants of the Right-Cauchy-Green deformation tensor $\mathbf{C}$ and the Green-Lagrangian deformation tensor $\mathbf{E}$ are often used for the formulations of strain energy density functions, which describe the constitutive behaviour of a material. In this work, tensor invariants and other mathematical properties of the symmetric deformation tensors $\mathbf{C}$ and $\mathbf{E}$ are exploited to depict quantitative features of the deformations of contracting cardiac tissue. The three fundamental tensor invariants of the symmetric Green-Lagrangian deformation tensor $\mathbf{E}$, for instance, read:

$$
\begin{aligned}
I_{1}(\mathbf{E}) & =\operatorname{tr}(\mathbf{E}) \\
& =E_{11}+E_{22}+E_{33} \\
I_{2}(\mathbf{E}) & =\frac{1}{2}\left(\operatorname{tr}\left(\mathbf{E}^{2}\right)-\operatorname{tr}(\mathbf{E})^{2}\right) \\
& =E_{11} E_{22}+E_{22} E_{33}+E_{1} E_{33}-E_{12}^{2}-E_{23}^{2}-E_{13}^{2} \\
& =E_{1} E_{2}+E_{2} A_{3}+E_{1} E_{3} \\
I_{3}(\mathbf{E}) & =\operatorname{det}(\mathbf{E}) \\
& =E_{1} E_{2} E_{3}
\end{aligned}
$$

where $E_{i, j}$ with $i, j=1,2,3$ are the components and $E_{1}, E_{2}, E_{3}$ are the eigenvalues of the deformation tensor $\mathbf{E}$. The first invariant $I_{1}(\mathbf{E})$, the trace of the tensor, is a measure of squared length of a material prism element described by the tensor. That is, the trace of the Green-Lagrangian deformation tensor being smaller zero, $\operatorname{tr}(\mathbf{E})<0$, relates to a compressile deformation, whereas $\operatorname{tr}(\mathbf{E})>0$ relates to a tensile deformation of the material prism element. The third invariant relates to volume changes of the material prism element. As already implied above, tensor invariants of symmetric, positive definite tensors are known to be related to the coefficients of the characteristic polynomial of the tensor:

$$
p(\lambda)=\operatorname{det}(\mathbf{E}-\lambda \mathbb{I})
$$

where $\lambda$ is the polynomials indeterminate and $\mathbb{I}$ is the identity matrix. In other words, the tensor invariants are related to the eigenvalues of the tensor. With $\mathbf{E}$ being a symmetric matrix with $n \times n$ real-valued entries, this matrix has $n$ eigenvalues $\lambda_{i}$ and $n$ orthogonal eigenvectors. The eigenvalue problem states as follows:

$$
\mathbf{E v}_{i}=\lambda_{i} \mathbf{v}_{i}
$$

where $\mathbf{v}_{i}$ are $n$ mutually perpendicular eigenvectors of the tensor $\mathbf{E}$. The eigenvalues $\lambda_{i}$ of the strain tensor are referred to as principal strains or normal strains of the deformation. They express pure dilation or compression along the principal axes of the deformation. The principal or normal axes are given by the eigenvectors $\mathbf{v}_{i}$ that span a principal coordinate system in which shearing strains vanish:

$$
\mathbf{E}=\left(\begin{array}{ccc}
E_{11} & 0 & 0 \\
0 & E_{22} & 0 \\
0 & 0 & E_{33}
\end{array}\right)=\left(\begin{array}{ccc}
\lambda_{1}^{2} & 0 & 0 \\
0 & \lambda_{2}^{2} & 0 \\
0 & 0 & \lambda_{3}^{2}
\end{array}\right)
$$


In the so-called eigen-frame representation the off-diagonal components $E_{12}=E_{21}=E_{31}=0$ are the vanishing tangential or shearing strain components and $E_{11}, E_{22}, E_{33}$ are the principal stretches or squared eigenvalues of the tensor respectively $\lambda_{1}=E_{1}=\sqrt{E_{11}}, \lambda_{2}=E_{2}=\sqrt{E_{22}}, \lambda_{3}=$ $E_{3}=\sqrt{E_{33}}$. The eigenvalues of the Green-Lagrangian deformation tensor can be computed from its characteristic polynomial as follows:

$$
p(\lambda)=\operatorname{det}(\mathbf{E}-\lambda \mathbb{I})=\operatorname{det}\left(\begin{array}{ccc}
E_{11}-\lambda_{1} & E_{12} & E_{13} \\
E_{12} & E_{22}-\lambda_{2} & E_{23} \\
E_{13} & E_{23} & E_{33}-\lambda_{3}
\end{array}\right) \stackrel{!}{=} 0
$$

where expanding the polynomial yields:

$$
\begin{aligned}
p(\lambda)= & E_{11} E_{22} E_{33}-\lambda_{1} \lambda_{2} \lambda_{3}-\lambda_{1} E_{22} E_{33}-\lambda_{2} E_{11} E_{33}-\lambda_{3} E_{11} E_{22} \\
& +\lambda_{1} \lambda_{2} E_{33}+\lambda_{1} \lambda_{3} E_{22}+\lambda_{2} \lambda_{3} E_{11}+\lambda_{1} E_{23}^{2}+\lambda_{2} E_{13}^{2}+\lambda_{3} E_{12}^{2} \\
& -E_{12}^{2} E_{33}-E_{11} E_{23}^{2}+2 E_{12} E_{13} E_{23}-E_{13}^{2} E_{22}
\end{aligned}
$$

Solutions for the roots of the polynomial or eigenvalues $\lambda_{1}, \lambda_{2}, \lambda_{3}$ of the tensor can be obtained numerically but also in closed-form equations analytically. ${ }^{14}$ Principal strains were used frequently in this work to depict deformation patterns qualitatively, see chapter 5.

\subsubsection{Elastic Anisotropy of Myocardial Muscle Tissue}

Ventricular deformation mechanics are very complicated due to the highly anisotropic, hierarchical organization of the myocardial muscle. Deformations of the heart consist of longitudinal, circumferential and radial deformation and twist. This is due to the orthotropic, chirally rotating muscle fiber anisotropy and helical global organization of muscle fiber, muscle fiber sheet and muscle fiber sheet-normal orientations. ${ }^{77}$ However, locally, in one specific sub-volume of the ventricular wall, the elastic anisotropy can be considered to be linearly transverse isotropic, as all fibers are approximately aligned uniformly along one direction within one bundle of fibers. Transversely isotropic materials retain physical properties which are symmetric about one axis, the axis being normal to a plane of isotropy. Tensor invariants are, respectively, invariant under rotation $\mathbf{f} \rightarrow \mathbf{f} \Theta$ around the axis $\mathbf{f}$, where $\mathbf{f}$ is the preferred direction in the transversely isotropic material and $\Theta$ expresses the rotation. Accordingly, a tensor invariant applicable for linearly or transversely isotropic materials is given as follows:

$$
I_{4}(\mathbf{E})=\mathbf{f} \cdot \mathbf{E f}
$$

\subsubsection{Stress and Constitutive Laws}

Stress $\sigma$ is a quantity describing the average force $F$ per unit area of an imaginary internal surface $A$ within a deformable continuum body. Internal forces act between material particles in the continuum body undergoing deformation. These internal forces are distributed continuously within the volume of the continuum body. They may lead to a deformation or they may be induced by the deformation. 
The dimension of stress is pressure. The Cauchy stress tensor $\sigma$ defines stress at any point in a continuum body:

$$
\sigma=\left(\begin{array}{lll}
\sigma_{11} & \sigma_{12} & \sigma_{13} \\
\sigma_{21} & \sigma_{22} & \sigma_{23} \\
\sigma_{31} & \sigma_{32} & \sigma_{33}
\end{array}\right)
$$

The Cauchy stress tensor is a symmetric second order tensor expressing stress with respect to the deformed configuration. Its components can be displayed with respect to the coordinate system, for instance the stresses $\sigma_{11}, \sigma_{22}, \sigma_{33}$ are the perpendicular normal stresses of the planes defined by the normal vectors $e_{x}, e_{y}$ and $e_{z}$ respectively. The stresses acting tangent to the planes are called shear stresses. The Cauchy stress tensor describes stress in a continuum body experiencing small deformations. In contrast to the Cauchy stress tensor, the second Piola-Kirchhoff stress tensor expresses stress relative to the reference configuration $\chi_{0}$, allowing the description of stress in a continuum body experiencing finite deformations. Therefore, it employs the gradient deformation tensor $\mathbf{F}$. The second Piola-Kirchhoff stress tensor $\mathbf{T}$ is a symmetric, second order tensor derived from the Cauchy stress tensor $\sigma$ as follows:

$$
\begin{aligned}
\mathbf{T} & =|\mathbf{F}| \mathbf{F}^{-1} \sigma\left(\mathbf{F}^{-1}\right)^{T} \\
& =|\mathbf{F}| \frac{\partial X_{M}}{\partial x_{j}} \sigma \frac{\partial X_{N}}{\partial x_{j}} \\
& =T_{M N}
\end{aligned}
$$

The second Piola-Kirchhoff stress tensor $\mathbf{T}$ expresses the force per unit undeformed area, acting on an infinitesimal element of surface $d \mathbf{A}$ in the reference conguration $\chi_{0}$. Its components can be defined solely in terms of material coordinates, just as for the Green-Lagrangian strain tensor E. To represent material behaviour independent of rigid-body motion, it is convenient to define the state of mechanical stress and strain of the tissue in terms of the second Piola-Kirchhoff tensor $\mathbf{T}$ and the Green-Lagrangian strain tensor $\mathbf{E}$ respectively. The fundamental equation that governs finite deformation elasticity arises from the conservation of linear momentum following Newton's laws of motion. ${ }^{14,106}$ For static equilibrium in the absence of body forces, the governing equations expressed in terms of second Piola-Kirchhoff stress components reduce to:

$$
\frac{\partial}{\partial X_{M}}\left(T_{M N} F_{M}^{j}\right)=0
$$

with the second Piola-Kirchhoff stress tensor $T^{M N}$ in indices notation.

The elasticity of a continuum body relates its material properties with the deformation. The constitutive behaviour of a material, that is the relationship between stresses and strains, is expressed by elastic constitutive equations. These equations are typically non-linear for materials undergoing finite elastic deformations. Cardiac tissue is generally considered to be a hyperelastic material, which is 
a material for which the stress-strain relationship derives from a strain energy density function. The following strain energy density function $\mathbf{W}$ was proposed by ${ }^{57}$ for myocardial tissue: ${ }^{132}$

$$
\mathbf{W}=\sum_{i=1}^{3} \frac{k_{i} \mathbf{E}_{i i}^{2}}{\left(a_{i i}-\left|\mathbf{E}_{i i}\right|\right)^{\beta_{i}}}
$$

where $k_{i}, a_{i}$ and $\beta_{i}$ are parameters describing anisotropy and inhomogeneity of the myocardium in the fiber, sheet, and sheet normal direction and $\mathbf{E}_{i i}$ are the diagonal components of the GreenLagrangian strain tensor. Strain energy density functions $\mathbf{W}$ depend on the components of the right Cauchy-Green deformation tensor or the Green-Lagrange strain tensor. The differentiation of the energy density function $\mathbf{W}$ with respect to the Green-Lagrangian strain tensor $\mathbf{E}$ results in the second Piola-Kirchhoff stress tensor $\mathbf{T}$ :

$$
\mathbf{T}=\frac{\partial \mathbf{W}}{\partial \mathbf{E}}
$$

The stress $\mathbf{T}$ in the material can be obtained by taking the derivative of the strain energy density function $\mathbf{W}$ with respect to the strain $\mathbf{E}$. This is a fundamental relationship which allows the analytical formulation of the elastic behaviour of a continuum. The dynamical state of a continuum body is consequently fully defined when we know its strain energy function $\mathbf{W}$ and can derive for each particle $P^{(i)}$ its tensors $\mathbf{F}, \mathbf{C}, \mathbf{E}$ and $\mathbf{T}$, consequently obtaining various tensor fields for the tissue configuration $\chi$. Together with the stress equilibirum, equation (2.2.18), we obtain a fully analytical framework to describe an elastic material.

\subsubsection{Computational Modeling of Elasticity}

Soft tissue elasticity is commonly simulated employing either the finite element method, ${ }^{106}$ discrete elastic systems like particle or mass-spring damper systems ${ }^{67,207}$ or other deformable models employed in computer graphics, ${ }^{55,120}$ but also viscoelastic modeling is employed. ${ }^{113}$

\section{Finite Element Method}

The finite element method is a numerical technique to find approximate solutions to partial differential equations or integral equations. Finite element methods employ the discretization of a continuous domain into a set of discrete sub-domains, usually called elements. The discretization into elements is an approximation of the real continuous problem. The discretization usually allows the transformation of a system of partial differential equations describing the continuum mechanical problem into a system of ordinary differential equations, which still describe the problem in good approximation and in their entirety. The numerical implementation of the finite element formulation is a common technique in computer simulations to study tissue elasticity. Solutions from finite element simulations are more accurate compared to other techniques used to model elasticity. ${ }^{133}$ However, the numerical treatment of the finite element formulation is very technical and computationally expensive. Finite element methods were not used in this work. 


\section{Discrete Particle or Mass-Spring Systems}

Soft-tissue elasticity can be modeled using discrete particle systems or, more specifically, massspring elasticity models. ${ }^{55,90,120}$ Mass-spring systems are particle systems with a constrained topology. Neighboring particles are connected with springs or other forcing terms, which introduce attractive and repelling forces into the particle system, to form a lattice or similarly ordered structure. As a result, the system exhibits an elastic response to external loads and converges to its reference configuration once the external load is removed. Achieving physically realistic deformation in realtime, mass-spring models are widely used to simulate biological tissues and were also reported to be used to model cardiac tissue. ${ }^{105,133,201,233}$ Mass-spring models are dynamic models, which allow waves and vibrations. They can easily run in real-time and have accordingly often been chosen when performance was important. They are of great interest to many medical or surgery simulation ${ }^{105}$ and visualization applications and were also, for instance, widely used in real-time cloth or hair simulations. However, the precision with which they simulate the constitutive behaviour of an elastic material is less accurate than when using the finite element methods and depends on the correct identification of spring parameters. ${ }^{147}$

The concept of mass-spring models is to introduce particles and springs to model elasticity. A volume of tissue $\Omega$ is defined as a number of particles $P^{(i)}$ distributed at locations $x_{i} \in \mathbb{R}^{3}$, where $x_{i} \in \Omega$. The particles are volumeless point-masses and exhibit inertia like a physical particle. Between the particles, there are nominal distances which are defined by the initial particle distribution in the tissue volume $\Omega$ in the reference configuration $\chi_{0}$. The springs connect each particle $P^{(i)}$ with its neighboring particles $P^{(j)}$ with $j=1, \ldots, N$. Consequently, a network of nodes and edges is constructed, with nodes being interchangeable with physical particles and edges with springs. The nominal distance defines a resting distance between two particles, which is the resting length $l_{i j}$ of the spring. The particle's motion in 3-dimensional space is governed by the following system of ordinary second order differential equations:

$$
m_{i} \ddot{\mathbf{x}}_{i}=-c \dot{\mathbf{x}}_{i}+\sum_{j} \mathbf{f}_{i j}(\mathbf{x})
$$

where $\dot{\mathbf{x}}$ and $\ddot{\mathbf{x}}$ are the velocity and acceleration of a particle $P^{(i)}$ and $m_{i}$ is its mass. The system is damped by a damping constant $c$. Typically, mass-spring systems are overly damped beyond a realistic amount to increase stability of the system. ${ }^{105}$ External forces are assumed to be zero and are not included in the equation. The term $\sum \mathbf{f}_{i j}$ is a superposition of all internal forces affecting the particle. Each particle $P^{(i)}$ interacts with $j$ neighboring particles. Consequently, the forces are the attracting and repelling forces of the springs. Each force $\mathbf{f}_{i j}$ can be derived as follows:

$$
\mathbf{f}_{i j}=k_{i j}\left(l_{i j}-\left\|\mathbf{x}_{i}-\mathbf{x}_{j}\right\|\right) \frac{\mathbf{x}_{i}-\mathbf{x}_{j}}{\left\|\mathbf{x}_{i}-\mathbf{x}_{j}\right\|}
$$

where $\mathbf{x}_{i}$ is the location of the considered particle $P^{(i)}$ and $\mathbf{x}_{j}$ is the location of the neighboring particle $P^{(j)}$ in three-dimensional space, $k_{i j}$ is the stiffness of the spring and $l_{i j}$ is the rest length of the spring. The spring's stiffness can be linear following Hooke's law with $f=-k x$ or piecewise linear, modeling nonlinear behavior, as follows:

$$
k_{i j}=\left\{\begin{array}{lll}
k_{i j}^{1} & \text { for } & l_{i j}-\left\|\mathbf{x}_{i}-\mathbf{x}_{j}\right\| \leq e_{i j} \\
k_{i j}^{2} & \text { for } & l_{i j}-\left\|\mathbf{x}_{i}-\mathbf{x}_{j}\right\|>e_{i j}
\end{array}\right.
$$


where $k_{i j}^{1}$ and $k_{i j}^{2}$ are two different stiffness constants and $e_{i j}$ is a certain length of the spring serving as a threshold. Mass-spring systems find the minimal energy configuration as they approximate the volume as a finite lattice structure with simplified node-to-node connectivities. ${ }^{105}$ Consequently, equilibirium is computed at the node points. This contrasts with the idea of the finite element method, where the equilibrium is computed in a continuous domain.

\subsection{Electromechanical Coupling}

Cardiac tissue is an actively contracting elastic medium. The classical definition of forces appearing in a continuum body distinguishes external forces acting on a body and internal forces acting in between two parts of the body. ${ }^{14}$ In the classical sense, internal forces describe the elastic response of tissue to tension. In actively contracting tissue, this concept needs to be extended. Therefore, the second Piola-Kirchhoff stress tensor $\mathbf{T}$ can be defined as a summation of passive stress $\mathbf{T}_{p}$, which occurs due to the elastic response of the tissue, as well as active stress $\mathbf{T}_{a}$, which is induced by the contraction of the cardiomyocytes: ${ }^{106}$

$$
\mathbf{T}=\mathbf{T}^{p}+\mathbf{T}^{a}
$$

The active stress arises due to the calcium-induced shortening of muscle fiber bundles. The intracellular calcium-release which fuels the contraction is triggered by the action potential. Phenomenological modeling approaches ${ }^{106,130}$ of this behavior relate the active stress build-up directly to the electrical activation. For instance, in the seminal work by Nash \& Panfilov ${ }^{106}$ a third dynamic variable $T_{a}$ was introduced in adddition the two dynamic variables $u$ and $v$ for the electrophysiological Aliev-Panfilov model, ${ }^{51}$ see also section 2.1.5, to couple the active stress development directly to the excitatory dynamics, providing a straight-forward phenomenologcial description of excitationcontraction coupling:

$$
\begin{aligned}
\frac{\partial u}{\partial t} & =\nabla^{2} u-k u(u-a)(u-1)-u v-I_{s} \\
\frac{\partial v}{\partial t} & =\varepsilon(u)(k u-v) \\
\frac{\partial T_{a}}{\partial t} & =\varepsilon(u)\left(k_{T} u-T_{a}\right)
\end{aligned}
$$

Here, $k_{T}$ is a parameter describing the rate of tension development and $I_{s}(\mathbf{C}, u)$ is a term describing stretch-activated channel currents. The excitation variable $u$ triggers the dynamics of the active stress $T_{a}$. The partial differential equation including the active stress $T_{a}$ features similar dynamics to the equation for the recovery variable $v$. See section 2.1 for a description of the other parameters. Equation 2.3.3 together with equation 2.3.1 is the simplest description for cardiac excitation-contraction coupling.

\subsubsection{Forward and Backward Electromechanical Coupling}

The general paradigm in mathematical modeling of the coupled electrical and mechanical behavior of cardiac tissue ${ }^{106,130}$ is to include the interplay of both entities in a decription of the electrical 
reaction-diffusion system, compare also with equation 2.1.8:

$$
\nabla \cdot\left(\mathbf{D}(\mathbf{C}) \nabla V_{m}\right)=C_{m}(\mathbf{C}) \frac{\partial V_{m}}{\partial t}+I_{m}(\mathbf{C})
$$

Here $\mathbf{C}$ is the right Cauchy-Green deformation tensor. As in equation 2.1.8, $\mathbf{D}$ is a tensor of conductivities, $V_{m}$ is the transmembrane potential, $I_{m}$ is the total ionic transmembrane current and $C_{m}$ is the capacitance of the cell membrane. The right Cauchy-Green deformation tensor $\mathbf{C}$ appears in three terms, which together constitute electromechanical coupling in both the forward direction, that is the conversion of electrical activity into mechanical activity, and the backward direction, that is the feedback of the mechanical activity onto the electrical activity. The first term on the left side of equation 2.3.4 models to the effect of the deformation onto the diffusion properties of the electrical activity, the first term on the right describes membrane capacitance changes due to mechanical deformation of the cell. Both terms correspond to the backwards direction of the coupling. The last term in equation 2.3.4 corresponds to the ionic effects of the deformation. Here, both forward and backward directions of the coupling are included, as the description for the ionic currents may include the calcium activity, but also for instance stretch-activated channels $I_{s}(\mathbf{C}, u)$ of the cells, that may elicit electrical activity due to tensile mechanical deformation. Forward and backward electromechanical coupling together support electromechanical feedback. ${ }^{144}$ These latter effects were not considered in this thesis.

\subsubsection{Elastic Anisotropic Active Stress Development and Elasticity}

The stress equilibrium within an actively contracting soft tissue is given by extending equation (2.2.18) to include the two active and passive stress terms described above:

$$
\frac{\partial}{\partial X_{N}}\left(T_{M N} F_{j M}\right)=0
$$

with the 2. Piola-Kirchoff stress tensor $T_{M N}$ being composed of passive and active stresses:

$$
\begin{aligned}
T_{M N} & =T_{M N}^{p}(\mathbf{C})+T_{M N}^{a}(\mathbf{C}, u) \\
& =\frac{1}{2}\left(\frac{\partial W}{\partial E_{M N}}+\frac{\partial W}{\partial E_{N M}}\right)+T^{a} C_{M N}^{-1}
\end{aligned}
$$

where the Green-Lagrangian strain tensor $\mathbf{E}(\mathbf{C})$ depends on the right Cauchy-Green deformation tensor $\mathbf{C}$ and the active stress tensor $\mathbf{T}^{a}$ depends on $\mathbf{C}$ as well as on the excitation variable $u$. In particular, in case of isotropic tension development, the active stress tensor $\mathbf{T}_{a}$ reduces to a scalar value $T_{a}$ which is subsequently coupled via the inverse Cauchy-Green deformation tensor $C_{M N}^{-1}$ into the stress tensor. 


\subsubsection{Computational Modeling of Electromechanically Coupled Wave Activity}

The Nash-Panfilov model ${ }^{106}$ presented in equations 2.3.1-2.3.3 is a phenomenological FitzHughNagumo type model describing electromechanical coupling in cardiac tissue qualitatively using reaction -diffusion partial differential equations and constitutive elastic material equations, together with a continuous description of the kinematics. Its continuum mechanical description is typically implemented and solved via the finite element method. ${ }^{106,144,181}$ In the original model, the electrophysiological description is implemented by employing the finite differences method. The Nash-Panfilov model is referred to as a reaction-diffusion mechanics system. ${ }^{144,207}$ With the inclusion of the stretchactivated channel current $I_{s}$, it allows to simulate mechano-electrical feedback. Here, electromechanical feedback was neglected. Compared to the vast number of electrophysiological models, there are only few reported multi-physical models that allow simulating coupled mechano-electrical activity in cardiac tissue. To date, Nash-Panfilov-type models are most commonly used in the field and several extensions or modifications of the model have been published. ${ }^{144,156,167,170,181,192}$ Mass-spring models were reported to be used in combinations with electrophysiological models to simulate actively contracting cardiac tissue. ${ }^{132,164,207,208,223,233}$ Due to their numerical efficiency, the latter approach was also followed in this thesis. 
Chapter 3. Imaging of Electromechanical Wave Activity in the Heart

\section{Chapter 3}

\section{Imaging of Electromechanical Wave Activity in the Heart}

There is a wide array of techniques used to image the heart. Imaging applications range from standard, high-throughput diagnostic imaging in the day-to-day clinical setting to highly specialized and customized imaging of the hearts metabolism in cardiac research. The physical principles underlying these numerous imaging techniques are very diverse and may range from optical over electrical and electrophysiologial techniques to techniques employing microfluidics or subatomic nuclear interactions of radiation with tissue. Also, imaging may depend on computational post-processing or inverse imaging techniques, without which the measurement would not be possible.

This chapter presents imaging technology that either provides the possibility to be used or has the potential of being used for imaging electromechanical wave activity and discusses and evaluates its respective performance and applicability. Likewise, this chapter introduces the methods used in this work, see sections 3.1 and 3.2. Also, original imaging data that was acquired with some of the imaging technology is presented.

To measure electromechanical wave activity, it becomes necessary to capture two physically distinct processes: the electrophysiological activity as well as the mechanical deformation and contractility of cardiac tissue. Moreover, it becomes necessary to be able to capture the rapidly evolving activity that occurs throughout the heart, on its surface and within the cardiac muscle, ideally in a panoramic fashion to be able to study electrical and mechanical activity patterns. The upstroke of the action potential occurs within few milliseconds, see section 1.2, and propagation speeds of action potentials in cardiac tissue range in the order of $1.0 \mathrm{~m} / \mathrm{s}$ during normal sinus activity and $0.1-0.5 \mathrm{~m} / \mathrm{s}$ during abnormal, arrhythmic activity. Ventricular fibrillation typically exhibits dominant frequencies of about $10-20 \mathrm{~Hz}$ and electrical patterns observed on the surface of the heart exhibit accordingly similar frequencies. In rabbit hearts, spiral wave rotors observed on the epicardial surface typically exhibit rotational periods of about $70-140 \mathrm{~ms}$ during ventricular tachycardia or fibrillation. The mechanical onset of contraction occurs on similar time-scales as the onset of the action potential, however, figure 1.6 depicts that it does not occur as steep and rapid as the action potential. The size of a rabbit heart is in the order of $1.5 \mathrm{~cm}^{3}$, of a human in the order of $10 \mathrm{~cm}^{3}$. The thickness of the ventricular wall is in a rabbit heart in the order of $1-6 \mathrm{~mm}$ and in humans in the order of $1-3 \mathrm{~cm}$. On the other hand, aside from functional imaging, it is desired to visualize the structure of the heart. The complicated anatomic structure of the heart is ideally displayed using three-dimensional imaging 
Table 3.1: Comparison of imaging modalities for imaging of electrical and mechanical activity in the heart: imaging mode, acquisition speeds and penetration depths

\begin{tabular}{|c|c|c|c|}
\hline modalitiy & $\begin{array}{l}\text { penetration depth } \\
{[\mathrm{mm} / \mathrm{cm}]}\end{array}$ & imaging mode & $\begin{array}{l}\text { acquisition speed [fps / vps] } \\
\text { sampling rate }\end{array}$ \\
\hline$\overline{\text { Fluorescence Imaging }}^{a}$ & $<0.1 \mathrm{~mm}$ & $2 \mathrm{D}$ & $\sim 1000$ fps $^{a}$ \\
\hline Ultrasound Imaging $^{b}$ & $\mathrm{~cm}$ & $2 \mathrm{D} / 3 \mathrm{D}$ & $\sim 500 \mathrm{fps}^{b} / \sim 10 \mathrm{vps}$ \\
\hline Magnetic Resonance Imaging & $\mathrm{cm}$ & $2 \mathrm{D} / 3 \mathrm{D}$ & $\sim 50 \mathrm{fps}$ \\
\hline Optical Coherence Tomography & $<2 m m$ & $2 \mathrm{D} / 3 \mathrm{D}$ & $\sim 500 \mathrm{fps}^{c} / \sim 10 \mathrm{vps}$ \\
\hline Transillumination $^{a}$ & $<1 m m$ & $2 \mathrm{D}$ & \\
\hline
\end{tabular}

${ }^{a}$ mapping of surface at frame rates of up to $<10 \mathrm{kHz}$ with spatial resolutions of $100 \times 100$ pixels, ${ }^{b} \mathrm{~B}$-mode ultrasound imaging with A-scan rates of about $f_{A}=50 \mathrm{kHz}$ producing images of size $200 \times 200$ pixel, ${ }^{c}$ at A-scan rates of $f_{A}=$ $300 \mathrm{kHz}$

and visualization techniques. This requires imaging technology that is able to capture the volumetric tissue structure in a tomographic imaging mode. In conclusion, to image both function and structure of the heart, as well as different functional parameters simultaneously, multiple imaging modalities are required to be combined.

The rapidly evolving electrical wave pattern is typically recorded using imaging technology that produces two-dimensional movie data of the activity at appropriate acquisition speeds. Ideally, one would be able to capture the time-varying three-dimensional activity of the entire muscle at high speeds. However, such technology does not exist and it would be difficult to process the vast resultant information rates. In summary, nowadays fluorescence and ultrasound imaging are the goldstandard techniques for imaging of cardiac activity and are used very frequently in cardiac research. Both imaging modalities offer the possibility to be used as high-speed $(\sim 500 \mathrm{fps})$, high-resolution $(200 \times 200$ pixels, appropriate spatial resolution) imaging modalities and can together be used to measure electrophysiological and mechanical activity. Both technologies were accessible for this work and were the favored imaging modalities for the aims of this work, see following sections. Table 3.1 presents a list of imaging technology together with ist critical parameters regarding the acquisition speeds and performance.

\subsection{Optical Fluorescence Imaging}

Fluorescence imaging is widely used in cardiac research ${ }^{74,98,99}$ and was also extensively used in this thesis, see chapter 6 . Fluorescence imaging allows the visualization of various physiological processes in biological cells and tissues and is very frequently applied to image neuronal and cardiac cells. In cardiac research, fluorescence imaging is commonly referred to as optical mapping, eluding to its capability of mapping physiological activity also over larger surfaces of tissues at high spatial and temporal resolutions, enabling scientists to visualize, for instance, action potential wave patterns propagating over the epicardial surface of the ventricles. A review of the state-of-the-art of optical mapping techniques is given by Herron et al. ${ }^{213}$

Fluorescence imaging employs wavelength-dependent light-tissue interactions. Visualization of intramural activity is therefore severely limited by the penetration depth of the light through tissue. As penetration depths of light in biological tissues typically range below $<1 \mathrm{~mm}$, fluorescence imaging can not simply be performed transmurally ${ }^{47}$ through entire organs such as the heart muscle or in the presence of opaque materials like blood. The non-contact optical imaging modality offers numer- 
Chapter 3. Imaging of Electromechanical Wave Activity in the Heart

\begin{tabular}{lccc}
\hline dye & excitation peak & emission peak & parameter sensitivity \\
\hline Di-4-ANEPPS & $488 \mathrm{~nm}$ & $605 \mathrm{~nm}$ & $V_{m}$ \\
Di-8-ANEPPS & $468 \mathrm{~nm}$ & $635 \mathrm{~nm}$ & $V_{m}$ \\
Annine 6 & $450 \mathrm{~nm}$ & $515 \mathrm{~nm}$ & $V_{m}$ \\
Di-4-ANBDQPQ & $640 \mathrm{~nm}$ & $750 \mathrm{~nm}$ & $V_{m}$ \\
Rhod-2AM & $550 \mathrm{~nm}$ & $571 \mathrm{~nm}$ & {$\left[\mathrm{Ca}^{2+}\right]_{i}$} \\
Fluo-3 & $480 \mathrm{~nm}$ & $535 \mathrm{~nm}$ & {$\left[\mathrm{Ca}^{2+}\right]_{i}$} \\
Fluo-4 & $494 \mathrm{~nm}$ & $516 \mathrm{~nm}$ & {$\left[\mathrm{Ca}^{2+}\right]_{i}$} \\
\hline
\end{tabular}

Table 3.2: Fluorescent dyes commonly used in optical mapping experiments with cardiac tissue: voltage- $\left(V_{m}\right)$ and calcium-sensitive $\left(\left[\mathrm{Ca}^{2+}\right]_{i}\right)$ dyes

ous advantages over the use of ECG electrodes, among which is the high spatial resolution and the prevention of electrophysiological artifacts and mechanical distortions introduced by the electrodes being inserted into the tissue. Fluorescence imaging was used for the visualization of cardiac arrhytmias such as ventricular or atrial tachycardia or fibrillation in intact, isolated whole hearts ${ }^{44}$ as well as in monolayer cardiac cell culture preparations. ${ }^{115,136,146,154}$

\subsubsection{Fluorescent Probes}

Fluorescence imaging employs light to both illuminate and excite fluorescent probes introduced to the tissue. The probes can either be introduced to the intracellular space or may attach to the cell membrane and - once excited with light at the right wavelength - exhibit fluorescence ${ }^{18,20}$. Depending on physiological changes of the cell the fluorescence undergoes variations in its intensity or spectrum and these changes can be captured using optical filtering techniques and cameras. Currently, fluorescent probes are most commonly used to report transmembrane potential $V_{m}$ and intracellular Calcium concentration $\left[\mathrm{Ca}^{2+}\right]_{i}$, however, also magnesium, sodium, potassium, $\mathrm{pH}$, nitric oxide, redox state and oxygen content can be reported. ${ }^{213}$ The fluorescent probes can either be introduced to the tissue shortly before the measurement by staining the tissue preparation with dyes appropriately or can already be present inside the tissue at the time of measurement in genetically modified tissues which express auto-fluorescence. Auto-fluorescence occurs for instance in transgenic cardiac tissue containing green fluorescent proteins (GFP) or cyan fluorescent proteins (CFP). Fluorescent dyes can be characterized by their parameter sensitivity, their excitation and emission bandwidths, their response times and fractional fluorescence intensity changes with respect to electrophysiological changes. The most commonly used dyes are styryl dyes, of which aminonaphthylethenylpyridnium (ANEP) dyes are the most prominent ones. Throughout this work, the dye di4-ANEPPS was used. A list of fluorescent dyes together with their excitation- and absorption- and emission- peak wavelengths and the parameter sensitivity, that is the sensitivity to the membrane potential or the intracellular calcium concentration, is given in table 3.2. Each dye is commonly used in optical mapping experiments with cardiac tissue. In whole-heart optical mapping experiments, the tissue is stained by injecting fluorescent dye into the aorta from which it is distributed to the tissue via the vascular system, see chapter 6 .

\subsubsection{Imaging Hardware}

The hardware used for fluorescence imaging consists of illumination, optical filtering equipment, lenses and typically high-speed cameras and acquisition hard- and software. High-speed cameras are 
used to detect the fluorescence light. Most commonly used detectors are photodiode arrays (PDA), charged-couple device (CCD) and complementary metal oxide semiconductor (CMOS) cameras, each of which possess their own advantages and disadvantages. With the detector arrays it is possible to acquire simultaneously temporal electrophysiological signals from $\sim 10.000$ sites with temporal resolutions of $<1 \mathrm{~ms}$. For instance, the SciMedia MiCAM ULTIMA (Brainvision, Japan) CMOS camera operates with a spatial resolution of $100 \times 100$ pixels at a temporal resolution of $0.1-10 \mathrm{kHz}$. The Photometrics Cascade CCD camera operates with a spatial resolution of $128 \times 128$ pixels at a temporal resolution of $0.1-0.5 \mathrm{kHz}$. The optical setup of an optical mapping system consists of objectives with low numerical aperture to direct the excitation light onto and collect in the same time emitted fluorescence light from the tissue. Furthermore, a set of bandwidth barrier filters and dichroic filters is used to separate excitation light from emission light in the optical path of the optical mapping system. Therefore, it is guaranteed that only fluorescence reaches the detector. Fluorescence imaging can be applied to single cells and groups of cells at cellular and subcellular levels as well as to whole populations of cells at the whole heart organ level. Here, the focus is set on the macroscopic approach with large fields of view ranging from $1 \times 1 \mathrm{~mm}^{2}$ to $3 \times 3 \mathrm{~cm}^{2}$. Fluorescence imaging can be used in mono-parametric as well as in multi-parametric imaging mode, capturing multiple physiological parameters, such as transmembrane potential and intracellular calcium transients, simultaneously. Fluorescence imaging can also be used in a panoramic fashion, imaging the cardiac cell culture preparations with multiple cameras from multiple directions.

\subsubsection{Post-Procesing}

Fluorescence imaging captures small fractional changes in fluorescence intensity that are immediately caused by physiological activity of the tissue. Common techniques to visualize the small fractional changes in the fluorescence intensity $I_{f}(t)$ are to normalize the temporal signal in each pixel over time or to subtract its mean over time from the signal itself, thus amplifying the intensity variations. This can be done under the assumption that each pixel $\vec{p}(x, t)$ continuously shows the same site of the tissue. Spatial gaussian, box car or cone filtering may be applied to improve signal-to-noise ratio. In case the tissue exhibits motion and the recorded frames show different tissue configurations $\chi_{t}$ the fluorescence variations of each site can not be associated with each pixel any longer, see section 6.4. The image contains motion artifacts. Then, the motion needs to be registered postacquisition and each site exhibiting fluorescence needs to be tracked over space and time before the aforementioned analysis can be applied.

\subsubsection{Clinical Applications}

There are no clinical applications using conventional fluorescence imaging as the involved chemical compounds in fluorescent dyes are toxic. However, the field of optogenetics explores the possibility of creating transgenic tissue, with which, in principle, non-invasive fluorescence imaging should be possible. 


\subsection{Ultrasound: Echocardiography}

Ultrasound imaging is widely used in cardiac research and was also extensively used in this thesis, see chapter 6. Ultrasound is a tomographic imaging technique typically used to penetrate biological tissues. It is referred to as sonography when applied in the medical field or echocardiography when imaging the heart.

\subsubsection{Fundamentals}

Equal to acoustic sound, ultrasound is a longitudinal cyclic pressure wave. The frequency range corresponding to ultrasound is $20 \mathrm{kHz}-200 \mathrm{M} \mathrm{Hz}$. Ultrasound imaging employs the speed of sound to measure distances. Therefore, the echo delay time $\tau$, obtained from a sound pulse traveling through tissue and being reflected at different depths back to its origin, together with its propagation speed, that is the speed of sound in biological tissue, determines the distances of sites to the origin from where the sound wave was emitted. The sound wave is emitted and detected by a piezoelectric transducer, which is a crystalline or ceramic disk that vibrates if an alternating voltage is placed across it. In turn, mechanical vibrations experienced by the transducer are converted into a voltage, enabling the detection of backreflected acoustical waves. As a result, we obtain distances of sites in the form of an intensity profile of echoes along the one-dimensional linear propagation direction of the sound wave. Such a linear scanning line is referred to as an A-scan.

Sound waves have many similar physical properties to light, including diffraction, scattering and interference. Diffraction and scattering occur due to acoustic impedance, an effect correlated to a variation of the ratios of the density of the medium and the velocity of sound inside the medium, analogeous to the refractive index $n$ in the interaction of light with materials. Sound waves can be described with methods from linear acoustics. Pressure wave fields are assumed to represent solutions of the one-dimensional linear wave equation in time $t$ and position $x$ :

$$
\frac{\partial^{2} p}{\partial x^{2}}=\rho_{0} \kappa \frac{\partial^{2} p}{\partial t^{2}}
$$

where $p$ is the pressure, $\rho_{0}$ is the medium density at equilibrium, and $\kappa$ is the compressibility. Ultrasound imaging requires a transducing medium with a high enough density to support the propagation of sound waves. Ultrasound imaging can not be performed in air. The velocity of sound in biological tissue is $1540 \frac{\mathrm{m}}{\mathrm{s}}$. In ultrasound imaging, the sound wave travels into the tissue and comes into focus at a desired depth. The tighter the focused beam's waist, the greater the transversal or lateral resolution. The shorter the pulse duration or the higher the ultrasound frequency, the greater the axial resolution. The axial direction indicates the predominant direction of sound propagation, extending from the transducer into the tissue. The attenuation of the sound wave is increased at higher frequencies. Consequently, better penetration into the tissue comes with coarser spatial resolution. High frequency ultrasound imaging systems operating with spatial resolutions of $\sim 20 \mu \mathrm{m}$ and a frequency of $40 \mathrm{M} \mathrm{Hz}$ offer penetration depths of a few $\mathrm{mm}$ into tissue.

\subsubsection{Imaging Modes: Two- vs. Three-Dimensional Imaging}

Typically, echocardiographic imaging is associated with two-dimensional, cross-sectional ultrasound imaging where the two-dimensional ultrasound images are created by the repeated, subsequent acquisition using so-called $A$-scan beams. Scanning is achieved using motors and rotational scanning with 


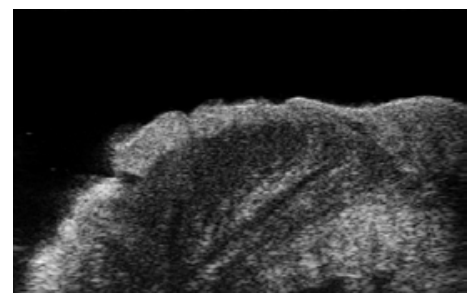

(a)

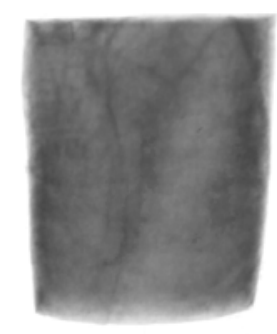

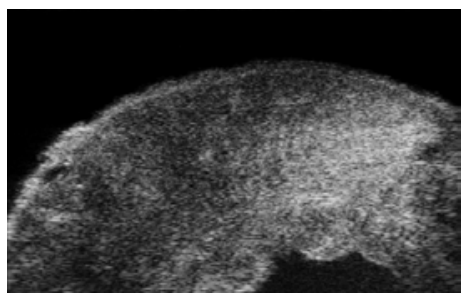

(b)

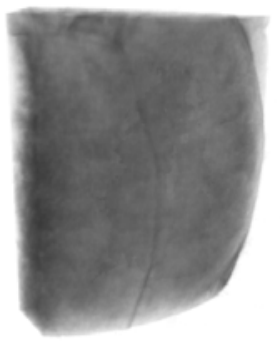

(d)

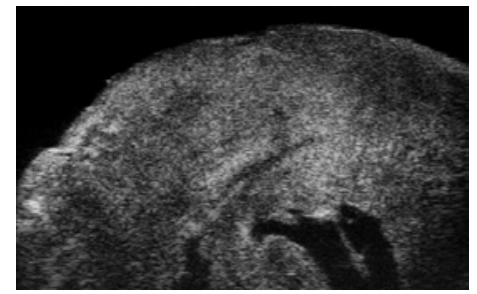

(c)

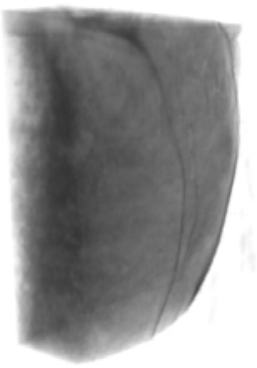

Figure 3.1: Ultrasound scan of left ventricular wall of formalin-fixed rabbit heart: volumetric scan obtained in pseudo-three-dimensional imaging or compounding mode with multiple repeated B-mode scans (500 images) used to reconstruct the volume. Sequence of B-mode images was acquired using a motorized translation stage. Panels (a-c) show raw ultrasound speckle image frames 0,80 and 288 from within the acquired stack. Panel (d) shows volumetric rendering of the entire stack of images with slightly transparent opacity transfer function. Speckle image width: $14.08 \mathrm{~mm}$

one transducer crystal or phased-array technology with many ultrasound transducer crystals, beam forming, focusing and sweeping. Figure 3.1 depicts two of the different imaging modes that are typically provided by ultrasound imaging. The upper panel shows typical two-dimensional speckle images or B-mode scans. Several repeated, subsequent A-scan lines placed with lateral shift in one line next to another can be used to create two-dimensional, cross-sectional, tomographic scans of tissue, referred to as $B$-scans. Hence, tissue motion can be imaged with ultrasound when repeated $\mathrm{B}$-scans are acquired from the same cross-section of the tissue. Ultrasound imaging allows tissue motion to be imaged as a sequence of two-dimensional B-scans at high-speeds with temporal resolutions of $<10 \mathrm{~ms}$, see chapter 6 . Conventional cross-sectional, two-dimensional ultrasound imaging is extended to ultrasound compounding or 3-dimensional ultrasound mosaicing, a technique in which adjacent, two-dimensional, cross-sectional B-scans are used to reconstruct volumetric tomography data. ${ }^{54,155}$ The imaging plane is then swept mechanically in transversal direction or the B-scans are collected during freehand ultrasound imaging. However, ultrasound compounding techniques are limited to volumetric acquisitions of static tissue structures. Intravascular echocardiography utilizes specialized catheters to image artery walls from within the vascular system with the aim to visualize atheromatous plaque in the coronary arteries. ${ }^{29}$ Here, the term pseudo-three-dimensional imaging is used to distinguish between volumetric imaging which is achieved via repeated and compounding techniques and true three-dimensional, volumetric ultrasound imaging. Three-dimensional ultrasound imaging produces volumetric three-dimensional tomographic data. Three-dimensional ultrasound imaging was first developed in the 1960s, and applied to the heart in the 1970s. ${ }^{143}$ The three-dimensional visualization of dynamic structures in real-time was demonstrated by Ramm et $a l .{ }^{33}$ Real-time three-dimensional transesophageal echocardiography, which permits the acquisition 
and visualization of the beating heart in thre spatial dimensions is described by Baltzer et al. ${ }^{160}$ The described ultrasound imaging system (Philips IE 33/Sonos 7500, X7-2t, Philips Healthcare, Andover, Massachusetts) operates with a phased matrix-array transducer with 2500 active elements which offers the acquisition of a pyramidal volume with an acquisition speed of $20-30 \mathrm{~Hz}$, which corresponds to a temporal resolution of $33-50 \mathrm{~ms}$. Spatial resolution of the system is worse in comparison to conventional two-dimensional ultrasound imaging systems.

\subsubsection{Clinical Applications}

Clinical applications of echocardiography are diverse. Conventional ultrasound imaging of the heart includes transthoracic echocardiography in which imaging is performed through the chest wall as well as transesophageal echocardiography in which a specialized probe containing an ultrasound transducer at its tip is passed into the patient's esophagus, allowing imaging in close proximity to the heart, see Pua et al. ${ }^{108}$ Quantitative measurement of ventricular volume, mass and ejectionfraction are important measures in clinical cardiac assessment and are typically also measured using ultrasound. ${ }^{169}$ Ejection-fraction is considered to be an almost universal measure for the contractile performance of the ventricular muscle ${ }^{169}$ and likewise a measure for the physiological or pathophysiological condition of the heart. Ultrasound can not only be used as a diagnostic medical tool but can also be used as an interventional therapeutical tool. High-intensity focused ultrasound ablation rises locally the temperature of a target tissue and therefore destroys it.

\subsection{Other Imaging Techniques}

\subsubsection{Magnetic Resonance Imaging}

Magnetic resonance imaging is a non-invasive high-resolution imaging technique. Magnetic resonance imaging is of interest in the context of this thesis as the developed techniques are in principle applicable to this imaging technique as well. Magnetic resonance imaging was recently used as a dynamic imaging modality to acquire sequences of two-dimensional cross-sectional scans of a beating heart at a $20 \mathrm{~ms}$ temporal resolution. ${ }^{195,196}$ The spatial resolution was $1 \mathrm{~mm} \times 1 \mathrm{~mm}$ with a field of view of $25 \mathrm{~cm} \times 25 \mathrm{~cm}$. Despite recent advances, dynamic imaging of three-dimensional volumes using magnetic resonance imaging at real-time speeds remains challenging. ${ }^{95,131,169}$ The penetration depth of magnetic resonance imaging is large, its spatial resolution about $\sim 1 \mathrm{~mm}^{3}$.

\subsubsection{Computerized Tomography}

Computerized tomography uses x-ray radiation with wavelengths in the order of $10^{-7} \mathrm{~m}$ to $10^{-9} \mathrm{~m}$ to penetrate biological tissue and produce tomographic scans. Computerized tomography is used for static, structural imaging of prepared hearts with very high spatial resolutions, large fields of view and penetration depths of many centimeters. ${ }^{16}$ Computerized tomography with micron resolution capabilities was used in this work to obtain three-dimensional structural scans of excised rabbit hearts, see figure 3.2 and section 4.4 in chapter 4 . Because the technique employs ionizing electromagnetic radiation, it is a less attractive candidate for the use for electromchanical wave imaging as ultrasound or magnetic resonance imaging. 


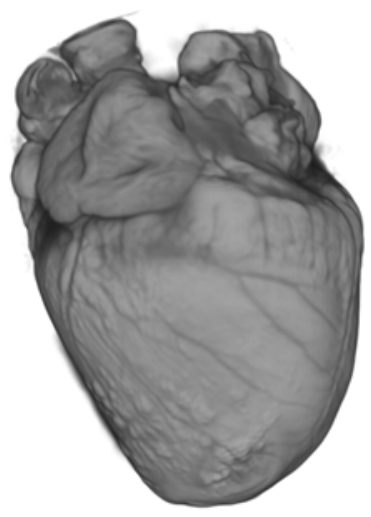

(a)

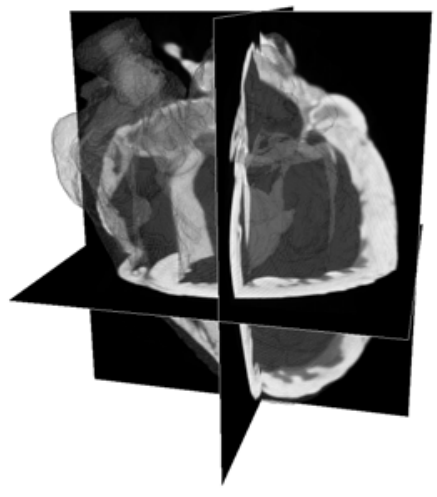

(b)

Figure 3.2: Computerized tomography scan of formalin-fixed rabbit heart with $\mu m$-resolution: (a) volume rendering with steep opacity transfer function showing outer surface of heart, (b) combination of orthoslices showing ventricular walls and chambers and translucent grey surface of tissue extracted from image segmentation

\subsubsection{Transillumination and Optical Tomography}

Transillumination and so-called diffusive optical tomography, multiplicative optical tomography ${ }^{138}$ and laminar optical tomography ${ }^{142}$ was introduced by Pertsov and co-workers. ${ }^{47,75,97,138,142,204}$ It refers to a series of experimental imaging techniques that were developed with the intend to visualize intra- or transmural electrical activity in the heart employing optical techniques. The techniques relate to optical tomography, and employ similarly as fluorescence imaging light-tissue interactions and fluorophores. Due to the low penetration depths of light, however, these techniques were not successful in providing in-depth visualizations of transient wave phenomena inside and throughout the heart walls. To achieve higher penetration depths, high-intensity focused light was used and directed together with scanning schemes into the tissue. Electrical activity was reported to have been imaged inside the ventricular wall of a mouse heart. ${ }^{142}$ However, the single light point scanning scheme lead also to slow acquisition speeds, making it impossible to capture non-repetitive transient wave phenomena. In the context of imaging cardiac activity, transillumination and optical tomography techniques are purely scientific . No clinical applications were reported.

\subsubsection{Electrode Recording}

Electrode recordings are a standard tool to access the electrophysiological activity of cardiac tissue. Spatio-temporal patterns of electrical activity can be recorded with hundreds of electrodes situated on the heart surface at high speeds with temporal resolutions $<1 \mathrm{~ms}$. Common implementations are multi-electrode arrays ${ }^{176}$ or ECGi. ${ }^{109,137}$ However, while the spatial resolution of these methods is low and no transmural information is provided, it is likely that the presence of the electrodes modifies the patterns of electrical activity. In many cases, optical mapping techniques are favored.

\subsubsection{Optical Coherence Tomography}

Optical coherence tomography (OCT) is a high-speed tomographic imaging modality employing light and interferometry techniques to obtain structural information of optically penetrable tissue 
volumes. OCT is typically used to obtain two-dimensional, cross-sectional or three-dimensional, volumetric data of biological tissue. OCT is especially suited for morphological tissue imaging due to its fine spatial resolution $(<10 \mu \mathrm{m})$ and its reasonable penetration depth of several $\mathrm{mm}$. The spatial resolution of OCT scans can be of comparable quality to histological cuts with sub micrometer resolutions. OCT is notably applied in ophthalmology where it is used to obtain detailed two- and three-dimensional structural scans of the eye, including cornea and retina, see Gora et al. ${ }^{179}$ OCT was also shown to provide sufficient acquisition speeds to acquire cardiac motion appropriately and be able to penetrate the ventricular wall of a mouse heart. ${ }^{199}$

\section{Fundamentals}

Analogous to ultrasound OCT uses the delay time $\tau$ in which a light wave propagates through the tissue and is backscattered by sites in the tissue to determine the distances of those sites. However, due to the speed of light, the delay time $\tau$ cannot be determined electronically, but is determined by interferometric techniques. OCT images are then generated based on the variation of optical tissue properties from different structures. An introduction to optical coherence tomography is given by Drexler et al. ${ }^{162}$ and others. ${ }^{66,82,124}$

The time delay $\tau$ is determined from the auto-correlation function $\Gamma(\tau)$ in the interference process of an electromagnetic light wave $E$ with itself. The basic setup of an OCT imaging system is a Michelson interferometer. A coherent, broad-bandwidth laser beam is split into a reference and a probe beam by a beamsplitter. The probe beam $E_{1}$ is directed into a tissue sample from where it is reflected back and reintroduced into the optical path and recombined with the reference beam. The reference beam $E_{2}$ stays in the Michelson interferometer. Both electromagnetic wave fields $E_{1}$ and $E_{2}$ interfere and we obtain a superposition of the two electromagnetic waves $E_{1}$ and $E_{2}$. The intensity $I_{D} \sim E^{2}$ on a detector is given by:

$$
\begin{aligned}
I_{D} & =<E_{1}{ }^{*} E_{2}> \\
& =I_{1}+I_{2}+\operatorname{Re}\left\{<E_{1}^{*} E_{2}>\right\} \\
& =I_{1}+I_{2}+\operatorname{Re}\{\Gamma(\tau)\}
\end{aligned}
$$

The result is an interference pattern which depends on the complex auto-correlation function $\Gamma(\tau)$. The electromagnetic light wave $E_{1}$ having traveled into the tissue and back experienced a delay of $\tau=\frac{2 d}{c}$ compared to the reference light wave $E_{2}$ that stayed in the interferometer. Actually, both electromagnetic waves are the same electromagnetic wave field as they were delivered by the same light source and we set $E_{1}=E_{2}=E$ :

$$
\begin{aligned}
I_{D} & =<E^{*} E> \\
& =2 I+\operatorname{Re}\{\Gamma(\tau)\}
\end{aligned}
$$

That is, a part of the electromagnetic light wave $E$ experiences a temporal shift $\tau$ and interferes with itself. The intensity on a detector depends on the time delay $\tau$. The complex auto-correlation 
function, also referred to as complex self-coherence function, ${ }^{92}$ is given by:

$$
\Gamma(\tau)=\lim _{T \rightarrow \infty} \frac{1}{T} \int_{-\frac{T}{2}}^{\frac{T}{2}} E^{*}(t) E(t+\tau) d t
$$

For a harmonic, monochromatic, linear wave $E(t)=E_{0} e^{-i \omega t}$ the complex self-coherence function $\Gamma(\tau)=\left|E_{0}\right|^{2} e^{-i \omega t}$ depends harmonically on the time delay $\tau$. Furthermore, the intensity on the detector $I_{D}(\tau)=2 I(1+\cos (\omega \tau))$ depends harmonically on the time delay $\tau$ which depends in turn on the distance $d$ that the probe beam needed to travel through the tissue. When translating the end mirror of the reference arm of the Michelson interferometer and subsequently shifting the reference light wave against the probe light wave we would repeatedly obtain destructive and constructive interference alternating periodically with the wavelength of the light. This means, that with completely coherent light one would not be able to obtain depth resolved tissue information by scanning through the tissue. OCT generally employs broad bandwidth light with a short coherence length $l_{c}$. This results in an interference signal on the detector with gaussian input:

$$
\begin{aligned}
I_{D} & \sim \operatorname{Re}\{\Gamma(\tau)\} \\
& =\operatorname{Re}\left\{e^{-i \omega_{0} \Delta \tau} e^{-\frac{\sigma_{\omega}^{2} \Delta \tau^{2}}{2}}\right\}
\end{aligned}
$$

The auto-correlation function of a gaussian function is again a (modulated) gaussian function. Consequently, when employing light with a short coherence length $l_{c}$ we obtain constructive interference only when the length of the reference arm matches the distance $d$ that the light travelled in the probe arm. Consequently, when scanning the reference arm we can determine the distance of the site in the tissue that reflected the probe beam back into the Michelson interferometer. The distance is indicated by the one distance at which constructive interference occurs.

\section{Technological Implementations}

OCT imaging systems are presently realized in a variety of technological implementations. The majority of OCT imaging systems employ single scanning point or confocal scanning schemes. They use a single focused laser beam to construct a cross-sectional B-scan or a volumetric scan from subsequent, adjacent A-scans. The beam is directed into the tissue via galvanometer scanners which can be programmed to scan arbitrary scanning patterns. A time-domain based OCT system employs a mechanically translated end mirror in the reference arm to temporally shift the light against itself and to scan the different delay times $\tau$ and distances $d$ in axial direction. A-scan rates of $f_{A} \approx 0.1 \mathrm{kHz}-10 \mathrm{kHz}$ are typically achieved with time-domain based OCT and acquisition speeds are limited by the mechanical scanning technique.

Parallel or full-field OCT employs a different scanning scheme together with time-domain based OCT. Instead of scanning a single laser beam through the tissue the sample is full-field illuminated and en face imaged with a 2-dimensional CCD detector, hence acquiring many neighboring sites in parallel during one mechanical axial scan. Newer technological implementations of OCT imaging systems employ different, faster axial data acquisition techniques. In particular, the mechanical axial scanning technique is avoided. 


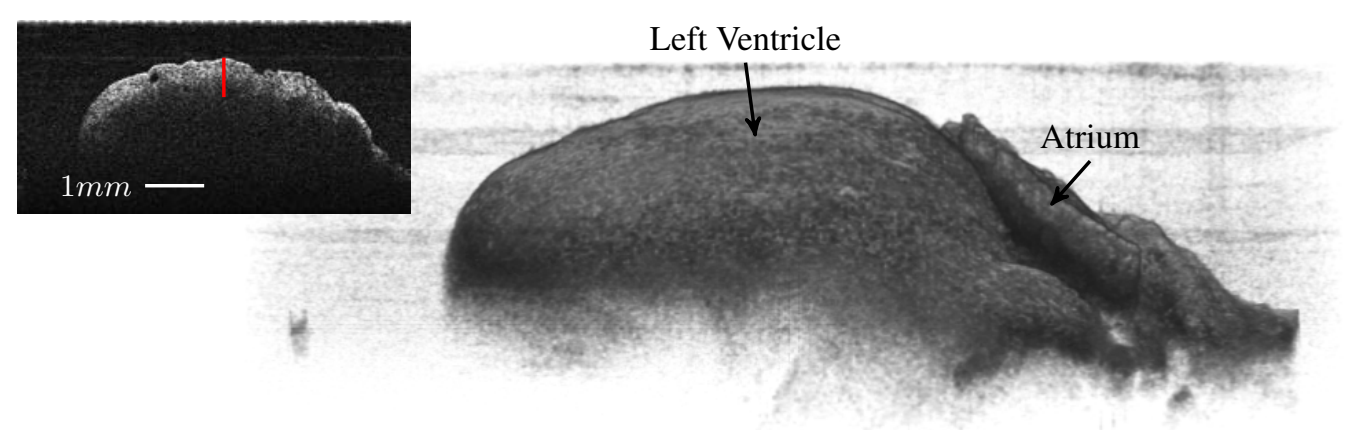

Figure 3.3: Mouse heart volumetric scan acquired with optical coherence tomography: high-speed FDMLOCT system operating in the near-infrared at wavelengths of $\lambda=1300 \mathrm{~nm}$. Due to the long wavelength the beam was found to be able to penetrate almost the entire thickness of the ventricular wall (indicated by red line) of the mouse heart.

In Fourier-domain based OCT systems the axial depth information is encoded in frequency space rather than in time. The axial depth information can be calculated via the Wiener-Khintchine theorem between the auto-correlation function and the spectral power density by a Fourier-transform of spectra acquired when light with different wavelengths is backscattered from the tissue. One implementation of a Fourier-domain OCT imaging system is Swept-Source (SS) OCT. In SS-OCT the frequency is encoded in time as a scanning light source repeatedly sweeps over a broad bandwidth spectrum and the backscattered light is recorded sequentially by a photodiode. Instead of mechanically scanning with the reference arm through the tissue depth in axial direction, a frequency bandwidth is scanned through and its single frequencies come to a constructive interference at different tissue depths. The wavelength having caused the constructive interference can be correlated with the spatial distance $d$ of the site having backscattered the light. One sweep of the light source corresponds to one A-scan. A-scan acquisition speeds can be increased dramatically as the sweeping speed of the light source is faster than the mechanical translation of the reference arm. Another implementation of a Fourier-domain OCT imaging system is Spectral-domain (SD) OCT. In SD-OCT the full bandwidth of the broad bandwidth light source is sent into the probe arm at once and the single frequencies are spatially separated by a dispersive element before they are captured by a linear detector (CCD/CMOS) array. Broadband interference is acquired with spectrally separated detectors from which each detector captures a frequency range corresponding to a spatial depth range in axial direction. Accordingly, the number of used elements of the detector determines the axial spatial resolution. Fourier-domain OCT imaging systems operate with a single scanning point scanning scheme.

\section{Performance: Speed and Penetration Depth}

A-scan acquisition speeds can be increased dramatically using Fourier-domain OCT compared to time-domain based OCT as the read-out rates of the line scan camera detectors determine the A-scan rates of SD-OCT imaging systems. SD-OCT imaging systems typically operate with A-scan rates of $f_{A} \approx 10 \mathrm{kHz}-100 \mathrm{kHz}$. The latest development in Fourier-domain OCT imaging is the use of FDML (Fourier Domain Mode Locking) lasers as light sources. ${ }^{126}$ FDML lasers are ideal rapidly 
sweeping light sources for OCT applications. They combine high repetition rates of conventional mode-locking lasers at a constant output power with a spectral chirp, and allow higher sweeping rates than conventional swept-sources. Consequently, FDML SS-OCT imaging systems are very promising candidates for high-speed applications of OCT imaging. Single scanning point high-speed OCT systems based on FDML technology can achieve $A$-scan repetition rates of $f_{A}=300 \mathrm{kHz}$. This translates to frame rates of about $f_{f p s}=100-1000 \mathrm{~Hz}$ when scanning in two-dimensional imaging mode. The penetration depth of OCT is limited to a few millimeters in optically dense tissue as cardiac muscle tissue.

\section{Clinical Applications}

Clinical applications of OCT are limited to cardiovascular tomography. Intravascular OCT is a cost effective and more practical alternative to intravascular echocardiography. ${ }^{152}$ Transmural imaging of the heart is limited to single applications in cardiac research, see. ${ }^{99}$ Elastographic imaging was demonstrated using OCT together with speckle-tracking techniques. ${ }^{63}$ However, elastographic imaging was not applied to cardiac tissue. The functional use of OCT in neuronal tissue was demonstrated by ${ }^{94}$ It was observed that optical scattering increases in electrically stimulated axons. Consequently, OCT is able to detect optical changes in cells and tissue during physiological processes. OCT typically employs near-infrared light as scattering effects are less with longer wavelengths and the achieved penetration depth into optically dense tissue is larger. The optimal wavelengths for applications in cardiology range from $800 \mathrm{~nm}-1500 \mathrm{~nm}$ and are referred to as the therapeutical window. ${ }^{152}$

\subsubsection{Other Imaging Techniques}

There is a bewildering landscape of imaging techniques and it is possible to identify many more techniques which have a certain relevance with respect to this work. Magnetic particle imaging uses the distribution of superparamagnetic iron oxide particles to acquire dynamic three-dimensional tomographic data of cardiac tissue. ${ }^{17,187}$ Temporal resolutions of $\sim 20 \mathrm{~ms}$ with three-dimensional fields of view of $20 \mathrm{~mm} \times 12 \mathrm{~mm} \times 17 \mathrm{~mm}$ with a spatial resolution sufficient to resolve all heart chambers have been reported. Spatial resolutions are in the order of $\sim 1 \mathrm{~mm}^{3}$. Photoacoustic imaging is a hybrid imaging modality consisting of a combination of ultrasound and optical imaging. It employs the photoacoustic effect in which non-ionizing laser pulses are used to deposit heat energy in tissue and to stimulate a transient thermoelastic expansion with a consequential wideband ultrasonic emission. Acquisiton speeds are therefore mostly bound to the acquisition speeds of the ultrasound detection system. B-scan acquisition speeds of $f_{B}=166 \mathrm{~Hz}$ with axial and lateral resolutions of $25 \mu \mathrm{m}$ and $70 \mu m$ respectively and penetration depths as deep as several $\mathrm{mm}$ were reported. ${ }^{185}$ Terahertz imaging employs electromagnetic light to penetrate matter. Terahertz imaging is much less affected by scattering due to its long wavelengths and would likely penetrate much further than infrared light into tissue. However, the high absorption of terahertz radiation by water limits the penetration depth to $<1.5 \mathrm{~mm}$ in biological tissues with high water contents. As of today, terahertz imaging is limited to applications in dermatology and dentistry. ${ }^{101}$ A variety of optical tomography imaging modalities have been developed, some of them with the particular aim to visualize the electrophysiology within the 3-dimensional cardiac muscle. Confocal microscopy is a typical example of a widely used optical tomography imaging modality, which provides high spatial resolutions of just a few micrometers. As this technique only illuminates one point in the tissue at a time, 2-dimensional or 3-dimensional imaging requires scanning over a regular pattern. This is a typical feature of most optical tomography 
imaging modalities. However, confocal microscopy typically penetrates much less deeply, that is in the order of $<100 \mu \mathrm{m}$, into tissue than the desired penetration depths of several $\mathrm{mm}$. This limits its relevance to applications in transmural imaging of the heart. Macroconfocal imaging uses a combination of confocal microscopy and laser scanning techniques to assemble raster images of tissue volumes. Acquisition times for acquiring the scans is in the order of minutes.

\subsection{Computational Techniques: Image Registration}

Image registration is a computational technique from the field of computer vision used to automatically interpret various types of image or video data. In the medical field, image registration is most commonly used to identify correlations, shapes or features inside image or video data with the aim to reconstruct motion or deformation or to match shapes or volumes from different data sets onto each other. Practical applications of image registration in the biomedical field are very diverse. Typically, it is a common task to match two data sets obtained from two different imaging modalities. This can also involve the matching of three-dimensional medical images. ${ }^{110}$ A review of image registration techniques is given by Maintz et al. ${ }^{61}$ In this thesis, image registration was used to identify and track motion inside movies showing contracting heart tissue. Generally, the registered data can be two- or three-dimensional as well as static or time-varying data. Here, all registration applications were limited to dynamic two-dimensional data, see chapter 6 . Image registration was used for two purposes: (1) to register motion inside video data and (2) to subsequently reconstruct deformations of the tissue configuration and to extract quantitative information about the deformation.

\subsubsection{Correlation-based Motion Tracking}

Correlation-based block-based motion tracking refers to computational image registration techniques, that directly measure the correlation or similarity between neighbouring image subregions of two images to compute the optical flow between two images. The techniques employ optical properties of the image data, assuming that motion is reflected by the motion of the grayvalue or intensity pattern within the image that i showing the motion. The optical flow consists of displacements that can be assigned to every pixel of the image, derived from the shifts that were detected in between two compared images. For instance, particle image velocimetry is a spatial correlation analysis technique used to register speckle motion in a sequence of images. ${ }^{151}$ The technique is usually applied in the field of fluid dynamics to detect flow inside liquids or gases. In conventional particle image velocimetry, tracer particles are introduced to a flow and a two-dimensional cross-section of the medium is illuminated with light. The flow of tracer particles within the light sheet is usually recorded with high-speed cameras and the sequence of images is then spatially correlated to each other under the assumption that tracer particles move coherently and uniformly in between two images and that their displacement is sufficiently small to be captured within subregions of the images. In the case of block-matching techniques, for evaluation of spatial correlation the images are divided into small subregions usually referred to as interrogation windows. For each interrogation window the local displacement $\vec{u}$ is determined by means of spatially correlating the image data of the site under consideration with the optical properties of its surrounding. The process of interrogation is repeated for all interrogation windows of the images, such that we obtain a displacement map for each image with several hundreds to thousands of instantaneous displacement vectors. The statistical methods employ cross-correlation or minimum quadratic difference algorithms. The displacement vector $\vec{u}$ then points into the direction of the minimum of the 2-dimensional correlation maps. Due to the 
planar illumination of the flow field only 2 in plane components of the displacement vector $\mathbf{u}$ can be determined. This is referred to as two-component particle image velocimetry. Spatial correlation techniques used in particle image velocimetry can be applied to many other forms of data and is not limited to particle images. In this study, it was applied to vidoe data as well as cross-sectional ultrasound B-scan speckle images showing contracting myocardial tissue. Gray value intensity distributions rather than particles were tracked. In chapter 6 , the local displacements $\vec{u}$ were calculated from interrogation windows in between 2 speckle images using the minimum quadratic differences method $^{53}(\mathrm{MQD})$. The average displacement $(\Delta x, \Delta y)^{t}$ of two interrogation windows $\mathcal{S}$ and $\mathcal{S}^{\prime}$ can be found by determining the minimum of the quadratic difference. The location of the minimum value in the two-dimensional correlation plane given in equation (5.5.2) is used as the displacement $\vec{u}$. The accuracy of cross-correlation and MQD algorithms are similar. MQD algorithms are in general slower than cross-correlation algorithms. However, MQD algorithms may be more robust for speckle images. Cross-correlation or mean-quadratic-difference methods inherently recover only linear displacements $\vec{u}$, that is the average linear shift of the intensity distributions within the interrogation window. Rotations or deformations can only be recovered by further processing of the displacement maps. The interrogation window size needs to be chosen sufficiently small such that second order motion effects can be neglected. The presented spatial correlation technique can track tissue motion in a sequence of speckle images, see chapter 6 .

A different spatial correlation technique, which can be used for image registration and motion registration of tissue was introduced by. ${ }^{21}$ The Lucas-Kanade method is a locally affine, globally smooth transformation based on optical flow estimation using spatial intensity gradients of images. Assuming a constant flow in a local neighborhood of a pixel, the method solves the basic optical flow equations for all the pixels in that neighbourhood by the least squares criterion. If $I_{0}(x, y)$ and $I_{t}(x, y)$ denote a reference and a deformed image, an affine mapping is given as follows: ${ }^{70}$

$$
I_{0}(x, y)=I_{t}\left(m_{1} x+m_{2} y+m_{5}, m_{3} x+m_{4} y+m_{6}\right)
$$

where $m_{i}$ with $i=1, \ldots, 6$ are parameters of the affine mapping. The following quadratic error function $E(\mathbf{m})$ then needs to be minimized in order to find the best match between reference and deformed image:

$$
E(\mathbf{m})=\sum_{x, y \in \Omega}\left(I_{0}(x, y)-I_{t}\left(m_{1} x+m_{2} y+m_{5}, m_{3} x+m_{4} y+m_{6}\right)\right)^{2}
$$

where $\Omega$ defines the spatial region of interest in the image. This generally nonlinear function is linearized using a first-order truncated Taylor series expansion. The numerical scheme developed by ${ }^{70}$ solves the approximate error function in Newton-Raphson iterative steps, where in each iterative step the affine parameters are estimated and the current image is repeatedly warped to the reference image. The Lucas-Kanade method assumes constant brightness of the images.

Determining dynamical elastic properties is generally referred to as elastography. Elastography experiments can generally be performed with optical imaging, ultrasound and optical coherence tomography. Ultrasound elastography was introduced by Ophir et al. ${ }^{37}$ Elastography typically refers to experimental techniques, however, they are often combined with computational techniques to capture elastic deformations. ${ }^{71,91}$ 
Chapter 3. Imaging of Electromechanical Wave Activity in the Heart

\subsection{Visualization}

Imaging of the heart also requires appropriate visualization of the data. The visualization procedure using computer graphics techniques can become a non-trivial and highly specialized task ${ }^{149}$ because of the motion and deformations of the to be visualized physical activity. Visualizing threedimensional time-varying simulation output data can be achieved using various readily available software solutions. Typically, volume data as output of numerical simulations or experimental measuring techniques such as computerized tomography or macroconfocal laser scanning techniques is organized in a voxel-structure on a regular rectilinear grid. The visualization of time-varying three-dimensional dynamics is then the subsequent visualization of three-dimensional voxel data or matrices for each time step, using ray-tracing or other volume rendering or visualization techniques, with the overall data corresponding to four-dimensional image data. However, the output of moving and deforming media does not necessarily provide a regular grid or voxel structure. In this thesis, a custom visualization technique had to be implemented because readily available software for the visualization of the data was not available. The procedure involved also re-rasterization and voxelization of the simulation grid structure into regular three-dimensional image data. For this task The Visualization Toolkit (VTK) was used. 
Chapter 3. Imaging of Electromechanical Wave Activity in the Heart 


\section{Chapter 4}

\section{Computational Model of Heart Tissue with Mechano-Electric Coupling}

To study coupled electromechanical wave activity in cardiac muscle tissue, see chapter 5, a computational model allowing to simulate the multi-physical coupled electrical and mechanical behavior of cardiac tissue was developed. Cardiac tissue undergoes large deformations due to the contractions of cardiac muscle cells, cardiomoycytes, which contract upon electrical excitation, see chapter 1 and 2. The electrical excitation that spreads inside the multi-cellular cardiac substrate and elicits the contractions of cardiac cells was aimed to be depicted on the macroscopic, whole-organ level by the diffusive spread and propagation behavior of nonlinear waves of excitation. These waves exhibit reentry and a scroll wave-like organization during chaotic, fibrillatory cardiac activity, see also section 1.3.2 in chapter 1 and section 2.1.5 in chapter 2.

One of the goals of this thesis was to study elasto-mechanical deformation patterns and contractile motion of myocardial muscle tissue during chaotic electrical scroll wave activity in-silico, see chapter 5. Results were supposed to guide the planning of experiments with real cardiac tissue preparations as well as enable interpretation of the measurements. The computational model was required to be able to simulate coupled electromechanical scroll wave activity inside large actively contracting and deforming tissue volumes at acceptable computational speeds, taking into account the highly anisotropic properties of myocardial muscle tissue. This chapter describes the computational methods developed for these purposes. The computational model and numerical implementation was developed by adapting multiple different, readily published concepts for the different parts of the multi-physical problem and combining them to be used in one computational framework for the modeling of the multi-physical processes. The desired efficiency guided the choice of computational techniques. For instance, the finite-element method, which is widely used for soft-tissue modeling, was avoided due to its heavy computational demands and high technical requirements. Alternatively, finite differences and particle systems approaches were found to provide efficient numerical methods, which were yet accurate and performant enough to simulate many phenomena of cardiac tissue qualitatively. The computational model developed in this work provided the proto-type that was used at later stages of this thesis as the basis for further developments leading to a computational framework allowing to simulate coupled electromechanical wave activity inside realistically heart-shaped cardiac tissues within the scope of a Master's thesis project, see sections 4.1.1, 4.3 and 4.4. 


\subsection{Modeling of Electrophysiological Excitation Patterns}

To simulate waves of electrical excitation, both the phenomenological three-variable Fenton-Karma ${ }^{58}$ model as well as the phenomenological two-variable Aliev-Panfilov ${ }^{51}$ model were used, see section 2.1.5 in chapter 2. Both models support the propagation of nonlinear waves of excitation and exhibit circular and spherical target patterns, linear and planar waves and spiral and scroll wave patterns in two and three dimensions respectively. The Fenton-Karma model features steep upstroke of the action potential and realistic restitution behavior. The Aliev-Panfilov model features restitution behavior qualitatively and was previously reported to be used together with soft-tissue elastic modeling in multi-physical simulations of heart tissue with mechano-electric coupling. ${ }^{106}$

Both models were solved computationally using finite differences numerical integration schemes with forward Euler time stepping methods. Excitation and refractory variables $u$ and $v$ of equations 2.1.13-2.1.14 and $u, v$ and $w$ of equations 2.1.17-2.1.22 respectively were computed in discrete timesteps $\Delta t$ on two- and three-dimensional simulation domains with a regular grid geometry with grid distances $\Delta x=\Delta y=\Delta z$ in $e_{x^{-}}, e_{y^{-}}$and $e_{z}$-directions respectively. In three-dimensional domains, the variables were updated sequentially over the indices $i, j, k$ in each time step:

$$
\begin{aligned}
u_{i j k}^{\tau} & :=u\left(\Pi_{e}(i, j, k), \tau \Delta t\right) \\
v_{i j k}^{\tau} & :=v\left(\Pi_{e}(i, j, k), \tau \Delta t\right) \\
w_{i j k}^{\tau} & :=w\left(\Pi_{e}(i, j, k), \tau \Delta t\right)
\end{aligned}
$$

to obtain the variable $u_{i j k}^{\tau}, v_{i j k}^{\tau}$ or $w_{i j k}^{\tau}$ respectively. The position function $\Pi_{e}$ that defines the position of each element of the electrical simulation grid is given by:

$$
\Pi_{e}(i, j, k)=\left(\frac{(1+2 i) \Delta x-L_{x}}{2}, \frac{(1+2 i) \Delta y-L_{y}}{2}, \frac{(1+2 i) \Delta z-L_{z}}{2}\right)
$$

with $0 \leq i \leq N_{x}-1,0 \leq j \leq N_{y}-1,0 \leq k \leq N_{z}-1$ and $N_{x}=L_{x} / \Delta x, N_{y}=L_{y} / \Delta y, N_{z}=$ $L_{z} / \Delta z$ and $0 \leq \tau \leq T / \Delta t$ so that the simulation area is given by $\left[-L_{x} / 2, L_{x} / 2\right] \times\left[-L_{y} / 2, L_{y} / 2\right] \times$ $\left[-L_{z} / 2, L_{z} / 2\right]$ as described previously. ${ }^{172}$ The grid points are centered at $\Delta x \times \Delta y \times \Delta z$. The distances $\Delta x, \Delta y$ and $\Delta z$ determine the behavior of the diffusion and can be scaled using a diffusion constant. The overall integration time was given by $T$. The forward Euler integration is given for the 
variables respectively by:

$$
\begin{aligned}
u_{i j k}^{\tau+1} & =u_{i j k}^{\tau}+\Delta t \cdot f\left(u_{i j k}^{\tau}\right)+\Delta t \cdot L_{i j k}^{\tau} \\
v_{i j k}^{\tau+1} & =v_{i j k}^{\tau+1}+\Delta t \cdot g\left(v_{i j k}^{\tau}\right) \\
w_{i j k}^{\tau+1} & =w_{i j k}^{\tau+1}+\Delta t \cdot h\left(w_{i j k}^{\tau}\right)
\end{aligned}
$$

with $L_{i j k}^{\tau} \approx\left[\nabla^{2} u\right]_{i j k}^{\tau}$ being the Laplacian operator, and $\left[\nabla^{2} u\right]_{i j k}^{\tau}=L_{i j k}^{\tau}+\left(\mathcal{O} \Delta x^{2}\right)$ being its order of magnitude. The dynamic variables $u$ and $v$ or $u, v$ and $w$ respectively are normalized dynamic variables respectively corresponding to the local excitation or refractoriness state of the tissue. The functions $f, g$ and $h$ The parameters used in this work for equations 2.1.13-2.1.14 are given in sections 5.2 and 5.3.1. The parameters used in this work for equations 2.1.17-2.1.22 are shown in table B.2.

\subsubsection{Diffusion}

The diffusive spread of electrical excitation through the multi-cellular cardiac substrate was simulated using Laplacian operators $\mathrm{L}$, which act on neighbouring nodes of the simulation grid. In three spatial dimensions, the Laplacian operator $L_{i j k}$ is given by a third order tensor or $3 \times 3 \times 3$ matrix with 27 components and indices $i, j, k$ :

$$
\begin{aligned}
L_{i j, k=2} & =\left(\begin{array}{ccc}
3 & 14 & 3 \\
14 & -128 & 14 \\
3 & 14 & 3
\end{array}\right) \\
L_{i j, k=1,3} & =\left(\begin{array}{ccc}
1 & 3 & 1 \\
3 & 14 & 3 \\
1 & 3 & 1
\end{array}\right)
\end{aligned}
$$

In two spatial dimensions, the Laplacian operator $L_{i j}$ is given by a second order tensor or $3 \times 3$ matrix with 9 components and indices $i, j$ :

$$
L_{i j}=\left(\begin{array}{ccc}
1 & 4 & 1 \\
4 & -20 & 4 \\
1 & 4 & 1
\end{array}\right)
$$

Both Laplacian operators allow isotropic diffusive spread of excitation during integration of the system.

\section{Arbitrary Geometries}

The above described spatial setup and the numerical implementation to simulate diffusive spread allows to simulate electrical wave activity in bulk-shaped geometries only. To be able to simulate 
electrical wave activity in arbitrarily-shaped domains, the phase-field method ${ }^{58}$ was used. Finite differences schemes lead to numerical instabilities and artifacts if integrated along the boundaries of arbitrarily shaped simulation domains and typically the finite-element method is used to perform computations on arbitrarily shaped simulation domains. However, the phase field method allows to approximate curved geometries using smoothing functions, offering in the same time the computational efficiency of finite differences schemes. The implementation of the phase-field method was done by P. Bittihn ${ }^{225}$ and provided for use within an open-source computational framework called MediaSim. ${ }^{225}$ The incorporation of the here described computational model into the above mentioned framework was done by S. Stein in his Master's thesis project, ${ }^{232}$ see also section 4.4.

\subsubsection{Initial Conditions}

To induce spiral and scroll wave patterns, as well as planar or spherical target patterns, the medium had to be prepared accordingly. Circular or spherical waves can be initiated, depending on the parametsr, by setting one or multiple neighbouring units above threshold. Linear or planar waves can be initiated by setting a line or a plane above on the boundaries of the medium threshold from. Spiral waves in two-dimensional domains using the Aliev-Panfilov model were induced by setting the excitatory variable $u$ of units along a straight line from the center of the medium to the center of one of the edges of the medium to $u_{i j}^{0}=1$ with $i=\left(N_{x}-1\right) / 2$ and $j=1, \ldots,\left(N_{y}-1\right) / 2$ and $u_{i j}^{0}=0$ otherwise and a parralel line right next to this line $v_{i j}^{0}=1$ with $i=\left(N_{x}-1\right) / 2-1$ and $j=1, \ldots,\left(N_{y}-1\right) / 2$ and $v_{i j}^{0}=0$ otherwise, see also figure 1.1. As a consequence, the medium was stimulated along the resulting line and excitation would start to spread away towards one side from the line and start to spiral around the center of the medium, see also figure 5.6 in chapter 5. Scroll waves can be initiated accordingly using the Aliev-Panfilov model. Using the Fenton-Karma model, spiral and scroll waves can be initiated by initiating a linear or planar wave and resetting one half of the wave at the desried point of reentry.

\subsection{Elasto-Mechanical Model}

The soft-tissue elasto-mechanics of cardiac tissue were simulated employing a dicrete elastic model, see section 4.2.1. The model allows to simulate elastic deformations of two- and three-dimensional continuum bodies, whith the deformations being possibly finite or also nonlinear. Also, deformations can be simulated to be anisotropic to capture the highly anisotropic elastic behavior of the cardiac muscle. Numerical integration schemes and computation times are comparable to the finite differences approach used to simulate electrical activity described in the previous section. The computational approach for simulation of actively contracting myocardial tissue is presented in section 4.3 after the setup of the numerical scheme for the simulation of the passive elasticity was described here. Passive elasticity refers to the passive elastic behavior in the absence of excitation-induced active stress.

\subsubsection{Simulation of Elasticity using Discrete Particle System}

Elastic behavior was simulated employing a discrete particle system with elastic particle-interactions in between particles. The elastic particle-interactions preserve the topology of the particle system and introduce a soft-tissue-like behavior, see also section 2.2.5 in chapter 2. The here presented approach is comparable to mass-spring damper systems, however, the setup of the particle lattice structure, 
force interactions in between particles and handling of the distribution of forces through the system are very different from conventional mass-spring systems.

Deforming elastic continuous bodies were approximated using a set of discrete particles $P^{(i)}$ distributed evenly at locations $\vec{x}_{i} \in \mathbb{R}^{3}$, with the space or volume $\Omega$ occupied by all particles $\vec{x}_{i} \in \Omega$ making up the continuum body. Each particle's motion is governed by the Newtonian equation of motion:

$$
m_{i} \ddot{\vec{x}}_{i}=\sum_{j} \mathbf{f}_{i j}(\vec{x})
$$

where $\mathbf{f}_{i j}$ are the $j$ forces which act on the particle $i$. Each particle experiences forces by its surrounding, neighbouring particles and may additionally experience globally acting forces. Generally, the forcing behavior in between particles may take on any form and does not necessarily have to be directed along the direction with the shortest euclidean distance in between two particles, see following section. Two- or three-dimensional simulation domains were discretized into square-shaped area or hexaedral volume cells respectively and domains were restricted to be rectangular or cubic-shaped bulk domains unless stated otherwise, see section 4.4. To simulate cubic-shaped bulk media, particles were positioned in space on a three-dimensional regular lattice with positions ordered by three indices $i, j$ and $k$ :

$$
\vec{x}_{i j k}=\Pi_{m}(i, j, k)
$$

with the position function $\Pi_{m}$ being given by:

$$
\Pi_{m}(i, j, k)=\left(\frac{(1+2 i) \Delta x-L_{x}}{2}, \frac{(1+2 i) \Delta y-L_{y}}{2}, \frac{(1+2 i) \Delta z-L_{z}}{2}\right)
$$

with $0 \leq i \leq N_{x}, 0 \leq j \leq N_{y}, 0 \leq k \leq N_{z}$ and $N_{x}=L_{x} / \Delta x, N_{y}=L_{y} / \Delta y, N_{z}=L_{z} / \Delta z$ and $0 \leq \tau \leq T / \Delta t$ so that the simulation area is given by $\left[-L_{x} / 2, L_{x} / 2\right] \times\left[-L_{y} / 2, L_{y} / 2\right] \times$ $\left[-L_{z} / 2, L_{z} / 2\right]$ as described previously. ${ }^{172}$ Note, that the position function $\Pi_{m}$ is different from the position function $\Pi_{e}$ in the previous section. The overall number of particles $N$ is given by $N=N_{x} N_{y} N_{z}$.

\section{Muscle Fiber Anisotropy Control}

The elastic model allows to simulate muscle fiber anisotropy following a numeric scheme first reported by Bourguignon et al. ${ }^{67}$ Muscle fiber anisotropy is one of the most important elastic features of cardiac tissue as the muscle is organized in muscle fiber bundles and sheets that are stacked orthotropically, see chapter 1. Anisotropic elastic behavior is inherent in particle system models, included in the discrete formulation of the system per definition. The anisotropy is introduced by the lattice structure that defines the spatial organization of particles. Figure 4.1(a) shows a conventional 
layout of a mass-spring damper system. Every node of the grid is connected to its 4 nearest neighbours via springs. As a result, the regular lattice structure introduces undesired anisotropy along the $e_{x}$ - and $e_{y}$-directions of the particle system. Also other springs such as diagonal or second-nearest neighbour springs can be introduced, each of which introduce their own anisotropic behavior. Conventional mass-spring systems can be adjusted to behave like so-called Seth-materials, ${ }^{, ?}$, which are isotropic in the limit of the continuum. However, they do not offer the possibility to define arbitrary preferred orientations of anisotropy. The layout of the particle system used in this work allows to control the anisotropy ${ }^{67}$ by introducing springs that can be rotated freely to point in any arbitrary direction. Particles are organized as before on a regular lattice structure, as indicated by grey dots in figure 4.1(b). However, the springs are not laid out on a regular lattice and do not connect particles directly with each other. Instead, each volume cell that is given by 4 or 8 neighbouring particles, in two or three dimensions respectively and indicated by gray edges in figure 4.1(b), contains a set of orthogonal springs, again with 4 or 6 springs in two or three dimensions respectively. The set of springs manages the elastic deformation of the cell. The origins of the sets of orthogonal springs, indicated by black dots in figure 4.1(b), are located at the barycenter of each cell, that is the average position of its surrounding particles, see below. Each individual spring of the set connects to the barycenter as well as to one point $\vec{x}_{c}$ on the surface of the hexaedral cell, or to one point on the cell's edges in two dimensions respectively, as indicated by the red dots in figure 4.1(b). In order to specify the anisotropic behavior of the elasticity, an initial material configuration of the springs with specific orientations needs to be chosen in the undeformed reference configuration $\chi_{0}$ of the system before the start of the simulation, see also below. Then, the coordinates of the points $\vec{x}_{c}$ to which the springs attach are derived using ray-tracing techniques? and stored as bilinear coefficients to be used to compute the locations of the connecting points $\vec{x}_{c}$ in the deformed configuration $\chi_{t}$ at any later point in time. Every cell shares edges or surfaces with its neighbouring cells and the forces between particles are transmitted from cell to cell over these interfaces. Neighbouring particles experience forces from the neighbouring cell's interface according to a weighted contribution determined by the bilinear coefficients. To each set of orthogonal springs belongs a set of angular springs which preserves the perpendicular angles between the springs of the set. The system can additionaly include other regular springs as indicated in figure 4.1(a). In this work, both approaches, a conventional mass-spring damper system and the here presented scheme, were implemented within the same simulation framework in parallel and could selectively be switched on and off. Note that in figure 4.1(a) particles are indicated by black dots, whereas in figure 4.1(b) particles are indicated by gray dots to facilitate viewing. In particular, in the scheme shown in (b) one unit of the elastic system consists of a cell with its surrounding particles, whereas in the conventional mass-spring damper system shown in (a) one unit consists of a particle with its surrounding springs. The above described modeling approach was reported previously to be used for modelig of cardiac tissue, ${ }^{132,201,214}$ however, in slightly different modeling approaches as described here.

In three dimensions, each cell is represented by a hexaedral cell with 8 vertices, 12 edges and 6 surfaces. In the undeformed reference configuration $\chi_{0}$ the hexaedral cell is cubic-shaped with edge lengths $\Delta x=\Delta y=\Delta z=1$. The origin of the set of orthogonal springs with 6 principle orientations $\mathbf{e}_{1}, \mathbf{e}_{2}, \mathbf{e}_{3}, \mathbf{e}_{4}=-\mathbf{e}_{1}, \mathbf{e}_{5}=-\mathbf{e}_{2}, \mathbf{e}_{6}=-\mathbf{e}_{3}$ and $\mathbf{e}_{1} \perp \mathbf{e}_{2}$ and $\mathbf{e}_{2} \perp \mathbf{e}_{3}$ is positioned at the barycenter given by the average position of the particles or vertices defining the cell:

$$
\vec{x}_{b}=\frac{1}{8} \sum_{p}^{8} \vec{x}_{p}
$$




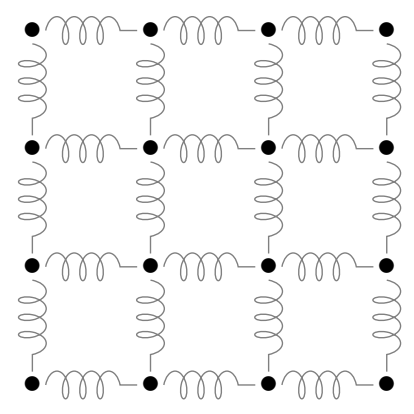

(a)

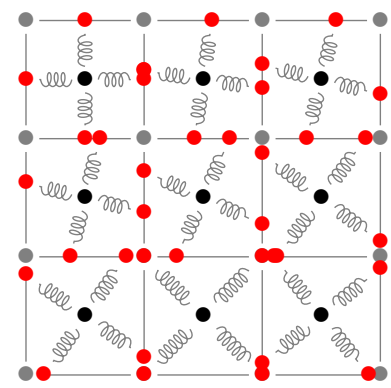

(b)

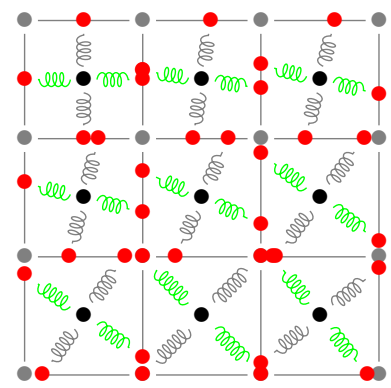

(c)

Figure 4.1: Particle systems for elasticity modeling: (a) conventional mass-spring damper system with regular lattice structure and regular and rigid spring configuration, (b) elastic particle system composed of orthogonal sets of springs that are adjustable and can be rotated for anisotropy control, springs are attached to surfaces or edges (red points) of cells from where forces are transmitted to the next cell (c) same scheme as shown in (b) with active springs (green), which shorten upon excitation (green dot at barycenter in upper left cell) and lead effectively to a contraction of the cell in electromechanically coupled simulations, see section 4.3.

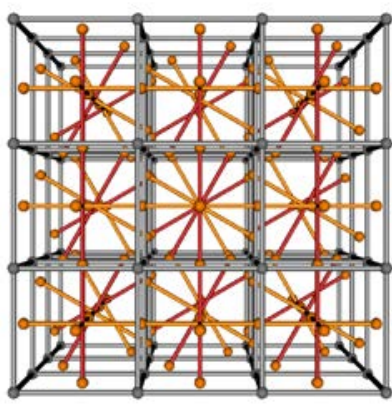

(a)

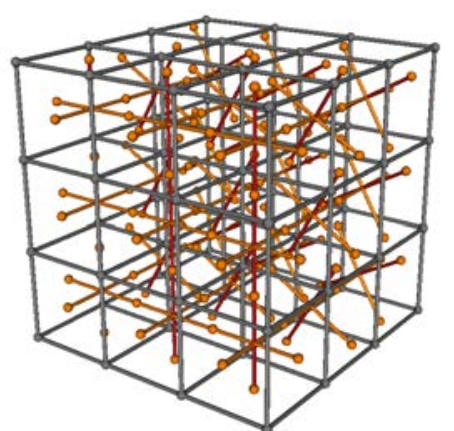

(b)

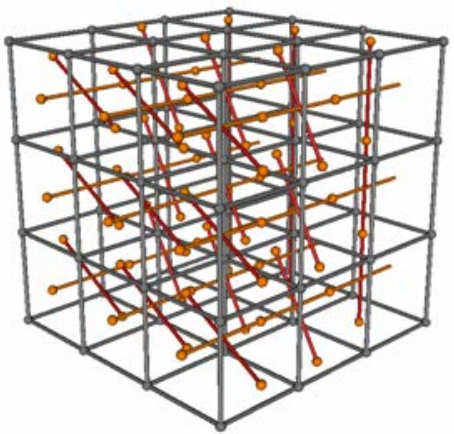

(c)

Figure 4.2: Elastic particle system with anisotropy control: ${ }^{67}$ (a) cubic-shaped medium constructed from $4 \times$ $4 \times 4$ particles or $3 \times 3 \times 3$ hexaedral volume cells with 8 vertices, 12 edges and 6 faces each, indicated by gray points and edges. Gray edges are shown for visualization purposes only and do not represent physical forcing elements of the elastic particle system. Each hexaedral cell contains a set of 6 orthogonal springs, where 3 pairs of springs each consist of 2 antiparralel springs which point from the barycenter of the cell outwards and attach to the surface of the cell, leaving 3 principal orientations per hexaedral cell. Each principal direction may be understood to indicate the muscle fiber-, sheet- and sheet-normal directions in the tissue. The sets of springs can be rotated to obtain different muscle fiber configurations. 
with the positions $\vec{x}_{p}$ of the $p=1, \ldots, 8$ particles such that in the undeformed configuration $\chi_{0}$ the barycenter of particle $P^{(i)}$ is given by $\vec{x}_{b}^{i}=\vec{x}^{i}+(\Delta x / 2, \Delta y / 2, \Delta z / 2)^{t}$. One of the spring orientations is chosen to be the primary orientation $\mathbf{e}_{1}$ and set to point from the barycenter into an arbitrary direction given in spherical coordinates $(r=1, \theta, \varphi)$. The other two orthogonal orientations $\mathbf{e}_{2}$, $\mathbf{e}_{3}$ are computed using the cross product of the primary orientation $\mathbf{e}_{1}$ with an arbitrary orientation $\mathbf{e}_{a}$, such that for instance $\mathbf{e}_{2}=\left(\mathbf{e}_{1} \times \mathbf{e}_{a}\right) /\left(\left|\mathbf{e}_{1} \times \mathbf{e}_{a}\right|\right)$. The antiparallel orientations $\mathbf{e}_{4}, \mathbf{e}_{5}$ and $\mathbf{e}_{6}$ are computed accordingly. The 6 intersection points $\vec{x}_{c}$ of the 6 principle orientations of the springs with the surface of the hexaedral cell are obtained using ray-tracing techniques and are consequently described as a bilinear interpolation of the 4 corresponding vertices of that face that is being intersected. For instance, if the face is defined by the 4 vertices $\vec{x}_{1}, \vec{x}_{2}, \vec{x}_{3}, \vec{x}_{4}$ the coefficients define the intersection point $\vec{x}_{c}$ as follows: ${ }^{67}$

$$
\vec{x}_{c}=\xi \eta \vec{x}_{1}+(1-\xi) \eta \vec{x}_{2}+(1-\eta)(1-\xi) \vec{x}_{3}+(1-\eta) \xi \vec{x}_{4}
$$

where $\xi$ and $\eta$ are the bilinear interpolation coefficients. In the deforming configuration $\chi_{t}$, the springs connect the barycenter $\vec{x}_{b}$ with the respective intersection point $\vec{x}_{c}$ given by the actual position of the 4 vertices defining the corresponding face and the corresponding bilinear interpolation coefficients $\xi$ and $\eta$, which were derived in the undeformed configuration $\chi_{0}$. The spring forces along the six principle axis orientations act accordingly between the corresponding intersection point $\vec{x}_{c}$ with $c=1, \ldots, 6$ and the barycenter $\vec{x}_{b}$, see also section 2.2 .5 in chapter 2 , as follows:

$$
\mathbf{f}_{c}(x)=-k_{s}\left(\left|\vec{x}_{c}-\vec{x}_{b}\right|-\left|\mathbf{l}_{c, 0}\right|\right) \cdot \mathbf{e}_{k}
$$

where $k_{s}$ is the stiffness constant of the spring, $\vec{x}_{c}$ and $\vec{x}_{b}$ are the positions of the barycenter and the intersection point in the deformed configuration, $\mathbf{l}_{c, 0}$ ist the rest length of the spring in the undeformed configuration and $\mathbf{e}_{k}$ is the respective orthonormal principle orientation of the spring in the deformed configuration. Angular forces between the three principal axises are introduced by angular or torsion springs. Their rest lengths are defined as $\left|l_{\phi, 0}\right|=\cos \left(\mathbf{e}_{1} \cdot \mathbf{e}_{2}\right)$ in the undeformed configuration $\chi_{0}$

$$
\begin{aligned}
\mathbf{f}_{2 k-1}(\phi) & =-k_{s}\left(\mathbf{e}_{1} \cdot \mathbf{e}_{\perp}-\left|l_{\phi, 0}\right|\right) \cdot \mathbf{e}_{\phi} \\
& =-\mathbf{f}_{2 k}(\phi)
\end{aligned}
$$

Figure 4.2 shows several examples of spring configurations inside a three-dimensional cubic-shaped medium constructed from $4 \times 4 \times 4$ particles or $3 \times 3 \times 3$ hexaedral volume cells correspondingly. The 8 vertices and 12 egdes of each cell are indicated as gray dots and lines. Note, that gray edges are shown for visualization purposes only and do not correspond to physical forcing elements of the elastic particle system. Each hexaedral cell has 6 faces and contains an orthogonal set of springs at its barycenter, indicated by yellow lines. The configuration of spring orientations can be specified to be random, linearly uniform transverse or chirally rotating, for instance. Each principal orientation of the springs can be understood to indicate the muscle fiber-, muscle sheet- and muscle sheet-normal directions in the tissue respectively.

\subsubsection{Verlet Integration}

To compute the dynamical behavior of the elastic particle system, the trajectory $\vec{x}(t)$ of every particle $P^{(i)}$ of the system was computed using the Verlet-algorithm. The equation of motion for one 
particle's position is given by:

$$
\begin{aligned}
\vec{x}_{n+1} & =\vec{x}_{n}+\vec{v}_{n} \Delta t+\frac{1}{2} \vec{a}_{n} \Delta t^{2} \\
& =\vec{x}_{n}+\frac{\vec{x}_{n+1}-\vec{x}_{n-1}}{2 \Delta t} \Delta t+\frac{1}{2 m} \vec{f}_{n}(\vec{x}, \vec{v}) \Delta t^{2}
\end{aligned}
$$

where $\vec{x}_{n+1}$ is the new position of the particle and $\vec{x}_{n}, \overrightarrow{v_{n}}$ and $\overrightarrow{a_{n}}$ are the positions, velocities and accelerations of the particle in the current timestep and $\vec{x}_{n-1}$ and $\vec{x}_{n+1}$ are the positions in the previous and the future time step. The Verlet-method employs specific cubic properties of the equation of motion to provide a very simple, yet very stable scheme for numerical integration. In one spatial dimension, the Verlet method is based on two third-order Taylor expansions of a position $x(t)$, one forward in time and one backwards in time:

$$
\begin{aligned}
& x(t+h)=x(t)+\dot{x}(t) h+\frac{1}{2} \ddot{x}(t) h^{2}+\frac{1}{6} x^{(3)}(t) h^{3}+\mathcal{O}\left(h^{4}\right) \\
& x(t-h)=x(t)-\dot{x}(t) h+\frac{1}{2} \ddot{x}(t) h^{2}-\frac{1}{6} x^{(3)}(t) h^{3}+\mathcal{O}\left(h^{4}\right)
\end{aligned}
$$

Adding the equations and isolating for $x(t+h)$ yields:

$$
x(t+h)=2 x(t)-x(t-h)+\ddot{x}(t) h^{2}+\mathcal{O}\left(h^{4}\right)
$$

Accordingly, the above equation 4.2 .7 reduces to:

$$
\vec{x}_{n+1}=2 \vec{x}_{n}-\vec{x}_{n-1}+\frac{1}{m} \vec{f}_{n}(\vec{x}, \vec{v}) \Delta t^{2}
$$

such that the term $\vec{x}_{n+1}$ in the velocity term of equation 4.2 .7 , which would have required knowledge about future time steps, cancels out and the only required input is the actual and previous position of the particle $P^{(i)}$ and the forces acting on it. The force term $\vec{f}(\vec{x}, \vec{v})$ may include frictional forces which lead to a dissipation of energy:

$$
\vec{f}_{n}(\vec{x}, \vec{v})=\tilde{\vec{f}}_{n}-\gamma \vec{v}_{n}
$$

where $\gamma$ is the friction constant. Including frictional forces affecting all velocities, equation 4.2.11 alters to:

$$
\vec{x}_{n+1}=\frac{2 \vec{x}_{n}-\vec{x}_{n-1}\left(1-\frac{\gamma}{2 m} \Delta t\right)+\frac{1}{m} \vec{f}_{n} \Delta t^{2}}{1+\frac{\gamma}{2 m} \Delta t}
$$




\subsection{Actively Contracting Myocardial Tissue}

Actively contracting myocardial muscle tissue was simulated by coupling the two computational models described in the two previous sections 4.1 and 4.2 in one multi-physical simulation, such that nonlinear waves of electrical excitation would trigger contractions of the elastic medium. More specifically, electromechanical coupling between electrical and mechanical parts of the simulation was implemented only in forward direction, compare with section 2.3 in chapter 2 , with the excitation causing the medium to deform but without the mechanics affecting the behavior of the electrical activity. The effect of stretch-activated channels or altered diffusion properties due to mechanical deformations did not seem relevant with regard to the scientific questions raised and investigated in this thesis. Furthermore, electromechanical coupling consisting of forward and backward coupling was considered to lead to unnecessary overcomplication of the problem.

Contractions are generated by active springs introduced to the elastic particle system described in section 4.2.1. Figures 4.1(c) and 4.2 depict the active springs to be part of the set of orthogonal springs contained within every cell of the particle system. The active stress dynamic variable $T_{a}$ that is coupled to the excitation variable $u$ of equations 2.1 .13 or 2.1.17, see also section 2.1.5 in chapter 2, modulates the rest lengths of the active springs such that springs shorten upon electrical excitation. This causes effectively a local contraction with particles being accelerated towards each other. Because active springs are introduced only along one of the three main principal orientations in each orthogonal set of springs, see figure 4.1(c), the resulting contractions are locally linearly transverse isotropic. The overall deformation can become highly anisotropic if the configuration of spring orientations is set up appropriately, see also figure 4.2. The active stress development was modeled using an additional partial differential equation, see equation 2.3.3, that couples the active stress to the excitation state of the system: ${ }^{106}$

$$
\frac{\partial T_{a}}{\partial t}=\varepsilon(u)\left(k_{T} u-T_{a}\right)
$$

where $T_{a}$ is the active stress dynamic variable and $k_{T}$ is a parameter regulating the strength of active stress build-up.

\subsection{Realistic Heart Modelling}

To study electromechanical wave activity on realistically heart-shaped simulation domains, the elastic computational model described in the previous section 4.3, which only allowed to simulate electromechanical wave activity inside bulk-shaped media, was modified and extended. Several modifications and enhancements had to be incorporated to allow simulations on realistically heart-shaped simulation domains. First, the simulation had to be able to handle in and outside of the simulation domain on a voxel-based simulation grid. Data read-in of arbitrarily shaped, possibly porous media had to be implemented with the goal to be able to read in segmented image data showing hearts and other heart tissue preparations imaged in experiments. To approximate curved boundaries during the discretization procedure of arbitrarily shaped simulation domains more accurately, the discretization procedure was changed from using hexaedral volume elements to tetrahedral volume elements. ${ }^{67,201,214,232}$ As a result, every node of the electrical simulation grid was associated with 4 tetrahedras instead of 
with one hexaedra, compare also with figure 4.1(c). At the same time, the number of particles of the system stayed the same. Lastly, the electrical part of the simulation had to be able to perform computations on arbitrarily shaped simulation domains as well, see also section 4.1.1. Therefore, the elastic model was integrated into the simulation framework MediaSim, a computational framework developed by P. Bittihn ${ }^{225}$ and others, which allows to perform these kinds of simulations on arbitrarily shaped domains. This work, the incorporation of tetrahedral discretization, the implementation of data read-in of real heart geometries to setup heart-shaped elastic simulation domains and the consolidation of the elastic model with the simulation framework MediaSim was conducted by S. Stein during his Master's thesis project. ${ }^{232}$

\subsubsection{Rabbit Heart Model}

A rabbit heart model data set was developed from experimental data. The data was acquired during the experiments described in chapter 6 within the scope of this thesis. The model was suppoed to be used with the computational framework described in the previous section. The simulations shown in figures 5.17 and 5.18 were computed on the developed model. The following steps were realized together with D. Hornung, S. Stein and E. Boccia.

\section{Volumetric Micro-CT Scans with Fiber Staining}

Formalin-fixed isolated rabbit hearts were scanned using computerized tomography to obtain volumetric scans of the hearts of dimensions $\sim 1500 \times 1500 \times 1500$ voxels. The resultant scans were supposed to reveal the geometrical structure of the anatomy of heart, including its chambers and ventricles, as well as the underlying muscle fiber orientation inside the ventricular walls. Hearts were washed and stained using iodine solution as described previously. ${ }^{224}$ Iodine is thought to pronounce diadic clefts as well as muscle fiber anisotropy. Hearts were scanned using a computer tomography imaging system with micrometer spatial resolution, see figure 3.2. Imaging was performed together with D. Hornung.

\section{Shape Reconstruction from Image Segmentation and Tetrahedral Discretization}

The obtained computerized tomography scans were reduced in size to about $200 \times 200 \times 200$ voxels. To extract the geometry of the heart, the volumetric scan was manually segmented using image segmentation software. The resulting binary volume was used to reconstruct a heart-shaped elastic particle system with tetrahedral discretization. Every voxel was represented by $0-4$ tetrahedra, depending on its position within the tissue volume and whether it showed tissue or no tissue or was located at the boundaries of the tissue within the simulation domain. The latter step was implemented by S. Stein during his Master's thesis project. ${ }^{232}$

\section{Muscle Fiber Field Reconstruction}

The muscle fiber field was extracted from the three-dimensional grayvalue pattern of the voxels of the computerized tomography scans. The scans were analyzed using volumetric kernels that computed the similarity of the surrounding tissue with respect to the selected pixel to obtain a three-dimensional profile, the local structure tensor $\mathcal{F}$, which was found to exhibit anisotropy that, if obtained for every voxel inside the volume, provided the picture shown in figure 4.4. This work was done in collaboration with E. Boccia. 


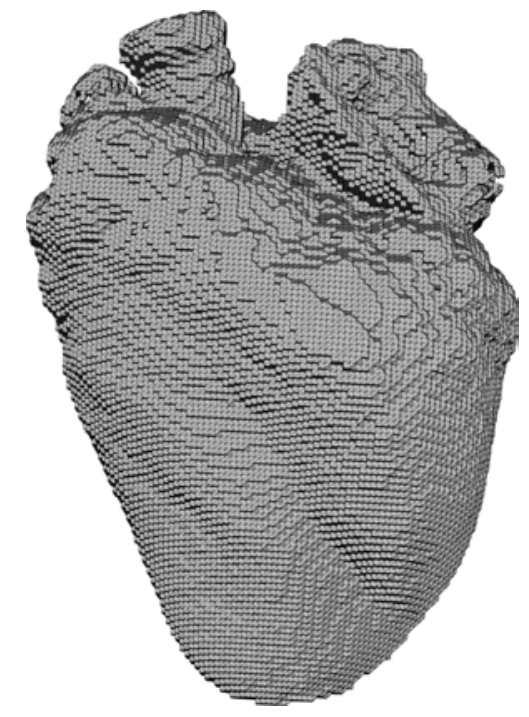

Figure 4.3: Reconstructed and discretized rabbit heart geometry: shape reconstruction using manual image segmentation of $\mu$-CT data showing rabbit heart, resulting segmented volume data was used to reconstruct the heart shape by tertrahedral volume elements, figures provided by S. Stein ${ }^{232}$
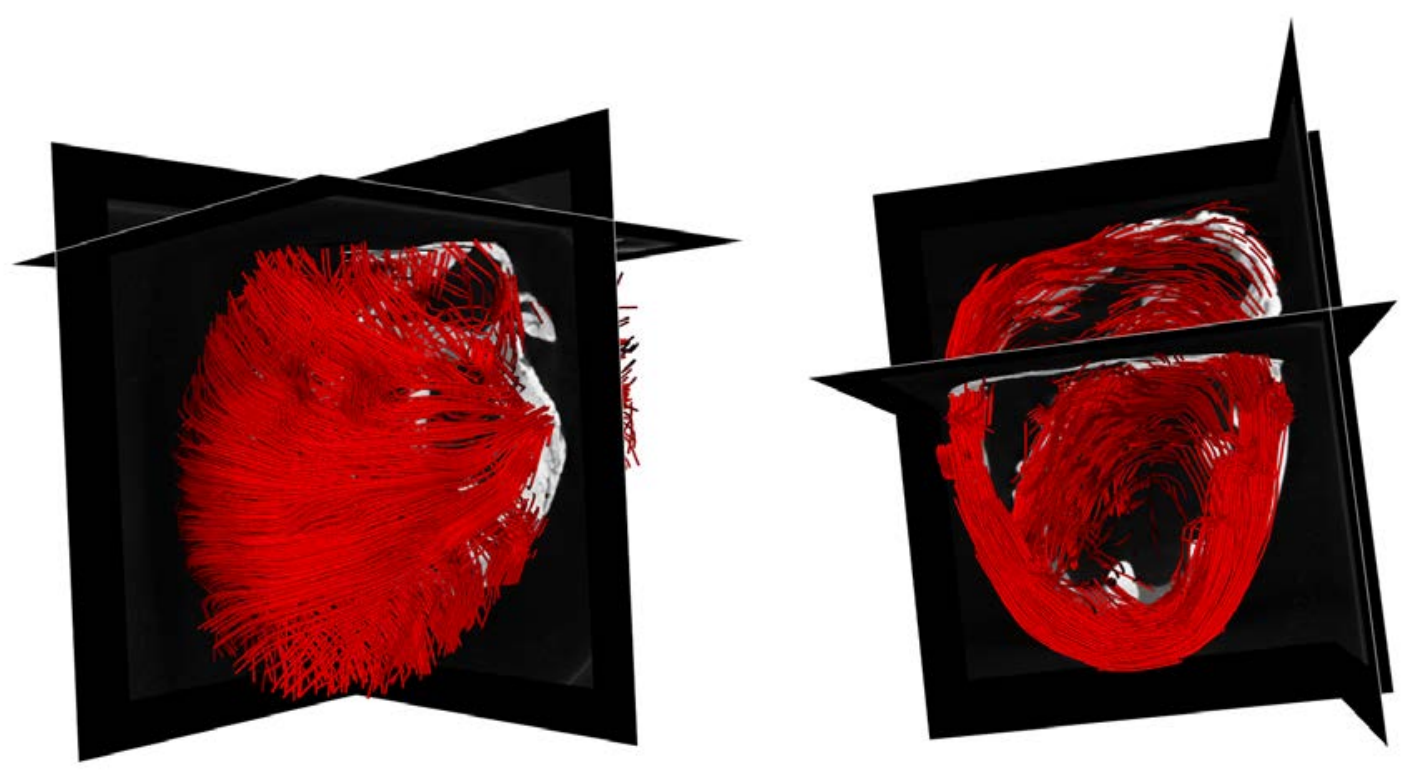

Figure 4.4: Muscle fiber field reconstruction from experimental data of a rabbit heart, visualized using streamlines: orientation field derived from anisotropic local structure tensor $\mathcal{F}$ indicated by red streamlines. Local structure tensor $\mathcal{F}$ derived by analyzing three-dimensional grayvalue data obtained from volumetric micro-CT scan of formalin-fixed rabbit heart. Streamlines following approximately radial orientations of actual muscle fiber orientation field in the ventricles. Data retrieved from data set as shown above. Data analysis performed together with E. Boccia. 
Chapter 5. Electromechancial Wave Pattern Reconstruction

\section{Chapter 5}

\section{Electromechancial Wave Pattern Reconstruction}

Electromechanical wave patterns are coupled electrical wave- and mechanical deformation patterns in the heart muscle. Due to the excitation-contraction coupling mechanism, electrical excitation triggers contractions of cardiac muscle cells, which leads to a deformation of the cardiac muscle, see chapter 1 . The electrical excitation spreads diffusively through the myocardial muscle and is typically described to retain a wave-like character, see chapter 1 and 2. Mathematically, the wave-like behavior is modeled employing nonlinear waves of excitation. ${ }^{51,58,60}$ The coupled mechanical behavior, however, retains a different nature, as it involves deformation mechanics that requires mathematical descriptions involving tensor mathematics. Attributing a wave-like behavior to the mechanical behavior of the heart ${ }^{199,205,206,215}$ becomes possible when understanding that within the tissue are active processes at work, that are wave of contractile activity. The heart contracts, and the onset of contraction is accompanied by elastic strain or contraction waves. In this work, electromechanical waves are understood to be composed of spreading waves of electrical excitation, that are immediately followed by a wave-like spreading of deformation, with elastic strain waves emerging in accordance with the spreading excitation. The wave-like character of the mechanical activity is accordingly implied by the fact that the spreading waves of excitation lead to a spreading onset of deformation.

In this chapter, electromechanical wave patterns are studied in-silico to determine the fundamental relationship between the electrical and the according mechanical pattern during coupled electromechanical wave activity in elastic excitable media. Understanding electromechanical wave patterns is crucial for several reasons. ${ }^{106,148}$ One of the more important reasons is that understanding of the mechanical behavior of the heart during normal as well as arrhythmic cardiac activity opens the way for new non-invasive imaging techniques that could potentially be used to diagnose and study cardiac disease. ${ }^{192,205,206,215}$ Exploring the feasibility of such imaging techniques is one of the main aims of this thesis, see section 1.5. The deformation of the cardiac muscle can be imaged non-invasively using tomographic imaging techniques such as ultrasound. Given that the relationship between mechanical and electrical activity of the cardiac muscle is sufficiently well known, one may inversely image the electrical activity by reconstructing the electrical pattern that led to the according captured mechanical deformation. ${ }^{192}$ Hence, studying electromechanical wave activity in-silico is one of the first necessary steps to come to a better understanding of the fundamental dynamics that underlie the deforming, actively contracting heart muscle. This chapter aims to investigate the relationship between electrical and mechanical activity by analyzing the correlation of the two time-varying spatial- 
temporal electrical and mechanical patterns.

Other reasons to study electromechanical wave activity are motivated by more fundamental sientific questions, as it is established that a series of phenomena related to cardiac arrhythmia stem from electromechanical activity of the heart. For instance, it is known that excessive mechanical shocks can trigger arrhythmias ${ }^{79}$ or that spontaneous arrhythmias can be induced mechanically in acute regional ischemia. ${ }^{191}$ Moreover, as electrical and mechanical activity influence each other, the overall activity may lead to electromechanical feedback, ${ }^{106}$ a dynamical state which is suspected to be able to both promote but also inhibit arrhythmias. In addition to experimental studies, in-silico studies postulate new behaviors of the cardiac substrate as mechanical activity is taken into account. For instance, it was demonstrated that electromechanical wave activity exhibits new mechanisms of spiral wave initiation ${ }^{208}$ and break-up ${ }^{223}$ and may exhibit self-organized pace-making ${ }^{144}$ and alter the unpinning behavior $^{211}$ of spiral and scroll waves from heterogeneities inside cardiac tissue. Many of these effects are insufficiently studied, because the field of electromechanical computational modeling is relatively young and correspondingly there are only few reported in-silico studies. ${ }^{106,144,148,208,211,223}$ The work presented in this chapter also provides novel concepts for the characterization of coupled electromechanical spiral and scroll wave dynamics to be used in future in-silico studies of electromechanical wave activity.

\subsection{Electromechanical Coupling and Wave Hypothesis}

The analysis presented in this as well as the following chapter is based on the assumption that both electrical and mechanical activity in the heart are immediately electromechanically coupled entities with excitation-contraction coupling manifesting in two closely related spatial-temporal patterns. As already stated in the introduction of this chapter, in this study, electromechanical waves are understood to be composed of spreading waves of electrical excitation, immediately followed by a wavelike spreading of deformation, with elastic strain waves emerging in accordance with the spreading excitation. This section introduces the fundamental assumptions that were made in this thesis and explains the motivations that led to these assumptions, before conducting computer simulations and analyzing the experiments presented in chapter 6 .

The biophysical mechanisms leading to excitation-contraction coupling are based on an interdependence between transmembrane potential $V_{m}$ of the cell and intracellular Calcium $\left[\mathrm{Ca}^{2+}\right]_{i}$ release, where the depolarization of the transmembrane potential triggers the release of intracellular Calcium, which in turn fuels the contraction of the cardiomyocyte, see figure 1.6 in the introduction. On a cellular level, coupled electromechanical waves are, as they are passing through each cell, a composition of three subsequent waves, where an action potential (green curve) is followed by a calcium wave (black curve) and finally a wave-like onset and release of contraction (red curve). On the tissue level, however, this picture lacks to explain how the excitation-contraction coupling mechanism manifests inside an elastic continuum of cells, and accordingly needs to be extended, see figure 5.1. The contraction of the cardiac muscle is a continuum mechanical quantity that arises from an entirety of cells exerting contractile forces. As a result, the deformation of the cardiac muscle can become far more complicated than the wave-like behavior of the contraction of a single cell, as depicted by the red curve in figure 1.6 or 5.1, suggests. First, the contractile forces occur along distinct preferred orientations that are given by the muscle fiber configuration inside the muscle. Next, the synchrony and coherence of the contractions and resultant shape of the deformation depend on the underlying 


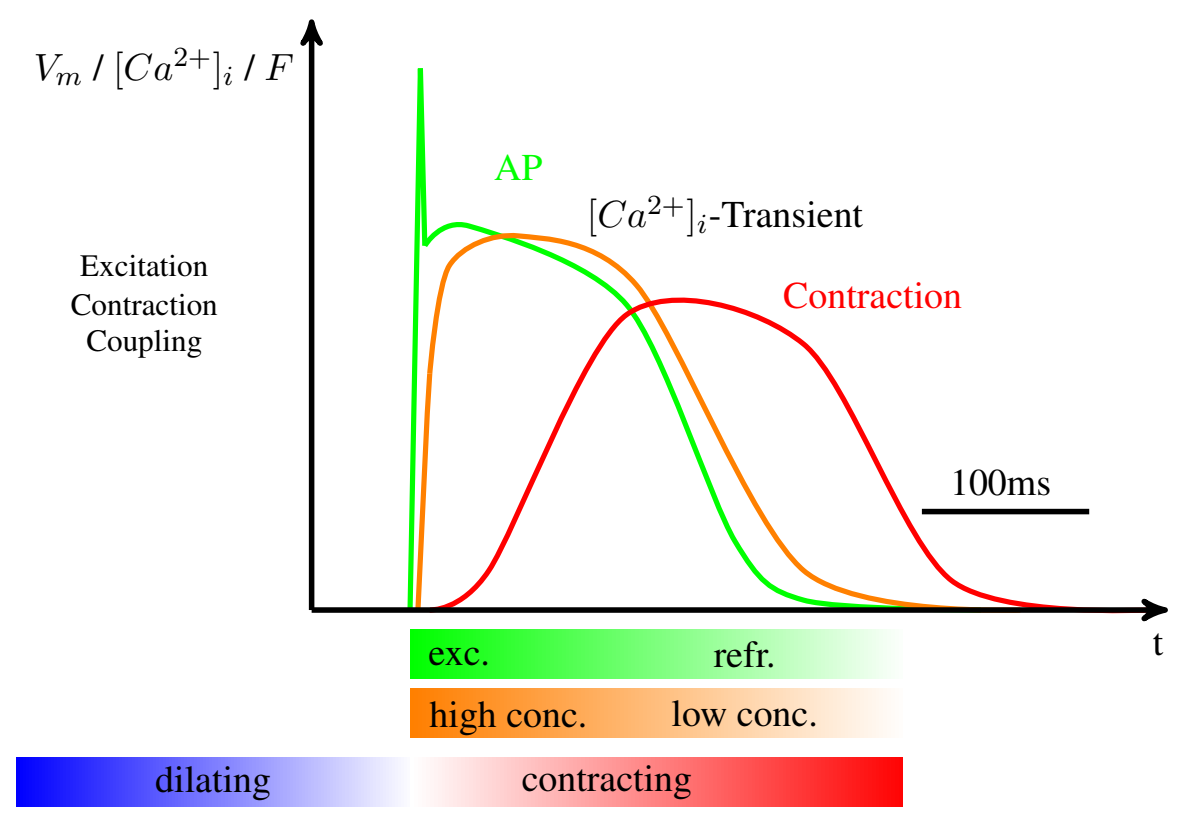

Figure 5.1: Macroscopic, continuum mechanical manifestation of excitation-contraction coupling mechanism in cardiac tissue: action potential (green curve) triggers release of intracellular calcium $\left[\mathrm{Ca}^{2+}\right]_{i}$ (orange curve), which in turn fuels contractions (red curve) of the cell. On the tissue level, the contractions of many cells lead to a global deformation of the cardiac muscle, which can be characterized to be composed of elastic strain waves caused by spreading onset of contraction. The elastic strain waves are composed of tensile and compressile strains and rates of strain or deformation respectively and can be derived from the Cauch-Green or Green-Lagrangian deformation tensors $\mathbf{C}$ or $\mathbf{E}$.

excitation pattern orchestrating the contractile activity of the cells. Long range elastic effects may superimpose the local contractile behavior. Also, the underlying calcium cycling and active stress build-up and conversion into myofibril contraction may be heterogeneous. Lastly, in addition to muscle fiber anisotropy, the overall structural organization and heterogeneous elasticity of the cardiac muscle, on various hierarchical levels, may cause a highly anisotropic and heterogeneous elastic behavior. These factors may severly constrain the applicability of the asumption postulated above.

To come to a better understanding of electromechanical wave propagation in cardiac tissue, it can be remunerative to modify and extent the elemental picture of excitation-contraction coupling, as it is depicted in figure 5.1. The figure is an adaptation from Bers ${ }^{83}$ see also figure 1.6 in the introduction, and shows the basic mechanisms associated with excitation contraction-coupling inside a single cardiomyocyte, but furthermore also shows how this excitation-contraction coupling mechanism may be understood to manifest inside an elastic continuum of cells. Here, it is postulated that excitationcontraction coupling, on the tissue level, leads to a characteristic dynamical behavior of the elasticity and that this behavior can be thought of to constitute the wave-like character of electromechanical wave activity, which can also be used to describe and measure electromechanical wave propagation in cardiac tissue. Every site in the continuum of cells experiences its surrounding via long range elastic effects. That is, contractile activity at a distance may cause a tensile deformation of a local piece of tissue elsewhere. Specifically, while electrical wave activity spreads through the cardiac 

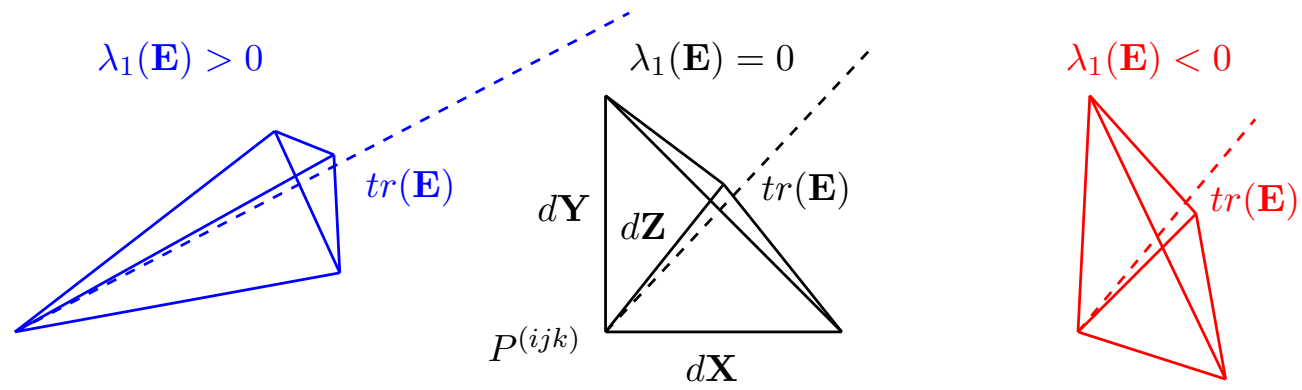

Figure 5.2: Deformation measures used for the visualization of two- or three-dimensional deformation patterns and elastic strain waves in elastic excitable media during coupled electromechanical wave activity: tensile and compressile deformation or rates of deformation derived from deformation tensors $\mathbf{E}$ or $\mathbf{C}$ of material volume element or material prism, see also section 2.2 in chapter 2.

muscle and causes locally parts of the tissue to contract, other parts of the tissue experience this activity by being pulled towards these sites that exert contractile forces. Now, if the electrical activity approaches a site, which may be the site depicted in figure 5.1, and causes also this site to actively contract, it is this very moment that reveals the trespassing of an electrical wave when monitoring the deformation state of the tissue. Shortly before the electrical wave front arrives, the tissue experiences a dilation or, if in an already contracted deformed state, is dilating. As the electrical wave front passes through, the tissue starts to contract and experiences a swift change from dilating to contracting rates of deformation, to become possibly contracted, if previously in a dilated state. This situation is depicted by the horizontal colorbars in the lower half in figure 5.1. The horizontal bar showing the blue-white-red color scheme indicates the deformation or rate of deformation of the local piece of tissue. The blue side of the bar corresponds to tissue lying in front of the approaching electrical wave front experiencing tensile strains or strain-rates. The red side of the bar corresponds to tissue lying inside the electrical or electromechanical wave experiencing compressile strains or strain-rates. The white part in between blue and red sides of the bar marks the transition from dilated or dilating tissue over a neutral or undeformed state to contracted or contracting tissue. This region coincides with the upstroke or front of the electrical wave that is also depicted by the green horizontal bar. In the following sections, this picture is used to depict the time-varying elastic deformation patterns that result in simulations of coupled electromechanical wave activity.

\subsubsection{Deformation Measures}

Mechanical deformation is a complex measure typically expressed in terms of deformation tensors of second order with $3 \times 3$ entries, see section 2.2 in chapter 2 . These tensors describe the local state of deformation of a material volume element or material prism as depicted in figure 5.2. To be able to visualize two- or three-dimensional tensor fields, which describe the full deformation state of a deforming continuum body, it is necessary to reduce ${ }^{72,81}$ this data to scalar-valued data:

$$
\mathbf{E} \stackrel{g}{\rightarrow} \hat{E}
$$

The scalar-valued measure $\hat{E}$ allows to visualize two- or three-dimensional patterns of deformation following, for instance, the color-scheme shown in figure 5.1, see also section 2.2 in chapter 2. Figure 5.2 shows the corresponding deformation measures that were used to depict dilating or contracting 
pieces of tissue throughout this thesis. The primary eigenvalue $\lambda_{1}(\mathbf{E})$ as well as the squared length of the material volume element or prism $\operatorname{tr}(\mathbf{E})$ was used as an estimate for the deformation state, in particular, to detect whether a material volume element was dilated with $\lambda_{1}(\mathbf{E})>0$ or $\operatorname{tr}(\mathbf{E})>0$ or compressed with $\lambda_{1}(\mathbf{E})<0$ or $\operatorname{tr}(\mathbf{E})<0$ :

$$
\hat{E}= \begin{cases}\text { dilated } & \text { for } \operatorname{tr}(\mathbf{E})>0, \lambda_{1}(\mathbf{E})>0 \\ \text { contracted } & \text { for } \operatorname{tr}(\mathbf{E})<0, \lambda_{1}(\mathbf{E})<0\end{cases}
$$

Accordingly, the derivative of the scalar-valued deformation measure $\partial_{t} \hat{E}$ was used to estimate the rate of deformation of the material volume element:

$$
\partial_{t} \hat{E}= \begin{cases}\text { dilating } & \text { for } \operatorname{tr}(\mathbf{E})>0, \lambda_{1}(\mathbf{E})>0 \\ \text { contracting } & \text { for } \operatorname{tr}(\mathbf{E})<0, \lambda_{1}(\mathbf{E})<0\end{cases}
$$

Note that here the volume was considered to be preserved with $\operatorname{det}(\mathbf{F})=0$. This chapter presents three-dimensional time-varying or four-dimensional simulation data. The data is scalar-valued due to the above described data reduction. For convenience, the use of capital letters $(X, Y, Z)$ for description of the material coordinates is omitted. see also section 2.2 in chapter 2. Throughout this chapter, the coordinate $(x, y, z)$ refers to the material coordinate $(X, Y, Z)$ of the continuum body if not stated otherwise.

\subsection{Planar Electromechanical Wave Propagation}

Planar electromechanical wave propagation was simulated in cubic-shaped bulk media by setting the excitatory variable $u$ of all units on one side of the cube above threshold. As a result, a planar electrical wave would propagate across the medium, see figures 5.3 and 5.4. In the following figures, the shape of the deforming medium is indicated by gray edges. The electrical wave is indicated in green using a ray-casting volume rendering technique that renders opaque green color at locations with $u>0.1$ and transparent empty space otherwise. The muscle fiber alignment is indicated by grey arrows. The internal Green-Lagrangian strain $\mathbf{E}$ is indicated using the red-white-blue colorcode presented in figure 5.1 and the data reduction $\hat{E}=g(\mathbf{E})$ as discussed in the previous section. Similarly as for the excitatory variable $u$, ray-casting volume rendering is used to indicate dilating material segments in blue and contracting material segments in red and undeformed material segments as transparent empty space respectively. The visualization technique allows to display both time-varying, three-dimensional scalar fields of the electrical activity $u(x, y, z, t)$ and the mechanical activity $\hat{E}(x, y, z, t)$.

Figure 5.3 shows planar electromechanical wave propagation in a cubic-shaped bulk medium with uniform linearly transverse muscle fiber alignment along the horizontal $\mathbf{e}_{x}$-axis (gray arrows). The electrical wave (green) starts spreading into the cube from the left face of the cube and traverses the cube along the $\mathbf{e}_{x}$-axis as a planar wave with the wavelength being comparably large to the dimensions of the cube. Note that the wave propagation direction coincides with the direction of the fiber alignment. As the wave traverses the cube it deforms accordingly. The tissue within and behind the electrical wave front experiences shortening due to cardiac cells contracting. As a result, 


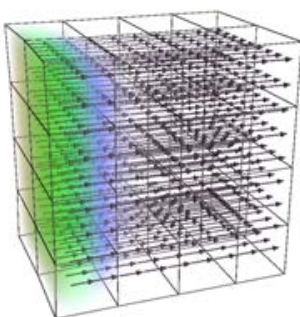

200

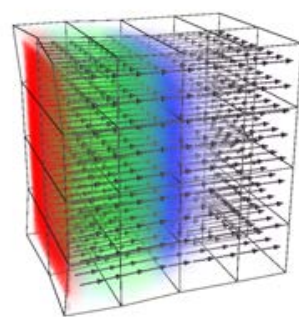

480

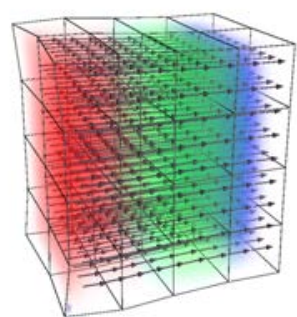

840

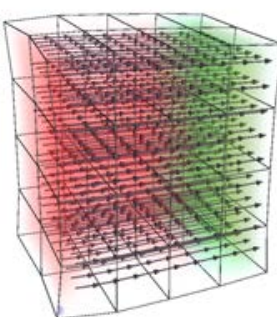

1120

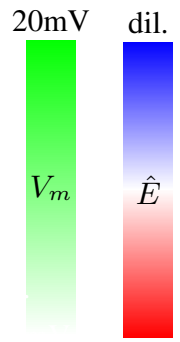

$-80 \mathrm{mV}$ contr.

Figure 5.3: Planar electromechanical wave propagation in bulk medium with uniform, linearly transverse muscle fiber alignment and wave propagation direction coinciding with fiber alignment: dynamic behavior of the contraction exhibits strong gradients between contractile and dilated regions inside the tissue matching the orientation and propagation direction of electrical wave (electrical wave: green normalized units of excitation $[0,1] \rightarrow[-80 \mathrm{mV}, 20 \mathrm{mV}]$, dilation: blue, contraction: red).

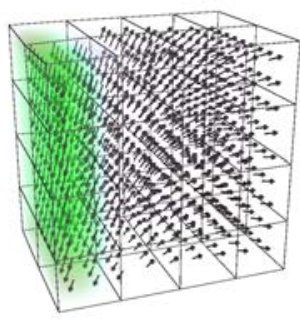

200

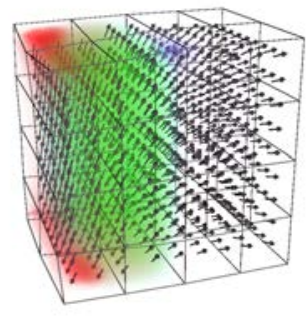

480

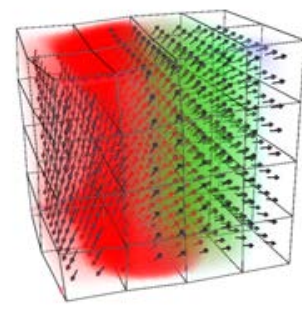

840

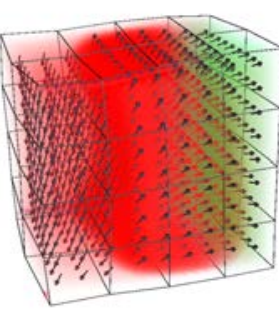

1120

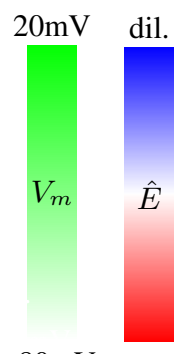

$-80 \mathrm{mV}$ contr.

Figure 5.4: Planar electromechanical wave propagation in bulk medium with rotating muscle fiber alignment; most fibers perpendicular to the propagation direction of the wave (electrical wave: green normalized units of excitation $[0,1] \rightarrow[-80 \mathrm{mV}, 20 \mathrm{mV}]$, dilation: blue, contraction: red).

one can observe a compressile strain wave front inside and behind the electrical wave front, indicated in red. Tissue in front of the approaching electrical wave front, experiencing long range elastic effects, is being pulled towards the wave front and dilates. One can observe a tensile strain wave front, indicated in blue, in front of the electrical wave. Overall, one can observe two clearly distinct tissue regions, one within and one in front of the wave, experiencing compressile and tensile strain respectively, with a strong gradient between the two regions. This effect is particularly strong as the contraction is directed uniformly along the $\mathbf{e}_{x}$-axis. Note that the deformation pattern corresponds to the picture postulated in figure 5.1. Figure 5.4 shows planar electromechanical wave propagation in a cubic-shaped bulk medium with rotational anisotropic muscle fiber alignment. Again, the electrical wave (green) starts spreading into the cube from the left face of the cube and traverses the cube along the $\mathbf{e}_{x}$-axis as a planar wave with the wavelength being comparably large to the dimensions of the cube. However, this time the wave propagation direction does not coincides with the fiber alignment. The fiber configuration is not uniform, but rotates and is aligned at most locations perpendicularly to the propagation direction of the electrical wave. Accordingly, the deformation is very different from the one shown in figure 5.3, and, in particular, the strong gradient between dilating and contracting regions of the tissue can not be observed. Nevertheless, one can observe a compressile strain wave front inside and behind the electrical wave front, indicated in red.

Planar electromechanical wave propagation can be observed in the heart during pacing, using stim- 

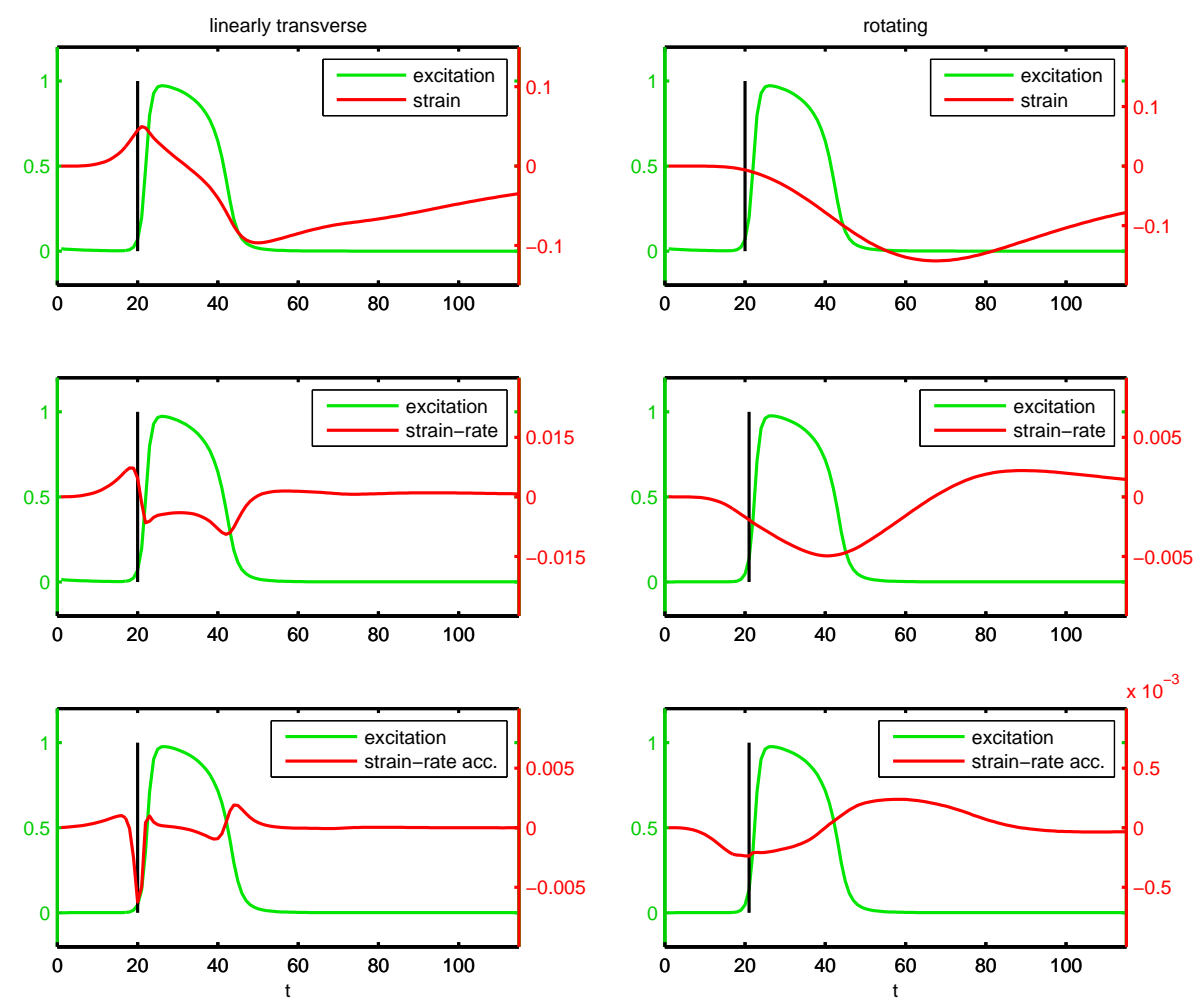

Figure 5.5: Elastic behavior of central material volume element during trespassing of planar electrical wave (green) in elastic excitable cubic-shaped bulk medium with uniform linearly transverse (left, see also figure 5.3) and rotating muscle fiber alignment (right, see also figure 5.4): the dynamic behavior of the elasticity (red) allows to estimate the trespassing of the wave (black bar).

ulation electrodes and applying stimulation pulses at one site on the ventricular surface to induce electrical wave activity that spreads over the ventricles away from that site. During sinus activity, the electrical activity starts spreading from multiple sites, which correspond to the ejection sites of the Purkinje system. The simulations were conducted with cubic-shaped bulk media of size $41 \times 31 \times 41$ particles or $40 \times 30 \times 40$ hexaedral cells using equations 2.1.13-2.1.14. Parameters were set to $a=0.05, b=0.5, \mu_{1}=0.1, \mu_{2}=0.3, k_{T}=1.9, k=8.0, \varepsilon=0.01, D=0.001, d t=0.02$. Simulations were run for 3000 time steps. Note, that the wavelength is comparably large as the dimensions of the cube.

\subsubsection{Muscle Fiber Anisotropy}

The two figures 5.3 and 5.4 demonstrate that deformations can differ significantly from each other if the tissues retain different underlying muscle fiber configurations. In particular, the deformations can differ from each other even though the underlying electrical pattern causing the deformation is the exact same pattern, compare 5.3 and 5.4. The muscle fiber anisotropy of the cardiac substrate determines the deformation and its time-varying behavior during the contraction, compare also with the work by Otani et al. ${ }^{192}$ This behavior strongly determines how deformation patterns emerge inside the cardiac muscle and affects how the pattern can be analyzed to reconstruct the underlying 
electrical wave pattern, see following section. The simulations show that the angle between local muscle fiber orientation and the wave normal of the electrical wave determines the strength of the deformation around that area inside the tissue.

\subsubsection{Wave Front Detection from Dynamic Features of Elasticity}

Figure 5.5 shows the according traces of different deformation measures obtained from the central material volume element of each of the two bulk media that retain differing muscle fiber configurations. In addition to the deformation state, that is the strain derived from the primary eigenvalue or the squared length of the deformation tensor $\mathbf{E}$, see also section 5.1.1, the rate of deformation or strain-rate as well as the strain-rate acceleration are shown (from top to bottom). All three traces exhibit strongly dynamic behavior with the trespassing of the electrical wave, for the case of uniform linearly transverse fiber alignment as well as for the case with rotational muscle fiber alignment. The strain undergoes a transition from dilated to compressed deformation states. The strain-rate accordingly switches sign from dilating to contracting rates of deformation at the peak of the dilation with high rates of change and a negative peak in the strain-rate acceleration accordingly. This dynamic elastic behavior allows the automatic detection of the trespassing of the electrical wave as the average time of the peak of dilation and the zero-crossing of the strain-rate from dilating to contracting rates of deformation and the fastest contracting acceleration rates of deformation coincides very well with the upstroke of the electrical wave front, see black bars in figure 5.5. In the following sections, this techniques is used to detect electromechanical wave fronts during arbitrary electromechanical wave activity. For every transition of an electrical wave through each material point inside the tissue, it is possible to observe a sequence of stretching, shortening and relaxation of the tissue with the rate of deformation becoming extremal with the onset of contraction. Accordingly, the above described detection technique reveals the isochronal spatial-temporal structure of periodic activation pattern.

Note that the order of magnitude of the rate of deformation is smaller in the case of rotational muscle fiber alignment. As the wave propagation direction and the local muscle fiber alignment differ, the aforementioned effects are not as pronounced and the detection of the trespassing of an electromechanical wave may become superimposed by other elastic activity if analyzing noisy data obtained in experiments with real cardiac tissue preparations.

\subsection{Electromechanical Spiral and Scroll Wave Patterns}

Spiral and scroll wave patterns are conjectured to underlie cardiac arrhythmias, see sections 1.1 and 1.3.3. This section studies coupled electromechanical patterns that arise in two- and threedimensional elastic excitable media with underlying electrical spiral and scroll wave activity respectively. It is demonstrated that separate analysis of electrical and elasto-mechanical activity of coupled electromechanical spiral and scroll wave activity reveals a high degree of similarity of the two patterns. The analysis allows the localization of spiral and scroll wave cores from the mechanical activity in deforming cardiac tissue.

\subsubsection{Two-dimensional Spiral Wave Patterns}

Spiral wave patterns occur in two-dimensional excitable media, see also section 1.1 and 2.1. They can be observed experimentally in the petri-dish when analyzing video data of, for instance, rotating 


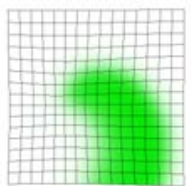

0

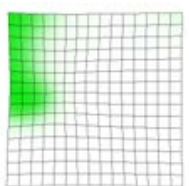

60

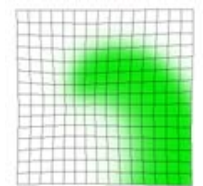

10

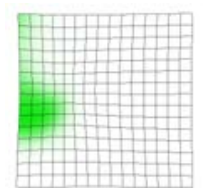

70

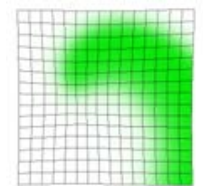

20

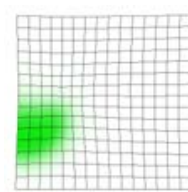

80

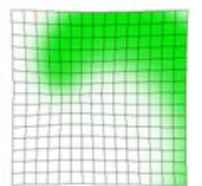

30

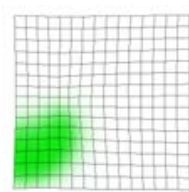

90

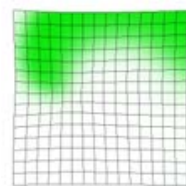

40

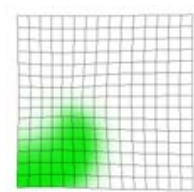

100

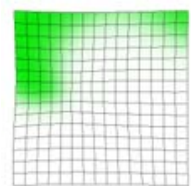

50

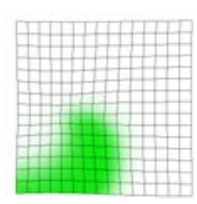

110
$20 \mathrm{mV}$

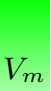

$-80 \mathrm{mV}$

$20 \mathrm{mV}$

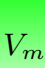

$-80 \mathrm{mV}$

Figure 5.6: Electrical spiral wave (green) exhibiting counter-clockwise rotations in two-dimensional elastic excitable medium with horizontal linearly transverse muscle fiber anisotropy: spiral core remains in approximate same position.

electrical activity such as action potential or calcium transient waves in two-dimensional monolayer cardiac cell culture preparations, ${ }^{136,146,154}$ see also section 1.1. Spiral waves organize dynamically around a center of rotation, which is a singular point towards the core region or tip of the spiral. This point presents a topological defect of the spiral wave pattern and can generally be stationary or move in space depending on the dynamical behavior of the spiral wave. ${ }^{56,58}$ Spiral wave cores are often used to depict the topological structure of the spiral wave pattern, as they are understood to be the organizing centers of spiral waves. As spiral wave activity can be composed of many spiral wave rotors, they can therefore serve to effectively simplify the complexity ${ }^{125}$ of the pattern and to locate the organizing centers. Here, it is shown that, in analogy to the spiral wave cores observed in excitable media, it is possible to identify similarly singular points inside the elasto-mechanical patterns that arise in elastic excitable media with underlying electrical spiral wave activity around the core region of the spiral. This finding allows to describe the complex spatial-temporal elastic behavior of deforming elastic excitable media with underlying spiral wave activity similarly as described prevoiusly ${ }^{125}$ for electrical spiral wave activity in excitable media. Moreover, it allows to identify the topology of the electrical pattern in case that the electrical pattern can not be observed directly.

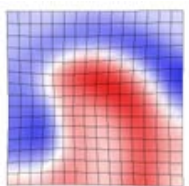

0

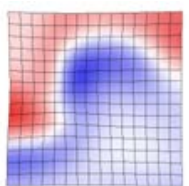

60

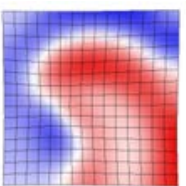

10

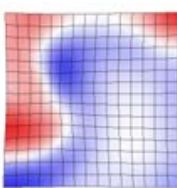

70

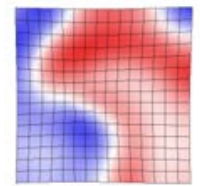

20

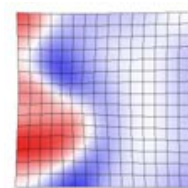

80

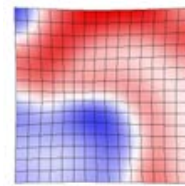

30

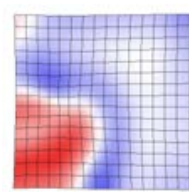

90

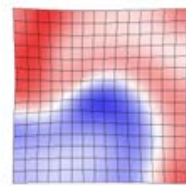

40

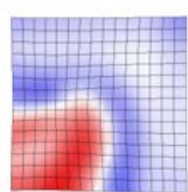

100

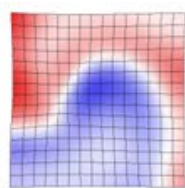

50

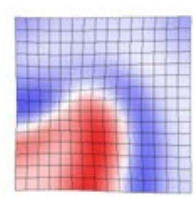

110

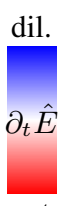

contr.

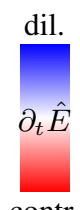

contr.

Figure 5.7: Counter-clockwise rotating elasto-mechanical deformation pattern induced by the spiral wave activity shown in figure 5.6 above. Blue-White-Red colorcode indicates dilating and contracting rates of deformation. Largest rates of deformation are localized within the electrical wave where medium contracts. Spiral wave-like shape resembles shape of electrical spiral wave. 


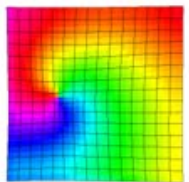

0

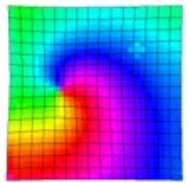

60

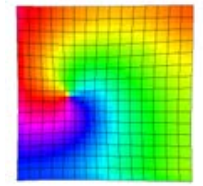

10

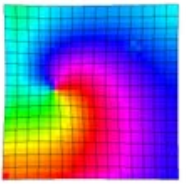

70

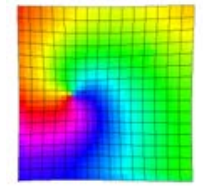

20

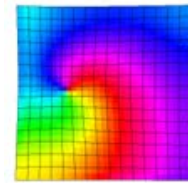

80

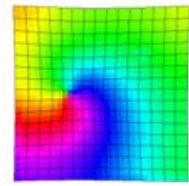

30

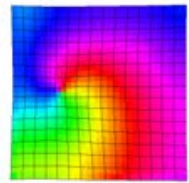

90

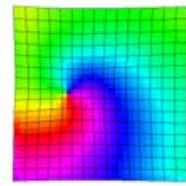

40

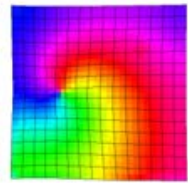

100

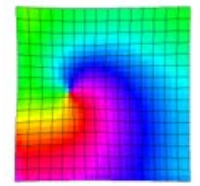

50

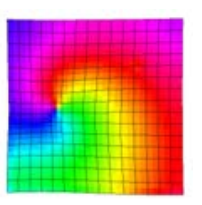

110
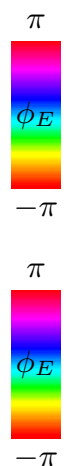

$-\pi$

Figure 5.8: Phase representation of elasto-mechanical deformation pattern shown in figure 5.7 revealing a phase singularity at the center of the medium, which indicates the core region of the electrical spiral wave as well as the center of rotation of the elasto-mechanical deformation pattern. Continuous colorcode indicating phase state angle $\phi_{E}$ of mechanical activity ranging from $\phi \in[-\pi, \pi]$.

Figures 5.6-5.8 show spiraling electromechanical wave activity in a two-dimensional elastic excitable medium. The medium retains horizontally aligned, linearly transverse muscle fiber anisotropy and undergoes contractions and deformations according to the underlying counter-clock-wise rotating electrical spiral wave pattern (green), see figure 5.6. The electrical spiral wave core remains stationary located approximately at the center of the medium and the wavelength of the electrical wave is comparably large to the size of the medium. The time-varying elasto-mechanical deformation pattern, see figure 5.7, exhibits similarly counter-clock-wise rotating spiral wave-like shaped patterns of deformation with local regions of the medium experiencing periodically dilating or contracting rates of deformation. The transition from dilating to contracting rates of deformation forms an isochronal line that takes on the shape of the electrical spiral wave front and is co-localized with the actual electrical spiral wave front. Gradients between regions of dilating and contracting rates of deformation become largest along the horizontally aligned muscle fiber direction. The largest compressile rates of deformation (red) occur within the electrical wave as the electrical wave is followed by an onset of active contractile activity.

The simulations were conducted with square-shaped two-dimensional media of size $41 \times 41$ particles or $40 \times 40$ rectangular cells respectively using equations $2.1 .13-2.1 .14$ to simulate waves of electrical excitation. Parameters were set to $a=0.09, b=0.5, \mu_{1}=0.1, \mu_{2}=0.3, k_{T}=1.5, k=8.0$, $\varepsilon=0.01, D=0.001, d t=0.002$. Simulations were run for 3,000 time steps and every 20th timestep was stored. The images above show the activity starting from time step 500. Note, that the wavelength is comparably large as the dimensions of the medium.

\section{Phase Representation and Phase Singularity}

As the elastic medium experiences a sequence of stretching, shortening and relaxation and as this sequence repeats periodically during sustained spiral wave activity, it becomes possbile to translate the time-varying deformation pattern into a pattern that continuously maps the local deformation state to an angular, continuous phase state $\phi \in[-\pi, \pi]$. This so-called phase representation or phase portrait of the pattern can be derived due to the periodicity of the activity. Phase representations are typically used to reduce the complexity ${ }^{59}$ and to reveal the rotational nature of spiral wave patterns in excitable 
media and they similarly allow to compute phase singular points within the phase-mapped patterns to determine the locations of spiral wave cores. ${ }^{59,125}$ For instance, the excitatory dynamic variable $u$ oscillates periodically between $[0,1]$ during spiral wave activity and it is possible to define the phase of oscillation $\phi$, with an oscillation period $T$, that is given by the time between two subsequent activations of the unit or the times of two subsequent trespassings of the spiral wave through the point considered. The period $T$ corresponds to the period of one oscillation or excitatory excursion of the variable $u$ through the activation-recovery cycle and $f$ similarly corresponds to the rotational frequency of the spiral, see also section 2.1.4 in chapter 2. Typically, the time points of activation are associated with the upstroke of the excitatory variable. In between two upstrokes, the progress of the dynamic state variable $u$ in the activation-recovery cycle ${ }^{125}$ can be expressed as a phase state angle $\phi_{u}$, continuously mapped onto an interval of $\phi \in[-\pi, \pi]$. In two spatial dimensions, a wave front then corresponds to an isochronal line of activation with $\phi=-\pi$ in the phase representation of the activity. All other isochronal lines of activation are lines of equal phase, with the phase state angle being derived from the continuously interpolated phase map $\phi(x, y)$. Spiral wave activity corresponds to spiral wave-shaped isochronal lines of activation. Phase singular points correspond to sites at which all phase values $\phi \in[-\pi, \pi]$ or lines of equal phase converge. ${ }^{59}$ These phase singular points can be computed integrating the spatial gradient of the phase angles along a closed path around a site in the plane, see also section 2.1.4 in chapter 2. The integral yields non-vanishing sums around phase singular points ${ }^{59}$ and assuming that the enclosed path does not enclose two phase singular points with opposite topological charge, which would also lead to a vanishing integral.

Here, the above described concept was adapted to derive phase representations of elasto-mechanical deformation patterns, like the one shown in figure 5.7. The phase representation displays the rotational topology of the deformation pattern and allows to determine phase singular points or topological defects within the pattern. Figure 5.8 shows the phase portrait of the elasto-mechanical deformation pattern shown in figure 5.7. The phase mapping was achieved by introducing a phase shift from $-\pi$ to $\pi$ for every moment in time in which the rate of deformation changes from dilating rates of deformation to contracting rates of deformation, to obtain an isochronal line that marks the onset of contraction. In between, the phase angle was interpolated linearly. Figure 5.8 displays the phase shifts from $-\pi$ to $\pi$ in red and all other phase angles $\phi$ in a continuous HSV-colorcode. The pattern exhibits a vortex structure with lines of equal phase spiraling from the center away to the outside of the medium. At the center of the medium, all lines of equal phase converge and indicate a phase singularity inside the pattern. The pattern rotates counter-clock wise around the phase singularity and the phase singularity remains stationary. The phase singularity marks a topological defect inside the time-varying deformation pattern, which is co-localized with the electrical spiral wave core.

The analysis shows that coupled electromechanical spiral wave activity may exhibit phase singularities in both the electrical as well as the mechanical phase portraits of the electromechanical pattern. These phase singular points may be co-localized and may similarly indicate the core region of the electromechancial spiral wave pattern and organizing center of the activity. These findings suggest that it is feasible to understand electromechanical spiral waves to be composed of electrical as well as mechanical spiral waves. Moreover, analysis of the mechanical activity allows to reveal the organizing structure of the electrical activity. 


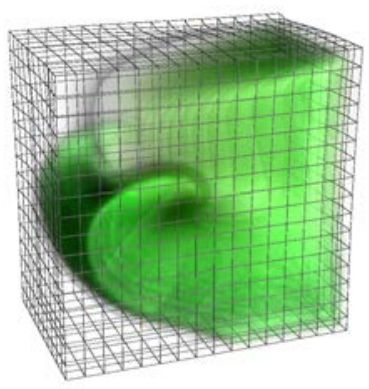

80

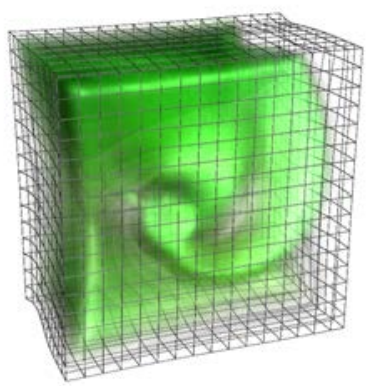

220

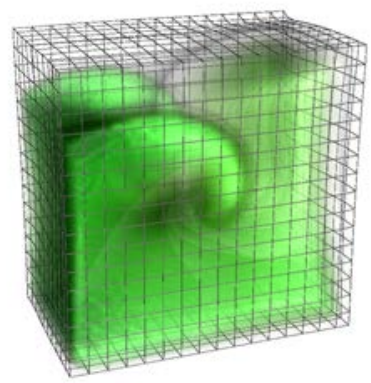

130

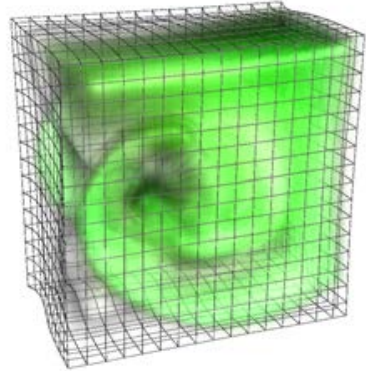

290
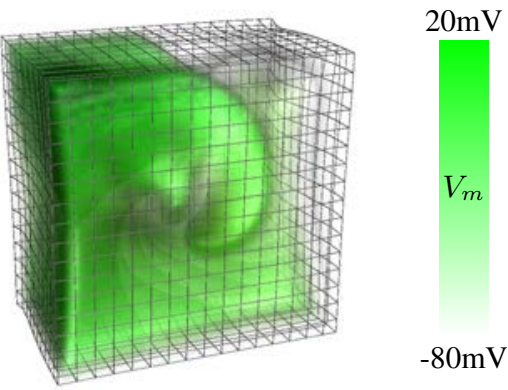

170
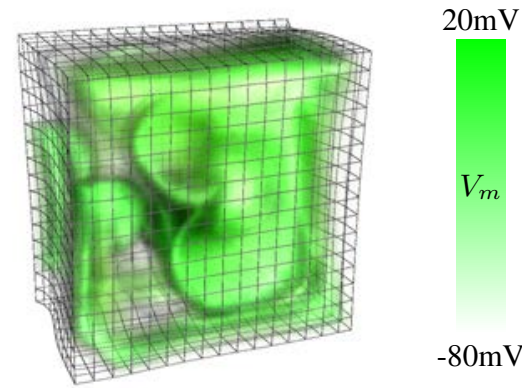

510

Figure 5.9: Electrical scroll wave activity in accordingly deforming elastic excitable cubic-shaped bulk medium with rotational underlying muscle fiber anisotropy: clock-wise scroll wave (green) reentry and break-up into multiple scroll waves. Volume rendering with excited segments displayed in green and refractory or non-excited segments displayed in transparent.

\subsubsection{Three-dimensional Scroll Wave Patterns}

Scroll waves are the three-dimensional equivalent to spiral waves, see also sections 1.1 and 2.1.4. They organize dynamically around lines of phase singularity, so called vortex filaments, which can be arbitrarily curved lines in three-dimensional space. Figure 5.9 shows electrical scroll wave activity (green) in a deforming elastic excitable, cubic-shaped bulk medium with rotational, underlying muscle fiber anisotropy. Scroll wave activity was initiated with one planar electrical wave traveling from the left to the right side of the cube and erasing all excitatory activity in the lower half of the cube in the moment as the wave traveled half of the distance to the other side of the cube, similarly as described in section 4.1. As a result, one scroll wave rotor performs clock-wise reentry within the medium around an axis parallel to the short $\mathbf{e}_{z}$-axis of the bulk, see time step 80 in figure 5.9 or 5.11. The wavelength of the electrical scroll wave is comparably large to the size of the medium. Here, two situations were studied: (1) the rotor breaks up into multiple other scroll wave rotors quickly, before the completion of one full rotation, see time step 170 in figure 5.9. The activity develops into progressively disordered, turbulent electrical activity composed of multiple rotors. The situation was thought to resemble the emergence of spatial-temporal chaos during ventricular fibrillation. (2) The rotor is stable and does not break up, but performs many rotations with its rotational core region staying approximately at the center of the medium. This situation was thought to resemble rotor activity during ventricular tachycardia. 


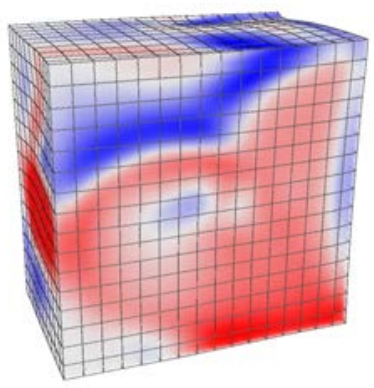

20

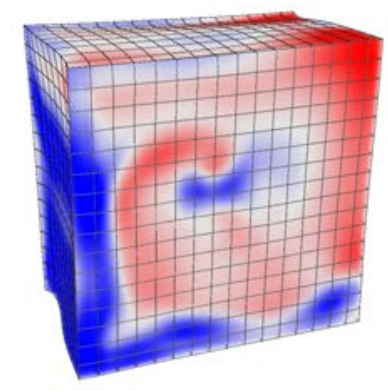

220

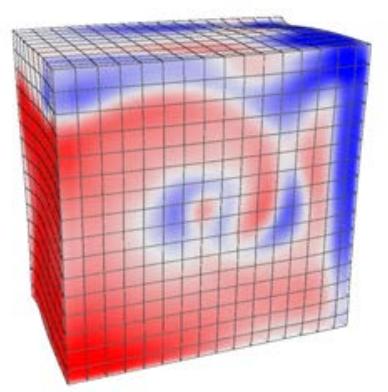

80

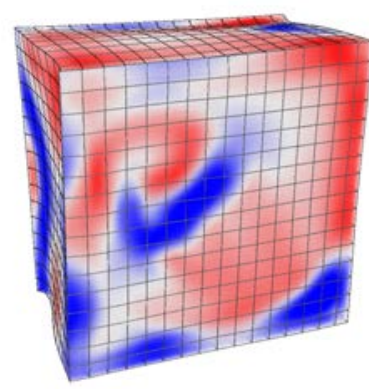

430
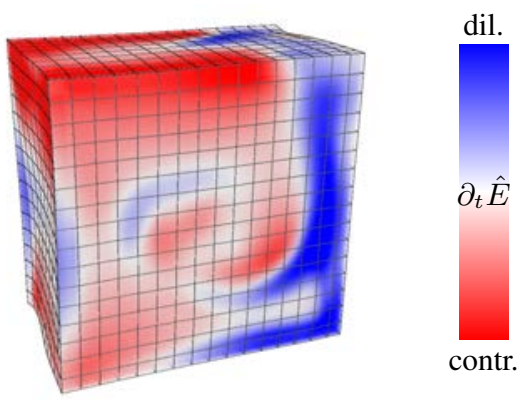

140
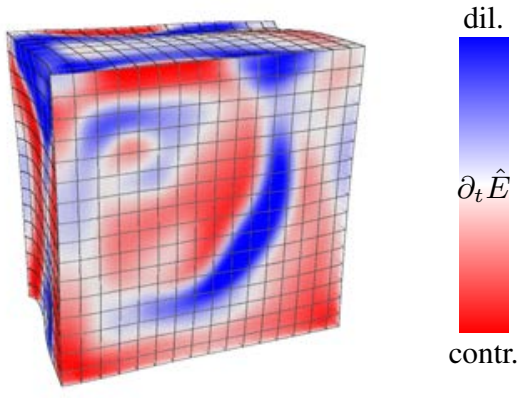

520

Figure 5.10: Deforming elastic excitable bulk medium with underlying electromechanical scroll wave activity as shown in figure 5.9 and rotational muscle fiber anisotropy: tissue exhibits regions with dilating (blue: $\hat{E}>0$ ) and contracting (red: $\hat{E}<0$ ) rates of deformation that resemble rotating scroll wave-like patterns.

The electrical scroll wave activity triggers contractile activity, which causes the bulk to deform accordingly, see figure 5.10. The underlying three-dimensional configuration of muscle fibers consists of sheets of equal muscle fiber alignment stacked orthotropically along the short $\mathbf{e}_{z}$-axis of the cube, see figure 5.12(c). Hence, all fibers are aligned within the $x$-y-plane, uniformly parallel in one sheet. However, the average muscle fiber alignment of each sheet rotates through the stack of sheets, in total by an angle of $120^{\circ}$. The muscle fiber configuration is supposed to mimick the rotational muscle fiber anisotropy of the ventricular wall, see figures 5.12(a) and 4.4 in section 4.4 in chapter 4 .

Figure 5.10 shows the internal, volumetric time-varying elasto-mechanical deformation pattern emerging inside the bulk as the electrical activity induces contractile deformations and the scroll wave rotor breaks up. The pattern was derived by saving the time-varying three-dimensional scalar-valued deformation field data $\hat{E}(x, y, z, t)$ for every material coordinate from the simulation output data. This step required data reduction of tensor-valued data to scalar-valued data, as described in section 5.1.1. The time-varying three-dimensional rate of deformation field $\partial_{t} \hat{E}(x, y, z, t)$ was computed accordingly and smoothed using four-dimensional spatial-temporal filtering (kernel size $k_{x}=k_{y}=7$ pixels diameter, $k_{t}= \pm 12$ frames). The elasto-mechanical deformation pattern exhibits similarly as depicted in figure 5.7 a rotational, spiral wave-like structure on the surface. However, further interpretation of the pattern remains difficult.

Figure 5.11 shows a direct comparison of the electrical and elasto-mechanical deformation patterns for a less complicated dynamical pattern - a single, non-meandering, stable scroll wave rotor with 


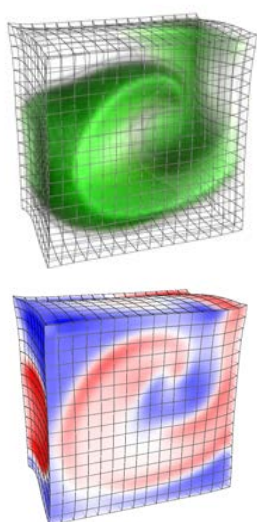

80
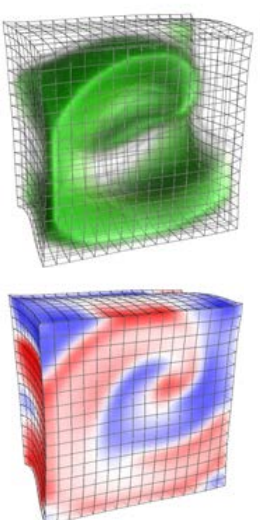

90
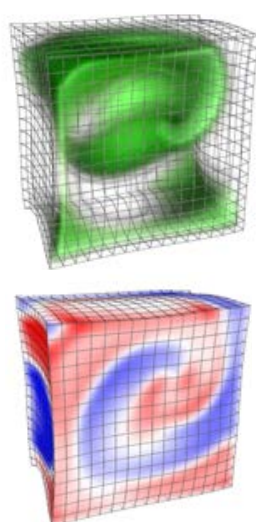

100
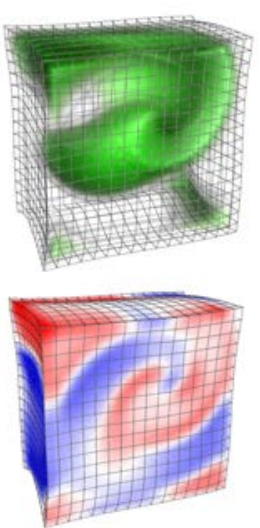

110

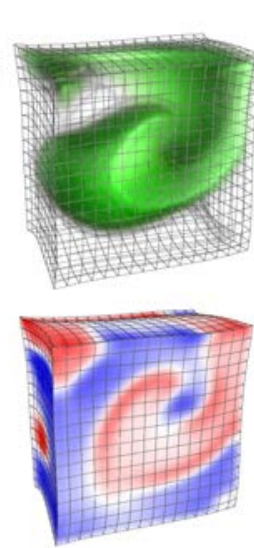

120
$20 \mathrm{mV}$
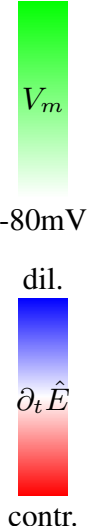

Figure 5.11: Single, stable and stationary scroll wave with curved rotational core region: the electrical scroll wave induces deformations of the bulk, that - if depicted as rate of deformation patterns (strainrate) - are highly similar to the electrical scroll wave pattern. The simulation suggests that the internal, volumetric, time-varying deformation field within the bulk of the medium retains a similar topological structure as the electrical pattern with a rotational core region that is similar to the electrical one.

curved rotational core. For this situation, the elasto-mechanical deformation pattern clearly retains a spiral wave-like appearance. The mechanical deformation pattern suggests that the internal, volumetric, time-varying deformation field within the bulk of the medium retains a similar topological structure as the electrical pattern, with a rotational core region that is similar to the electrical one.

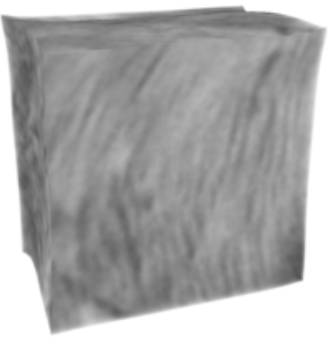

(a)

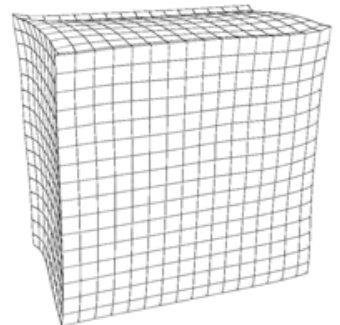

(b)

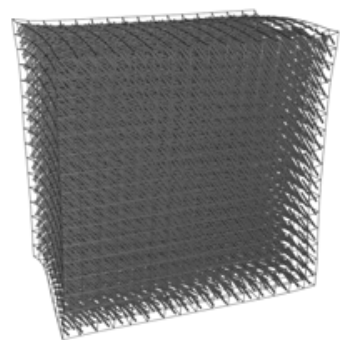

(c)

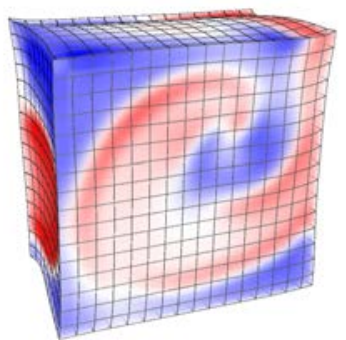

(d)

Figure 5.12: Muscle fiber anisotropy: (a) $\mu$-CT-scan of right ventricular wall of rabbit heart (about $1.5 \times 1 \times$ $1.5 \mathrm{~mm}$ ) revealing muscle fiber anisotropy, the volumetric imaging data is deformed according to the simulated deforming bulk medium (b) deformed simulation grid (c) underlying muscle fiber configuration of simulation shown in figures 5.9, 5.10 and 5.11 (d) volumetric anisotropic strain-rate pattern, anisotropy of the pattern correlated with underlying muscle fiber organization

Figures 5.10 and 5.11 show that the emergence of the elasto-mechanical pattern with its highly dynamic elastic features develops similar patterns as the electrical activity. The similarity of the two patterns suggests that the mechanical activity constitutes the mechanical fingerprint of the electrical scroll wave pattern. In particular, the patterns exhibit similarity considering even the highly anisotropic underlying muscle fiber configuration, see figure 5.12. 
Chapter 5. Electromechancial Wave Pattern Reconstruction

The simulations were conducted with cubic-shaped three-dimensional elastic excitable bulk media of size $81 \times 51 \times 81$ particles or $80 \times 50 \times 80$ voxels or hexaedral cells respectively using equations 2.1.17-2.1.22 to simulate the waves of electrical excitation shown in figure 5.9 and equations 2.1.13-2.1.14 to simulate the waves of electrical excitation shown in figure 5.11. The muscle fiber configuration was set to a similar configuration as shown in figure 5.12(c) with the sheet orientation rotating by $120^{\circ}$ through the wall. Parameters for the integration of the equations 2.1.17-2.1.22 were set to the values provided by table B.2 together with $k_{T}=1.8, k_{h}=64, \varepsilon=0.01, D=0.0006$, $d t=0.001$. Parameters for the integration of the equations 2.1.13-2.1.14 were set to $a=0.9$, $b=0.2, \mu_{1}=0.1, \mu_{2}=0.3, k=8.0, k_{T}=3.0, k_{h}=32, \varepsilon=0.01, D=0.001, d s=0.01$, $d t=0.02$. Simulations were run for 100,000 time steps and every 50th or 20th timestep was stored respectively. Note, that the wavelength is comparably large as the dimensions of the medium.

\section{Scroll Wave Patterns in Heart-Shaped Geometries}

Figure 5.13 and 5.14 show electromechanical scroll wave activity in a realistically heart-shaped elastic excitable medium. The realistic anatomical, heart-shaped geometry was obtained from segmented computerized tomography scans, see section 4.4 in 4 , of rabbit hearts, which were also previously used in the experiments presented in chapter 6 . The simulation domain is separated into one domain that includes the volume of the ventricular walls, one that includes the volume of the atrial walls and one domain that includes other tissue, which is not elecrically excitable or conducting but is part of the entire connected elastic domain. In both figures, the upper panel shows the volumetric internal electrical activity (green) pattern and the lower panel shows the internal volumetric time-varying elasto-mechanical rate of deformation pattern with dilating (blue) and contracting (red) rates of deformation, see also section 5.1.1. The electrical activity occurs only in the ventricles, whereas the elastic deformation affects the entire heart.

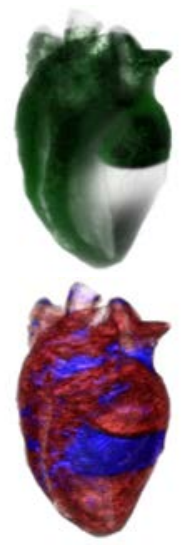

2

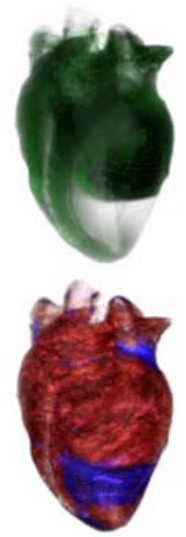

14
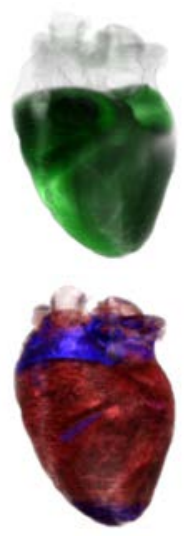

59
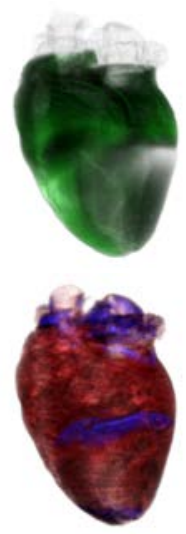

70
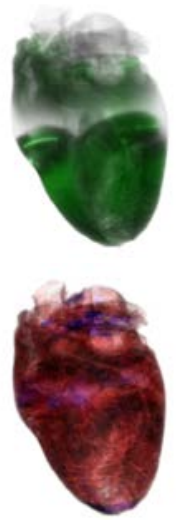

119

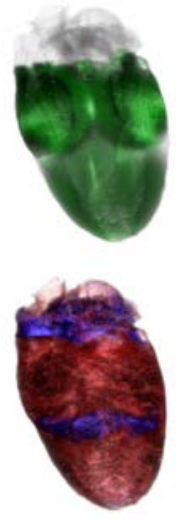

145

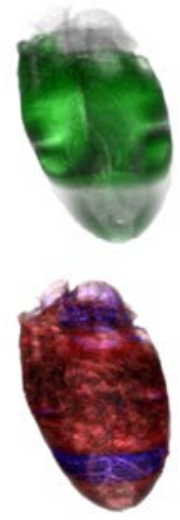

163

Figure 5.13: Electromechanical scroll wave activity in accordingly deforming realistically heart-shaped elastic excitable medium: scroll wave (green) reentry in left ventricular wall of rabbit heart, rate of deformation with dilating (blue) and contracting (red) rates of deformation

Figure 5.13 shows electromechanical scroll wave activity with large rotors with wavelengths similar to the size of the heart in the ventricles. The scroll wave activity is composed of a meandering double rotor. At the beginning of the sequence, one can observe a clock-wise rotating scroll wave that 


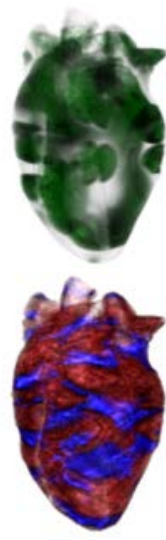

3
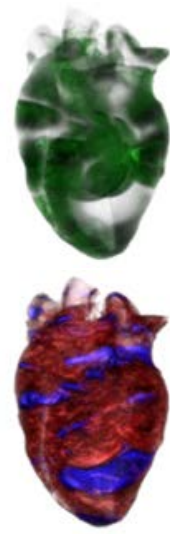

14
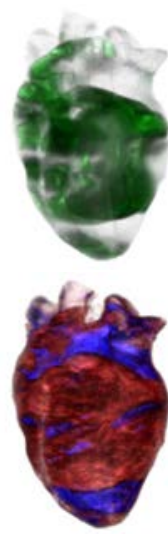

59
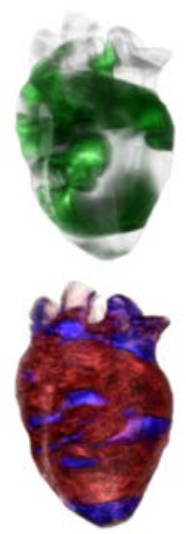

70
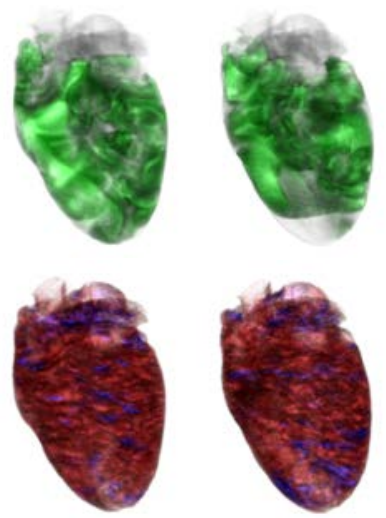

119

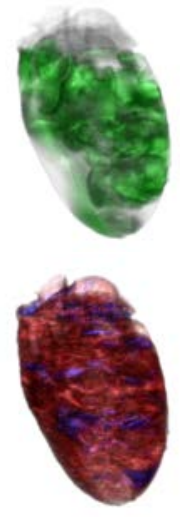

163

Figure 5.14: Highly chaotic electromechanical scroll wave activity in accordingly deforming realistically heart-shaped elastic excitable medium: scroll wave (green) activity in left ventricular wall of rabbit heart, rate of deformation with dilating (blue) and contracting (red) rates of deformation.

drifts inside the left ventricular wall towards the left side of the field of view, revealing that it belongs to one large double rotor, with the other rotor being a counter-clock-wise rotating scroll wave. The corresponding elasto-mechanical deformation pattern in the lower panel shows similarly large patterns of dilating and contracting rates of deformation, which seem to match the upper dynamics. For instance, in the first and second picture, one can see a large region experiencing dilating rates of deformation followed by contracting rates of deformation in front of the arm of the electrical scroll wave front, as it travels towards the apex of the heart. A similar effect can be observed in the last two pictures, as the double rotor is reentering the ventricle, leading to a deformation pattern similar to a figure-of-eight pattern.

Figure 5.14 shows electromechanical scroll wave activity with many small-scaled electrical scroll wave rotors. Over the sequence of the images, the number of rotors and the degree of disorder visibly increases, presumably as the dynamics in the beginning of the simulation are transient. Also the elasto-mechanical deformation pattern exhibits larger-scaled patterns in the beginning and then smaller scaled patterns in the end of the sequence accordingly.

Figures 5.13 and 5.14 show that the emergence of the elasto-mechanical pattern with its highly dynamic elastic features is robust and exhibits the characteristic pattern of dilating and contracting rates of deformation, even as the geometric shape of the medium becomes highly complex. Also, boundary effects of the elasticity do not seem to distort this pattern, even though the heart walls are relatively thin. A degree of similarity of the two electrical and mechanical patterns seems to be maintained at all times. Comparing figure 5.13 and 5.14 shows that the elasto-mechanical pattern depicts fundamental scales of the electrical spatial-temporal dynamics for large- as well as small-scaled dynamics. The large spatial scales of the less complicated electrical scroll wave pattern also causes large-scaled elasto-mechanical deformation patterns. The smaller scaled chaotic electrical pattern also causes small-scaled elasto-mechanical activity. This finding suggests that the analysis of cardiac mechanical activity may generally be used to characterize the underlying electrical activity. This has the potential to be used in inverse imaging applications, see section 5.5 and chapter 6 . 
Chapter 5. Electromechancial Wave Pattern Reconstruction

The simulations were conducted on a simulation grid of size $169 \times 171 \times 279$ voxels, with the tissue occupying about $25 \%$ of the voxels. The mechanical cells of the elastic particle system corresponded to tetrahedral cells, such that every voxel of the electrical grid was represented by 4 tetrahedras in the mechanical simulation. Parameters were set to the values provided by table B.2 for the scroll wave and chaotic case respectively. Other parameters were set to $d T=0.01 \mathrm{~ms}, k_{h}=64, \varepsilon=0.01$, $D=0.0006, d t=0.001$. For more information see also work by S. Stein. ${ }^{232}$ The fiber configuration was set to point along the vertical $\mathbf{e}_{y}$-axis. Note, that the wavelength is comparably large as the dimensions of the medium.

\subsubsection{Reconstructing Wave Front and Wave Pattern Topology}

In the previous sections, it was shown that the rapid onset of contraction during electromechanical wave activity can be associated with the electromechanical wave front and that the elastic behavior allows to derive isochronal lines in space that resemble the shape and topology of the activity, see figure 5.8. Here, this approach is studied more systematically. This section demonstrates how the detection of the isochronal structure of the three-dimensional elasto-mechanical deformation pattern within the volume of the actively contracting, deforming medium allows the reconstruction of the underlying three-dimensional electrical wave pattern during scroll wave activity. In three dimensions, isochrones correspond to isochronal surfaces. Isochronal surfaces can be reconstructed by detecting dynamic features of the elastic deformations in each material point of the medium, similarly as described in section 5.2.2. For the analysis, time-varying three-dimensional scalar-valued deformation field data $\hat{E}(x, y, z, t)$ was extracted for every material coordinate from the simulation output data. This step required data reduction of tensor-valued data to scalar-valued data, as described in section 5.1.1. Deformation rates $\partial_{t} \hat{E}(x, y, z, t)$ and accelerations $\partial_{t}^{2} \hat{E}(x, y, z, t)$ were computed accordingly, similarly as described in section 5.3.2.

Figure 5.15 shows both electrical and elasto-mechanical activity as time-series obtained from one site within the elastic excitable cubic-shaped bulk medium shown in figure 5.9. The medium deforms according to the underlying electrical scroll wave activity and rotational muscle fiber anisotropy. The time-series in the upper panel shows a train of action potential waves of the electrical activity (green). The time-series in the second and third panel show the strain-rate $\partial_{t} \hat{E}_{x y z}(t)$ and strain-rate acceleration $\partial_{t}^{2} \hat{E}_{x y z}(t)$ respectively, derived from deformations of the respective material volume element at position $(x=40, y=25, z=40)$. Both signals fluctuate around zero, with the frequency matching the frequency of the electrical activity. Dilating and contracting strain-rates and strain-rate accelerations larger and smaller zero are indicated in blue and red respectively. The strain-rate exhibits sharp positive peaks, whereas the strain-rate acceleration exhibits sharp negative peaks, which approximately coincide in time. Simple feature detection to determine the peaks within both time-series and association of two nearby peaks from both time-series with a zero-crossing in the strain-rate yields a triplet of discrete times $\left(t_{i}^{0}, t_{i}^{+}, t_{i}^{-}\right)$, that indicate together an elastic event, which can be associated with the trespassing of a wave. This procedure was repeated for every point with material coordinates $(x, y, z)$ and its respective time-series $\partial_{t} \hat{E}_{x y z}(t)$ and $\partial_{t}^{2} \hat{E}_{x y z}(t)$ inside the three-dimensional medium, such that for each point a number of elastic events with $i=1, \ldots N$ was registered with the time of each elastic event being the average time of each triplet:

$$
t_{i}^{x y z}=\frac{t_{i}^{0}+t_{i}^{+}+t_{i}^{-}}{3}
$$


Chapter 5. Electromechancial Wave Pattern Reconstruction
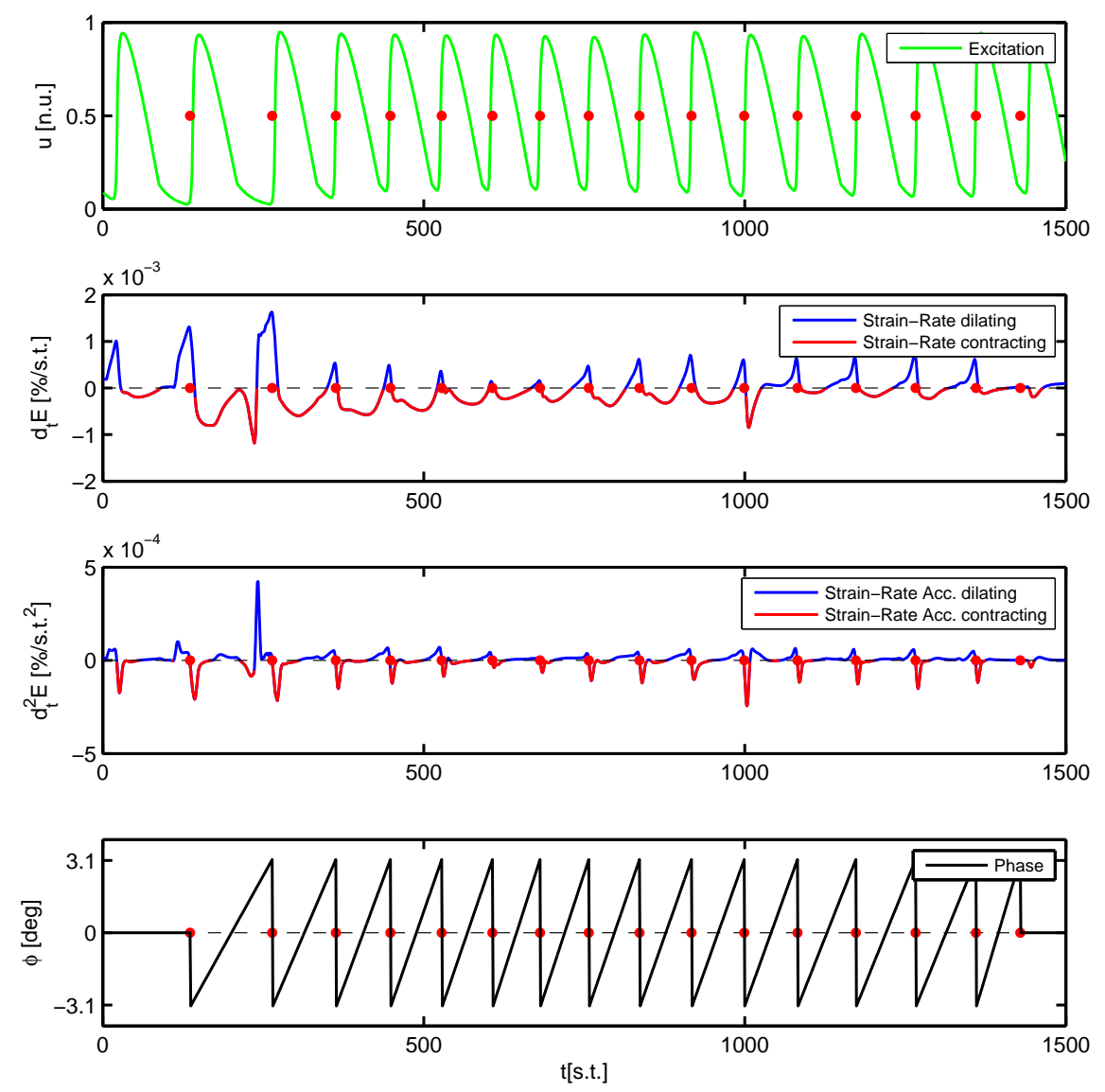

Figure 5.15: Time-series of electrical and elasto-mechanical activity during scroll wave activity within bulk medium shown in figures 5.9 and 5.10: train of action potentials (green, upper panel) and fluctuations of strain-rate (second panel) and strain-rate acceleration (third panel) with dilating (blue) and contracting (red) rates of deformation. Corresponding phase (lower panel) with phase shifts (red dots) introduced at discrete times $t_{i}$ that can be associated with extremal events in elasticity. Phase shifts located nearby upstrokes of action potentials. Data obtained from central site at coordinates $(x=40, y=25, z=40)$. 

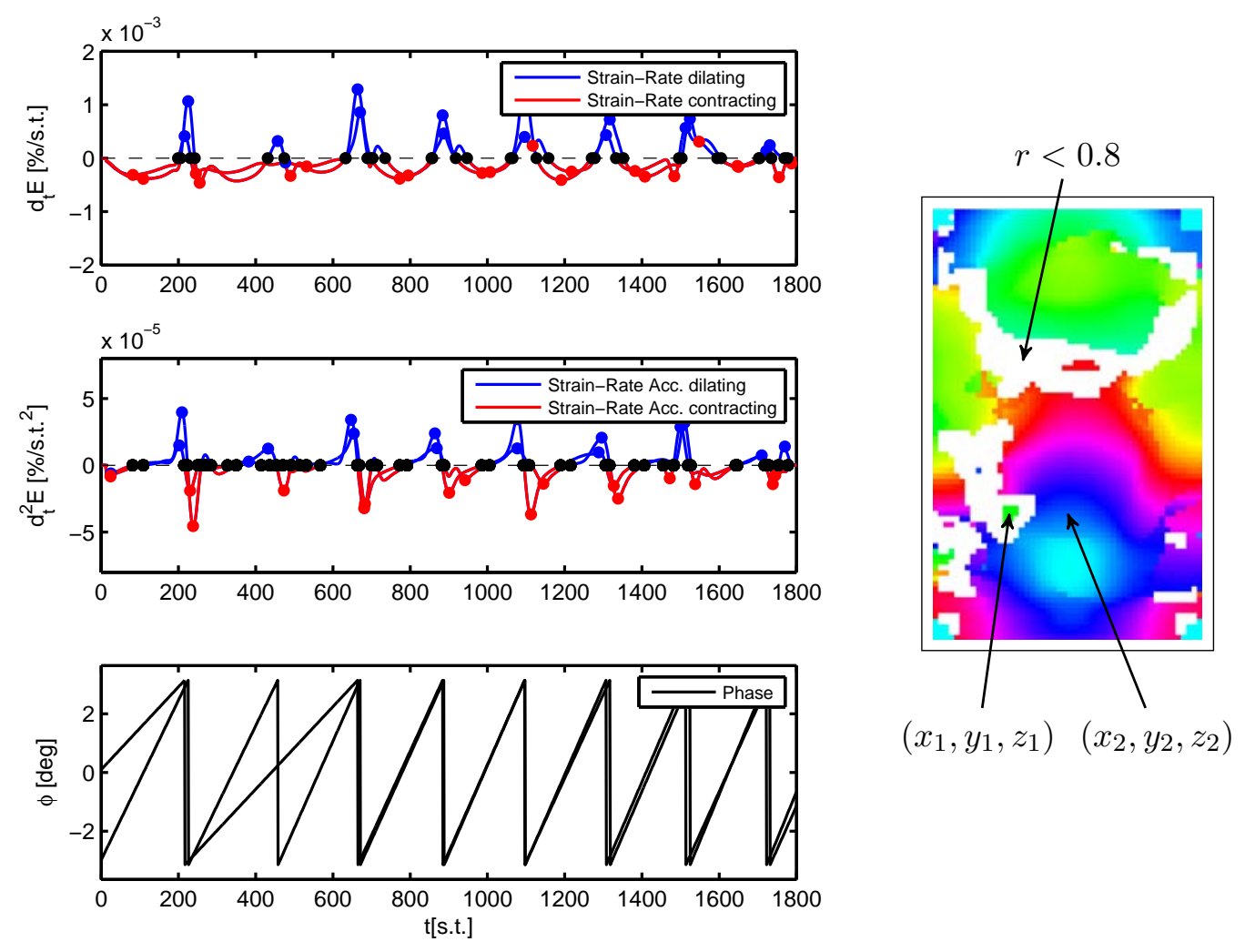

(a)

(b)

Figure 5.16: Reconstruction artifacts: discrete detection of extremal events in elasticity leads to phase mismatches and discontinuities. (a) time-series of strain-rate and strain-rate acceleration (dilating, contracting) from two nearby sites $\left(x_{1}=56, y_{1}=16, z_{1}=40\right)$ and $\left(x_{2}=56, y_{2}=25, z_{2}=\right.$ 40). (b) cross-section through three-dimensional reconstructed phase field $\phi_{E}(x=56, y, z, t=$ $358)$ with removed phase values with $r<0.8$ and locations of the sites indicated by arrows.

Consequently, the continuous time-varying, three-dimensional deformation fields $\partial_{t} \hat{E}(x, y, z, t)$ and $\partial_{t}^{2} \hat{E}(x, y, z, t)$ were reduced to a three-dimensional set of discrete times. These times were used to create a continuous three-dimensional time-varying phase representation $\phi_{E}(x, y, z, t)$ of the elastomechanical activity by introducing $2 \pi$ phase jumps from $-\pi$ to $\pi$ at all times $t_{i}$ as shown in the lower panel in figure 5.15. Interpolating linearly the phase values in between $t_{i}$ and $t_{i+1}$ produced a continuous phase representation field $\phi_{E}(x, y, z, t)$. As a result, the elasto-mechanical activity was translated into its three-dimensional time-varying phase representation:

$$
\hat{E}(x, y, z, t) \longrightarrow \phi_{E}(x, y, z, t)
$$

The phase jumps coincide with each of the upstrokes of the action potentials shown in the upper panel (indicated by red dots). The resulting phase representation $\phi_{E}(x, y, z, t)$ contains an isochronal structure, with a continuum of isochronal surfaces of equal phase. 


\section{Reconstruction Artifacts}

However, the above described analysis did not reliably capture all the upstrokes. The resulting threedimensional time-varying phase field $\phi_{E}(x, y, z, t)$ was found to be partially dicontinous, as a result of falsly detected or missed elastic events. Figure 5.16(a) shows the time-series of the elastic activity of two nearby sites $\left(x_{1}=56, y_{1}=16, z_{1}=40\right)$ and $\left(x_{2}=56, y_{2}=25, z_{2}=40\right)$ within the bulk medium, indicated in figure 5.16(b). The upper two panels show the strainrates $\partial_{t} \hat{E}_{1}(t)$ and $\partial_{t} \hat{E}_{2}(t)$ and strain-rate accelerations $\partial_{t}^{2} \hat{E}_{1}(t)$ and $\partial_{t}^{2} \hat{E}_{2}(t)$ of the two sites, plotted in the same graph on top of each other, whereas the lower panel shows the respective phase states $\phi_{E_{1}}(t)$ and $\phi_{E_{2}}(t)$ reconstructed from the above time-series. At $t=420$, the detection missed one elastic event in one of the time-series and did not introduce a $2 \pi$ phase jump. Filtering the phase representation field $\phi_{E}(x, y, z, t)$, using a four-dimensional phase coherence filter $\mathcal{F}^{\phi}$ produced a perforated phase state field $\tilde{\phi}_{E}(x, y, z, t)$, as it is depicted by the cross-section of the bulk shown in figure 5.16(b). The phase coherence filter involves converting every real-valued phase entry of the phase state field $\phi_{E}(x, y, z, t)$ into its complex decomposition:

$$
\phi_{E}(x, y, z, t) \longrightarrow \cos \left(\phi_{E}(x, y, z, t)\right)+i \cdot \sin \left(\phi_{E}(x, y, z, t)\right)
$$

and computing the order parameter $r(x, y, z, t)$ for the complex-valued three-dimensional neighbourhood $\mathcal{S}_{x y z}^{d}$ of every entry of the complex field:

$$
r \cdot e^{\phi}=\frac{1}{N} \sum_{j}^{N} e^{i \phi_{j}}
$$

where $\phi$ is the mean-field phase angle, $\phi_{j}$ are the $j=1, \ldots, N$ neighbouring complex-valued phase states inside the spherical-shaped neighbourhood $\mathcal{S}_{x y z}^{d}$ with diameter $d$ including the considered phase state at position $(x, y, z)$ itself and $0 \leq r \leq 1$ is the magnitude of the phase coherence of the ensemble of complex-valued phase states, which is commonly referred to as the order parameter $r$. The order parameter was determined for each phase field entry and the filter removed and replaced all phase field entries $\tilde{\phi}_{E}(x, y, z, t)$ by NaNs with a corresponding order parameter $r(x, y, z, t)$ below a threshold $r<0.9$ for a neighbourhood with diameter $d=3$ pixels or $r<0.8$ for a neighbourhood with diameter $d=5$ pixels. Interpolating all removed entries yielded a cleaned phase representation field $\phi_{E}$ of the elasto-mechanics, see following section. To further increase the quality of the phase representation field, other post-processing steps were added before applying the phase coherence filter. For instance, determining the average period $\bar{T}$ in between all subsequent times $t_{i=1, \ldots N}$ that were detected and used for phase-shifting:

$$
\bar{T}=\frac{1}{N-1} \sum_{i}^{N-1}\left|t_{i}-t_{i+1}\right|
$$

allowed to prefilter the data and increase the efficiency of artifact removal. The average period $\bar{T}$ was used to determine deviations in the periods in between two subsequent phase jumps $t_{i}$ and $t_{i+1}$ to either include missed entries or to remove entries that were falsly detected (deviations larger than $20 \%$ of the standard deviation).

Figures 5.17, 5.18 and 5.19 show the time-varying three-dimensional phase representations $\phi_{u}$ and $\phi_{E}$ of the electrical and mechanical activity inside cubic-shaped bulk as well as realistically heartshaped elastic excitable media. The phase representation of the electrical activity $\phi_{u}(x, y, z, t)$ was 

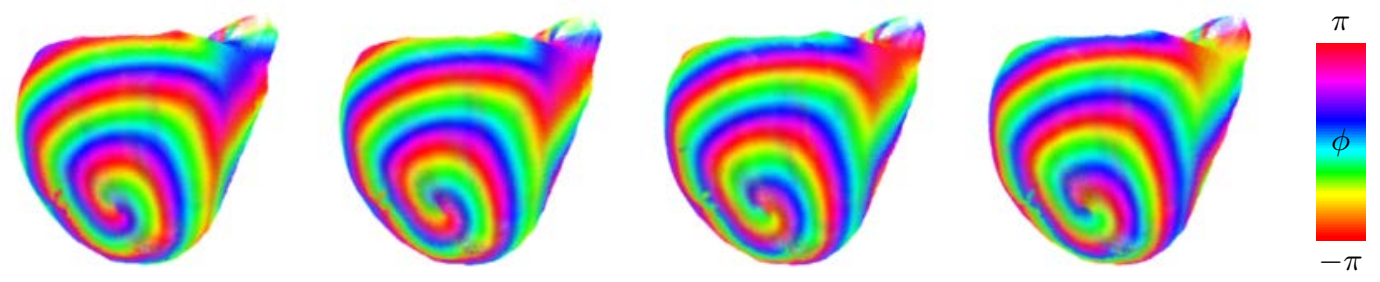

Figure 5.17: Phase representation $\phi_{u}$ of electrical scroll wave activity in left ventricular wall. Counter-clockwise rotating phase pattern with phase singularity depicting core region and rotational center of scroll wave. Compare with figure 5.6.
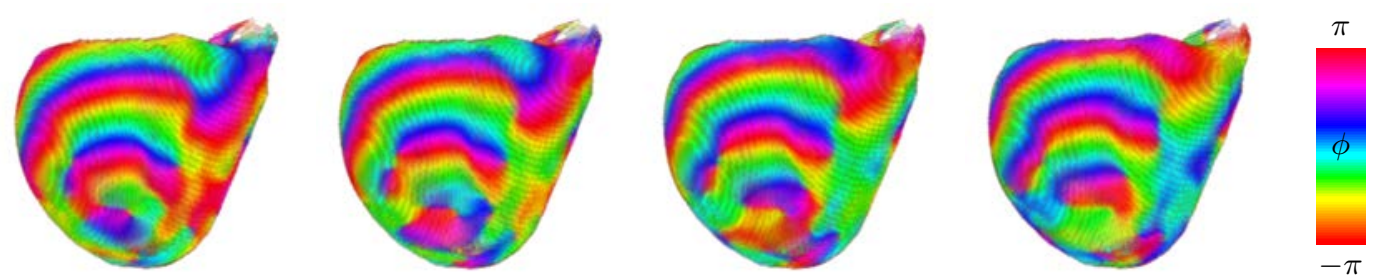

Figure 5.18: Phase representation $\phi_{E}$ of elasto-mechanical activity in left ventricular wall. Counter-clockwise rotating phase pattern with multiple phase singularities visible across ventricular surface. One persistent, re-occuring phase singularity nearby electrical phase singularity. Compare with figures 5.7 and 5.8 .

computed by introducing phase jumps $u(x, y, z, t)$ at the upstroke of the action potential in each time-series, that is for all $u_{x y z}(t)=0.1$ with $\partial_{t} u_{x y z}(t)>0$, and linearly interpolating all other phase values in between. The conversion of the electrical activity always automatically produced smooth and continuous phase fields. The phase representations of the patterns both exhibit similarly the rotational nature and topological structure of the underlying respective electrical or mechanical pattern and are both very similar. For instance, figures 5.17 and 5.18 show counter-clock-wise rotational patterns in the left ventricular wall due to electromechanical scroll wave activity. The mechanical pattern appears to be congruent with the electrical pattern. However, the circular symmetry is not always preserved, and in particular, seems to be disjunct or broken along horizontal directions. Both electrical and mechanical phase fields generally exhibit phase singularities or - within the volume - lines with converging phase, indicating organizational centers of the rotating activity, see next section. The detection and reconstruction technique presented in this section was not found to be robust in all situations, as the elasticity did not always exhibit the high degree of regularity that can be seen in figure 5.15. However, the post-processing techniques described above were effective in restoring the topological structure of the elasto-mechanical pattern to a good degree. Note that the technique in general does not require any presumptions or inverse computational techniques that rely on the overall tissue configuration or its properties but is based on a direct, local measurement to reconstruct electromechanical wave activity from deforming tissue.

\subsection{Electromechanical Scroll Vortex Wave Filaments}

In the previous sections, it was demonstrated that electrical and mechanical patterns of coupled electromechanical wave activity retain a similar appearance and underlying topological structure. The phase representations $\phi_{u}$ and $\phi_{E}$ of both time-varying patterns were shown to be highly similar, 


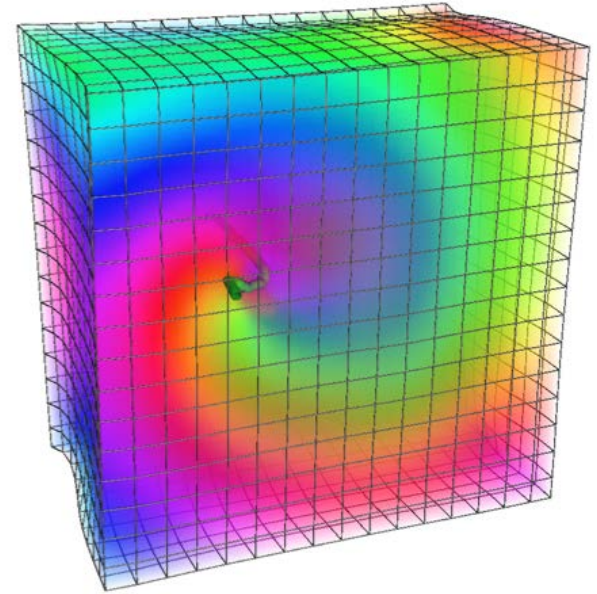

$\phi_{u}$

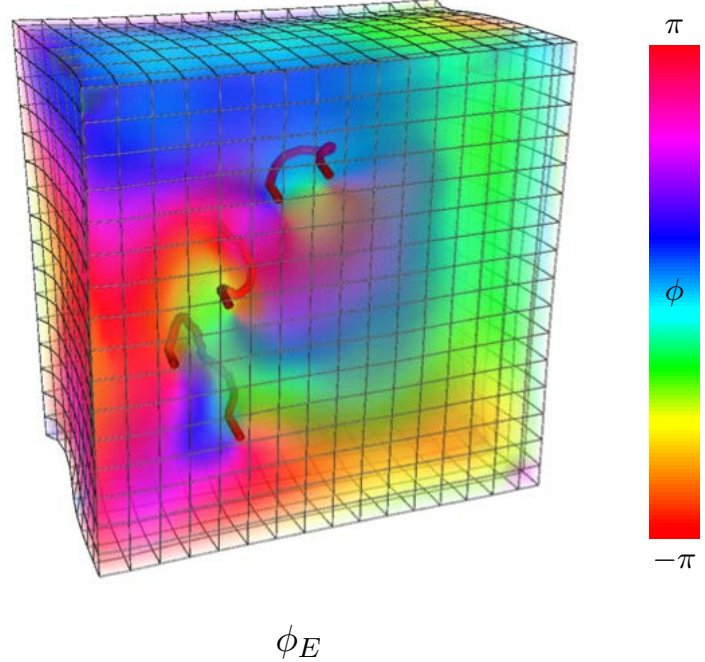

$\phi_{E}$

Figure 5.19: Three-dimensional time-varying phase fields $\phi_{u}$ and $\phi_{E}$ of electrical and elasto-mechanical activity in cubic-shaped elastic excitable bulk medium: Electrical (green line) and mechanical (red) filaments computed as topological defect lines or lines of phase singularity from time-varying three-dimensional phase fields. Similar rotational topological structure of the two phase fields. Data shown also in figure 5.9.

exhibiting congruent phase patterns and phase singular points. In three-dimensions, these singular points form lines of phase singularity. In the electrical phase representation $\phi_{u}$, these lines of phase singularity mark the rotational centers of scroll waves and correspond to their scroll vortex wave filaments. Lines of phase singularity that appear in the mechanical phase representation $\phi_{E}$ of deforming elastic excitable media, however, were not as yet described. Here, it is postulated that these lines of phase singularity relate to scroll wave vortex filaments while emerging as elasto-mechanical topological defect lines within the time-varying deformation patterns due to the rotational electromechanical activity. That is, the electromechanical activity expresses scroll vortex wave filaments within both the electrical as well as the mechanical picture. This section introduces the concept of electromechanical filaments and shows that they can be considered to be composed of entangled electrical scroll vortex wave filaments and elasto-mechanical topological defect lines.

\subsubsection{Detection and Reconstruction of Filament Structure}

Electrical and mechanical lines of phase singularity were reconstructed by analyzing the time-varying three-dimensional phase representations $\phi_{u}$ and $\phi_{E}$ of the electrical and mechanical activity. Similarly as described in sections 2.1.4 and 5.3.1, phase singular points were detected within the volume by computing the circular path integral around every site within the medium, integrating the gradient of the phase angle $\nabla \phi$ along the path integral. This was done by scanning, independently in each time step, the three-dimensional phase-field $\phi$ in three subsequent scans along the $x$-, $y$ - and $z$-axis in material coordinates respectively. Along each axis, the respective $x-y$-, $x-z$ - or $y-z$-planes were extracted consecutively to perform the calculations of the circular path integral for every site within each of the planes, see figure 5.20(a). In the two-dimensional planes, the integral was performed numerically within a $2 \times 2$ or $3 \times 3$ pixel neighbourhood, such that the sum consisted of either 4 or 8 summands. For every non-vanishing integral, the three-dimensional coordinate was stored together with an index in a list of detected phase singular points. As a result, a set of phase singular points 


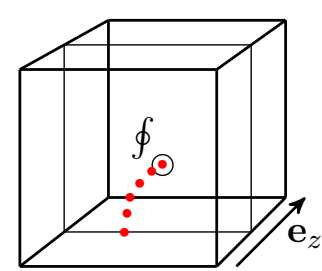

(a)

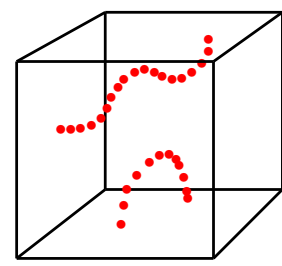

(b)

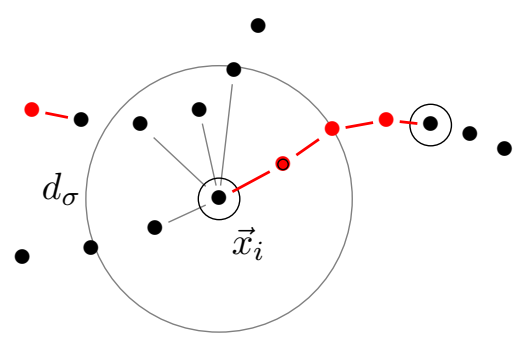

(c)

Figure 5.20: Reconstruction of three-dimensional filament structure: (a) medium is scanned in material frame using two-dimensional sampling cross-sections consecutively along all three principle $x-, y$ - and $z$ - orientations to extract in-plane phase videos for detection of phase singularities within each plane; detection involves performing circular path integral and storing $x-, y-, z$-coordinates for time step $t$ respectively (b) point cloud of detected phase singular points $\mathcal{P}^{s}$ at time $t$ (c) filamentlike structure of phase singular points connected to curvilinear lines or filaments

with three-dimensional material coordinates was obtained:

$$
\tilde{\mathcal{P}}^{s}=\left\{\vec{x}_{x, 1}^{s}, \ldots, \vec{x}_{x, K}^{s}, \vec{x}_{y, 1}^{s}, \ldots, \vec{x}_{y, L}^{s}, \vec{x}_{z, 1}^{s}, \ldots, \vec{x}_{z, M}^{s}\right\}
$$

with $K, L$ and $M$ detected points for each scan along the $x$-, $y$ - or $z$-direction respectively and $\vec{x}=(x, y, z)^{t}$ being an integer-valued material coordinate. Multiply detected phase singular points were removed from the list, such that a list of phase singular points:

$$
\mathcal{P}^{s}=\left\{\vec{x}_{x, 1}^{s}, \ldots, \vec{x}_{x, N}^{s}\right\}
$$

with $N \leq K+L+M$ was obtained. The list of points corresponded to a cloud of phase singular points in three-dimensional space. The cloud already retained a filament-like structure, see figure 5.20(b). The procedure was repeated for every time step and the electrical as well as mechanical data separately.

\section{Spatial Filament Reconstruction}

Typically, vortex filaments or topological defect lines are one-dimensional, arbitrarily shaped curves in three-dimensional space. Here, the sets of phase singular points were connected to curvilinear lines assuming an underlying filament-like structure, see figure 5.20(c). Euclidean pairwise distance matrices $\mathcal{M}^{d}$ of the distances among all phase singular points $\mathcal{P}^{s}$ measured in the Eulerian material frame were computed to derive a numerical scheme, with which points could be connected to curvilinear lines in space:

$$
\mathcal{M}^{d}(i, j)=\left|\vec{x}_{i}^{s}-\vec{x}_{j}^{s}\right|
$$

where each entry of the matrix stores the euclidean distance $d$ of the phase singular point with index $i$ to all other phase singular points with index $j$, where the diagonal entries with $i=j$ are undefined. The procedure was started by choosing a random point from the list of phase singular points and connecting it to its nearest neighbour to obtain a minimal filament consisting of two points that was consequently added to an empty set of filaments $\mathcal{L}$. The procedure was continued by computing 


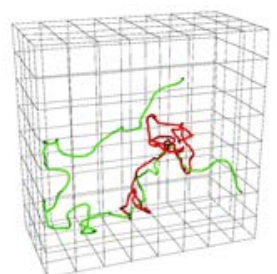

pair $\mathcal{L}_{1}^{e, m}$

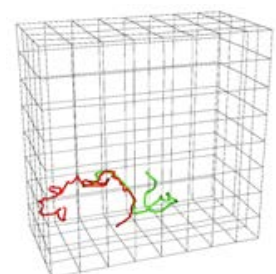

pair $\mathcal{L}_{2}^{e, m}$

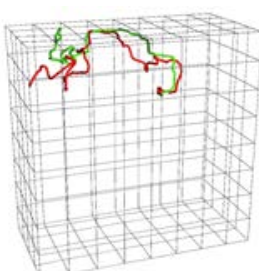

pair $\mathcal{L}_{3}^{e, m}$

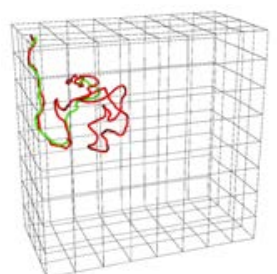

pair $\mathcal{L}_{4}^{e, m}$

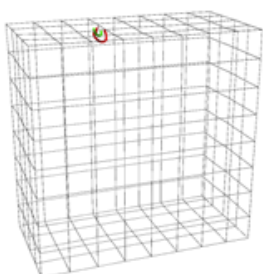

pair $\mathcal{L}_{5}^{e, m}$

Figure 5.21: Automatic pairwise association of co-localized electrical (green) and mechanical (red) filaments in space: pairs $\mathcal{L}_{i=1,2,3, \ldots}^{e, m}$ determined by minimization of sum of euclidean distances between points defining the filaments.

the distances from the end points of every filament $\mathcal{L}^{i}$ to all remaining phase singular points to determine the next nearest neighbouring points to add to the filament. However, the distances $d$ were constrained to be smaller then a lower threshold $d_{\sigma}$. If no point was within the sphere defined by $d_{\sigma}$, a new random point was chosen from the list of remaining phase singular points and a new minimal filament $\mathcal{L}^{i+1}$ was added to the set of filaments $\mathcal{L}$. During the following procedure, filaments could either grow or merge with other filaments or attach to the walls or close as a closed-loop. The procedure was performed iteratively, during which $d_{\sigma}$ could be adusted automatically, to construct a set of filaments and until all phase singular points were added to the set of filaments. The procedure took into consideration that filaments can take on the form of either closed-loops or that their ends attach to the boundary of the medium. Lastly, each material coordinate was replaced by the true Lagrangian world coordinate to visualize the filaments within the deforming medium.

The outcome is shown in figures 5.19, 5.22, 5.23 and 5.24. The procedure reconstructed reliably curvilinear lines from the phase singular point sets derived from the electrical as well as the mechanical phase field $\phi_{u}$ and $\phi_{E}$, also at higher filament densities during more turbulent activity as shown in figure 5.21. The filament-like lines were found, for the electrical as well as the mechanical case, to either exhibit closed-loops or attach with both their ends, perpendicularly aligned, to the boundaries of the medium. In the figures, electrical filaments are indicated in green and mechanical filaments are indicated in red. In general, the filaments could exhibit various lengths. However, the number, total length and density of mechanical filaments was always and consistently found to be larger than the number, total length and density of electrical filaments, $N_{m}>N_{e},|\mathcal{L}|_{m}>|\mathcal{L}|_{e}, \rho_{m}>\rho_{e}$.

\section{Co-localization of Electrical and Mechanical Filaments}

Two filaments from the electrical and the mechanical sets of filaments respectively could be associated with each other in space, see figure 5.21, by measuring the sum of minimal distances between all points defining the two filaments:

$$
\left|\mathcal{L}_{i j}\right|=\sum_{i=1}^{N_{e}} \sum_{j=1}^{N_{m}}\left|\vec{x}_{i}^{e}-\vec{x}_{j}^{m}\right|
$$

to obtain pairs of partially or fully co-localized electrical and mechanical filaments. In general, it was found that a partial number of the mechanical filaments $\tilde{N}_{m} \leq N_{m}$ was co-localized with or aligned in close proximity to electrical filaments and that electrical filaments were almost always accompanied by a nearby mechanical filament. This pairwise organization of electrical and mechanical 
filaments is here referred to as electromechanical filaments. The additional mechanical filaments did not seem to be directly related to electrical activity, see figures 5.23 and 5.24.

\section{Spatiotemporal Filament Tracking}

In addition to the spatial filament structure within each time step, the evolution of the curvilinear shapes of the filaments was tracked over time, to associate one filament at time step $t_{\tau}$ with its corresponding filament in the next time step $t_{\tau+1}$ to ultimately track the spatio-temporal evolution of the filaments, see figure 5.23. This allowed to measure distances of co-localized filaments over time, measure topological charge, vorticity and angular speed of the rotation of the outer phase field around one filament and to compare the lifetimes of electrical and mechanical filaments. Even though this methodology was not always necessary and applied because for most simulated situations the colocalization of filaments became obvious simply by visual inspection, see figure 5.23 , the possibility to measure rotational sense, angular speed of the surrounding phase field and co-localization of filaments became important during more complicated situations, as shown in figure 5.24.

\subsubsection{Properties of Electromechanical Filaments}

Figures 5.19, 5.22, 5.23 and 5.24 show the reconstructed scroll vortex wave filaments (green) and elasto-mechanical topological defect lines (red) during electromechanical scroll wave activity in a bulk as well as in a realistically heart-shaped elastic excitable medium. In both the bulk as well as the heart geometry it is possible to identify pairs of co-localized electrical and mechanical filaments which each can be associated with the rotational core region of a rotor. The electrical filament marks the rotational core of the electrical scroll wave and is commonly referred to as a scroll vortex wave filament. Here, it is found that its corresponding, co-localized mechanical filament exhibits the same vorticity and angular speed and can therefore be considered to similarly mark the rotational center and core region of the scroll wave. Throughout the simulations in the bulk, the pairwise co-localized electrical and mechanical filaments were found to be two closely related curves, see figure 5.23, often aligned in close proximity next to and wrapped around each other. For a wide range of situations, such as different electrical scroll wave shapes, fiber and electrical scroll wave filament tensions and orientations and relative orientations between scroll wave filament and fiber orientation, both filaments were entangled entities, which could be associated in pairs with each other in space and over time. The analysis also provides a better way to identify the organizational structure of the activity as compared to visual inspection and comparison of the two corresponding electrical and mechanical patterns, compare with figure 5.9. Multiple scroll waves show to be difficult to identify in the mechanical picture, whereas the display of the corresponding mechanical filaments reveals the approximate number and alignment of rotors in the bulk. The other mechanical filaments, that could not be associated directly with another electrical vortex filament, did not exhibit vorticity, and if the surrounding phase field rotated, then only by an angle smaller than $2 \pi$ followed by a, sometimes rapid, release-like counter-rotation. This effect was thought to indicate a release of elastic tension.

Co-localized electrical and mechanical filaments were also found in the heart-shaped medium, see figure 5.24. In the ventricles both the actual electrical and elasto-mechanical patterns as well as the corresponding phase representations exhibited equally rotor topology and rotational core regions. A double scroll wave rotor that was located with both counter-rotating rotors in the left ventricle produced two pairs of co-localized electrical and mechanical filaments. However, momentarily, the correlation of each pair appeared to be more complicated than a rigid pairwise, co-localized organi- 

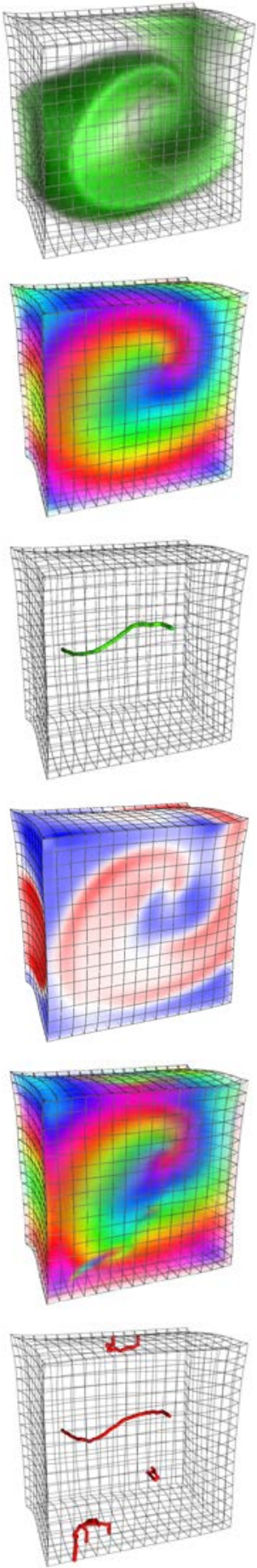
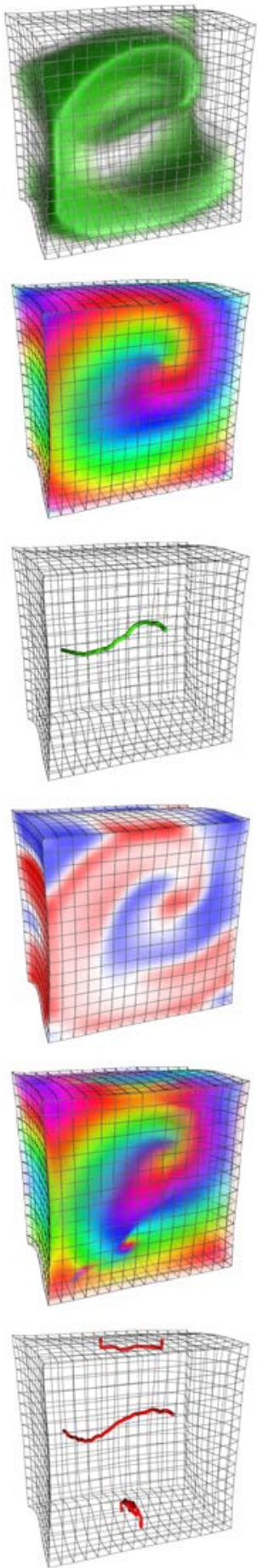
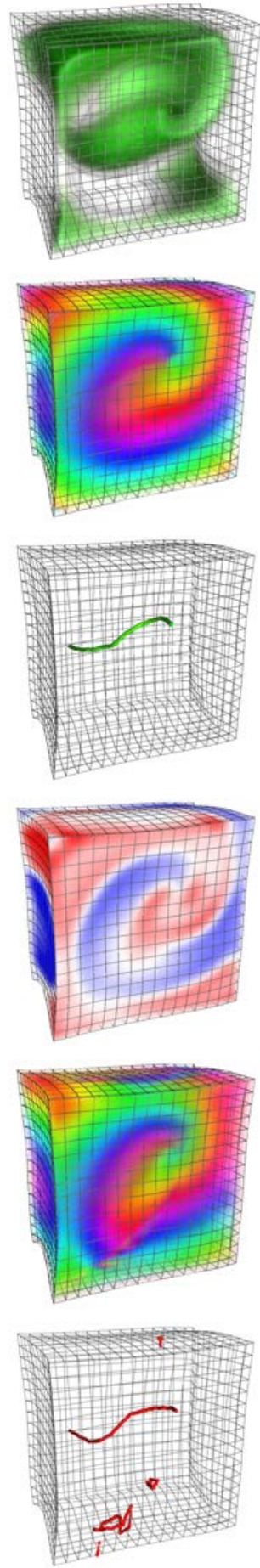

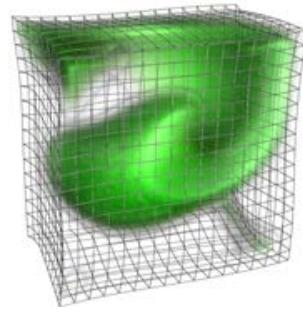

$20 \mathrm{mV}$
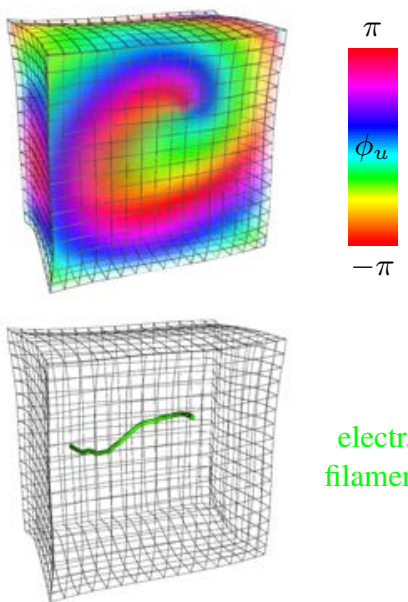

electr.

filament

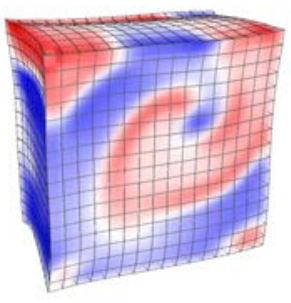

dil.
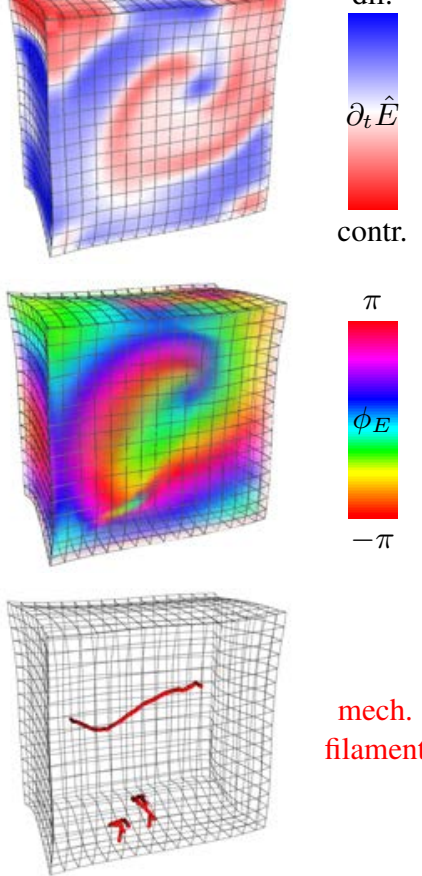

mech.

filament

Figure 5.22: Electromechanical scroll wave rotor rotating around electrical as well as mechanical filaments: the upper three panels show the electrical scroll wave rotor, its phase representation and the line of phase singularity computed from the phase representation, marking and equating to the electrical vortex filament. The lower three panels show the corresponding rotational elasto-mechanical rate of deformation pattern, its respective phase representation and the line of phase singularity computed from the phase representation, representing a topological defect line arising in the dynamic deformation pattern. Both lines of phase singularity are almost perfectly co-localized and both equally indicate the rotational center, vorticity and topology of the activity. 


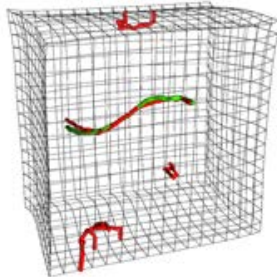

80

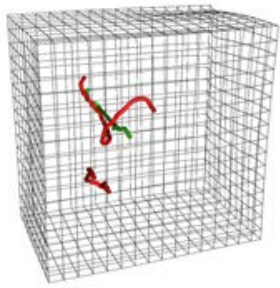

40

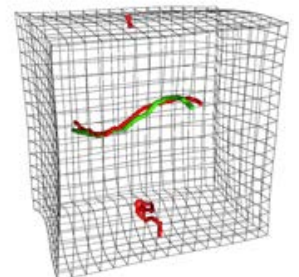

97

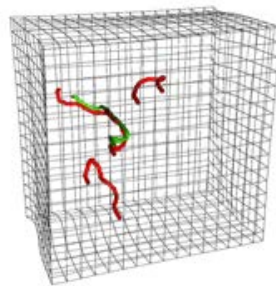

220

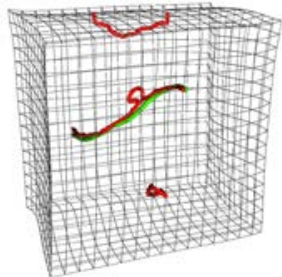

271

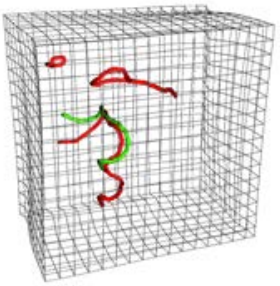

330

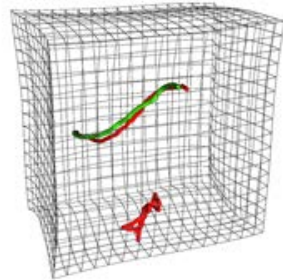

300

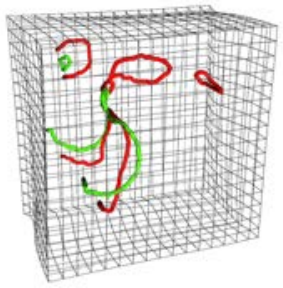

410
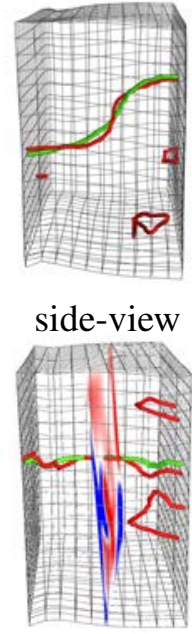

cross-section

Figure 5.23: Electromechanical filaments in deforming bulk-shaped elastic excitable medium with underlying scroll wave activity: electrical (green) filaments and mechanical (red) filaments. Upper sequence: filaments of single, stable rotor shown in figure 5.11. Lower sequence: filaments of wave breakup due to negative filament tension as shown in figure 5.9. Cross-sectional sampling plane throgh volume yields phase singular point within the in-plane mechanical pattern. This point equates to the intersection point of the mechanical filament with cross-section.
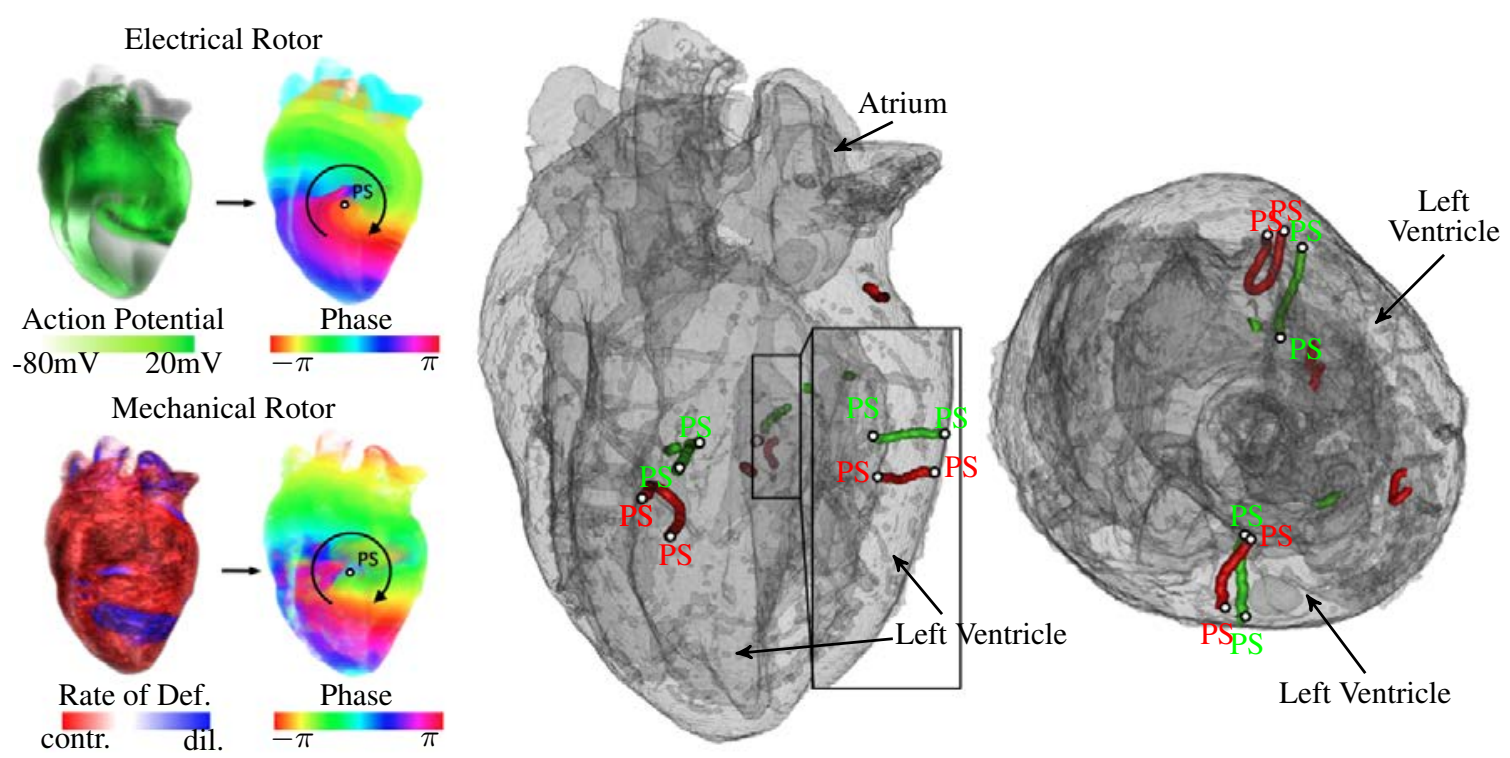

Figure 5.24: Electrical and mechanical filaments in ventricles of rabbit heart: electrical scroll wave (top left) and its phase representation exhibit rotational center (electrical phase singularity: PS). Likewise, the mechanical rate of deformation pattern (bottom left) and its phase representation exhibit rotational center (mechanical phase singularity: PS). Reconstruction of the filament-like structure of lines of phase singularity within the bulk of the ventricular muscle reveals pairs of co-localized electrical and mechanical filaments. The simulation suggests the existence of electro-mechanical filaments in the heart during tachycardia or fibrillation. Simulation on rabbit heart geometry. 
zation. The electrical vortex filaments showed to be accompanied by mechanical filaments intermittently, as seen in figure 5.24. More precisely, sometimes multiple mechanical filaments could loosely become entangled and disentangled with one electrical vortex filament throughout the duration of the simulation. This picture matches the dynamics of the phase representation pattern $\phi_{E}$, shown in figure 5.18, in which some phase singular points appear and disappear, while others persist and exhibit continuously vorticity with angular speeds matching the angular speed of the electrical rotational pattern. This behavior, the break-up of the bound, pairwise organization and transition towards a less correlated, more dynamic structural organization of electromechanical filaments, may be caused by the underlying muscle fiber anisotropy, but it may also be a characteristic feature of coupled electromechanical scroll wave activity in general. Presumably, the unassociated mechanical filaments are related to the passive elastic response of the tissue, however, the substance of these filaments remains unclear. The procedure used to reconstruct the isochronal structure of the wave pattern may have revealed these lines to be passive elastic nodal lines. However, they may also have been caused - at least in parts - by the reconstruction procedure itself. Overall, the simulations suggest the existence of electromechanical filaments within the heart during cardiac tachyarrhythmias.

The analysis presented in this and the previous sections shows that mechanical deformation can reveal important information about the topology and structural, three-dimensional organization of the underlying electrical activity. This has important implications with regard to the experiments described in chapter 6.

\subsubsection{Emulation of Imaging Experiment}

The coupled electromechanical simulations make it possible to emulate the imaging experiment that is described in chapter 6. Figure 5.25 shows the break-up of one clock-wise rotating scroll vortex wave (green) due to negative filament tension. The imaging situation is mimicked by the introduction of a cross-sectional plane at midwall. This plane simulates a cross-sectional imaging plane of a two-dimensional tomographic imaging modality that intersects the heart wall and captures its deformations, see also experimental design in chapter 6 . The cross-sectional plane indicates the volumetric strain-rate that was subsampled using the cross-section from the underlying three-dimensional pattern. However, the corresponding two-dimensional strain-rate pattern, that is computed from deformations visible only within the plane, was found to be very similar. Dilating and contracting strain-rates are indicated in blue and red respectively, as described in the previous sections. Here, the scroll wave break-up can be observed within the entire bulk due to the visualization that renders parts of the volume transparent. However, in a real experiment, the electrical activity could only be imaged on the surface using optical techniques. The intramural activity could be imaged, following the ideas and concepts discussed in the previous sections, within the cross-section. Here, the elasto-mechanical pattern at midwall exhibits a rotational spiral wave-like pattern. Overall, the scroll wave experiences a torsional twist that is caused by faster propagation speeds of the electrical activity and wavefront closer to the boundaries of the medium and slower propagation speeds at midwall. As a result, the electrical vortex wave filament begins to coil with the coiling spreading from the surface towards the inner volume. At the same time, the filament length increases. This increase is referred to as negative filament tension. Negative filament tension is associated with wave break-up and the break-up mechanism is hypothesized to underlie the emergence of spatial-temporal chaos in the heart. The coiling filament, increasing in size and length, eventually interacts with itself, which leads to the break-up of the filament and the creation of another new filament close to the surface. In other terms, close to the surface the scroll wave encroaches on its own refractory tail and breaks up into two scroll waves, see 

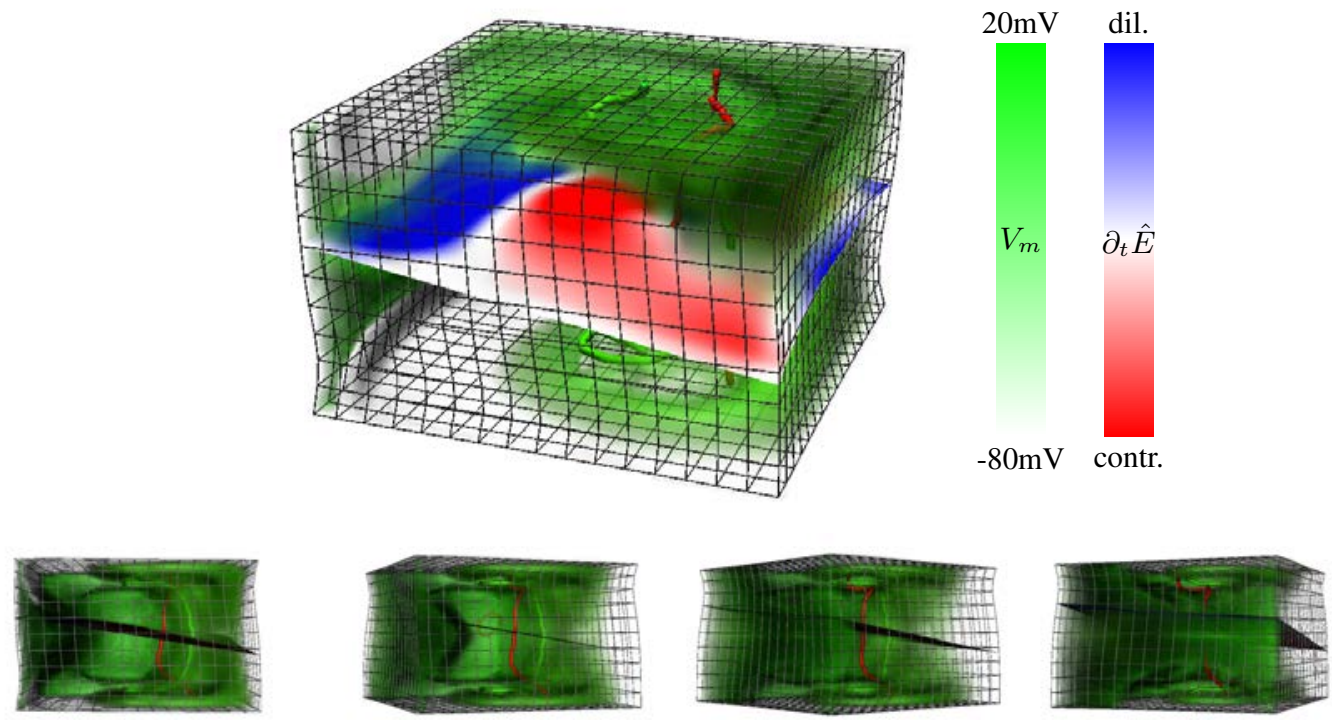

Figure 5.25: Break-up of scroll vortex wave (green) due to negative filament tension: simulation of imaging experiment described in chapter 6 with cross-sectional imaging plane placed at midwall, indicating spiraling elasto-mechanical activity with dilating (blue) and contracting (red) rates of deformation. The electromechanical pattern of the scroll wave rotor reveals a stable rotor at midwall, whereas the activity observed on the surface exhibits wave break. Electrical (green) filament start to coil at the boundaries, which induces the break-up. Mechanical (red) filament evolves accordingly.

also figure 5.9. However, meanwhile, at midwall, the rotor remains stable. Both electrical (green) and mechanical (red) filaments evolve correspondingly. They remain straight lines within the bulk, are aligned in parallel, exhibit the same vorticity and both lead towards the upper as well as the lower wall. Here, the upper and lower walls are supposed to correspond to the epi- and endocardial walls. Within the cross-section, the rotating elasto-mechanical deformation pattern would reveal the stably rotating scroll wave at midwall. Hence, the simulation demonstrates that we would, in this particular situation and for a short time, be able to observe two fundamentally different patterns on the surface and inside the wall: the emergence of chaos on the surface and a stable rotor inside the wall. It can only be speculated whether the here observed behavior relates to real scroll wave break-up in the heart. However, the example shows that imaging of the intramural wave activity could significantly contribute to a better understanding of cardiac fibrillation.

\subsection{Inverse and Electromechanical Wave Imaging}

The contractile motion and deformation of the heart muscle needs to be captured post-acquisition, that is after the imaging and acquisition process, during the analysis and post-processing of the acquired data, using computational techniques. Typically, this is done using image registration and motion tracking techniques, see section 3.4 in chapter 3. Here, it is investigated how the ideas and concepts presented in the previous sections can be combined with computational techniques to register deformations. This section presents and discusses motion registration and deformation tracking techniques developed for the analysis of experimental motion and deformation data that was acquired 

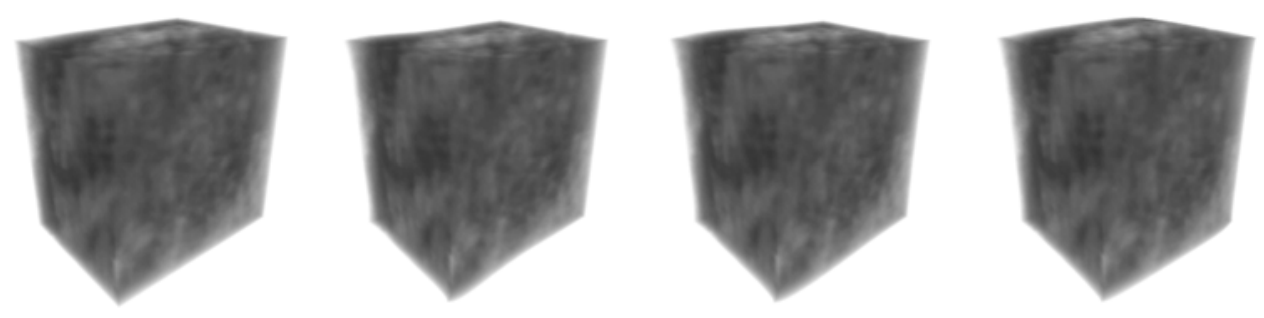

Figure 5.26: Synthetic deformation of speckle image volume data showing part of a rabbit ventricular wall obtained from $\mu$-CT scan: deformation according to planar electromechanical wave propagation, see figure 5.3. Inverse electromechanical wave imaging or reconstruction of three-dimensinal electromechanical wave patterns in synthetic data mimicking real experimental data. Resampling in voxel geometry allows to create synthetic deformation data that can be analyzed using spatial correlation algorithms.

in this work, see chapter 6 . At the end of this section, the techniques are presented to be able to detect a spiral wave and its respective spiral wave core in experimental deformation data of cardiac cell culture tissue preparations.

\section{Electromechanical Wave Imaging}

The term electromechanical wave imaging was introduced by Konofagou and co-workers. ${ }^{205,206,215}$ In their work, they demonstrated that the onset of contraction within the ventricular muscle, imaged at high-speeds using ultrasound, follows paths that are known to be conduction pathways of electrical activity. Consequently, they provided visualizations of spread of mechanical activation throughout the heart and were able to distinguish activation patterns of cardiac arrhythmia, such as for instance atrio-ventricular dissociation, from periodic sinus activity. However, their use of the term electromechanical wave imaging refers to an experimental measuring technique that is solely based on ultrasound elastography imaging and does not measure both electrical and mechanical activity simultaneously. Here, electromechanical wave propagation is similarly understood to be characterized by the onset of contraction, see previous sections, and it is postulated that imaging of electromechanical wave propagation is feasible if targeting dynamic features, such as contractile motion and rates of deformation, of the elasto-mechanical activity of the heart. However, it is also understood that electromechanical wave propagation is additionally composed of electrical activity and that both closely coupled patterns need to be studied and imaged. Earlier, it was postulated by Otani and co-workers ${ }^{192}$ that the use of ultrasound together with an inverse mathematical model, may provide visualizations of action potential wave propagation in the heart. The approach is different from the one described above in that it analyzes the static deformation of the cardiac muscle using an inverse model involving constitutive laws of an elastic continuum body, its mechanical and underlying fiber configuration and an overdetermined system of equations to derive spatial distributions of active stress within the tissue, that is ultimately directly related to electrical action potential wave activity. Hence, here, electromechanical wave imaging is also referred to as being related to inverse imaging. The techniques presented in this section aim to bridge the gap between the two concepts provided by Konofagou and Otani. 


\section{Three-dimensional Electromechanical Wave Reconstruction}

The reconstruction of the three-dimensional electromechanical wave pattern, that was described in the previous sections of this chapter, was possible because the simulations provided the complete deformation data at all times. It was possible to track the location of every single tissue element through space and over time, as it was possible to request the coordinates of the material particles at runtime or after the completion of the simulations. Other quantities, such as strains or strain-rates could be requested or derived from the data easily as well. Generally, and in particular in experiments, however, this data is not readily available and is required to be extracted from the imaging data before it can be used in the following reconstruction procedure. For the reconstruction of three-dimensional electromechanical wave patterns, it becomes necessary to capture and analyze tissue motion within volumetric, tomographically acquired data and to reconstruct the three-dimensional deformation itself before further analysis can be done.

Figure 5.26 shows a deforming cubic-shaped bulk medium consisting of speckle image volume data that shows parts of the ventricular wall of a rabbit heart. The data was acquired using computerized tomography, see section 4.4, and used here together with simulated deformation data to produce synthetic data that mimicks experimental data of three-dimensional tissue-motion. Here, every voxel of the volumetric $\mu$-CT-data was assigned to one hexaedral cell of the simulation shown in figure 5.3. Each voxel was then deformed and moved in space according to the simulation. Here, the underlying planar electromechanical wave activity inside the bulk with uniformly linearly transverse aligned muscle fibers causes a horizontal contraction, see section 5.2. Using voxelization techniques, one could resample the deformed voxels in each time step into a three-dimensional regular voxel-lattice image structure, to create three-dimensional time-varying image data of tissue motion. Nowadays, no imaging modality is capable of producing such data sets. Three-dimensional ultrasound, for instance, is capable of acquiring up to about $\sim 20$ volumetric frames per second. To image the fast transient behavior of spiral or scroll waves, which typically rotate at frequencies of $f_{\text {rot }} \sim 10-20 \mathrm{~Hz}$, the acquisition speed is insufficient to resolve the three-dimensional pattern appropriately. In principle, the methodology discussed above is able to provide an in-silico testbed for three-dimensional electromechanical wave pattern reconstruction. However, the work remained incomplete and is only described here to portray and highlight the potential of the analysis imaging described in this chapter. The following sections describe tracking of deformation in two dimensions. However, the technique can easily be extended to be used for the reconstruction of three-dimensional deformation patterns.

\subsubsection{Elastic Deformation Tracking}

A computational elastic deformation tracking technique was developed, to track time-varying elastic deformations inside movies or image sequences showing actively contracting cardiac tissue. The technique employs a combination of motion and deformation tracking and regularization of the deformation using an elastic computational model. The technique had to be able to handle various types of experimental data with different propeperties, see chapter 6. Motion and deformation data was obtained from either ultrasound or fluorescence imaging. The ultrasound imaging produced speckle image movies showing the contracting heart wall at high spatial resolutions. Fluorescence imaging produced movies showing the moving and contracting heart surface at low spatial resolutions. Other optical imaging produced movies showing the contractile activity of cardiac cell cultures, see section 5.5.3. While the ultrasound data consisted of noisy speckle image data without features, the fluorescence data included significant intensity fluctuations due to the emission of fluorescence. The 
extraction of motion inside these data sets was achieved using spatial correlation algorithms, see below, and the deforming tissue configuration was reconstructed using the elastic model, see also below, in order to be able to perform the electromechanical wave pattern reconstruction procedure described in the previous sections in this chapter, see chapter 6 . In principle, the involvement of the elastic computational model allows to introduce an inverse mathematical model as described previously ${ }^{192}$ for the reconstruction of electrical activity.

\section{Spatial Correlation Analysis and Motion Tracking}

Tissue motion was tracked using spatial correlation algorithms, see also section 3.4. The motion of the tissue was considered to correspond to the optical flow of intensity grayvalues that occurs in between two images showing the same piece of tissue at two times $t$ and $t^{\prime}$. When the tissue moved in between the two times in which the two images were acquired, the overall grayvalue pattern should have experienced a shift or deformation accordingly. Consequently, it becomes possible to compute the optical flow in between the two images by comparing small local subregions $\mathcal{S}_{x y}^{t}$ and $\mathcal{S}_{\Delta x \Delta y}^{t^{\prime}}$, one located at the coordinate $(x, y)$ in the first image $I(x, y, t)$, the other one located at various positions $(x+\Delta x, y+\Delta y)$ around that coordinate in the other image $I\left(x, y, t^{\prime}\right)$, and finding the offset $(\Delta x, \Delta y)$ that maximizes the degree of similarity between the two subregions. In other words, for various locations within the image, each surrounding pattern of grayvalues is analyzed, to find the two matching patterns that correspond to the same piece of tissue and that can be determined to be correlated over space and time. The offset $(\Delta x, \Delta y)$ between these two spatially correlated patterns corresponds to the tissue displacement $\vec{u}_{x y}^{t}$ at the location $(x, y)$ measured in pixels at time $t$. In particular, the scanning of the neighbourhood is conducted within an interrogation window $\mathcal{I}$ around the coordinate $(x, y)$, which is typically chosen to be larger than the expected average magnitude of the displacement. Figure 5.27(a) shows a grid of black nodes placed on top of a grayvalue image. Every node corresponds to one location $(x, y)$, at which the spatial correlation tracking is performed. The entire grid of nodes constitutes a tracking grid and deriving the displacements $\vec{u}_{x y}^{t}$ for every node corresponds to deriving the optical flow from time step $t$ to $t^{\prime}$. Generally, the grid does not have to retain a regular lattice structure and can also have arbitrary shapes, see figure 6.12 in chapter 6 . The small white rectangle centered around the node $(i, j)$ with coordinate $(x, y)$ is the subregion $\mathcal{S}_{x y}^{t}$. Typically, its size ranges from $5 \times 5$ to up to $50 \times 50$ pixels and it can also be circularly-shaped. The large white rectangle is the interrogation window $\mathcal{I}$ within which the search is conducted. The other small white rectangles are supposed to indicate the sweeping of the subregion $\mathcal{S}_{\Delta x \Delta y}^{t^{\prime}}$ over various offsets $(x+\Delta x, y+\Delta y)$ through the interrogation window, following a scanning scheme along a twodimensional raster through the window. The red rectangle indicates the location $(x+\Delta x, y+\Delta y)$ in the other image at time $t^{\prime}$ for which the highest degree of similarity between the two subregions was found. Correspondingly the black vector indicates the displacement $\vec{u}_{x y}^{t}$ at node $(i, j)$ positioned at the coordinate $(x, y)$.

A commonly used, very robust similarity measure is the mean quadratic difference $\mathcal{D}$ of intensity grayvalues:

$$
\begin{aligned}
\mathcal{D}(\Delta x, \Delta y) & =\frac{1}{N \cdot M} \sum_{x=1}^{N} \sum_{y=1}^{M}\left|I(x, y)-I^{\prime}(x+\Delta x, y+\Delta y)\right| \\
& =\left|\mathcal{S}_{x y}^{t}-\mathcal{S}_{\Delta x \Delta y}^{t^{\prime}}\right|
\end{aligned}
$$




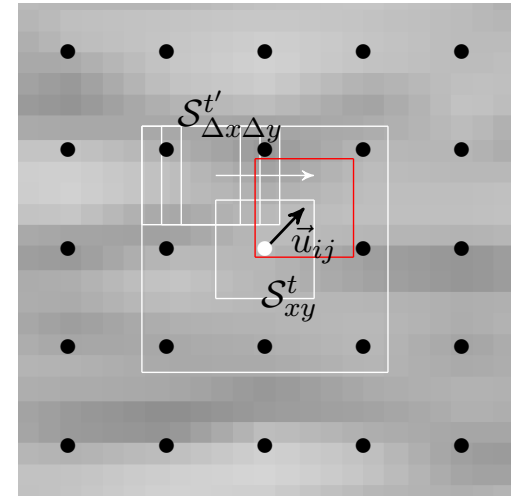

(a)

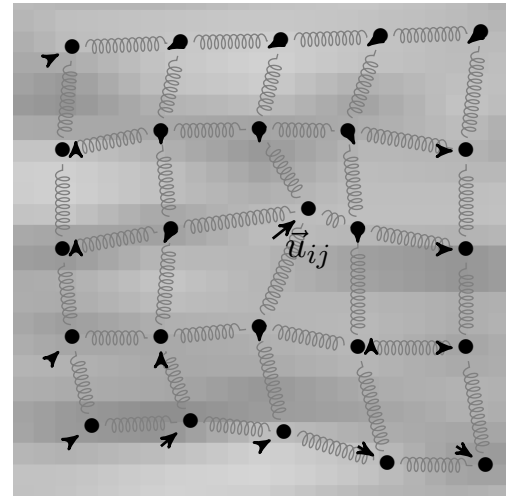

(b)

Figure 5.27: Elastic motion registration used to track tissue motion: Combination of motion tracking algorithm and elastic computer model for regularization of tracking to maintain affine, smooth deformation of continuum body. Tracking grid is simultaneously an elastic mass-spring system that introduces forces between nodes of the tracking grid. This ensures preservation of topology of configuration of the continuum body and simultaneously compensates outliers. (a) undeformed reference configuration $\chi_{0}$ of tracking grid at $t=0$ on grayscale image showing moving tissue (b)

which is computed using the sum of differences of all respective pairs of pixels in the two subregions. The mean quadratic difference vanishes if comparing two subregions that show the exact same pattern and is found otherwise to be minimal at the location $(x+\Delta x, y+\Delta y)$ within the plane that is scanned as described above. When the procedure is repeated for every black node on the grid, the spatial correlation and motion tracking technique provides the displacement field between the two video frames. The displacements of all nodes together provide the deformation of the node configuration, which equates in the case of this measurement technique to the tisssue configuration. The resolution of the tracking grid can correspond to the image resolution, which equates to placing nodes located at every pixel, or it can be corrrespondingly smaller, placing nodes at a distance separated by a few pixels from each other by a distance $w_{x / y}$ :

$$
\Pi_{\chi}(i, j)=\left(\Delta w_{x} i, \Delta w_{y} j\right)
$$

The analysis assumes that the optical flow occurs within the imaging plane and that, in particular for the case of cross-sectional imaging, the evolving pattern does not exhibit completely uncorrelated morphologies in between frames, as it would be the case for out-of-plane motion.

\section{Inter-Frame Tracking and Frame of Reference}

Repeatedly applying the motion tracking described above and collecting the subsequent displacements $\vec{u}_{i j}^{0}, \vec{u}_{i j}^{1}, \ldots, \vec{u}_{i j}^{t-1}, \vec{u}_{i j}^{t}$ of each node and adding them to the previous displacements results in the actual position $\vec{x}_{i j}^{t}=\vec{x}_{i j}^{0}+\vec{u}_{i j}^{0}+\vec{u}_{i j}^{1}+\ldots+\vec{u}_{i j}^{t-1}+\vec{u}_{i j}^{t}$ and a trajectory $\mathcal{T}_{i j}=\left\{\vec{x}_{i j}^{0}, \vec{x}_{i j}^{1}, \ldots, \vec{x}_{i j}^{t}\right\}$ of the node. The trajectories of all nodes together constitute the mechanical deformation of the configuration $\chi$ of nodes, which can be thought of to resemble the deformation of the tracked tissue. 


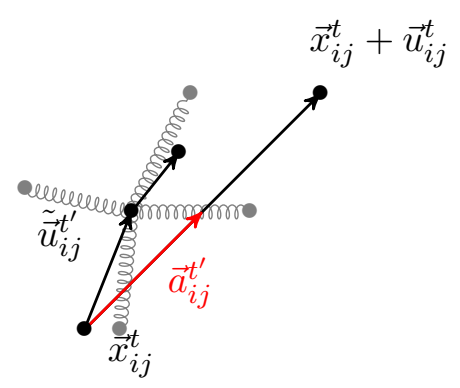

(a)

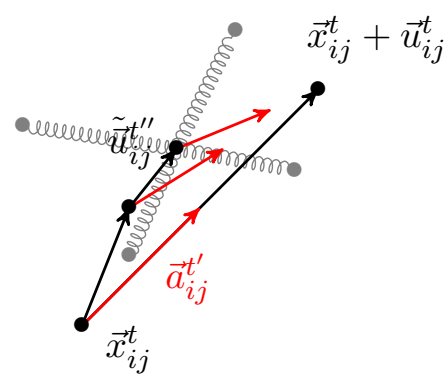

(b)

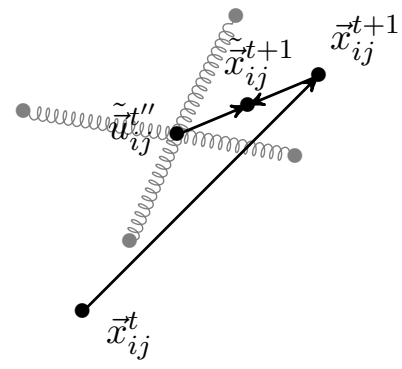

(c)

Figure 5.28: Scheme of elastic deformation tracking with coupled Eulerian motion tracking and elastic particle system regularizing the raw displacements $\vec{u}_{i j}^{t}$ and configuration $\chi_{\vec{u}}^{t}$ that are output of the motion registration procedure. The elastic particle system evolves dynamically towards the tracked configuration $\chi_{\vec{u}}^{t}$, however, smoothes the configuration $\chi_{\vec{u}}^{t}$

Here, two tracking modes were used, depending on the imaging situation, see chapter 6 . During inter-frame tracking, two subsequent frames or subsequent frames with a fixed distance of two or more frames in between were compared to each other to compute the inter-frame displacements. In this tracking mode, the tracking grid deforms continuously and the tracking is started in the next time step with each node being positioned at a new coordinate $\vec{x}_{i j}^{t+1}=\vec{x}_{i j}^{t}+\vec{u}_{i j}^{t}$ given by the last position and the last tracked displacement at time $t$. The trajectory for each node of the grid is produced as described above and the trajectory similarly describes the motion of the node as well as the motion of the material particle that is tracked. In particular, in this tracking mode, errors in the tracking sum up over time. The tracking mode is also referred to as Eulerian tracking as the motion is tracked in material coordinates of the tracking grid. In contrast, during Lagrangian tracking, the image data was tracked with respect to one fixed reference frame that was chosen before the tracking procedure. In this tracking mode, the tracking grid remains undeformed and the trajectory of each tracked material particle becomes $\mathcal{T}_{i j}=\left\{\vec{x}_{i j}^{0}, \vec{x}_{i j}^{0}+\vec{u}_{i j}^{1}, \ldots, \vec{x}_{i j}^{0}+\vec{u}_{i j}^{t}\right\}$. In particular, the nodes of the tracking grid do not correspond to the tissue configuration as above. In this tracking mode, errors of the tracking do not sum up over time. However, the trajectory of each material particle of the tissue may not be smooth anymore due to deviations from tracked displacements of one to the next time step. Most of the time, the inter-frame tracking was used. Note that the tracking can only be performed within the inside of the image within a certain range away from the boundaries.

\section{Elastic Deformation Tracking}

Figure 5.27(b) shows the displacement vector field that is obtained when applying the spatial correlation tracking described above repeatedly for every node of the tracking grid after one time-step. In addition to the displacement vectors, the figure depicts springs which connect every node with its nearest neighbouring nodes. These springs are supposed to indicate that the nodes of the tracking grid are part of an elastic particle system, as described in sections 4.2 in chapter 4 . The elastic particle system regularizes the displacements by imposing elastic forces in between the nodes to have the tracking grid to behave like an elastic continuum body. Instead of displacing every node by its raw registered displacement vector $\vec{u}_{i j}^{t}$, the grid is deformed dynamically in an iterative procedure towards the configuration $\chi_{\vec{u}}$ that is given by the raw registered displacements $\vec{u}_{i j}$. Figure 5.28(a-c) 
shows a sketch that illustrates this scheme. At time $t$ the motion registration computed the displacement $\vec{u}_{i j}^{t}$ for the node $(i, j)$, which is positioned at $\vec{x}_{i j}^{t}$. The particle $(i, j)$ is accelerated, indicated by the red arrow, towards the position $\vec{x}_{i j}^{t+1}=\vec{x}_{i j}^{t}+\vec{u}_{i j}^{t}$, however, at a rate $\vec{a}_{i j}^{t^{\prime}}$ that requires many iterations of the equation of motion in order for the particle to get to the position. Meanwhile, the particle experiences the elastic forces from its surrounding, indicated by springs, and its trajectory gets altered accordingly, indicated by the displacement vectors $\tilde{\vec{u}}_{i j}^{\prime}$ and $\tilde{\vec{u}}_{i j}^{t^{\prime \prime}}$. The indices $t^{\prime}, t^{\prime \prime}, \ldots$ indicate the steps during the iterative procedure. Particle systems are dynamic systems, which allow waves and vibrations to find an equilibirum state by distributing forces through the elastic system and minimizing its energy accordingly, see section 2.2.5. The idea of the elastic regularization was to maintain a smooth, continuous deformation of the continuum body and to reduce the influence of erratically tracked displacements. Eratically tracked displacements, outliers, are smoothed as each displacement occurs in accordance with the displacements of the surrounding continuum, which effectively constrains the motion of each particle. Each iteration, the elastic particle system uses the position $\vec{x}_{i j}^{t}+\vec{u}_{i j}^{t}$ as a reference position to which the system is supposed to converge. Accordingly, the particles get accelerated towards these positions in each iteration, indicated by red arrows in figure 5.28(b). The position of each particle or node at the end of the iterative procedure yields:

$$
\tilde{\vec{x}}_{i j}^{t}=\vec{x}_{i j}^{t}+\tilde{\vec{u}}_{i j}^{t^{\prime}}+\tilde{\vec{u}}_{i j}^{t^{\prime \prime}}+, \ldots
$$

This position is weighted with the raw registered position $\vec{x}_{i j}^{t}+\vec{u}_{i j}$ :

$$
\tilde{\vec{x}}_{i j}^{t+1}=\frac{\vec{x}_{i j}^{t}+\vec{u}_{i j}^{t}+\tilde{\vec{x}}_{i j}^{t}}{2}
$$

to find an approximation $\tilde{\vec{x}}_{i j}^{t+1}$ of the position $\vec{x}^{t+1}=\vec{x}_{i j}^{t}+\vec{u}_{i j}$ considering the continuous affine, elastic deformation of the tissue. The point $\tilde{\vec{x}}_{i j}^{t+1}$ is assigned to each node in the next time step. Accordingly, the new configuration $\chi_{\tilde{\vec{u}}}$ of the particles and nodes of the tracking grid is a smoothed version of the configuration $\chi_{\vec{u}}$ that was given by the initial registration. The elastic particle system changes each trajectory $\mathcal{T}_{i j}$ of each particle to a smoothed trajectory $\tilde{\mathcal{T}}_{i j}$. The elastic deformation tracking scheme was found to effectively remove ripples and tearing of the tracking grid. In particluar, the scheme was found to be able to track tissue configurations within noisy ultrasound speckle movie data.

\subsubsection{Elastic Deformation Tracking tested with Synthetic Data}

The elastic deformation tracking scheme described in the previous section was tested with synthetic deformation data that was created using the computational model described in this thesis, see previous sections and chapter 4. Figure 5.29 shows a simulation of coupled electromechanical spiral wave activity in a two-dimensional elastic excitable medium, see also figure 5.6. In addition to the original elastic particle system, denoted as system 1, that is part of the coupled electromechanical simulation, a second particle system, denoted as system 2, was used to track and capture the deformation of the first system of the coupled electromechanical simulation. Panel (a) in figure 5.29 shows the spiraling electrical activity (green) in system 1. Panel (b) shows the according deformation and spiraling strain-rate pattern with dilating (blue) and contracting (red) rates of deformation in system 1. Panel (c) shows system 2 that was deformed to match the actual configuration $\chi$ of system 1, which was accordingly provided as input to system 2 , however, after having added random small displacements to the positions of each node $\vec{x}_{\eta}=\vec{x}+\vec{u}_{\eta}$. All nodes of system 2 were accordingly displaced 


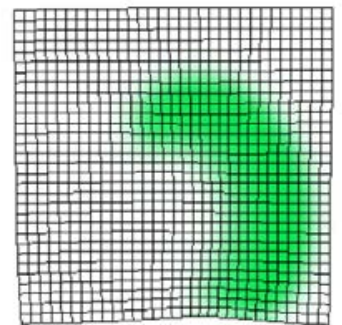

(a)

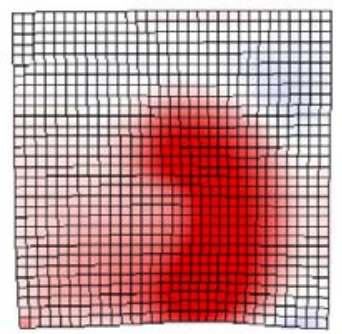

(e)

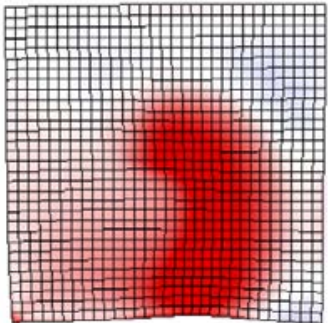

(b)

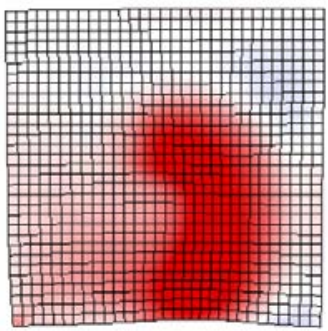

(f)

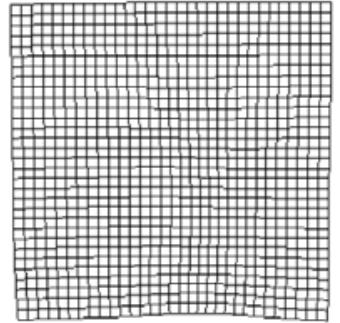

(c)

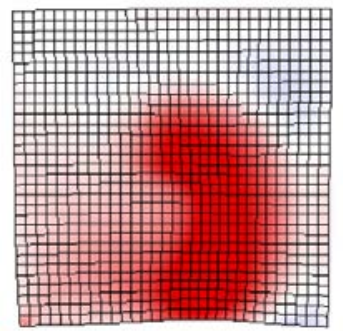

(g)

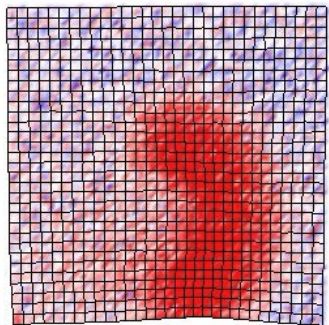

(d)

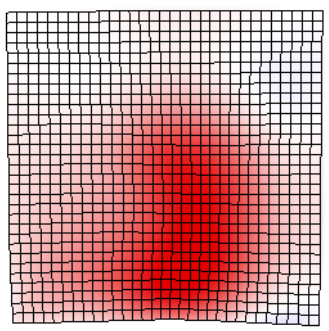

(h)

Figure 5.29: Elastic deformation tracking tested with synthetic data: deformed configurations produced with coupled electromechanical simulation of spiraling wave activity with (a) electrical spiral wave pattern (green) and (b) according elasto-mechanical deformation with dilating (blue) and contracting (red) rates of deformation patterns. Panels (a-b) show evolution of system 1. System 1 provides mechanical configurations that are suposed to be tracked by system 2 . Panels (c-h) show evolution of system 2 . System 2 receives the mechanical configuration of system 1 plus a noisy, random displacement of every node. Accordingly, panel (d) shows a noisy strain-rate pattern. Panels (e-h) show system 2 converging to the noisy configuration $\chi_{\tilde{u}}$ shown in (c-d), employing the elastic deformation tracking techniques described in the previous sections. The elastic deformation tracking smoothes the tracking. Good qualitative agreement of original and mimicked deformation.

throughout the evolution of system 1 directly to the positions of the input that was provided. The noise was introduced assuming that tracking of experimental image data would introduce noise to the tracked displacement vector field. The noise was scaled to lie within a fractional distance to the next nearest-neighbouring node. Panel (d) shows the same configuration as shown in panel (c), but additionally indicates the strain-rate as described previously. Accordingly, the pattern as well as its spatial-temporal evolution appears to be highly noisy. The following panels show different deformed configurations of system 2 , which were retrieved by converging system 2 to the configuration shown in panels (c-d), employing the same elastic deformation tracking techniques described in the previous sections, yielding different approximate configurations $\chi_{\tilde{u}}$ for different tracking parameters and configurations of the tracking. All panels in the lower row show the a smoothed version of the configuration shown in panels (c-d), demonstrating that the elastic deformation tracking technique can be used to inhibit noise that may arise in the analysis of data obtained in imaging experiments. Nevertheless, even though the images indicate that the approximated versions of the configuration deviate substantially from the original configuration from system 1, all images show a spiraling elasto-mechanical pattern and thus demonstrate that the technique can be used to detect spiraling or rotating patterns qualitatively. The elastic deformation tracking technique showed to be very effective in tracking the deformation inside noisy ultrasound speckle image movies. 

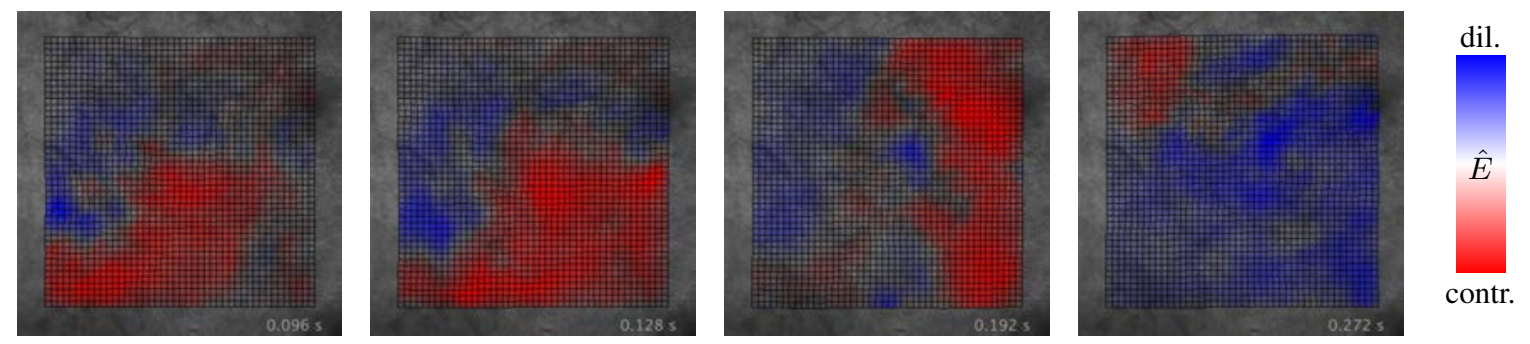

Figure 5.30: Elasto-mechanical spiral wave rotor in engineered cardiac cell culture based on a collagen scaffolding extracellular matrix and cardiomyocte mix that allows the substrate to undergoe large deformations: spiraling contractile activity and deformation (strain) occured continuously around almost stationary rotational core.
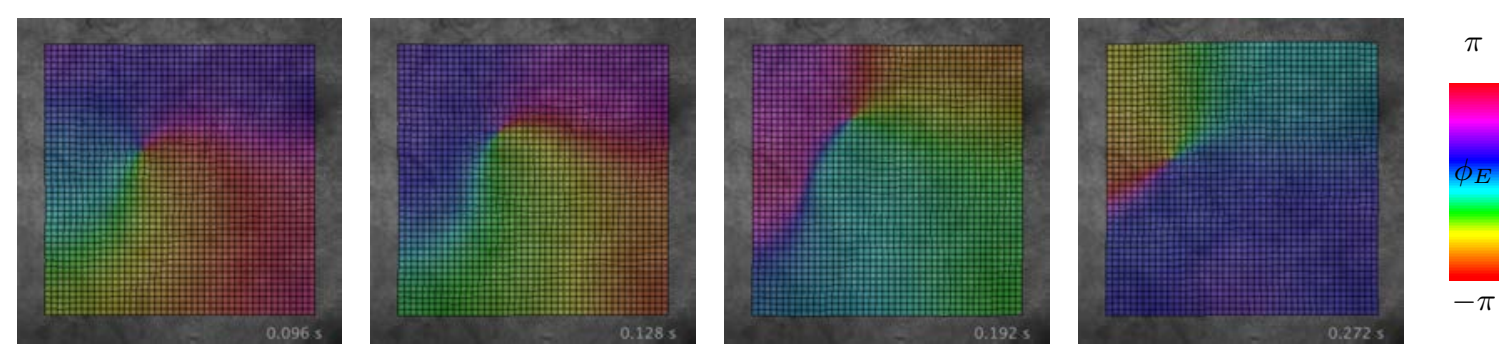

Figure 5.31: Elasto-mechanical spiral wave rotor in engineered cardiac cell culture: phase representation $\phi_{E}$ of elasto-mechanical rotor pattern. Analysis as presented in section 5.3.1 yields phase singularity at rotational center of mechanical rotor. Note the similarity with the simulated spiral wave pattern shown in figure 5.8 .

\subsubsection{Electromechanical Spiral Wave Tracked in Experimental Data of Engineered Cardiac Cell Culture}

The elastic deformation tracking technique was used to track an electromechanical spiral wave imaged inside an engineered cardiac cell culture preparation, see figure 5.30. The cell culture preparations exhibited strong spiraling contractile motion that resulted in large deformations that were clearly macroscopically visible. The preparations were $\sim 6 \mathrm{~mm}$ in diameter and about $2-3 \mathrm{~mm}$ thick and appeared to be translucent, such that the spiraling contractile activity could be filmed using a confocal macroscope, back-illumination and a high-speed camera (Phantom, Vision Research, 200fps, $800 \times 600$ Pixel). Figures 5.30 and 5.31 show the spiraling contractile elasto-mechanical wave activity within the central horizontal plane $(\mathrm{z}=1,5 \mathrm{~mm})$. The motion and deformation was tracked using the elastic deformation tracking techniques described in the previous sections. The elasto-mechanical deformation pattern exhibits counter-clock-wise rotating activity composed of dilating and contracting rates of deformation. The rotational center is located approximately in the center of the field of view and showed to remain stationary throughout the recording. The spiral performed many rotations, of which $5-6$ rotations $(f \approx 3 H z)$ were captured within the video. The same reconstruction technique that was described in the previous sections produced the phase representation of the mechanical activity $\phi_{E}$ and the resulting phase pattern revealed a phase singularity with counter-clock-wise vorticity at the center of the pattern. The recording suggests that the rotational center of the mechanical pattern is co-localized with the core of the underlying action potential spiral wave, that caused the tissue to deform appropriately. Note the good agreement with the simulated spiral wave pattern shown in figure 5.8 in section 5.3.1. 
Chapter 5. Electromechancial Wave Pattern Reconstruction

The engineered cardiac cell culture preparations were provided and also imaged by C. Richter. Neonatal ventricular rat cardiomyocytes were isolated from excised hearts of 1 to 2 day old Wistar rats. Hearts were extracted and processed enzymatically with collagenase II in several digestive steps. Cells were plated on pre-made matrices at an initial density of $1 \times 106$ cells/matrix. To fabricate three-dimensional cell culture matrices, collagen was used to form stable jelly-like scaffolding substrates. Hydrogels were made out of collagen from mouse-tails, phosphate buffer saline

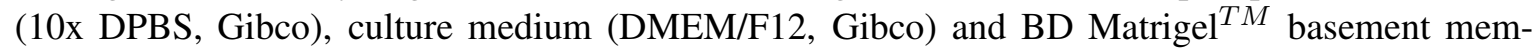
brane matrix (BD Biosciences). The $\mathrm{pH}$ was neutralized by sodium hydroxide. Right before further processing, crosslinking of collagen was initialized by thrombin and fibrinogen (Sigma GmbH, Germany). The solution was rapidly transferred onto printed support structures (using the 3D printer $\mathrm{k} 8200$, Vellemann), which consisted of several honeycomb shaped compartments. The matrices were allowed to fully crosslink under $37^{\circ} \mathrm{C}$ for $5 \mathrm{~min}$ before culture medium was added. Cells were cultivated under physiological conditions and medium was replaced after $24 h, 48 h$ and every following $48 h$. 
Chapter 6. Intramural Scroll Wave Imaging during Ventricular Tachycardia and Fibrillation

\section{Chapter 6}

\section{Intramural Scroll Wave Imaging during Ventricular Tachycardia and Fibrillation}

Scroll waves are conjectured to be the self-organizational, spatial-temporal wave pattern underlying cardiac fibrillation. . $^{40-50,59,65,229}$ To date, however, a panoramic and volumetric visualization of the wave pattern inside the heart muscle evades experimenal realization. ${ }^{200}$ This lack of imaging is considered to be the missing key ${ }^{48,103}$ for the understanding of cardiac tachyarrhythmias such as ventricular tachycardia and fibrillation. Understanding the emergence and formation of the highly dynamic action potential wave pattern which underlies cardiac fibrillation, is necessary to develop strategies for its control. For instance, recent low-energy anti-fibrillation pacing techniques ${ }^{177,203}$ postulate to be able to interact with scroll wave rotor cores using pulsed electric fields and structural heterogeneities ${ }^{227}$ of the underlying anatomic substrate to annihilate fibrillatory activity. However, neither interaction of scroll waves with the underlying cardiac substrate nor the interaction of antifibrillation pacing with scroll wave activity was ever imaged.

In this chapter, it is demonstrated that scroll waves can be imaged in intact, isolated Langendorffperfused rabbit hearts intramurally within the heart muscle during ventricular tachycardia and fibrillation. It is shown that by employing the excitation-contraction coupling mechanism and targeting contractile motion and resulting mechanical deformation of the cardiac muscle, it becomes possible to identify the underlying electrical activation pattern that caused the heart muscle to deform. Both electrical and mechanical activity patterns were filmed using high-speed fluorescence and ultrasound imaging simultaneously. Analysis and comparison of electrical and mechanical spatial-temporal patterns on the surface and inside the heart wall revealed that electrical and mechanical patterns are closely coupled and show correspondingly similar features that allow the identification of organizational centers of rotating waves. More specifically, in section 6.6.1 it is shown that it is possible to associate rotating electrical activity with rotating mechanical activity during fibrillation and that the rotational centers of both of these rotating patterns are co-localized on the epicardial surface. Moreover, section 6.6.2 demonstrates that this close correlation can also be found inside the cardiac muscle can also also when imaging at midwall, rotational mechanical patterns.

This chapter begins with a presentation of the experimental design and a formulation of the hypothesis. Sections 6.1 through 6.5 present the experimental and computational methods which were developed and used for the simultaneous imaging and analysis of electrical and mechanical activity in intact hearts in vitro. Section 6.6 demonstrates the results. 

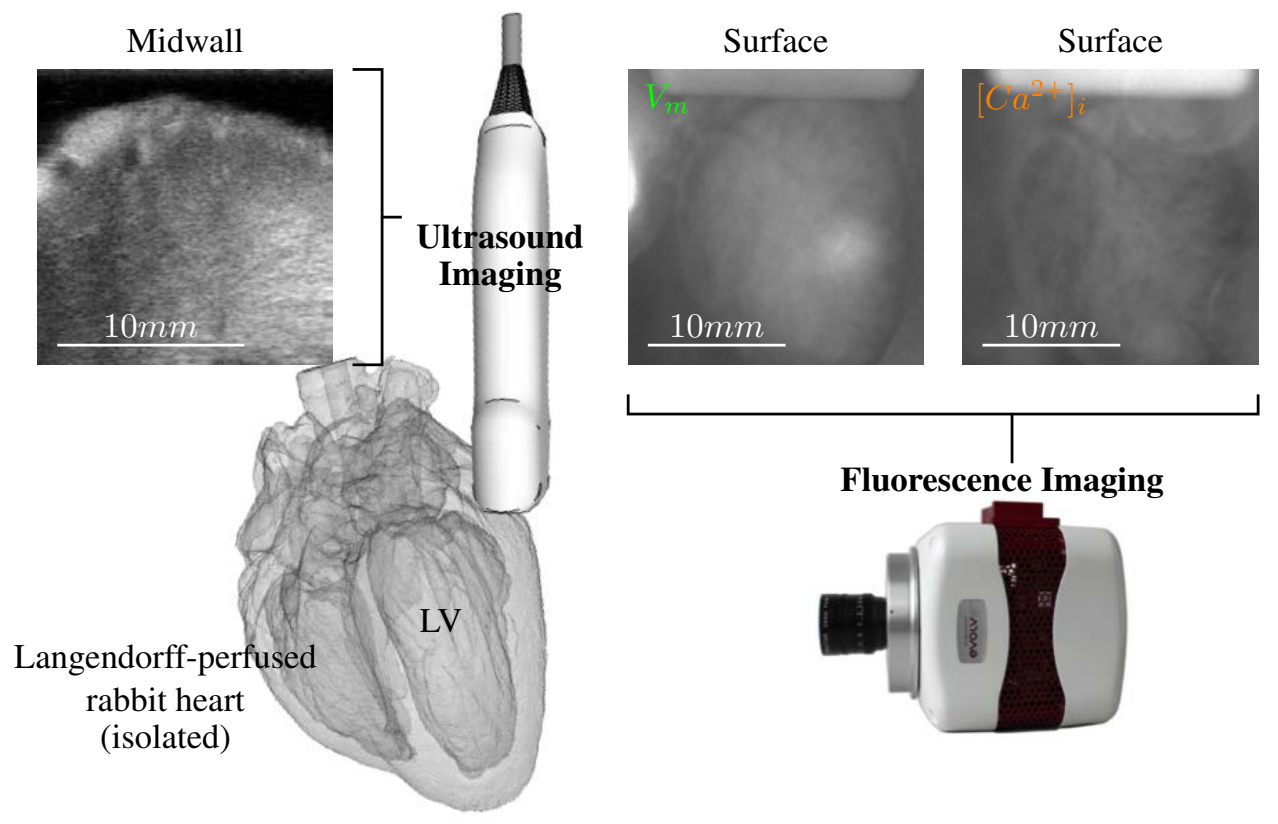

Figure 6.1: Experimental design for simultaneous measurement of electrical and mechanical activity in the heart: intact Langendorff-perfused rabbit heart imaged with optical fluorescence and ultrasound imaging simultaneously to capture coupled electromechanical activity during cardiac arrhythmias on the surface as well as inside the cardiac muscle.

\subsection{Experimental Design and Imaging Setup}

To image both electrical and mechanical activity in the heart, high-speed fluorescence imaging was used in combination with high-speed ultrasound imaging. Both measurement techniques were used simultaneously with their clocks synchronized to acquire movies of the electrical as well as according mechanical activity of the cardiac muscle, at the same time on its surface as well as within cross-sections inside the muscle. The experiments were conducted with isolated rabbit hearts, which were kept in retrograde Langendorff-perfusion, see figures 6.1-6.4. This allowed to conduct experiments with intact hearts ex-vivo under physiological conditions, being able to film coupled electromechanical wave activity during normal sinus rhythm as well as during induced and controlled cardiac tachyarrhythmias, such as ventricular tachycardia and fibrillation.

Figure 6.1 shows the principle design of the experiment. The setup allows to image (a) the fluorescence on the surface, (b) the deformation of the surface and (c) the deformation several millimeters underneath the surface, within the cardiac muscle, at high speeds. The two images on the right show the left ventricular surface of the heart as imaged with fluorescence imaging, with the left image showing voltage-sensitive $\left(V_{m}\right)$ fluorescence emission and the right image showing calcium-sensitive $\left(\left[\mathrm{Ca}^{2+}\right]_{i}\right)$ fluorescence emission, see section 6.4. The image on the left shows a cross-section of the left ventricular wall as imaged with ultrasound. The images are still frames from high-speed videos acquired at acquisition speeds ranging in between $250-500$ frames per second to image the fast electrophysiological processes and mechanical contractions appropriately. All three videos show the contracting and deforming heart and therefore can be used to track and capture the time-varying 


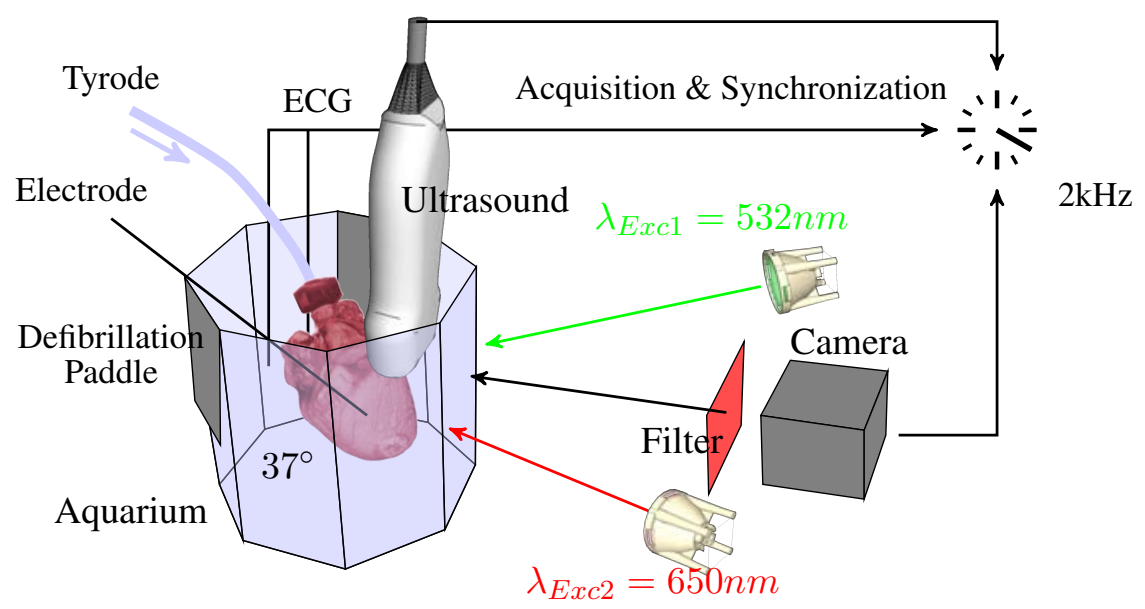

Figure 6.2: Schematic drawing of experimental setup for electromechanical wave imaging in intact, isolated rabbit hearts. Rabbit heart inside perfusion bath connected to retrograde Langendorff-perfusion. Synchronized acquisition of fluorescence and ultrasound videos and electrocardiogram. Simultaneous high-speed fluorescence and ultrasound imaging in left ventricular wall to measure electrophysiological activity and mechanical deformation.

elasto-mechanical activity. However, in addition to the elasto-mechanical activity, the two videos on the right contain fluorescence, which carries information about the electrophysiological activity. In total, the imaging configuration allows to acquire five spatial-temporal patterns, three related to mechanical activity and two related to electrical activity.

Figure 6.2 shows a schematic drawing of the setup and figures 6.3 and 6.5 show the corresponding photographs. The heart is placed inside an eight-sided aquarium filled with heated Tyrode solution at $37^{\circ} \mathrm{C}$ and connected to retrograde Langendorff-perfusion, see section 6.3. The tubing that connects the perfusion to the aorta holds the heart in place. The camera capturing the fluorescence emission films the heart's ventricular surface through one of the glas windows of the aquarium. The ultrasound transducer head is immersed from the top into the bath, filming the ventricular wall from the top directed towards the apex, see figure 6.4.

The idea of the imaging configuration was to image the same part of the heart using both imaging modalities and to align the two imaged areas in a way that the captured two-dimensional patterns of the respective electrical and mechanical activities would show two congruent areas. Therefore, the ultrasound imaging plane was positioned and aligned within the ventricular wall with its plane normal facing the camera. Accordingly, the in-plane horizontal orientation was aligned along the circumferential direction of the heart, with the plane being located approximately at midwall in parallel with the epi- and endocardial walls, see figures 6.3, 6.4 and 6.18. It was assumed that the muscle fiber orientation would also lie within or approximately parallel to the imaging cross-section. Accordingly, the camera filming the surface showed a projection of the surface that was parallel to the internal cross-section.

Next to filming, the setup also allowed to record electrocardiograms and to induce and terminate 

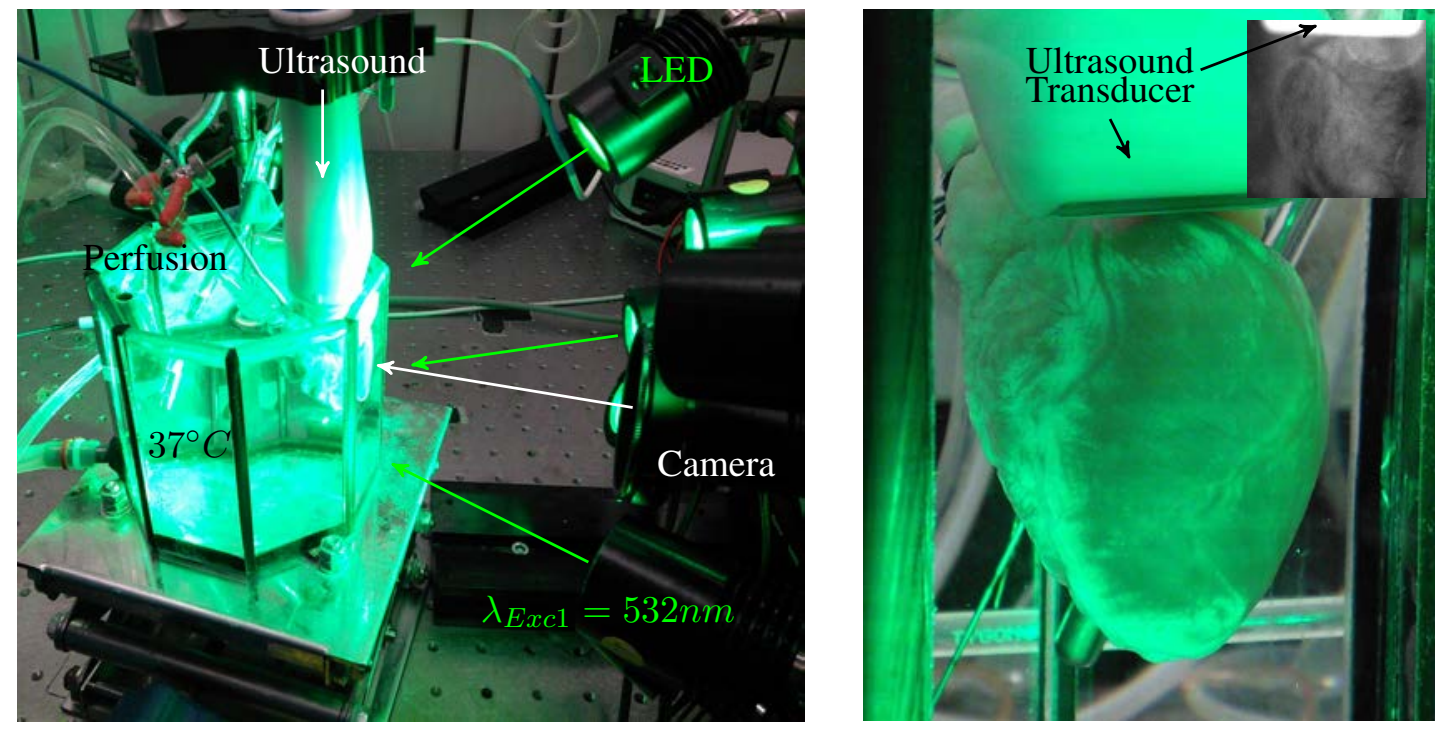

Figure 6.3: Experimental setup with simultaneous optical fluorescence and ultrasound imaging: (a) Imaging configuration with scanning position of ultrasound transducer for cross-sectional imaging of left ventricular wall: (a) perfusion tubing holding heart in position with left ventricular wall facing glass wall of aquarium (b) ultrasound transducer intersecting lect ventricular wall from the top imaging cross-section of wall at midwall as indicated in figure 6.5, epicardial surface visible to camera (c) camera and diode positioning in front of bath, camera filming left ventricular surface
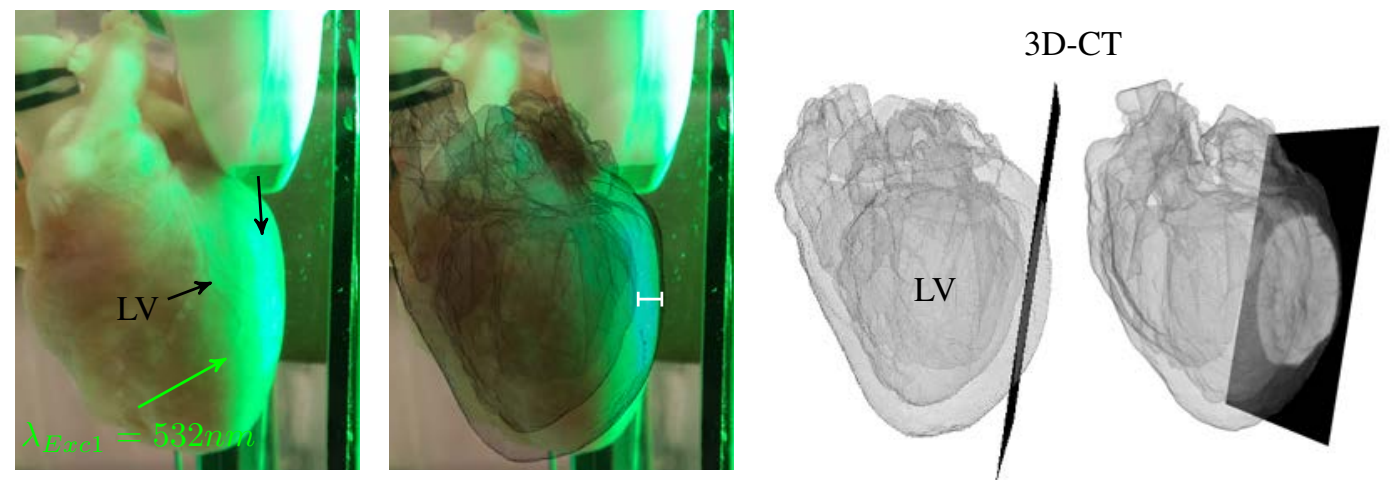

Figure 6.4: Imaging configuration with scanning position of ultrasound: side view of ultrasound transducer scanning head and rabbit heart during imaging with ultrasound imaging plane intersecting left ventricular wall with the plane located approximately at midwall in parallel to epi- and endocardial walls to image a circumferential cross-section. Wall thickness of rabbit heart about $2-5 \mathrm{~mm}$. Illumination from the right at $\lambda_{E x c 1}=532 \mathrm{~nm}$. 
Chapter 6. Intramural Scroll Wave Imaging during Ventricular Tachycardia and Fibrillation

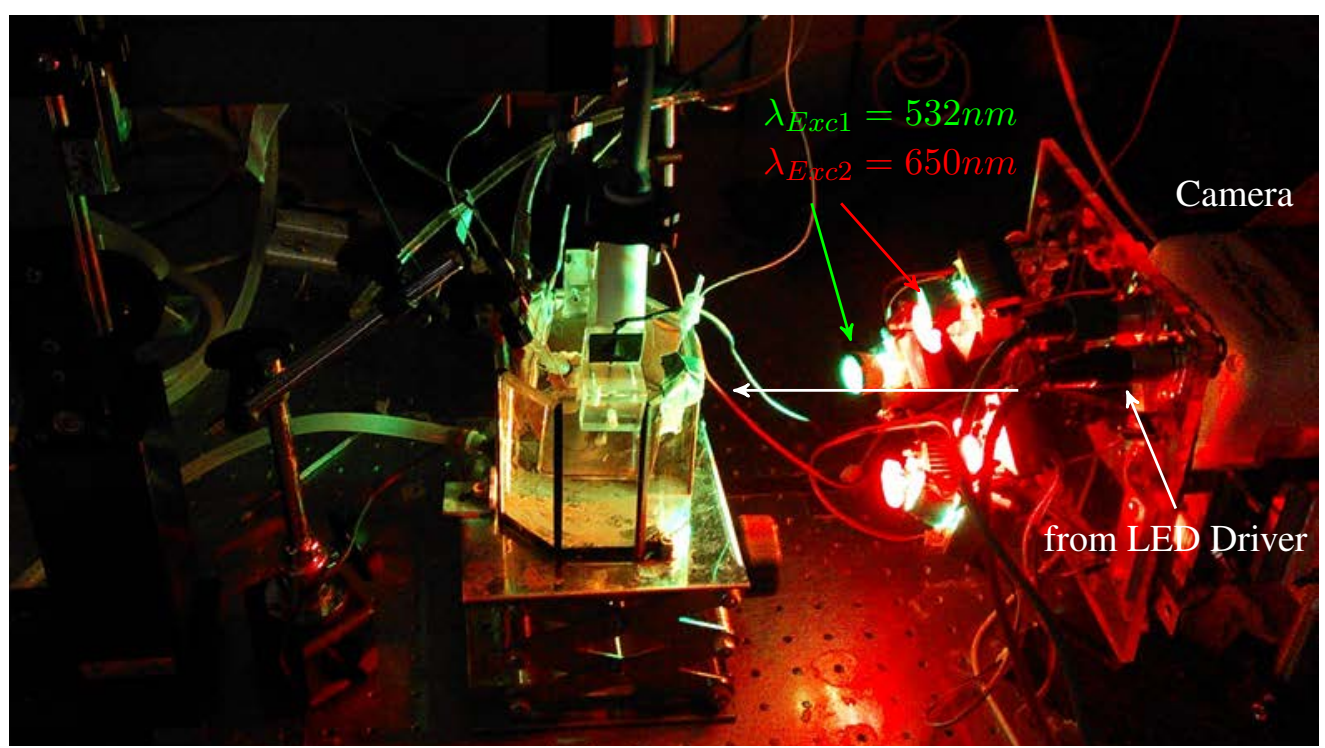

(a)

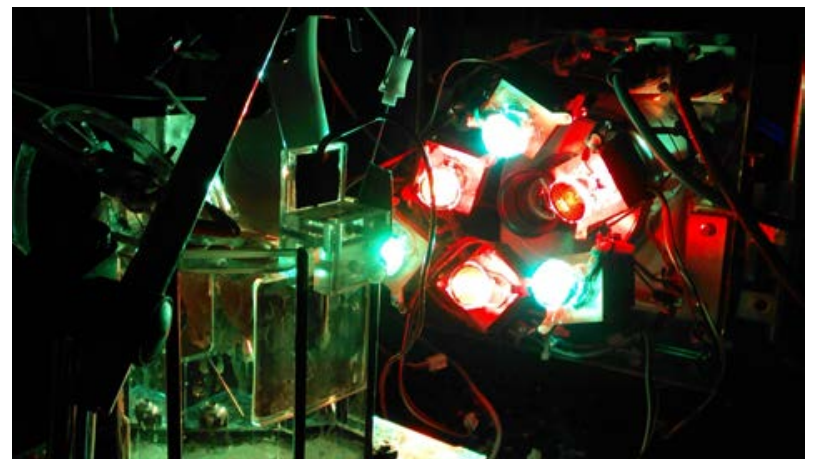

(b)

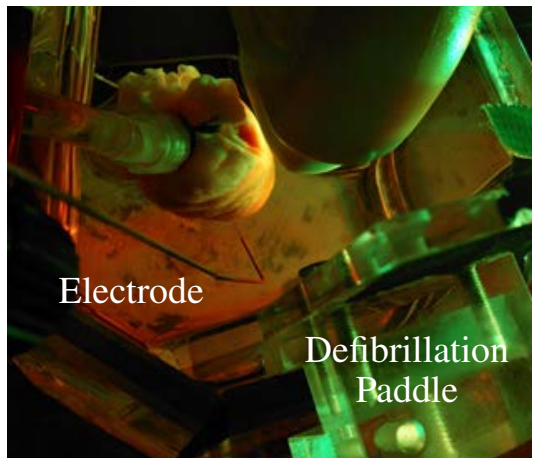

(c)

Figure 6.5: Experimental setup with multi-parametric fluorescence imaging to measure transmembrane voltage $V_{m}$ and intracellular calcium concentration $[\mathrm{Ca}]^{2+}$ on the epicardial surface as well as mechanical deformation on surface, as well as intramural mechanical deformation inside the ventricular wall using ultrasound imaging. Two excitation bandwidths at $\lambda_{E x c 1}=532 \mathrm{~nm}$ to excite calcium-sensitive dye and $\lambda_{E x c 2}=650 \mathrm{~nm}$ to excite voltage-sensitive dye and rapid switching of illumination at $500 \mathrm{fps}$ ( $250 \mathrm{fps}$ per parameter, see below).

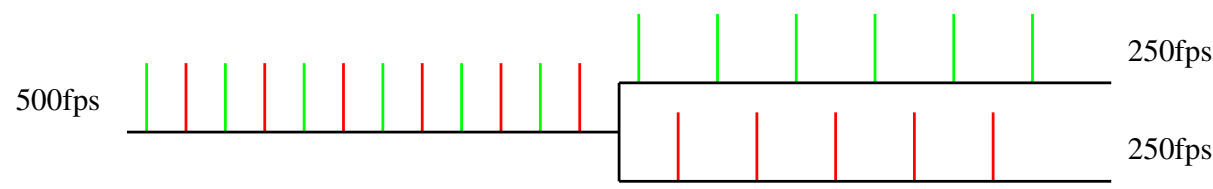

Figure 6.6: Switching scheme of LED illumination for multi-parametric imaging: custom-made driver switching illumination every $2 \mathrm{~ms}$ from red to green light for interleaved excitation of two dyes for multi-parametric imaging, see section 6.4, resulting in an effective acquisition speed of $250 \mathrm{fps}$ per acquired parameter. 
arrhythmias, see section, by applying pacing pulses, cardioversion shocks or low energy antifibrillation pacing (LEAP). To be able to asociate the according frames of the fluorescence and ultrasound recordings with each other and with the electrocardiogram recordings, a commercial data acquisition was used, see section, recording the trigger signals of each device. Recordings were acquired and analyzed using custom-made acquisition and post-processing software, see following sections, to visualize spatial-temporal patterns of electrical and mechanical activity respectively. Experiments were conducted together with M. Chebbok. In total, 20 experiments were conducted in between June 2012 and July 2014.

\subsection{Electromechanical Coupling Hypothesis}

In this work, it is assumed that when imaging cardiac fibrillatory activity the excitation-contraction coupling mechanism manifests in two closely correlated electrical and elasto-mechanical patterns, see also chapter 5. This requires that the excitation-contraction coupling does not degenerate as it may be the case, for instance, during so-called early-after depolarizations, during which calcium cycling may trigger transmembrane potential depolarizations. Here, it was assumed that electromechanical coupling would lead to homogenous active tension development and conversion of excitation into myofibril contraction. At the same time, it was expected that the anisotropic cardiac substrate, including its vasculature and muscle fiber anisotropy, would, nevertheless, allow to observe two closely related electrical and mechanical activity patterns.

\subsection{Tissue Preparation and Arrhythmia Control}

New Zealand white rabbits were anaesthesized using $4.0 \mathrm{ml}$ Trapanal (Thiopental-sodium solution) diluted in $10.0 \mathrm{ml}$ isotonic sodium chloride $(\mathrm{NaCl})$ solution and hearts were excised rapidly. Tyrode solution ( $15 \mathrm{l}$ distilled water, $130 \mathrm{mMol}$ or $114 \mathrm{~g}$ sodium-chloride $(\mathrm{NaCl}), 4 \mathrm{mMol}$ or $4.47 \mathrm{~g}$ potassium-chloride $(\mathrm{KCl}), 0.6 \mathrm{~m} \mathrm{Mol}$ or $1.83 \mathrm{~g}$ magnesium-chloride hexahydrate $\left(\mathrm{MgCl}_{2}\left(6 \mathrm{H}_{2} \mathrm{O}\right)\right.$ ), $2.2 \mathrm{mMol}$ or $3.63 \mathrm{~g}$ calcium-chloride $\left(\mathrm{CaCl}_{2}\right), 1.2 \mathrm{mMol}$ or $2.16 \mathrm{~g}$ sodiumdihydrogen-phosphate $\mathrm{NaH}_{2} \mathrm{PO}_{4}\left(\mathrm{H}_{2} \mathrm{O}\right), 24.2 \mathrm{mMol}$ or $30.45 \mathrm{~g}$ sodium-hydrogen-carbonate $\left(\mathrm{NaHCO}_{3}\right)$ and $12 \mathrm{mMol}$ or $32.4 g$ glucose, ph-level 7.36-7.40) was prepared as described. Isolated hearts were transferred quickly into a bath filled with warm Tyrode and connected to a retrograde Langendorff-perfusion setup as described. Tyrode was kept at a temperature of $T=37.0^{\circ} \mathrm{C} \pm 0.5^{\circ} \mathrm{C}$ (custom-made temperature control, Max Planck Institute for Dynamics and Self-Organization, Göttingen, Germany) and was re-perfused. Uncoupling agents such as Blebbistatin ${ }^{102}$ (Sigma-Aldrich, St. Louis, USA) or DAM (diacetyl monoxime) were not applied.

Arrhythmias were induced and terminated using pacing electrodes and far-field defibrillation paddles. Electrocardiograms were recorded using two thin electrodes, see figure 6.2, inserted into the bath and acquired using a data acquisition system (MP150, Biopac Systems, Inc., Goleta, USA) and according acquisition software (AcqKnowledge, Biopac Systems, Inc., Goleta, USA) acquiring data at a sampling rate of $2 \mathrm{kHz}$ throughout the entire duration of the experiment. The same system allowed to control and receive sensing signals from an integrated electrode designed to capture the monophasic action potential (MAP-electrode). The MAP-electrode was positioned inside the right ventricle, touching the endocardial wall with its tip. Both signals from the MAP-electrode and the electrocardiogram were used to identify activation patterns and distinguish between sinus rhythm and 
ventricular tachycardia and fibrillation. To induce ventricular tachyarrhythmias, rapid pacing stimuli were applied using the MAP-electrode or an externally positioned bipolar electrode that touched the epicard of the left ventricular wall, see figure 6.5(c). The external electrode was powered $(20 \mathrm{~mA})$ by a stimulus isolator box (Stimulus Isolator, A385, World Precision Instruments, Sarasota, USA). Both electrodes could be controlled by custom-made software (LabView, Biomedical Physics Group, Max Planck Institute for Dynamics and Self-Organization, Göttingen, Germany). Pacing for tachyarrhythmia induction consisted of a sequence of bi-phasic pulses (1.0 $\mathrm{V}$ amplitude, $5 \mathrm{~ms}$ duration) for an overall duration of $1.0 \mathrm{~s}$ to $5.0 \mathrm{~s}$ seconds at frequencies of $f_{1}=20 \mathrm{~Hz}$ or $f_{2}=50 \mathrm{~Hz}$ respectively. Output of both electrodes was recorded using the data acquisition system. Arrhythmias were terminated using low-energy anti-fibrillation pacing ${ }^{203}$ (LEAP) protocols ( 5 pulses, $8 \mathrm{~ms}$ duration, $70 \mathrm{~ms}$ to $100 \mathrm{~ms}$ cycle length, $70.0 \mathrm{~V}$ ) or cardioversion shocks $(70.0 \mathrm{~V})$. Shocks were delivered through 2 plate electrodes (paddles) inserted into the bath, see figure 6.2 and figures $6.5(\mathrm{~b}-\mathrm{c})$. The electrical power was supplied by a power amplifier (Kepco Power Supply, Flushing, USA). Also, the power amplifier output was recorded using the data acquisition system.

\subsection{Fluorescence Imaging}

Fluorescence imaging was performed using a monocular single-camera setup. The imaging setup allowed to measure either a single parameter, the transmembrane voltage $V_{m}$, or two parameters simultaneously, the transmembrane voltage $V_{m}$ as well as the intracellular calcium concentration $\left[\mathrm{Ca}^{2+}\right]_{i}$ in a special multi-parametric imaging mode. ${ }^{202,217,218}$ However, only a small number of experiments were performed in the multi-parametric imaging mode. Due to the complexity of the experiments, panoramic imaging with multiple cameras was not performed.

The following section describes the methods built or developed for the task to image the electrophysiological activity, that are action potential and calcium transient wave patterns, in intact and contracting rabbit hearts with emphasis on handling of the contractile motion, see section 6.4.3, which was a particular challenge during the fluorescence imaging experiments.

\subsubsection{Optical Imaging Setup and Data Acquisition}

Optical mapping recordings were performed using high-speed cameras (Photometrics Cascade / Evolve, $500 \mathrm{fps}, 128 \times 128$ pixels) together with a fast lens and objective $(52 \mathrm{~mm}$, f 0.95-16, $0.2 \mathrm{~m}-$ $10.0 \mathrm{~m}$, Tokina, Japan) allowing to film fields of view of about $1.5 \mathrm{~cm} \times 1.5 \mathrm{~cm}$ to $2.0 \mathrm{~cm} \times 2.0 \mathrm{~cm}$. In mono-parametric imaging mode, green excitation light was provided by 12 high performance light emitting diodes ( $\lambda=532 \mathrm{~nm} \pm 10 \mathrm{~nm}$ wavelength). In multi-parametric imaging mode, green and red excitation light was provided by 6 high performance light emitting diodes $(P=5.0 \mathrm{~W}$, broad bandwidth, Hersteller). Light of 3 diodes was filtered using green bandpass filters $\left(\lambda_{E x c 1}=\right.$ $550 \mathrm{~nm} \pm 5 \mathrm{~nm}$, Hersteller) and light from 3 diodes was filtered using red bandpass filters $\left(\lambda_{E x c 2}=\right.$ $640 \mathrm{~nm} \pm 5 \mathrm{~nm}$, Hersteller). To extract emission light, overall light was filtered using red/nearinfrared longpass or bandpass filters respectively before passing through the objective and being captured by the camera sensor. In mono-parametric imaging mode, a longpass filter was used to collect emission light with wavelengths $\lambda>650 \mathrm{~nm}$. In multi-parametric imaging mode, a dualbandpass filter was used to collect light from the two emission bandwidths with wavelengths ranging from $\lambda_{E m 1}=560 \mathrm{~nm}$ to $610 \mathrm{~nm}$ and $\lambda_{E m 2}>700 \mathrm{~nm}$ respectively. ${ }^{217}$ Cameras were cooled at $25^{\circ} \mathrm{C}$ using a liquid cooling system (Exos Cooling System, Koolance, Auburn, USA). Design principles of 
optical mapping systems are described in section 3.1.

In multi-parametric imaging mode, ${ }^{202,217,218}$ the two channels, transmembrane voltage $V_{m}$ and intracellular calcium concentration $\left[\mathrm{Ca}^{2+}\right]_{i}$ were captured in every other frame, recording an interleaved sequence of frames with the even frames showing the voltage channel and the odd frames showing the calcium channel. Accordingly, the power supply of the light emitting diodes was switched from one channel ( $550 \mathrm{~nm}$ excitation light) to the other (650 $\mathrm{nm}$ excitation light) from frame to frame throughout the sequence using a custom-made diode driver box (Flavio Fenton and Ilja Uzelac, School of Physics, Georgia Institute of Technology, Atlanta, USA). Correspondingly, in multi-parametric imaging mode the acquisition speed was $250 \mathrm{fps}$ per channel, whereas in mono-parametric imaging mode the acquisition speed was $500 \mathrm{fps}$.

Movies were acquired using custom-made recording software (MultiRecorder, version 2.0.9, Biomedical Physics Group, Max Planck Institute for Dynamics and Self-Organization, Göttingen, Germany). Camera trigger signals were provided by a function generator $5.0 \mathrm{~V}$ TTL-signal at repetition rates of $500 \mathrm{~Hz}$ resulting in the acquisition speed or frame rate of the cameras, 20MHz Function / Waveform Generator, model 33220A, Agilent, Santa Clara, USA). Camera trigger signals were captured using a data acquisition system (MP150, Biopac Systems, Inc., Goleta, USA) and according acquisition software (AcqKnowledge, Biopac Systems, Inc., Goleta, USA). Start and end of the recordings could be associated with the start and end times of the camera trigger signals.

Hearts were filmed from the side through the glass-walls of the eight-sided aquarium at a distance of about $d=20.0 \mathrm{~cm}$ as depicted in figure 6.5. Other imaging configurations showed to be problematic. Filming from the top, for instance, created the problem that undesired motion of the water surface created strong optical abberations. This problem could be circumvented by covering the water surface with a plexi-glas plate. However, the plexi-glass plate complicated handling of the heart and eventually caused pressure onto the heart. At the same time, it showed to be more practical to scan from the top with the ultrasound transducer. Different attempts to inhibit translational motion of the heart mechanically using molds or other devices for fixation prevented proper perfusion of the heart. Presumably, mechanical pressure blocked the coronary vasculature.

\subsubsection{Staining and Image Properties of Fluorescence Video Data}

Tissue preparations were stained with fluorescent dye to visualize the fractional change $\Delta F / F$ of fluorescence emission intensity $F$ that is directly related to the electrophysiological activity. For instance, the depolarization of the transmembrane potential $V_{m}$ of each cell causes a slight shift of the emission spectrum of attached voltage-sensitive fluorophores and the use of filtering techniques that collect and cut the emission spectrum appropriately produces a decrease of captured fluorescent light on the camera detector. This leads to a signal that typically shows the inverted shape of an action potential, see also section 3.1. Dye was loaded via retrograde Langendorff-perfusion ${ }^{197}$ through the heart's vasculature and capillary system into the myocardial muscle, see also chapter 3.1. In mono-parametric imaging mode, tissue preparations were stained with voltage-sensitive / potentiometric fluorescent dye (Di-4-ANEPPS, $20 \mathrm{ml}$ of $1 \mu \mathrm{Mol}$ concentrated dye-Tyrode solution) only. In multi-parametric imaging mode, tissue preparations were stained with voltage-sensitive / potentiometric dye (Di-4-ANBDQPQ, $20 \mathrm{ml}$ of $2.5 \mu \mathrm{Mol}$ concentrated dye-Tyrode solution) as well as calcium-sensitive fluorescent dye (AM-Rhod2, $20 \mathrm{ml}$ of $2.5 \mu \mathrm{Mol}$ concentrated dye-Tyrode solution). ${ }^{217}$ Typically, half of the dye bolus was injected directly into the perfusion path, the other half 


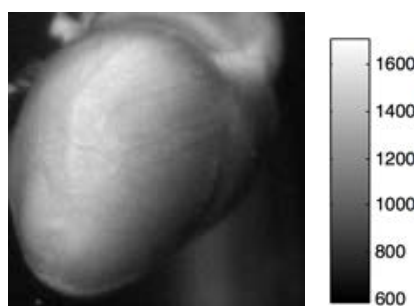

$0.0 \mathrm{~s}$

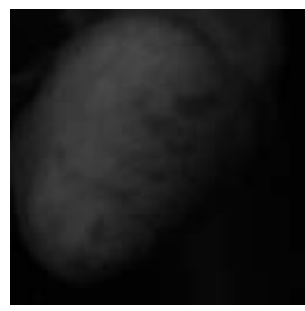

$9.0 \mathrm{~s}$

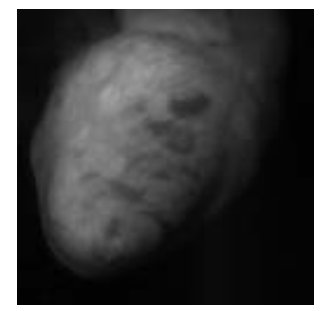

$20.0 s$

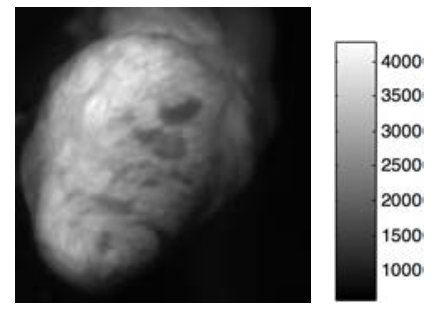

$30.0 \mathrm{~s}$

Figure 6.7: Effect of staining on image properties of fluorescence video data: left image showing rabbit heart without staining, image normalized to display values from 1-1000 counts to facilitate viewing, following images normalized to minimum and maximum values of entire sequence (1-4000) to facilitate viewing of the increase of fluorescence emission intensity $F$ during staining after $9.0 \mathrm{~s}$, $20.0 s$ and $30.0 s$.

was injected into the bubble trap. Recordings were typically started within few minutes after the injection of the dye. Washed-out dye was constantly re-perfused.

\section{Fluorescence Emission Intensity and Fractional Change $\Delta F / F$}

Figure 6.7 shows the effect of staining onto the image properties of optical fluorescence recording data. The left image in the sequence shows a Langendorff-perfused rabbit heart as imaged through the emission filter before staining. To facilitate viewing, the image was normalized to display 1-1000 counts detected by the 16-bit camera sensor accordingly. The following images to the right show the same rabbit heart at $9.0 s, 20.0 s$ and $30.0 s$ respectively after the injection of dye Di-4-ANEPPS. Here, the images are normalized to display 1-4000 detected pixel counts to facilitate viewing of the increase of the emission intensity $F$ during this period. It is easy to note that the appearance of the heart changes significantly. Some larger dark spots on the epicardial surface of the heart indicate uneven dye loading, that is dye is perfusing heterogeneously through the different parts of the muscle. This effect can be observed to become less dramatic and to disappear over the following minutes as dye is constantly re-perfused and eventually perfused more evenly, compare also with figures 6.9 and 6.10 .

Figure 6.8 shows the correspondingly measured increase in fluorescence emission intensity over the first $30.0 \mathrm{~s}$ of the staining procedure. The three black curves in the top left panel show (from top to bottom) the increase of the maximum intensity value or peak emission intensity $F_{\text {peak }}(t)$, the mean fluorescence emission intensity $F_{\text {mean }}(t)$ and the minimal fluorescence emission intensity $F_{\min }(t)$ measured in counts $I$ detected by the camera sensor. Both mean and minimal curves were measured from pixels $(x, y)$ lying inside an interrogation area $\mathcal{A}$, enclosed by the red line in the video frame, which was found to show tissue throughout the entire sequence. The peak emission intensity $F_{\text {peak }}(t)$ was computed as follows from the video data:

$$
F_{\text {peak }}(t)=\max I(x, y, t)
$$

where $I(x, y, t)$ is the respective captured video frame at time $t$ with $I$ intensity counts in each pixel $(x, y)$ and all pixels of the entire frame were considered. Intensity counts ranged in between 0 and $2^{16}-1=65,536$ possible values or counts corresponding to the 16-bit dynamic range of the camera. Pixels $(x, y)$ are arranged on a two-dimensional pixel detector array with dimensions $N \times M$ and 

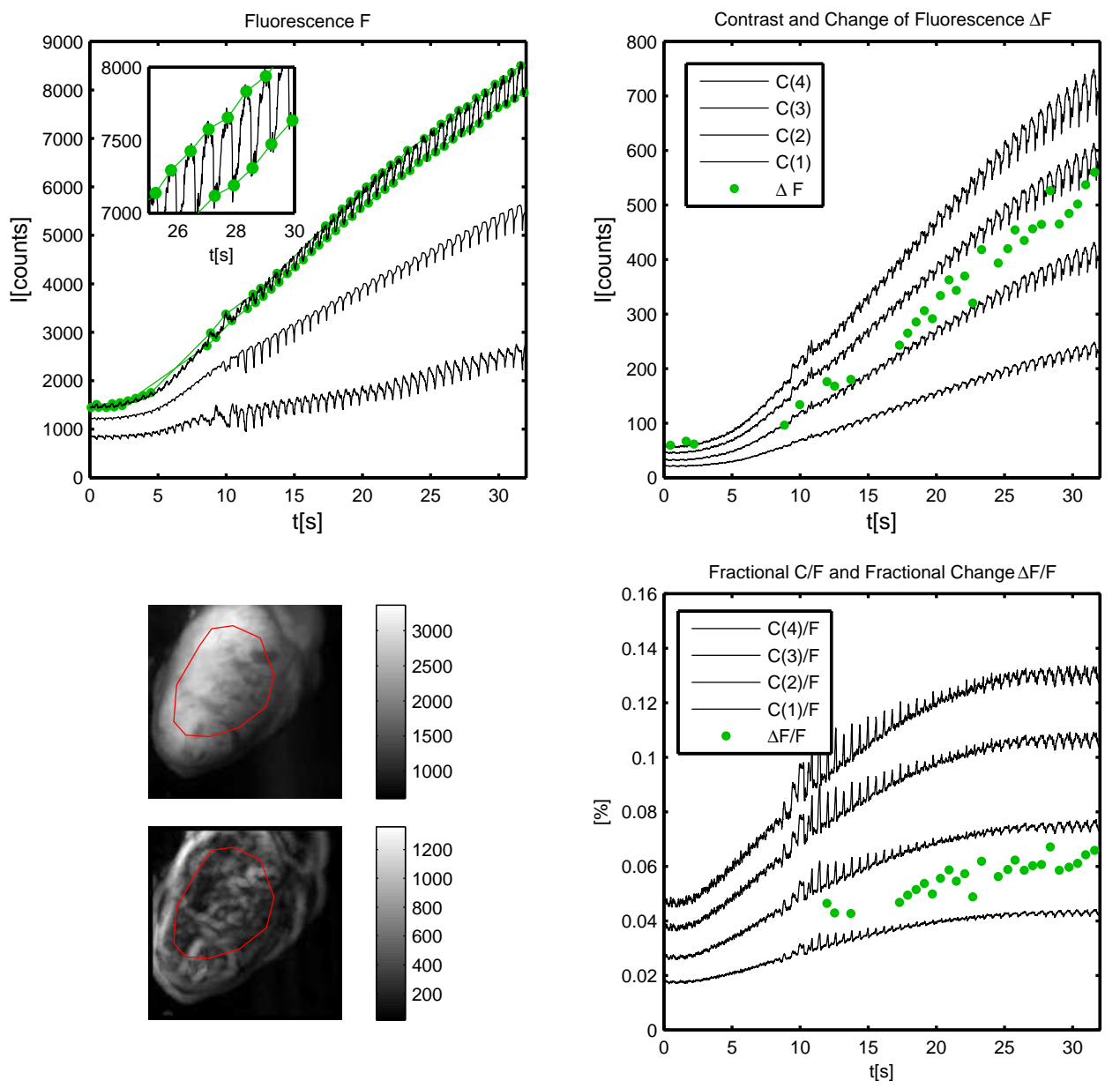

Figure 6.8: Fluorescence and image contrast during loading of dye in rabbit heart. Top left panel: increase in fluorescence emission intensity $F$ measured in absolute counts (upper curve: peak intensity $F_{\text {peak }}$, middle curve: mean intensity $F_{\text {mean }}$, lower curve: minimal intensity $F_{\text {min }}$ derived from red region indicated in video frame) in first $30.0 \mathrm{~s}$ after beginning of staining procedure with Di-4ANEPPS fluorescent dye. Fluctuations of fluorescence intensity due to electrical (and mechanical) activity at frequency of heart rate. Peak and minima detection for estimation of fractional change $\Delta F / F$ of peak fluorescence intensity, assumed that peak intensity independent of motion. Top right panel: increase in local image contrast $C$ related to fluorescence emission heterogeneity and tissue structure. Computed for a circular-shaped subregion with varying radii $r=1,2,3,4$ pixels. Fractional change of fluorescence emission intensity $\Delta F$ in counts (green dots) as derived from maxima and minima detection of peak intensity $F_{\text {peak }}$. Image contrast stays larger than intensity variations caused by electrophysiological activity. Bottom left panel: video still image $10.0 \mathrm{~s}$ after start of the staining procedure and contrast image with area of interest $\mathcal{A}$. Bottom right panel: fractional contrast $C / F$ for different subregion sizes with radii $r=1,2,3,4$ pixels and fractional change $\Delta F / F$ of fluorescence intensity. Compare also with figure 6.13. 
$N=M=128$ pixels respectively. The minimal fluorescence emission intensity $F_{\min }(t)$ was computed analogously as in equation 6.4.1, however, extracting the minimal grayvalue in each frame with pixels $(x, y) \in \mathcal{A}$. In this sequence, it was assumed that the brightest pixel in the frame was an adequate measure for the amount of loaded dye that also reflects the fractional change $\Delta F / F$ due to electrical activity sufficiently well. The brightest pixel was assumed to be a specific site on the moving substrate that would be detected despite motion as the maximal count in the frame. Indeed, $F_{\text {peak }}(t)$ exhibits periodic intensity fluctuations and the typical steep downstrokes, see also subgraph in the same panel, which are caused by the rapidly depolarizing membrane and change in transmembrane potential $V_{m}$, see also section 3.1. The mean fluorescence emission intensity $F_{\text {mean }}(t)$ per frame was computed as follows:

$$
F_{\text {mean }}(t)=\frac{1}{N \cdot M} \sum_{x}^{N} \sum_{y}^{M} I(x, y, t)
$$

with $(x, y) \in \mathcal{A}$. All three curves show a strong increase during the first $30.0 \mathrm{~s}$ of the staining procedure. The peak intensity $F_{\text {peak }}(t)$ exhibits a more than five-fold increase in intensity compared to the baseline without staining. All three curves start exhibiting periodic intensity fluctuations $\Delta F$ only a few seconds after the beginning of the staining procedure. However, while certainly in all three traces the electrophysiological activity causes these fluctuations, here it was assumed that only the peak fluorescence intensity $F_{\text {peak }}(t)$ contained a more or less motion-artifact free change $\Delta F$ that could be associated solely with electrical activity. The other two curves were considered to be affected by motion-induced optical flow, see next section. The peak intensity $F_{\text {peak }}(t)$ was used to estimate the magnitude of the change of the fluorescence intensity $\Delta F$ caused by the transmembrane potential $V_{m}$. The change of fluorescence $\Delta F$ was estimated to be the magnitude of the difference of magnitudes $F\left(t_{i}\right)$ and $F\left(t_{j}\right)$ of two subsequent local maxima and minima:

$$
\Delta F\left(t_{k}\right)=\left|F_{\max }\left(t_{i}\right)-F_{\min }\left(t_{j}\right)\right|
$$

with $t_{j}-t_{i}>0$ and $\min _{i, j}\left|t_{i}-t_{j}\right|$ and where the indices $i=1,2,3, \ldots, j=1,2,3, \ldots$ and $k=1,2,3, \ldots$ run over the two sets of detected maxima or minima and detected pairs of maxima and minima respectively. The top right panel in figure 6.8 shows that the magnitude of change of fluorescence $\Delta F$ continously increases (depicted by green dots) and becomes as large as 500 intensity counts and still shows to be increasing at the end of the sequence. The bottom right panel shows the according fractional change of fluorescence $\Delta F / F$, which is a normalized measure of the change of fluorescence compared to the overall fluorescence intensity, and was computed in this case by dividing the change of fluorescence by the baseline of the signal $F_{\text {peak }}(t)$ or the corresponding detected maxima $F_{\max }\left(t_{i}\right)$ respectively. The observed fractional change of $4-6 \%$ is typical for cardiac fluorescence imaging experiments with dye Di-4-ANEPPS.

\section{Image and Tissue Contrast}

In addition to the intensity of the fluorescence, the behavior of the image contrast $C(x, y, t, w)$ was studied. The image contrast reflects the local contrast of intensity grayvalues of the fluorescence emission exhibited by each region of the tissue due to structural heterogeneities such as capillary vessels visible on the epicardial surface or uneven loading or emission of dye. The local image contrast $C(x, y, t, w)$ was computed for every pixel in every frame by finding the largest deviating grayvalue inside a circular-shaped subregion $\mathcal{S}_{(x, y)}$ of the image from the corresponding grayvalue 
of the central pixel $(x, y)$ of the subregion:

$$
C(x, y, t, w)=\max _{x^{\prime}, y^{\prime} \in \mathcal{S}_{(x, y)}}\left|I\left(x^{\prime}, y^{\prime}, t\right)-I(x, y, t)\right|
$$

where $w$ corresponds to the radius of the circular-shaped subregion $\mathcal{S}_{(x, y)}$ around pixel $(x, y)$ with $w=0$ yielding only pixel $(x, y)$ and all pixels $(x, y) \in \mathcal{A}$. In particular, the contrast $C$ is a measure for the spatial gradient of the image's grayvalues and depends on the size of the radius $w$. It does not depend on tissue motion as it is computed for every frame and pixel individually. To obtain a measure for the evolution of the image contrast over time during staining, the frame-wise mean was computed as follows:

$$
C(t, w)=\frac{1}{N \cdot M} \sum_{x}^{N} \sum_{y}^{M} C(x, y, t, w)
$$

Figure 6.8 shows the according evolution of the image contrast during the staining procedure. The top right panel shows the four different curves $C(1, t), C(2, t), C(3, t)$ and $C(4, t)$ of local image contrasts measured in absolute pixel counts with different sizes $w$ of the subregion $\mathcal{S}_{(x, y)}$ and the bottom right panel shows the four corresponding normalized curves, the fractional local image contrasts $C(1, t) / F, C(2, t) / F, C(3, t) / F$ and $C(4, t) / F$ accordingly. Here, the contrast was normalized using the mean fluorescence intensity $F_{\text {mean }}(t)$. All four curves showing the image contrast are continously increasing during the staining procedure and exhibit magnitudes in the same order as the change of fluorescence $\Delta F$ with contrasts becoming larger with increasing subregion size $w$ and, in particular, becoming larger than $\Delta F$ with a radius of the subregion size larger $w>2$ pixels. Also, the contrasts exhibit periodic fluctuations, similarly as the curves showing the fluorescence $F$. Note that the fractional contrast saturates indicating that eventually the rates at which both fluorescence $F$ and contrast $C$ increase become equally large.

Figure 6.9 shows data of another fluorescence recording obtained several minutes after the completion of the staining procedure in the running experiment. The data presents a typical fluorescence recording of fibrillatory activity captured during ventricular fibrillation on the epicardial surface of the left ventricle of a rabbit heart. The top left panel shows the histogram of all captured intensity values within the interrogation area $\mathcal{A}$ depicted in the subgraph during the duration of the recording. The distribution of grayvalues ranges from about 5,000 intensity counts to about 30,000 intensity counts, indicating that the fluorescence is captured using about half of the 16-bit dynamic range of the camera. The range of grayvalues below 5,000 counts is not sampled as dark sites of the video frame are outside the interrogation area $\mathcal{A}$. Furthermore, the shape of the distribution comes close to a bimodal distribution with one distinct peak at about 23,000 counts and another peak at about 18, 000 counts. Indeed, examining the raw video image, one can observe that the heart's surface exhibits a particular texture, which is composed of small brighter tiles and darker thin edges surrounding these tiles. This pattern is typical and can be observed in many fluorescence recordings. The two bright and dark domains of the pattern correspond to the two peaks in the histogram. The top right panel in figure 6.9 shows two traces obtained from two pixels $\left(x_{1}, y_{1}\right)$ and $\left(x_{2}, y_{2}\right)$, which are positioned very close to each other $(d=8.06$ pixels $)$ in the video data and on the surface of the heart, see black dots in raw video image in subgraph window. The traces show fluctuating fluorescence intensity $F$ with typical characteristic rapid downstrokes indicating transmembrane depolarizations and electrical activity. In the $1.0 s$ of the recording, one can observe 8-9 action potentials, which appear synchronously in both traces. Estimates for the amplitudes of the downstrokes $\Delta F$ are indicated by 

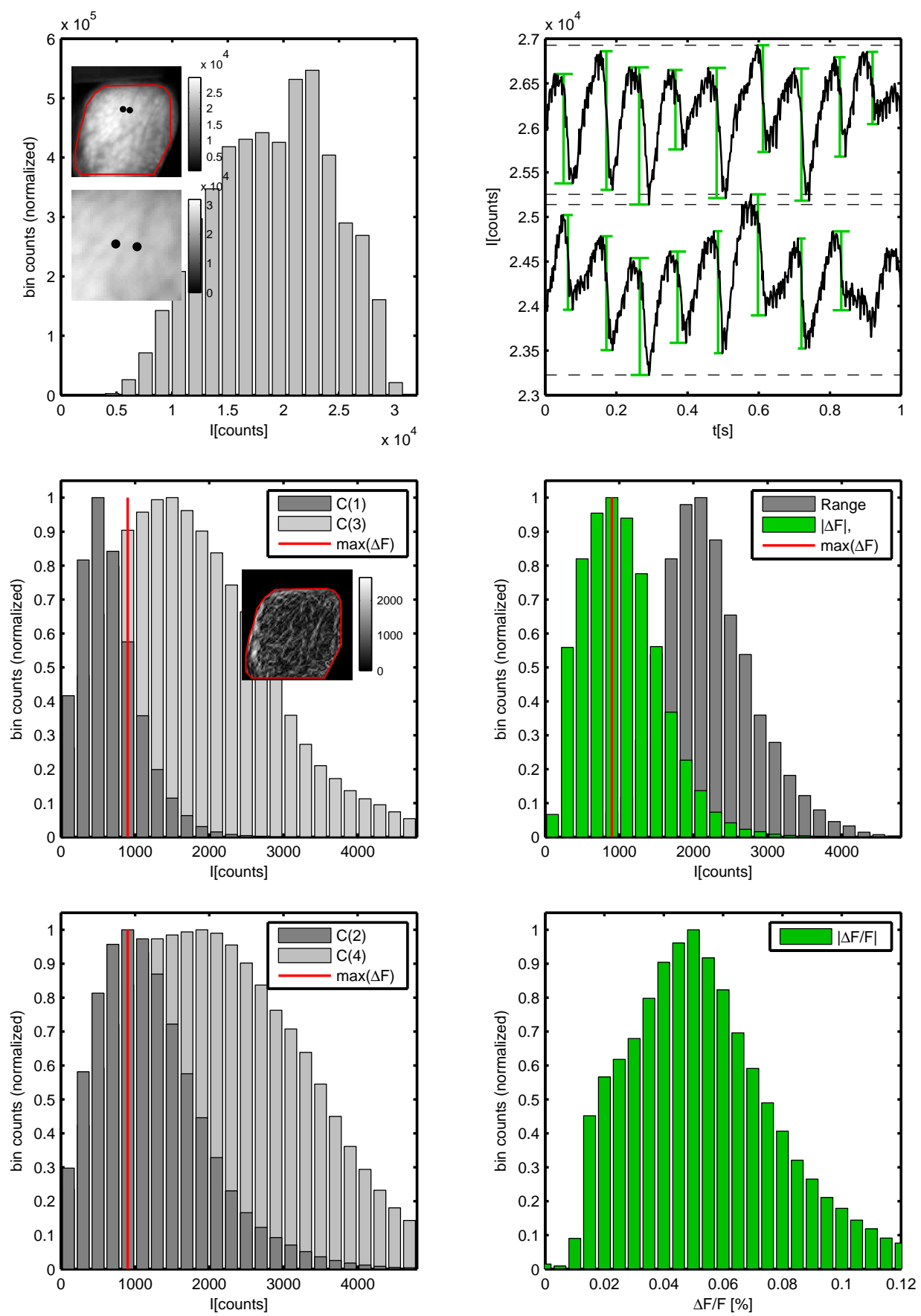

Figure 6.9: Fluorescence and image contrast of fluorescence recording showing fibrillatory activity. Top left panel: histogram of pixel grayvalues inside $\mathcal{A}$ (red area in subgraph). Top right panel: traces of two neighbouring sites depicted by black dots in subgraph in top left panel. Estimates for amplitudes of downstrokes indicated by green bars. Central right panel: histograms of $\Delta F$ and grayvalue range of the pixels. Left central and lower panel: histograms of image contrasts $C$ with varying subwindow size. Bottom right panel: fractional change of fluorescence $\Delta F / F$ with peak at $5.6 \%$. See text for further explanations. 
green bars. As previously described, the amplitudes were computed using pairs of neighbouring local maxima and minima. It is easy to note that both traces have different baselines and that the absolute difference in the two baselines is approximately larger than the intensity fluctuations $\Delta F$ of each individual trace. The difference in the baselines corresponds to the two pixels laying on different plateaus of the grayvalue texture of the image, one pixel laying inside a brighter tile and the other pixel laying on a dark edge, see lower small subwindow in the top left panel. The range of each trace is indicated by dashed lines. Next, one can observe that not only the individual downstrokes vary in magnitude but that the baseline itself contains a modulation which leads to a drift of the signal. This modulation is the contribution of motion and was considered to be a motion artifact. With the picture that the two pixels show two co-localized sites which slide in and out of each pixel's covered region during every contraction of the tissue it becomes clear that these modulations are caused by an optical flow of the texture of the image, see also next section. The central right panel shows the two histograms of once the fluorescence changes $\Delta F$ that were measured for all pixels in the interrogation area $\mathcal{A}$ as described above as well as the distribution of the grayvalue range of the traces of each of the pixels, as indicated by the dashed lines in the top right panel. The distribution of the change of fluorescence peaks at about 1,000 counts, indicated also by the red vertical bar. The distribution of the grayvalue ranges peaks at about 2,000 counts, underlining that the video contains motion which leads to an overall drift in each pixel that is significantly larger than the electrical signal. The central left and bottom left panel show the histograms of the distributions of image contrasts $C(1), C(2)$, $C(3)$ and $C(4)$ respectively, computed as described previously for the other data set, see also the contrast map in the small subwindow. For comparison, the central peak value of the distribution of fluorescence changes is indicated by the red vertical bar. Both panels together show that the magnitude of the fluorescence changes $\Delta F$ compare to the magnitude of the image contrast within a 2-pixel reach. Grayvalue changes of 2,000 counts become possible if the fluorescence change $\Delta F$ additionally experiences an intensity variation contributed by the surrounding sliding into the pixel leading to maximal possible ranges of about 2, 000 counts in that pixel. Hence, it can be assumed that the average displacement is about 2 pixels. The bottom right panel in figure 6.9 shows the histogram of the fractional change of fluorescence $\Delta F / F$ measured over all pixels laying insid the interrogation area $\mathcal{A}$. Here, the above determined $\Delta F$ were normalized by the average intensity $F$ of each pixel. The skewed shape of the distribution indicates that the method seems to overly extract smaller amplitudes. However, the method enables to determine the fractional change of fluorescence to be $\Delta F / F=5.0 \% \pm 0.5 \%$ from the peak of the histogram.

Figure 6.10 shows other typical fluorescence video data, obtained in different experiments with different rabbit hearts and dyes, and lists the parameters $\Delta F / F$ and $C / F$. In summary, the analysis demonstrates that staining has a significant effect onto the image properties of fluorescence recordings and that video data showing fluorescing cardiac tissue exhibits more contrast than video data showing simply illuminated cardiac tissue. The contrast in the images is equally large or larger than the fluorescent signal $\Delta F$. This has important implications for motion tracking and the analysis presented in the following sections.

\subsubsection{Motion Tracking and Motion Artifact Removal}

All experiments were performed without the use of pharmacological uncoupling agents, as it was desired to measure electrical activity together with mechanical activity. As a result, all fluorescence recordings presented in this work show strongly contracting and moving hearts. This experimental approach stands in strong contrast to traditional experimental protocols used in cardiac optical map- 


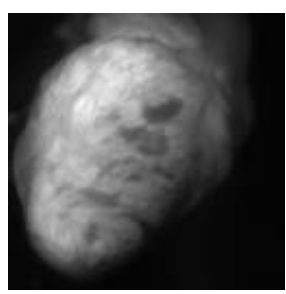

Di-4-ANEPPS

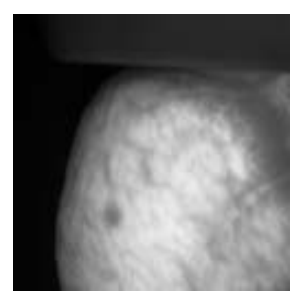

Di-4-ANEPPS

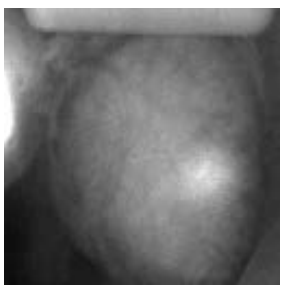

Di-4-ANBDQPQ

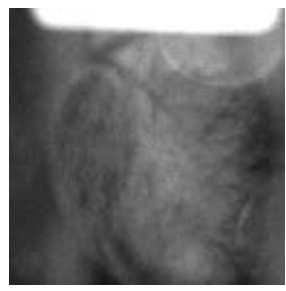

Rhod-2AM

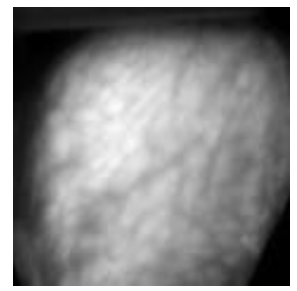

Di-4-ANEPPS

Figure 6.10: Typical examples of raw fluorescence video recordings showing the ventricle of an isolated Langendorff-perfused rabbit heart. Video still images showing fluorescence emitted from the ventricular surface captured through a bandpass or longpass filter. In all videos the image contrast is larger than the fractional change of fluorescence $\Delta F$, enabling motion tracking. The tip of the ultrasound transducer head is visible in the four right images on the top.

ping experiments, where uncoupling agents are typically used without exception in order to inhibit or entirely remove motion. The reason for this measure is to establish imaging conditions that allow the straight-forward extraction of the fluorescent signal, that is the change in fluorescence $\Delta F$ or fractional change of fluorescence emission intensity $\Delta F / F$, during the post-processing procedure. Using pharmacological uncoupling agents guarantees that the allocation of one local region of tissue emitting fluorescence with one pixel on the sensor of the camera capturing the same fluorescence remains fixed throughout the entire video, as depicted in figure 6.11. This is very convenient, as in the post-processing procedure the extraction of the fluorescent signal can be performed easily pixelbased and no further processing becomes necessary.

Here, motion tracking was performed not only for the purpose of circumventing the problems described above, but furthermore for capturing at the same time the time-varying deformation of the cardiac substrate itself. The need for computational techniques to handle motion in cardiac optical mapping experiments post-acquisition has been acknowledged ${ }^{104,122}$ several times in the literature, however, only under the aspect of aiming to inhibit or remove motion artifacts in experiments in which pharmacological uncoupling agents were already applied. In this work, tracking of the motion of cardiac tissue preparations inside the fluorescence video data is done for two reasons: one the one hand, it was desired to measure the electrophysiological activity of contracting cardiac tissue motionartifact-free. On the other hand, it was desired to measure the mechanical deformation of the muscle itself. Hence, the same method could be applied to extract two parameters of the coupled electromechanical problem at the same time. In particular, motion was not considered to be an artifact, but rather a physiological quantity closely entangled with the electrophysiology.

\section{Motion Artifacts}

Figure 6.11 illustrates how motion artifacts arise in optical mapping experiments. Traditionally, a pixel-based post-processing procedure is used for the extraction of the fluorescent signal $\Delta F$, assuming that each pixel shows the same piece of tissue emitting the fluorescence during the entire duration of the recording. Having applied pharmacological uncoupling substances, most of the time this condition is fulfilled. In the left panel, the pixel at coordinate $(x, y)$ is associated with the tissue material point $(i, j)$ and for non-moving cardiac tissue preparations this association will be maintained throughout the recording. The extraction of the fluorescent signal $\Delta F$ then simply involves to extract and normalize the time-series $F(x, y, t)$ that is obtained from the pixel $(x, y)$, for instance. 

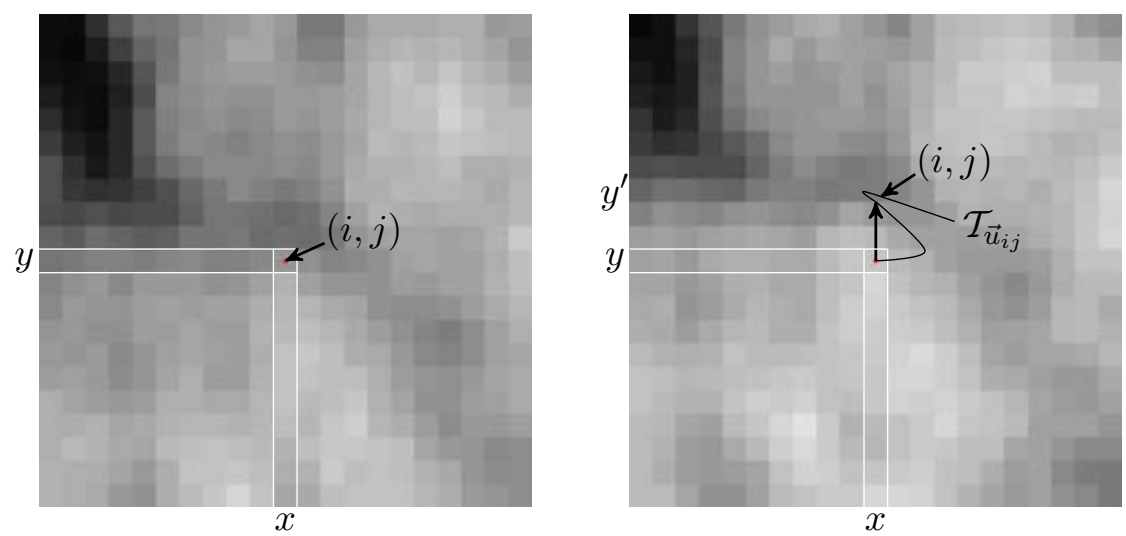

Figure 6.11: Motion artifacts in optical mapping experiments: motion causes de-allocation of tissue material point $(i, j)$ and pixel coordinate $(x, y)$. If post-processing is performed pixel-based, using the coordinate system of the detector grid, then neighbouring pixels create 'optical flow', see also figure 6.14. Motion tracking used to reconstruct trajectory $\mathcal{T}_{\vec{u}_{i j}}$ that follows tissue particle $P^{i j}$, which is initially located at $(x, y)$ and later located at arbitrary $\left(x^{\prime}, y^{\prime}\right)$. The fluorescent signal $\Delta F$ is accordingly extracted along trajectory $F\left(\mathcal{T}_{i j}(x, y), t\right)$. Image shows $\sim 1.5 \mathrm{~mm} \times 1.5 \mathrm{~mm}$ section of the left ventricular surface, see also figure 6.14.

However, as soon as the tissue moves, the grayvalue pattern slides through the pixel plane and the tissue material point $(i, j)$ can not be allocated at the coordinate $(x, y)$, but instead, will be found elsewhere at the pixel coordinate $\left(x^{\prime}, y^{\prime}\right)$, as can be seen in the right panel. The pixel at the original coordinate $(x, y)$ now shows another piece of tissue and, as a result, exhibits a modulation of its intensity that is not directly related to electrophysiological activity, but instead is caused by optical flow, that is the grayvalue pattern of the surrounding pixels sliding into the actual pixel due to motion. The time-series shown in figures 6.9 and 6.14 show typical examples of motion artifacts. The timeseries that show action potential activity additionally appear to be distorted and modulated in their baseline. In the fluorescence recordings acquired throughout this work, it was found that the average displacements of tissue in the pixel plane is typically in the order of $0.5-5$ pixels. That means, that the pixel grayvalue intensity given at the location $(x, y)$ can vary to any of the values of the grayvalues given by the pixels in the neighbourhood $\mathcal{S}(x \pm \Delta x, y \pm \Delta y)$ with $\Delta x=\Delta y \leq 5$ pixels during contractile motion. Hence, the local tissue contrast $C$ that was calculated in the previous section helps to estimate the strength of motion artifacts, as the grayvalue pattern determines the strength of the optical flow. Figure 6.9 demonstrates that the local tissue contrast in the recordings may become equally large as the overall change in fluorescence $C(2) \simeq \Delta F$ already for a neighbourhood of 2 pixels. Accordingly, contractile motion that causes average displacements $\overline{\vec{u}}$ with magnitudes greater than $|\overline{\vec{u}}| \geq 2$ pixels may superimpose the fluorescent signal exhibited by each pixel to an extent that renders the extraction of the fluorescent signal impossible.

\section{Extraction of Fluorescence from Moving and Deforming Tissues in Material Coordinates}

Tracking the tissue's motion allows to follow each material particle's motion through the pixel plane and to extract the fluorescent signal $\Delta F$ not per pixel but per tissue material coordinate $(i, j)$. In principle, the technique allows to overcome the above described problem of motion artifacts as it separates the entanglement of motion-induced intensity fluctuations from intensity fluctuations directly related to fluorescence changes caused by electrical activity. Figure 6.11 already introduced 


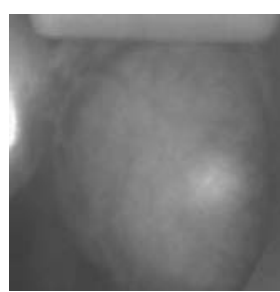

$0.0 \mathrm{~s}$

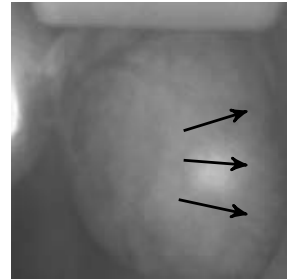

$2.2 \mathrm{~s}$

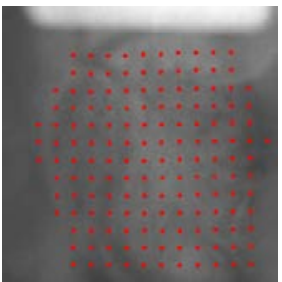

$2.2 \mathrm{~s}$

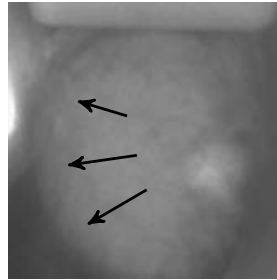

$2.4 \mathrm{~s}$

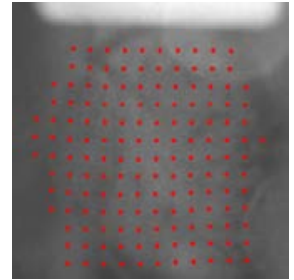

$2.4 \mathrm{~s}$

Figure 6.12: Motion tracking and reconstruction of deforming tissue configuration $\chi_{t}$ over time using tracking grid (red dots, shown every second node of $64 \times 64$ grid). Excessive motion during sinus rhythm and performance of motion tracking. The tracking grid follows the optical flow from frame to frame (inter-frame tracking).

the concept of the pixel-based allocation as well as the material coordinate $(i, j)$. In the right panel, the tissue moved upwards and the piece of tissue $(i, j)$ that was located at the pixel coordinate $(x, y)$ in the left panel can now be found at the pixel coordinate $\left(x^{\prime}, y^{\prime}\right)$. Generally, each piece of tissue $(i, j)$ moves along a trajectory $\mathcal{T}_{i j}=\left\{\vec{x}_{i j}^{0}, \vec{x}_{i j}^{1}, \ldots\right\}$ during the contractile activity of the heart, with each position given in pixels of the camera detector. Hence, extracting the fluorescence $F$ in each frame at the subsequent positions $\vec{x}_{i j}^{t}$ along the trajectory $\mathcal{T}_{i j}$ measured in pixels yields the fluorescence exhibited by each tissue material particle $F_{i j}(t)$ over time and any fluorescence change $\Delta F$ can now be attributed to be caused by electrical activity. Hence, if analyzing fluorescence recordings including motion, the post-processing procedure needs to be extended to perform the signal extraction in material coordinates of the deforming tissue.

$$
F(x, y, t) \rightarrow F\left(\mathcal{T}_{i j}(x, y), t\right)
$$

In terms of continuum mechanics, the post-processing needs to be performed in the co-moving Eulerian frame with its material coordinate $(i, j)$ (often also indicated as $(X, Y)$, see also section 2.2). This was done here. The coordinate $(x, y)$ is given in the lab coordinate, defined by the camera sensor, in the Lagrangian frame.

\section{Motion Tracking using Lucas Kanade Optical Flow Estimation and Elastic Deformation Track- ing}

Motion tracking is based on computational image registration and computer vision tracking techniques, which are able to automatically identify the same regions within an image in subsequent images of video data as they move in space, see also section 3.4.1 in chapter 3. Consequent tracking of all regions of the shown cardiac muscle captures its deformation. Respectively, tracking of all trajectories $\mathcal{T}_{i j}$ for a sufficiently large set of material pieces or all the images's pixels respectively, yields the deformation. Here, motion and deformation tracking was performed using a Lucas-Kanade optical flow estimation technique, see section 3.4.1, as well as the elastic deformation tracking technique described in section 5.5.1.

All recordings presented in this work included translational movements of the entire heart as well as deformations that appeared to occur only locally due to local contractile activity. Typically, during sinus rhythm, the motion was found to be so strong that the heart eventually partially or even entirely left the field of view. Also, rotations of the heart lead to situations, in which parts of the imaged 

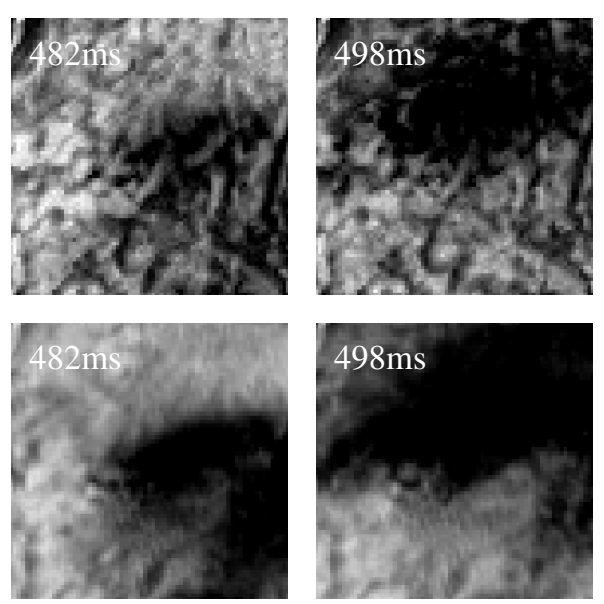
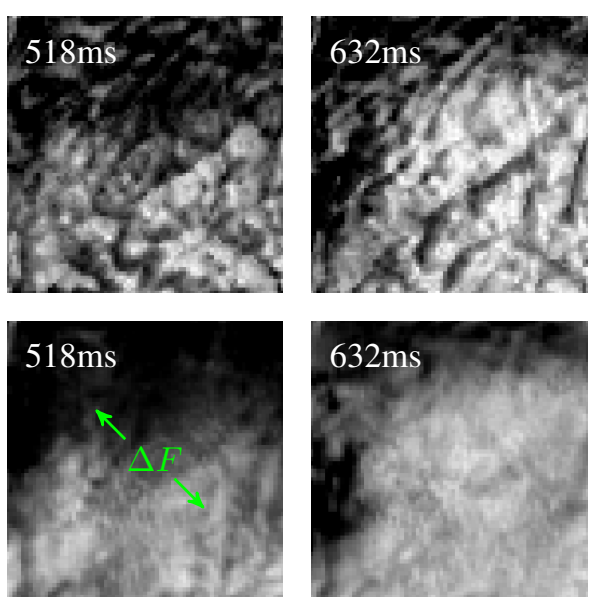

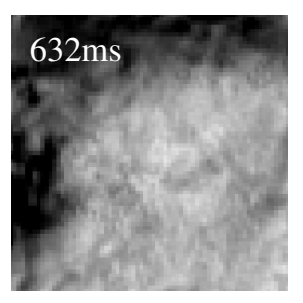

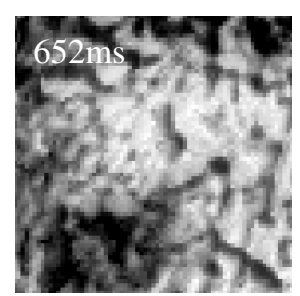

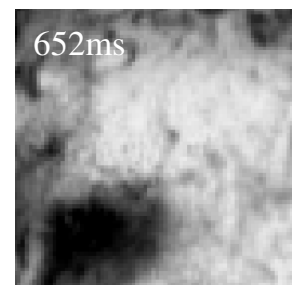

Figure 6.13: Effect of motion tracking and stabilization for motion artifact removal. Video data showing fibrillating, contracting left ventricular surface $(\sim 1 \times 1 \mathrm{~cm})$ of rabbit heart stained with fluorescent dye Di-4-ANEPPS. Upper panel: raw fluorescence video data including motion artifacts. The video was normalized in each pixel over time to intensify intensity fluctuations $\Delta I$ in the images, here seen as bright and dark areas. With motion, the intensity fluctuations are caused by (i) the surface action potential as well as (ii) the moving texture of the tissue. The large dark areas are due to the transmembrane depolarizations of the cells. In addition, the images are superimposed by an undesired small-scaled dark-bright pattern related to motion. Lower panel: tracked and stabilized fluorescence video data. Again, the video is normalized in each pixel over time. Motion artifacts are significantly reduced and the large wave pattern becomes immediately apparent.

areas facing the camera turned away, such that tracking could not be continued. In such situations, restraining the heart mechanically became necessary. During arrhythmic activity, however, the heart remained mostly in its position inside the field of view of the camera. Translational motion was low and most of the motion was found to be caused by locally contracting tissue. The fluorescence video data was tracked using inter-frame tracking as well as fixed-reference-frame tracking schemes. Figure 6.12 shows a tracking grid, set up as described in section 5.5.1, that follows the motion of the surface of the heart during sinus rhythm. The heart experiences excessive translational motion. Nevertheless, the tracking grid follows its motion from the center to the far right and then to the far left of the image. The motion was computed in this case from frame to frame using an inter-frame tracking scheme, see also section 5.5.1. Prior to tracking, the fluorescence video images were enlarged in size from $128 \times 128$ pixels to $252 \times 252$ pixels. The tracking grid consisted of $64 \times 64$ nodes, separated at a distance of 4 pixel from each other. Masking restricted the grid to the corresponding shape of the heart. Subregion window diameters were set to $d(\mathcal{S})=6$ pixels, to scan the surrounding of each node within a radius of up to $r=10$ pixels. Specifying a fixed reference frame and tracking with respect to this frame showed to be problematic and not always possible for the entirety of the acquired images within one image sequence, as it became increasingly difficult to find a correlation between the frames with increasing frame distance over time. However, the Lucas-Kanade optical flow estimation technique could only be performed using a fixed-reference frame tracking technique as it did not provide any regularization of the tracking. During tachyarrhythmias, on the other side, it was difficult to select the right reference configuration $\chi_{0}$ or starting point of the tracking, as the tissue was constantly contracting. Here, the elastic deformation tracking technique showed to have the advantage that the grid adjusted and constantly regularized and preserved the global shape of 

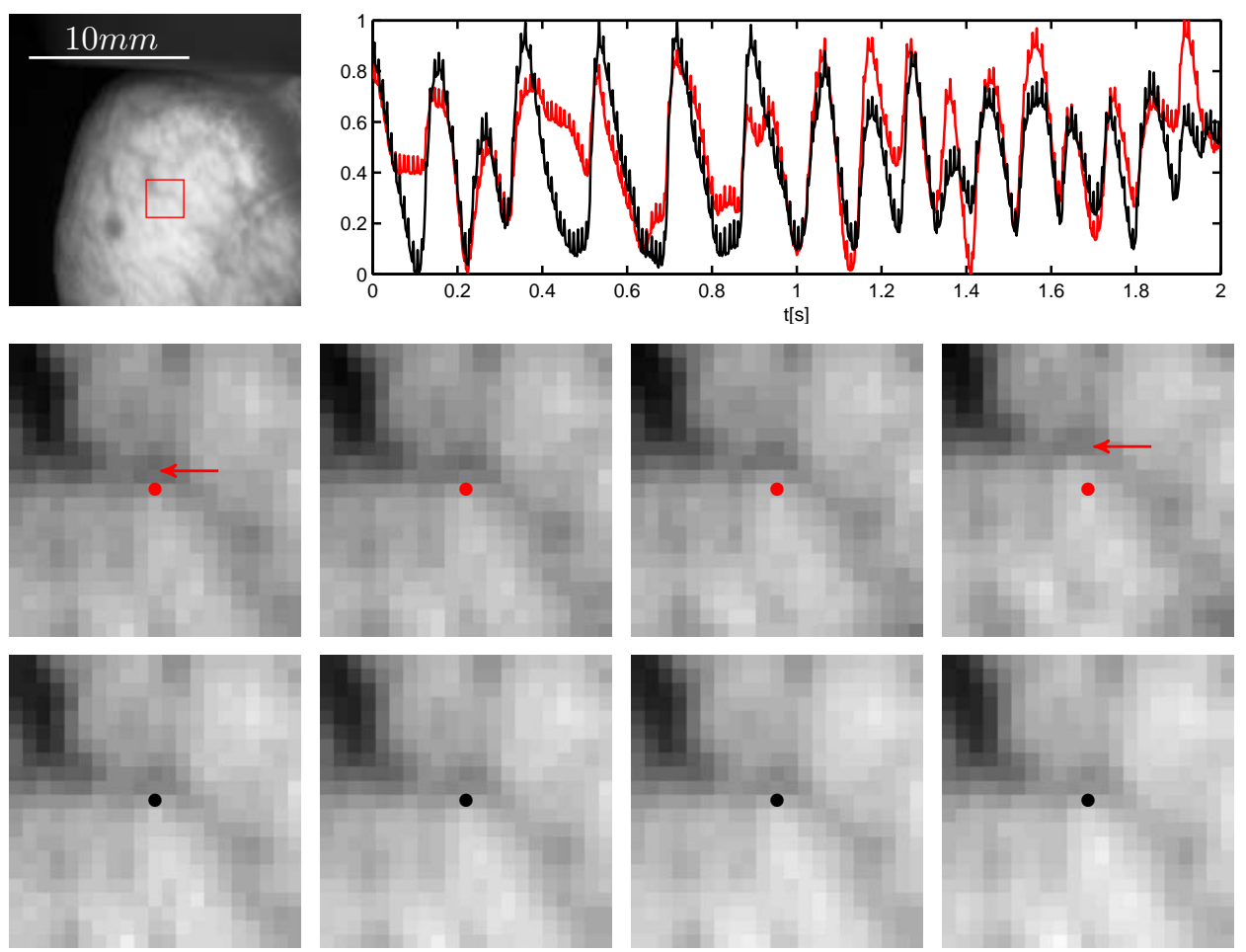

Figure 6.14: Motion tracking and warping of fluorescence recordings: tracked deformation or displacement fields used to create still movies including electrical activity and fluorescence fluctuations $\Delta F$ by warping or morphing the images showing the deforming tissue to one reference frame such that all frames show highly similar tissue configurations.

the tracking grid during the tracking process. In particular, tracking was found to yield the same or very similar results independently of the chosen starting frame. The Lucas-Kanade technique did not provide these features, as it was not intended to be used to register images in a sequence, but rather to compare only two images with each other. Hence, it was only applied using a fixed-reference frame tracking scheme.

\section{Warping}

The tracked fluorescence videos were converted into movies, in which motion was either entirely removed or at least partially inhibited. This was achieved by warping or morphing each frame using its computed deformation or displacement field to inversely deform the image, mapping it onto its respectively specified reference configuration and resampling it into a regular pixel grid raster. The resultant image sequence showed the same fluorescence video containing the fluorescence intensity variations $\Delta F$, but without motion or with singificantly reduced motion. The procedure can also be described to correspond to the visualization of the moving video in the co-moving Eulerian frame. The video data could afterwards be analyzed following the conventional, pixel-based post-processing recipes, see section 6.4.4. Figure 6.14 shows two image sequences, where one, in the upper panel, corresponds to the raw unwarped movie showing the moving tissue and the other one, in the lower panel, corresponds to the warped movie, showing the same activity but without motion. In particular, the lower panel shows frames from subsequent time steps and the video exhibits electrical activity 
if analyzed appropriately, even though it may appear that the images show four times the same still frame. The images are excerpts, approximately $1.5 \mathrm{~mm} \times 1.5 \mathrm{~mm}$ in size, from the video shown in the upper left panel, as indicated by the red box. The traces in the upper right panel are obtained from the pixel at the center of each of the images, where the red trace corresponds to the evolution of the fluorescence intensity $F(x=11, y=11, t)$ in the raw movie and the black trace corresponds to the evolution of the fluorescence intensity $F\left(\mathcal{T}_{i j}\left(x_{0}=i=11, y_{o}=j=11\right), t\right)$ in the warped movie. The red trace contains motion artifacts, whereas the black trace appears to show the same activity without artifacts. The upper sequence shows that the tissue slides upwards (red arrow) and that consequently the brighter area below the sampled pixel slides into the pixel, increasing artificially its intensity value.

\section{Image Properties and Motion Tracking}

Extracting the fractional change of fluorescence $\Delta F$ from contracting heart tissue can be particularly challenging due to several additional factors that were not as yet mentioned. For instance, it is suspected ${ }^{104}$ that, firstly, changes in the reflectance properties of the tissue may superimpose the fractional change of fluorescence as the heart deforms and that, secondly, contractile activity induces variations of local dye concentrations, which in turn modulates the intensity of the local fluorescence emission. However, it seems unlikely that an alteration of reflectance properties plays a larger role when inspecting figure 6.7 and understanding that emission light dominates the overall lighting situation. Rather, a change in illumination, as the heart moves with respect to the light sources, might play a more significant role. This may alter the exposure of tissue to excitation light and thus change the effective absorption of excitation light as well as alter the angle at which emission can leave the tissue and reach the camera. In this work, the influences of inhomogeneous illumination and dye concentrations were neglected. It was assumed that homogeneous lighting situations could be realized sufficiently well by positioning several light sources at different angles and sides of the tissue preparation, see figure 6.5. Furthermore, it was assumed that variations in dye concentration had only little impact on fluorescence emission intensity because dye attaches to fixed locations in the tissue and contractions of cardiac tissues are isovolumetric, which should limit changes in tissue and dye density.

\section{Performance}

Figures 6.13 and 6.14 demonstrate the performance of the motion tracking techniques and that they were able to extract and remove motion from the recordings succesfully. Based on visual inspection, the motion was almost entirely removed from the corresponding video and the time-series obtained from the warped movie shows significantly less motion artifact. However, it needs to be noted that the systematic evaluation of the quality of the tracking remained difficult. The performance was found to depend on the individual data set and its image properties as well as the type and strength of the contractile motion. Furthermore, it was found that motion tracking was not always necessary. For instance, during arrhythmias, when the overall translational motion of the heart was low and reduced to local contractile activity with average displacements ranging in the order of $2-3$ pixels, and the fluorescenct signal was strong $(\Delta F / F>5 \%)$, it was also possible to extract the upstroke and waveform of the action potentials without tracking the motion. Because the upstroke of the action potential occurs rapidly and the contracile motion sets in after the electromechanical delay, typically a few frames after the depolarization of the tissue, the steep upstroke could be isolated from motion artifacts by computing the derivative of the signal in each pixel. This form of analysis is feasible only 


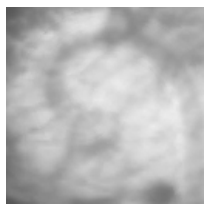

0

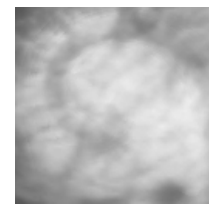

5

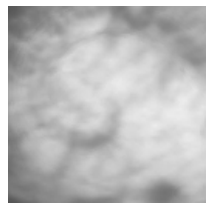

10

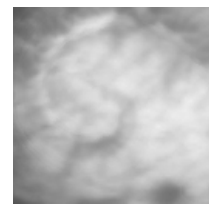

15

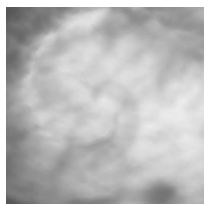

20

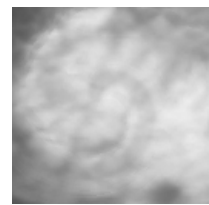

25

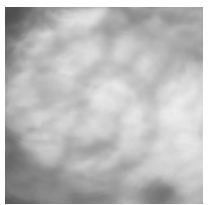

30

Figure 6.15: Synthetic fluorescence video created using a two-dimensional coupled electromechanical simulation with spiral wave activity. The simulation output deforms a fluorescence recording still frame showing the heart surface accordingly and modulates the grayvalue intensity pattern according to the underlying wave pattern. The image is rasterized and then tracked and the output of the tracking can be compared to the actual deformation field produced by the simulation. Here, the electrical spiral causes the grayvalue pattern to darken by $10 \%$ to mimick a fractional change of fluorescence of $\Delta F / F=0.1$

if one does not aim to analyze action potential durations or action potential shapes quantitatively, but instead is interested in the topology of the action potential wave pattern. Warping was found to be an effective procedure to be able to perform the post-processing of the fluorescence recordings in a quiescent frame. Even though motion could not be removed entirely from the image sequence, it was inhibited to a degree that further post-processing of the movie data became acceptable. Even though there was often still an overall drift in the data, contractile motion was reduced significantly.

\section{Validation}

Aside from visual inspection, the effectiveness and accuracy of motion registration was evaluated and validated by creating synthetic fluorescence movies using the coupled electromechanical simulation presented in chapter 4. A two-dimensional version of the simulation was used to deform a still frame from a fluorescence recording according to the electromechanical wave activity, see figure 6.15. The still frame showed the left ventricular surface with its characteristic grayvalue pattern during voltage-sensitive staining. In addition to the deformations, the grayvalue intensity of each pixel was modulated according to the underlying electrical wave activity, such that the typical 'shadow patterns' that are observed in fluorescence imaging experiments were re-created artificially by the simulation. For instance, the dynamic variable $u$ of the simulation, see section 2.1.5, was used to decrease the intensity of the pixels by a specified fractional amount, that is typically visible in optical mapping recordings. This amount was varied from $0 \%$ to up to $10 \%$ to mimick fluorecsence imaging experiments with different fluorescence signal qualities and to determine what effect the intensity fluctuations have on the accuracy of the motion tracking algorithm. The deforming image was resampled into a regular lattice pixel image data structure and stored including its 16-bit dynamic range. Figure 6.15 shows an accordingly created image series that shows a stationary spiral wave. The simulation output time steps were chosen such that one spiral rotation was covered in 60 images. The spiral wave pattern above is (barely) visible with the fractional change in intensity of the grayvalue pattern set to $\Delta F / F=0.1$. One can observe a counter-clock-wise rotating spiral wave shadow pattern. The image deforms accordingly. It was found that the motion tracking reconstructed the overall deformation accurately, but less accurately at the wave front and back as the intensity fluctuations around the boundaries of the electrical wave pattern are largest and interfere with the spatial intensity gradient of the grayvalue pattern. 

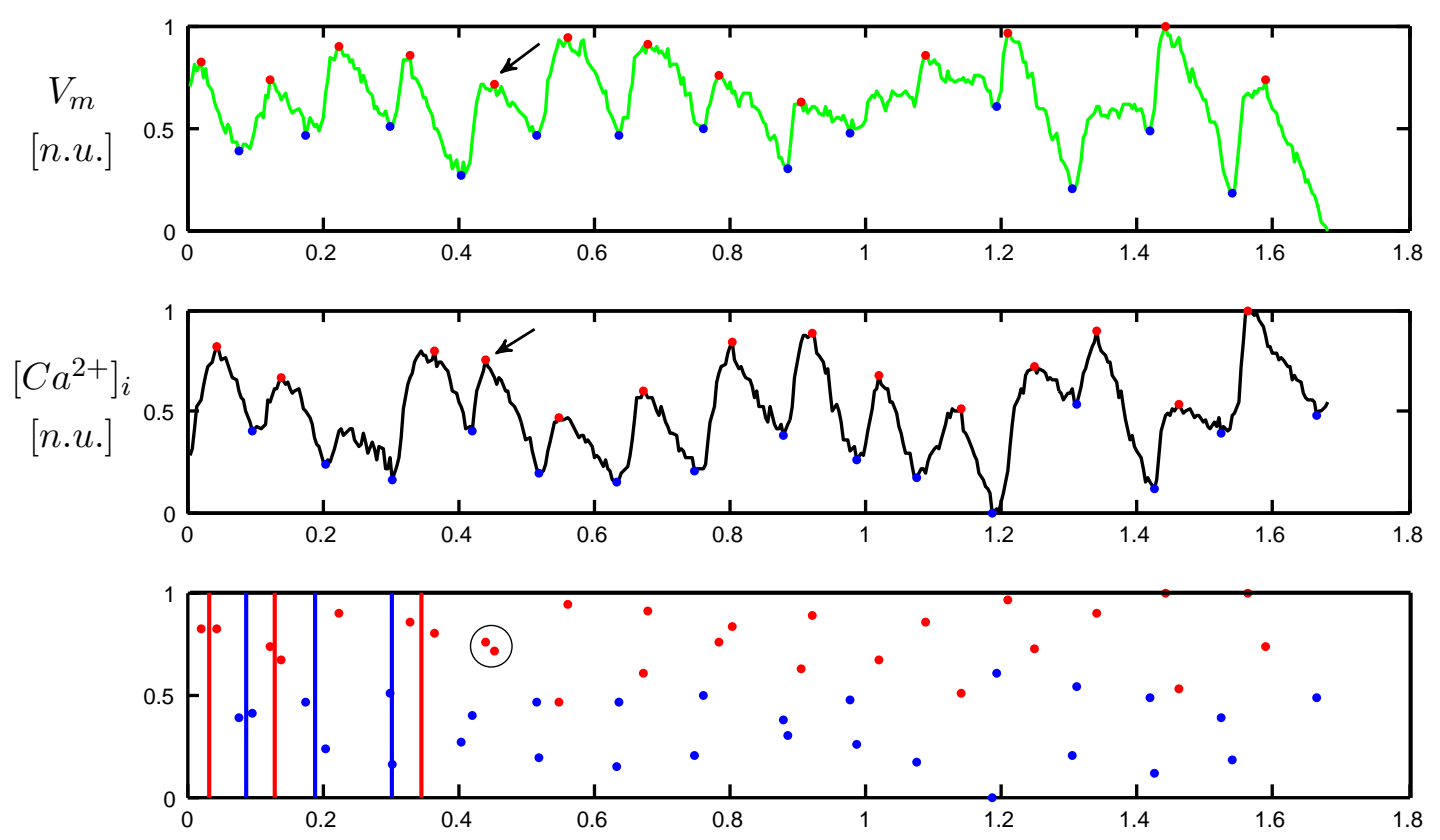

Figure 6.16: Signal-processing of optical fluorescence data including motion artifacts: raw traces from voltage-sensitive (green) and calcium-sensitive (black) videos also containing motion. Signal averaged from region (circular kernel with $r=5$ pixel) showing the same piece of tissue. Local maxima and minima detection combined with detection of upstroke yields wave fronts in both channels. Upstrokes can also be detected to occur simultaneously in both channels.

\subsubsection{Post-Processing of Fluorescence Imaging Data}

Fluorescence intensity changes were extracted depending on the type of data, either for each material point in the co-moving frame of the warped movies, or for each individual pixel respectively. To extract the wave form and propagation of the electrical action potential wave pattern, usually a series of spatio-temporal filters were applied to the movie data. The following procedure was found to produce good results: Firstly, the movie data $I(x, y, t)$ was smoothed over time and in space using spatial-temporal filtering with a small filter kernel size ( $k_{x}=k_{y}=3$ pixel, $k_{t}=3$ frames). This step reduced local high-frequency noise without altering or destroying the dynamic information carried by the fluorescent light (spatial and temporal information). Next, fast intensity fluctuations caused by the fast upstroke of the action potential wave front were intensified by computing the derivative of the fluorescent signal $\partial_{t} I(x, y, t)=I(x, y, t+\Delta t)-I(x, y, t)$ for each pixel over time ( $\Delta t=1$ frame). As a result, noisy movies showing the spread of the action potential wave front across the ventricle with dark areas corresponding to the location of the action potential wave front were obtained, see also figure 6.25. The noise was reduced by applying spatial-temporal filtering with a small filter kernel size ( for instance $k_{x}=k_{y}=3$ pixel, $k_{t}=3$ frames). Another procedure was found to produce simlarly good results: the smoothed videos were analyzed by determining the local maxima and minima in each time-series and using a spline normalization with respect to the magnitudes of the local maxima and minima, see for instance figure 6.32 to see the result. Figure 6.16 illustrates accordingly the procedure of determining the local minima and maxima. Lastly, phase representation maps $\phi\left(V_{m}\right)$ or $\phi\left(\left[\mathrm{Ca}^{2+}\right]_{i}\right)$ of the electrical patterns were created, see also section 5.3.3 in chapter 

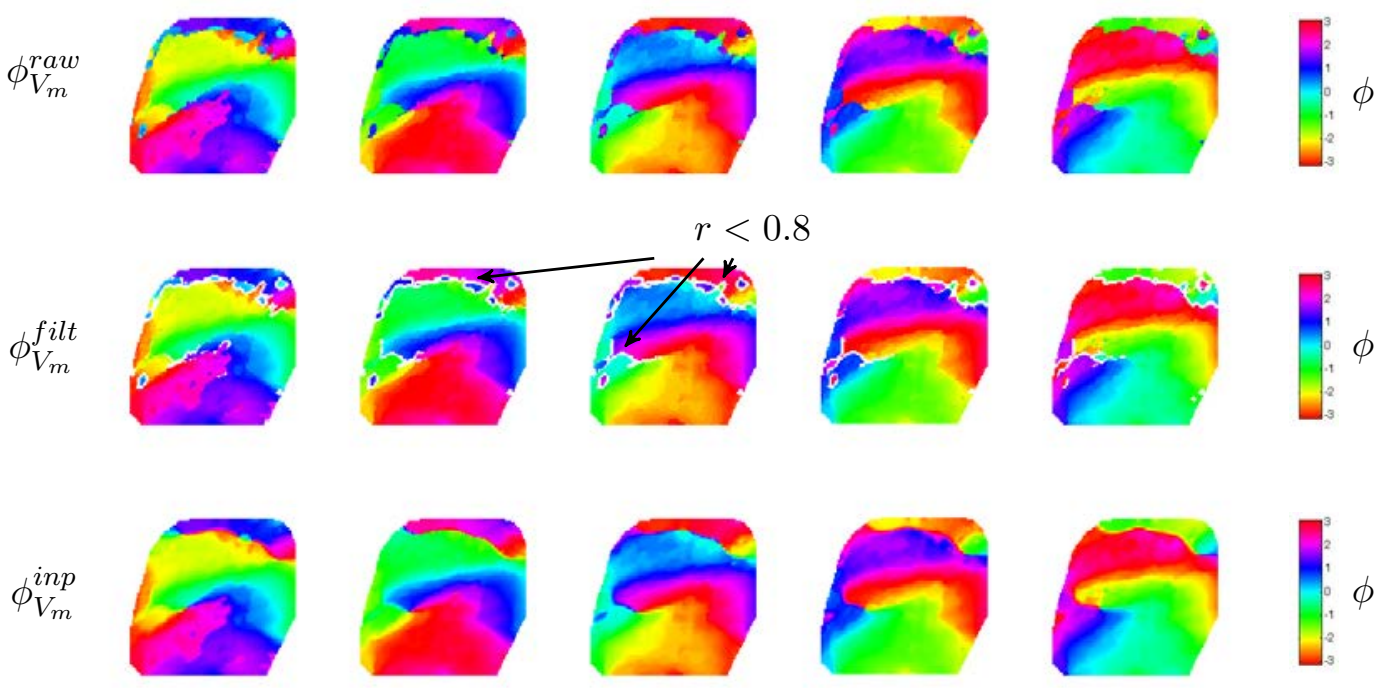

$200 \mathrm{~ms}$

$220 \mathrm{~ms}$

$240 \mathrm{~ms}$

$260 \mathrm{~ms}$

$280 \mathrm{~ms}$

Figure 6.17: Post-processing of fluorescence video data: phase representation of fluorescence videos typically contain discontinuities from the phase reconstruction procedure, computation of degree of phase coherence using order parameter of phase state angles around one pixel to identify and erase discontinuities, interpolation to obtain smoothed phase videos, see section 5.3.3.

5 and figure 6.25. The local maxima and minima were used to determine upstrokes and to introduce phase shifts in the phase maps, similarly as described in section 5.3.3. Upstrokes could also be correlated by having to occur in both electrical channels, if available, simultaneosuly. This allowed to determine the concruency of the action potential and the calcium transient wave patterns.

\subsection{Ultrasound Imaging}

Ultrasound imaging was performed using a high-speed, high-resolution ultrasound system (Vevo 2100, Visualsonics Inc., Toronto, Canada) with linear transducer array scanning head. The linear transducer array with 256 transducer elements allows to acquire two-dimensional B-mode speckle image movies at high speeds, see section 3.2. Normally, the system is used for small animal studies as an in-vivo micro imaging system. ${ }^{242}$ Here, the system was used ex-vivo to acquire speckle image movies of cross-sections of the contracting and deforming ventricular wall at high speeds with acquisition speeds ranging in the order of $\sim 300 \mathrm{fps}$ and with fields of views ranging in the order of $\sim 1 \mathrm{~cm} \times 1 \mathrm{~cm}$. Imaging provided speckle image movies, where 'speckle' refers to the speckle pattern that results from acoustic reflections of the ultrasound beam in the tissue.

\subsubsection{Imaging Setup and Data Acquisition}

The transducer $M S-550 D$, operating at a center frequency of $40 \mathrm{MHz}$ was used, as it provided the best spatial resolution and acquisition speeds. The transducer provides spatial resolutions of $13 \mu \mathrm{m}$ $/ 27 \mu \mathrm{m}$ in lateral and transversal directions respectively. To increase the imaging speed, imaging was performed at a lower line density. Overall, the system operates at an A-scan rate of $50 \mathrm{kHz}$. As each B-scan is composed of parallel A-scans that are acquired sequentially, the B-mode acquisition 
speed scales with the dimensions, more specifically the width of the image, or the number of Ascans from which the image frame is constructed correspondingly. Table B.1 shows the according B-mode acquisition speeds provided by the setup for varying image widths. Typically, imaging was performed with an image width of $9.08 \mathrm{~mm}$ or $10.08 \mathrm{~mm}$. The image depth was usually set to $12 \mathrm{~mm}$ with an offset of $2 \mathrm{~mm}$ below the transducer array, yielding an image of size $9.08 \mathrm{~mm} \times 10.0 \mathrm{~mm}$ or $10.08 \mathrm{~mm} \times 10.0 \mathrm{~mm}$. Every B-mode frame can either be acquired only once or in multiple (23) cycles to increase image quality, with the focus of the ultrasound beam set to varying positions within the image depth per cycle. To maximize imaging speed, only one focal zone was used. Gain was set to $22 d B$, power to $100 \%$ and the sensitivity was set to 'standard'. Ultrasound TTL-trigger signals were captured using a data acquisition system (MP150, Biopac Systems, Inc., Goleta, USA) and according acquisition software (AcqKnowledge, Biopac Systems, Inc., Goleta, USA), to be able to associate the start of the recording with the other acquired data, see section 6.1. Movies were acquired in raw RF-format and converted to 8-bit speckle image sequences. Image sizes were in the order of $500 \times 500$ pixel and typically reduced in size, to about $300 \times 300$ pixels, for analysis.

The imaging system provides a transducer mounting system, which allows arbitrary alignment of the imaging plane around several axes and secures the transducer in a stationary position once the desired imaging position and imaging plane alignment was found, see figure 6.5(a). Moreover, the system provides a stepping motor that can be used to perform three-dimensional volumetric measurements and can be mounted together with the transducer mounting system. The motorized translation allows to automatically acquire B-mode slices, which are acquired while the motor moves the transducer from one side to the other, and combine them together to create a three-dimensional image. The time needed for the acquisition of the sequence of images and stepping is determined by the stepping speed of the motor and ranges in the order of $\sim 10 \mathrm{~s}$. The system does not allow to set the position of the stepping motor manually. Hence, the motor was only used separately after the high-speed imaging experiments, to obtain three-dimensional scans of quiescent tissue preparations.
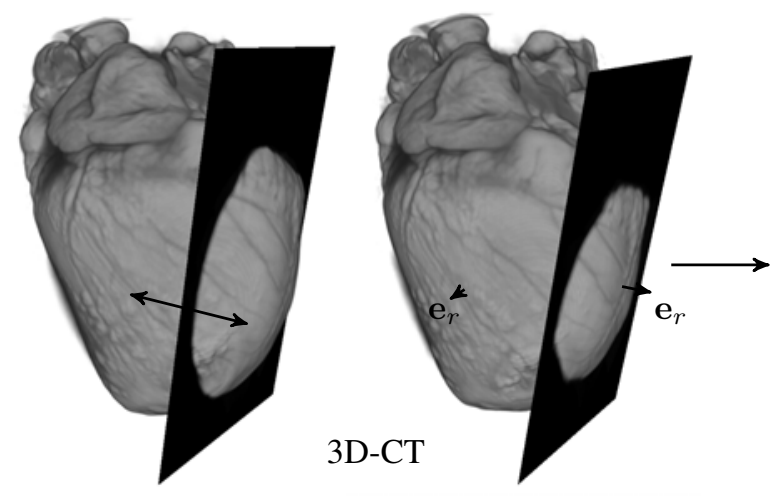

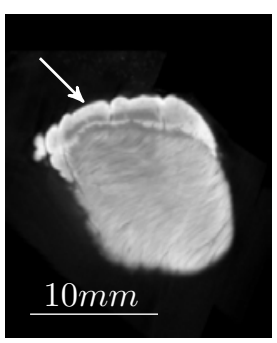

CT

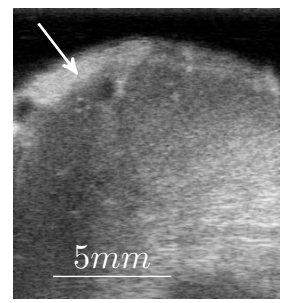

Ultrasound

Figure 6.18: Imaging configuration with two-dimensional echo-cardiographic B-mode ultrasound imaging plane: transducer positioned above heart, as illustrated in figure 6.4 (here rendering of 3D-CT scan of rabbit heart for illustration), scanning into the ventricular wall with the plane-normal pointing into the radial direction $\mathbf{e}_{r}$, see following figure. Plane positioned at midwall in parallel to both endo- and epicardial surfaces to capture cardiac motion within cross-section, see speckle image on the right. 

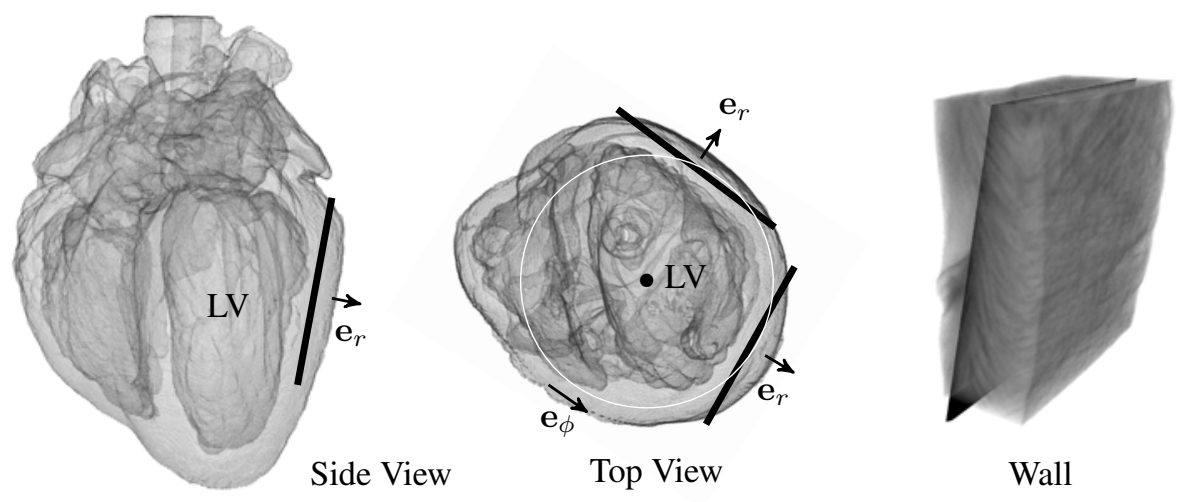

Figure 6.19: Positioning of echo-cardiographic imaging cross-sections (black lines) within the left ventricular wall of rabbit heart (here transparent rendering of 3D-CT scan of rabbit heart for illustration): in-plane vectors were thought to coincide with muscle fiber alignment. Circumferential or tangential vector $\mathbf{e}_{\phi}$ approximately coincides with tangentially aligned imaging planes. Right image: close-up of ventricular wall depicting muscle fiber sheet organization (CT-scan with specific staining technique for visualizion of muscle fiber anisotropy, see section 4.4). Simultaneous optical mapping of the corresponding ventricular surfaces using fluorescence imaging.

\subsubsection{Imaging Configuration}

Hearts were imaged with the imaging plane intersecting the left ventricular wall at midwall, as indicated in figures $6.18,6.19$ and 6.4. The plane was aimed to be embedded entirely within the wall in order to maximize the imaged volume within the two-dimensional imaging plane, and to have one of the in-plane orientations to coincide with the average muscle fiber alignment. The organization of the muscle fiber alignment inside the ventricular wall is highly complex, see section 1.2. Muscle fibers are organized in sheets, which are stacked and aligned orthotropically to form a helical laminar structure throughout the entire heart. ${ }^{18,222}$ Here, it was assumed that the laminar muscle fiber alignment follows the outer shape of the heart walls, and that the myofilaments are always aligned in parallel to the endo- and epicardial surfaces, such that each sheet-normal vector within the stack of muscle fiber sheets that is making up the ventricular wall coincides with the radial vector $\mathbf{e}_{r}$ of the heart, see figure 6.19. Consequently, it was thought that by aligning the echo-cardiographic imaging cross-section along the circumferential, tangential direction $\mathbf{e}_{\phi}$ of the heart, most contractile motion would be captured to occur within the plane, see figure 6.19. The right image in figure 6.19 shows a close-up of the ventricular wall. As the heart was prepared using a specific staining technique to add contrast to muscle fiber anisotropy, see section 4.4, the image depicts the muscle fiber sheet organization within the ventricular wall. Muscle fiber orientations can be thought of to point into and out of the cut and muscle fiber sheet orientations to intersect the black cross-section. The echocardiographic cross-sections were positioned in front of the camera, see figures 6.3 and 6.4, such that its plane normal coincided with the optical axis of the fluorescence imaging setup.

\subsubsection{Elastic Deformation Tracking}

Motion and deformations were extracted from the speckle image sequences using the motion and elastic deformation tracking techniques described in section 5.5.1. Generally, motion was registered using spatial correlation techniques, analyzing the two-dimensional pattern of acoustic reflections 

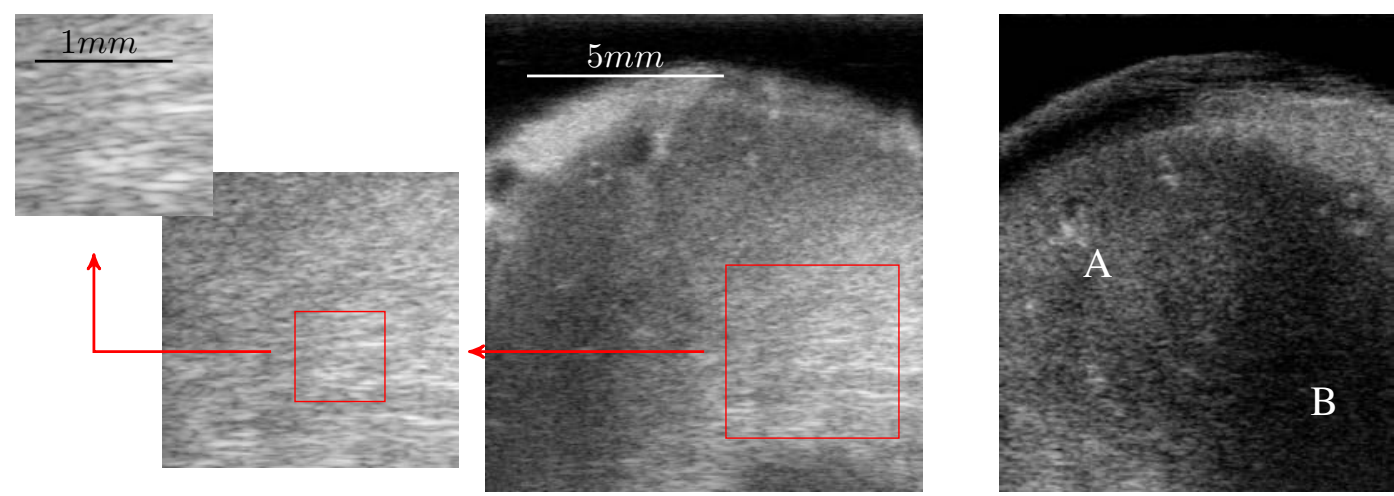

Figure 6.20: Ultrasound raw speckle image data: good quality speckle supporting motion tracking in left image. Speckle morphology typically stayed similar over 10-20 frames during arrhythmic activity. Sizes of the search kernels used during the tracking procedure typically ranged in the order of the size of the left red rectangle. Right image shows regions with good contrast (A) and poor contrast (B) due to attenuation of the ultrasound beam, the regions exhbiting poor contrast did not allowing tracking.

resulting from the ultrasound imaging process. This process is comparable to speckle tracking. The elastic deformations were reconstructed accordingly by using the motion tracking output to deform an elastic model, see section 5.5.1.

\section{Tissue Motion Registration and Elastographic Imaging}

The spatial correlation measure given by equation 5.5.2 showed to be very robust in identifying spatial shifts within the noisy ultrasound speckle images. Tissue motion was registered within speckle images of size $\sim 500 \times 500$ pixels. The subregions $\mathcal{S}$, that are the search kernels based on which the spatial correlation and similarity calculations were performed, were chosen to be of size $20 \times 20$ to $40 \times 40$ pixels, tracking structures within the speckle pattern of size $\sim 0.4 \mathrm{~mm}-0.8 \mathrm{~mm}$, see figure 6.20. Local displacements were registered using square-shaped subregions as well as circularlyshaped subregions. Motion tracking was performed, most of the time and in particular for speckle image movies showing arrhythmic activity, using an inter-frame tracking scheme, see section 5.5.1. During inter-frame tracking, the local displacements from frame to frame were found to be small with sub-pixel to a few pixel displacements, with magnitudes ranging at $\left|\vec{u}_{x, y}\right| \simeq 0-3$ pixels. The interrogation window size was adjusted accordingly to perform the search within a radius of up to $r=10$ pixels. Displacements could be tracked reliably. Figure 6.20 shows typical speckle image data obtained in the experiments. The images show cross-sections of the left ventricular wall.

Tracking was performed in the Eulerian co-moving and deforming frame. The tracking grid moved in space and deformed accordingly. The nodes between the grid were separated by a distances of 4 to 8 pixels respectively, yielding tracking grids of size $\sim 50 \times 50$ to $120 \times 120$ nodes. At each subsequent time step, the new position $\vec{x}_{i j}^{t+1}$ of each node was set to correspond to the previous position shifted by the previously calculated displacement, however, using the elastic deformation tracking technique described in the previous sections and leting the grid converge towards the tracked, noisy configuration, $\tilde{\chi}_{\vec{u}}$, such that the tracking grid followed the motion. 
Chapter 6. Intramural Scroll Wave Imaging during Ventricular Tachycardia and Fibrillation

Figure 6.20 shows typical raw speckle image movie data obtained during the ultrasound imaging experiments. The image on the left sides shows movie data with a good quality speckle pattern that supported motion tracking and was ideal for processing. The speckle pattern was distributed evenly and noise was minimal. The speckle morphology typically stayed similar over 10-20 frames during arrhythmic activity. The tracking was found to produce the same results when the initial positioning of the tracking grid was slightly changed or the initial frame was slightly shifted forwards or backwards in time. In other recordings, see image on the right, the speckle pattern appeared to be more heterogeneous, with large regions in the image exhibiting highly noisy, low contrast patterns due to attenuation of the ultrasound beam. Accordingly, the spatial correlation techniques used did not accurately find spatial displacmenets in between frames any longer, such that parts of the tracking grid experienced erratic displacements. The erratic displacments were removed and the elastic grid did not receive input from these erratically tracked displacement vectors accordingly. Out-of-plane motion occured during sinus activity of the heart, during the onset of contraction and in very strongly contracting hearts.

\section{Post-Processing}

The tracked deformations were stored as two-dimensional time-varying vector fields. During the post-processing procedure the vector fields were filtered using spatio-temporal (kernel sizes $k_{x}=$ $k_{y}=10$ pixels diameters, $k_{t}= \pm 2$ frames), their respective derivative was computed and filtered again using the same kernel sizes. Lastly, the strain rate was computed. The strain-rate pattern was converted to its respective phase representation $\phi_{\hat{E}}(x, y, t)$, similarly as described in section 5.3.3.

\subsection{Electromechanical Scroll Wave Imaging during Ventricular Tachy- cardia and Fibrillation}

Here, it is shown that rotating elasto-mechanical deformation patterns can be visualized during arrhythmic, fibrillatory activity of the heart on the ventricular surface as well as inside the ventricular wall, when capturing and analyzing the elastic deformations of the heart muscle at high speeds. The mechanical patterns were found to be highly correlated with spiraling electrical action potential wave patterns that were simultaneously observed on the surface. The spatial-temporal patterns of both the electrical and mechanical activity exhibit rotating patterns. The dominant frequencies of both patterns as well as of the electrocardiogram match during these episodes. At times, the rotational centers of the patterns appear to be co-localized. The elastic deformation tracking techniques described in the previous sections showed to be effective in tracking motion in both the fluorescence as well as in the ultrasound recordings.

Ventricular tachycardia and fibrillation was induced with rapid pacing using a bipolar or MAP electrode, see section 6.3 and figure 6.5(c), applying series of pacing stimuli at frequencies of $10-50 \mathrm{~Hz}$ to the ventricular surface. Hearts were imaged using fluorescence and ultrasound imaging simultaneously. Electrocardiograms and pacing and defibrillation pulses were recorded accordingly. Electrical spiral wave activity was observed on the ventricular surface during polymorphic tachycardia as well as fibrillation. Polymorphic tachycardia in the healthy, isolated rabbit heart is thought to be the result of either a single meandering scroll wave or paired scroll wave rotors, so-called figure-of-eight patterns. ${ }^{44}$ Here, data containing both figure of eight-patterns as well as spiral wave patterns with single spirals appearing intermittently on the ventricular surfaces during otherwise fibrillatory and 

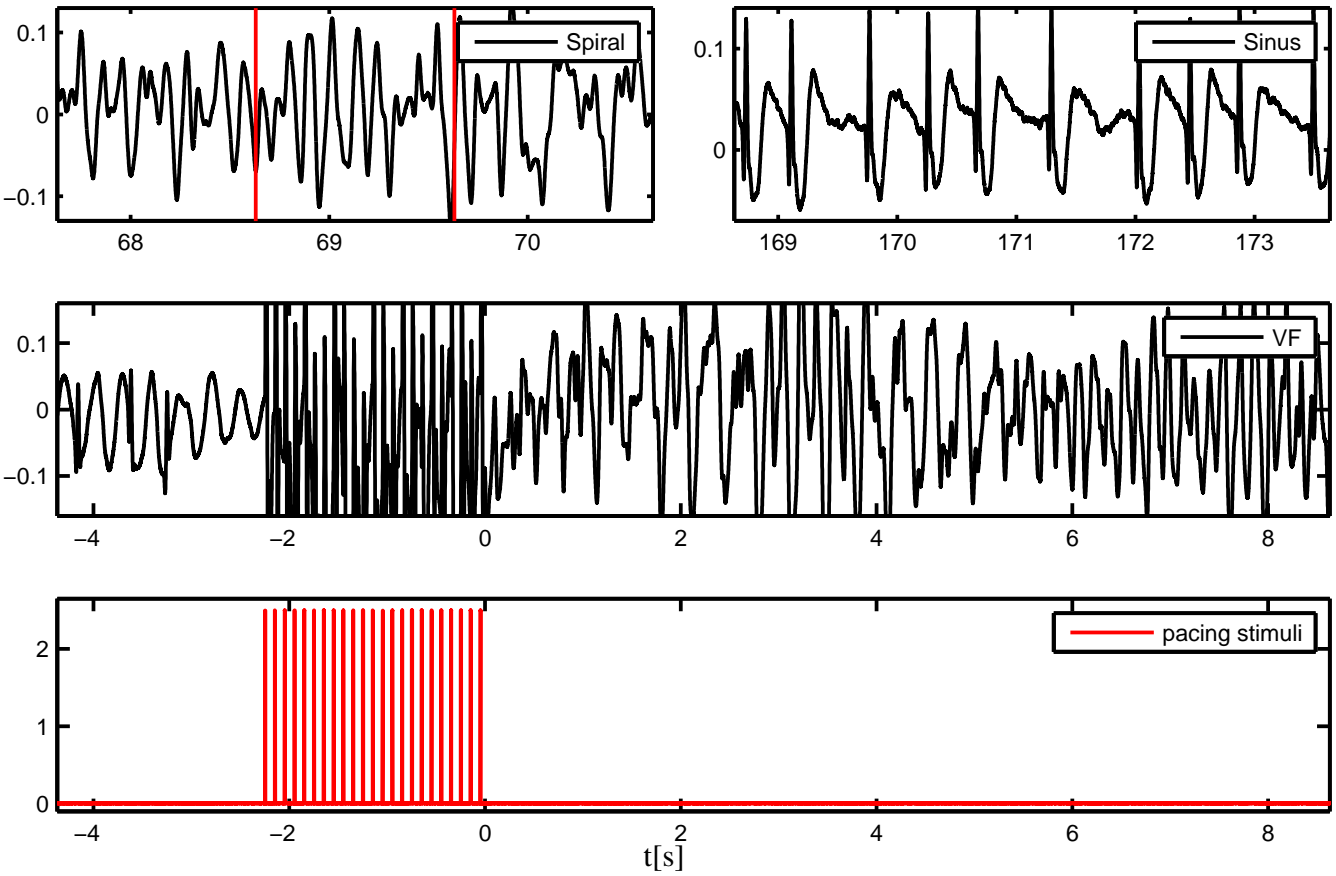

Figure 6.21: Ventricular fibrillation in rabbit heart: electrocardiogram showing highly irregular electrical activity after induction of ventricular fibrillation. Two lower panels show the induction procedure with pacing stimuli (red). The upper left panel shows a short episode after about $70 \mathrm{~s}$ in which one large counter-clock-wise rotating action potential spiral wave rotor can be observed on the left ventricular surface. The upper right panel shows the recovered sinus rhythm after low-energy anti-fibrillation pacing.

more chaotic electrical activity is presented. The rapid pacing from one site was found to induce both large-scaled rotor activity as well as highly disorderd, small-scaled fibrillatory activity, with the likeliness of the outcome varying from experiment to experiment. Large-scaled arrhythmic activity was preferrably imaged, as it was thought that this type of data was easier to interpret and analyze. Generally, however, pacing from one site was found to induce far less disordered arrhythmic activity than applying the same series of pacing stimuli via the far-field paddle electrodes. Rabbit hearts showed to be very robust against pacing in the first $30-45 \mathrm{~min}$ of the experiment. That is, the arrhythmic activity that was induced in the beginning of each experiment was often unsustained. Typically, good recordings were obtained in this time frame. More specifically, movies were acquired intentionally during the transition from arrhythmic to sinus activity, to increase the likeliness of filming largescaled rotor activity during the wash-out of fibrillation.

In the following it is shown that, first, the mechanical deformation of the ventricular surface evolves correspondingly with the electrical activity on the surface and secondly that similarly the mechanical activity below the surface evolves correspondingly with the electrical activity on the surface.

\subsubsection{Spiraling Electromechanical Activity on Ventricular Surface}

The following data set demonstrates that electrical action potential spiral wave patterns that are observed on the surface of the ventricles using fluorescence imaging, lead to deformations of the ventric- 
ular muscle that, when tracked and analyzed accordingly, exhibit similarly rotating elasto-mechanical rate of deformation patterns on the surface. The rotational patterns can be visualized when tracking the deformations of the surface as described in section 5.5.1 and following the analysis presented in chapter 5. Moreover, it is shown that the rotational centers of both the electrical action potential spiral wave pattern and the rotating mechanical pattern appear to be co-localized.

Figure 6.21 shows the electrocardiogram recorded during induced ventricular fibrillation, about 3 minutes after the start of the experiment and about 10 minutes after staining of the heart. Ventricular fibrillation was induced with a series of pacing stimuli (red bars, lower panel) applied for a duration of about $2 s$. The following fibrillatory activity shows to be highly irregular, see central panel. The upper left panel shows the electrocardiogram of an episode recorded at about $68-70 \mathrm{~s}$ after the induction of the arrhythmic activity, in which a large single spiral wave appears intermittently in between the otherwise highly chaotic activity on the left ventricular surface, see following figures. The red bars indicate the start and the end of the 1.0s long video sequence, see figures 6.22-6.23, which consists of 500 frames, each frame acquired every $2 m s$ accordingly. The overall arrhythmic episode was terminated after 2 minutes using low-energy anti-fibrillation pacing (LEAP), consisting of 5 pulses with pulse widths of $5 \mathrm{~ms}$ and cycle lengths of $80 \mathrm{~ms}$ each at an electric field strength of $70 \mathrm{~V}$, see also section 6.3. The upper right panel shows the recovered sinus rhythm accordingly.

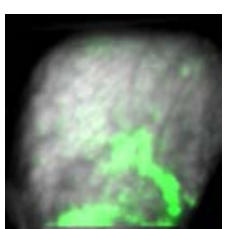

$230 \mathrm{~ms}$

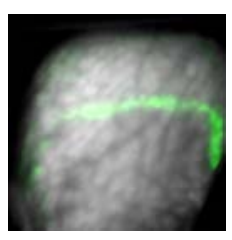

$266 m s$

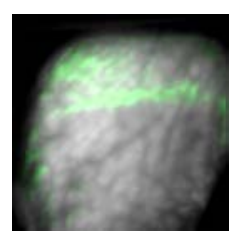

$276 m s$

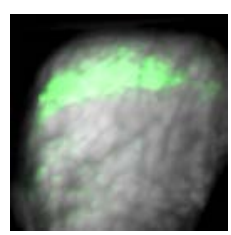

$290 \mathrm{~ms}$

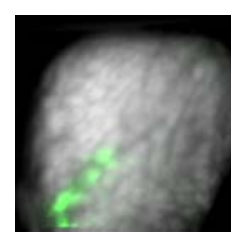

$326 m s$ exc.

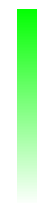

refr.

Figure 6.22: Counter clock-wise rotating action potential spiral wave (green) on left ventricular surface of rabbit heart during ventricular fibrillation. Imaged using voltage-sensitive dye Di-4-ANEPPS, which exhibits fluorescence fluctuations $\Delta F \sim V_{m}$ that enable visualization of electrical wave pattern. Rotor performed 4-5 rotations with rotational periods of $T_{A P} \approx 120 \mathrm{~ms}$.

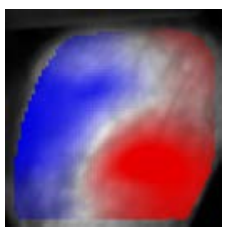

$230 \mathrm{~ms}$

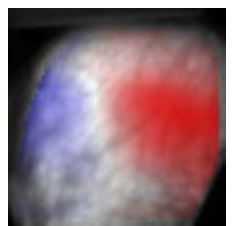

$266 m s$

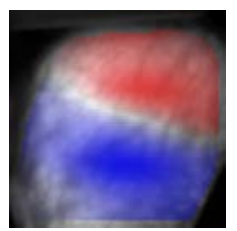

$276 m s$

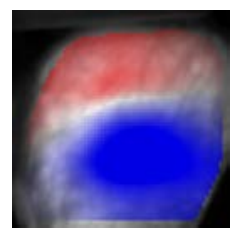

$290 \mathrm{~ms}$

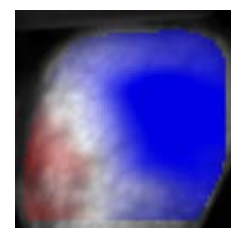

$326 \mathrm{~ms}$

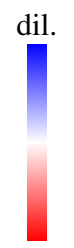

contr.

Figure 6.23: Counter clock-wise rotating elasto-mechanical rate of deformation pattern (dilating, contracting) on left ventricular surface of rabbit heart induced by electrical spiral wave activity shown above. Deformations tracked using elastic deformation tracking, see section 5.5.1, contractile motion induced by the spiral wave shown above.

Figure 6.22 shows the action potential spiral wave rotor that appeared on the left ventricular surface during fibrillation. The rotor emerged spontaneously from chaotic action potential wave patterns on the side of the heart facing the camera and performed 4-5 counter-clock-wise rotations at rotational periods of $T_{A P}=123 \mathrm{~ms} \pm 10 \mathrm{~ms}$, with its core being positioned approximately within the central 
part of the field of view. In the meantime, the rotor almost entirely displaced all other electrical activity from the field of view, occupying large parts of the left ventricle. During the fifth rotation, the rotor degenerated into a pattern composed of multiple smaller spiral waves and the following activity showed to be, as before, highly irregular. Comparing the activity with the electrocardiogram, it seems that each rotation corresponds to one of the large spikes, see top left panel in figure 6.21. The action potential wave pattern was imaged with fluorescence imaging, in mono-parametric imaging mode, using voltage-sensitive dye (Di-4-ANEPPS) that exhibits fluorescence fluctuations in dependence of the transmembrane potential $\Delta F \sim V_{m}$, which enables the visualization of the electrical pattern as seen above. The raw fluorescence movie data $F(x, y, t)$ was masked and then filtered using spatialtemporal filtering (kernel sizes $k_{x}=k_{y}=3$ pixels diameter, $k_{t}= \pm 3$ frames), before computing the derivative $\partial_{t} F$ of the signal, see also section 6.4.4. Traces obtained from single pixels of the raw video are shown in the upper right panel in figure 6.9 in section 6.4.2.

Figure 6.23 shows the accordingly imaged and tracked deformation of the ventricular surface. The deformation was tracked within the same video as shown above, using the elastic deformation tracking techniques described in section 5.5.1. The contractile motion and deformation of the cardiac muscle visibly exhibits torsional, rotational movements. The extracted time-varing rate of deformation pattern, derived from the Green-Lagrangian deformation tensor $\mathbf{E}$, see section 5.1.1, shows rotating patterns of periodically dilating and contracting regions, that seem to rotate around the central part of the field of view, and share the same rotational sense and angular speeds with the action potential spiral wave rotor. The rotational period of the mechanical pattern $T_{\hat{E}}=109 \mathrm{~ms} \pm 10 \mathrm{was}$ found to be similar with the rotational period of the action potential spiral wave. The motion was tracked using a tracking grid of size $64 \times 64$ nodes, separated at a distance of 4 pixel from each other. Prior to tracking, the fluorescence video images were enlarged in size to $252 \times 252$ pixels. Subregion window diameters were set to $d(\mathcal{S})=6$ pixels, to scan the surrounding of each node within a radius of up to $r=4$ pixels. The resulting strain pattern $\hat{E}$ was filtered using spatial-temporal filtering (kernel sizes $k_{x}=k_{y}=14$ pixels diameter, $k_{t}= \pm 10$ frames), before computing the derivative $\partial_{t} \hat{E}$ of the signal, and smoothing the resultant strain-rate pattern again using the same spatial-temporal filtering, see also section 6.5.3.

Figure 6.24 shows the frequency spectra of the mapped electrical action potential spiral wave pattern, the tracked mechanical deformation pattern, shown in figures 6.22 and 6.23, and of the electrocardiogram. All spectra appear to be monochromatic with one pronounced peak at around $9.0 \mathrm{~Hz}$ $\left(f_{A P}=8.1 \mathrm{~Hz} \pm 1.1 \mathrm{~Hz}, f_{\hat{E}}=9.1 \mathrm{~Hz} \pm 1.1 \mathrm{~Hz}, f_{E C G 1.0 \mathrm{~s}}=9.0 \mathrm{~Hz} \pm 1.0 \mathrm{~Hz}\right.$ and $f_{E C G 1.0 \mathrm{~s}}=$ $8.9 \mathrm{~Hz} \pm 0.1 \mathrm{~Hz}$ ). This indicates that all three quantities are closely related and suggests that the two electrical and mechanical patterns are two different manifestations of the same underlying electromechanical scroll wave rotor. The frequency spectra of the two-dimensional patterns were computed as the cumulative power spectra of the single traces of the individual pixels. Note, that the sampling duration of $1.0 \mathrm{~s}$ is very short for the computation of the power spectra. Their resolution is accordingly low. However, due to the monochromaticity of the dynamics the resultant $\pm 1 \mathrm{~Hz}$ uncertainty was found to be acceptable. The frequency spectra of the electrocardiogram was computed for the $1.0 \mathrm{~s}$ duration of the recording as well as for a $10.0 \mathrm{~s}$ time frame around the recording, to increase the resolultion. 

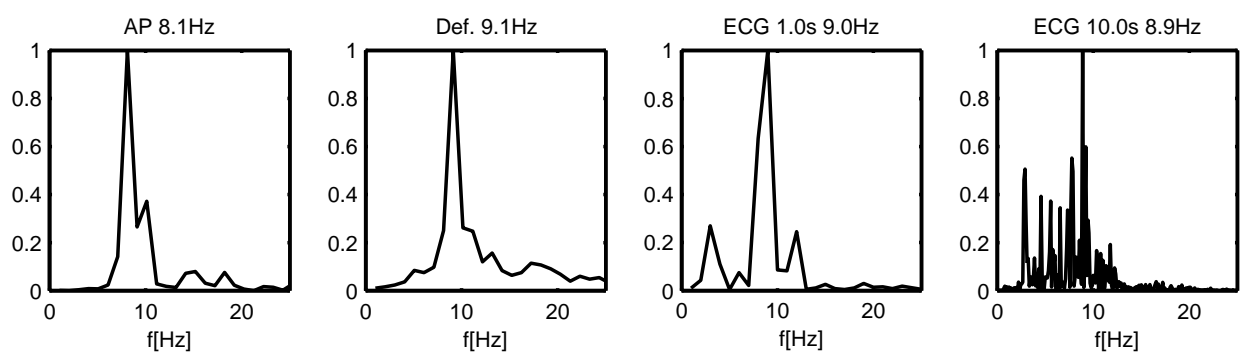

Figure 6.24: Frequency spectra of epicardial action potential spiral wave pattern (AP with $f_{A P}=8.1 \mathrm{~Hz} \pm$ $1.1 \mathrm{~Hz}$ ), elasto-mechanical rate of deformation pattern (Def. with $f_{\hat{E}}=9.1 \mathrm{~Hz} \pm 1.1 \mathrm{~Hz}$ ), see corresponding figures 6.22 and 6.23, and the electrocardiogram for durations of $1.0 \mathrm{~s}$ and $10.0 \mathrm{~s}$ respectively $\left(f_{E C G 1.0 s}=9.0 \mathrm{~Hz} \pm 1.0 \mathrm{~Hz}\right.$ and $\left.f_{E C G 1.0 \mathrm{~s}}=8.9 \mathrm{~Hz} \pm 0.1 \mathrm{~Hz}\right)$. All spectra appear to be monochromatic and indicate a dominant frequency of the underlying dynamics of about $9 \mathrm{~Hz}$.

\section{Reconstruction of Rotational Wave Pattern Topolgy}

Following the analysis presented in section 5.3.3 in chapter 5, the phase representations of the electrical action potential spiral wave pattern $\phi(x, y, t)_{A P}$ and of the elasto-mechanical rate of deformation pattern $\phi(x, y, t)_{\hat{E}}$ were derived. Figure 6.25 shows the conversion of the electrical and elasto-mechanical signals into continuous phase-mapped time-series, examplary for one point on the right central part of the two rotating patterns located at pixel $\vec{x}=(80,60)$. The $2 \pi$ phase shifts wer introduced, for the electrical signals, at the upstroke of the action potential, which corresponds to the negative peaks in the derivative $\partial_{t} F$ shown in the upper panel in the figure, and, for the elastomechanical signals, at each transition from dilating (blue) to contracting (red) rates of deformation, shown in the lower panel of the figure.

Figures 6.26 and 6.27 show the corresponding phase-mapped fields $\phi_{A P}(x, y, t)$ and $\phi_{\hat{E}}(x, y, t)$, with the phase state being displayed using a continuous HSV-colorcode ranging from $-\pi$ to $\pi$ to facilitate viewing of the rotor topology, compare also with figure 5.8 in chapter 5 . The patterns rotate approximately in phase with a slight delay of the mechanical pattern, see $t=322 \mathrm{~ms}$, which presumably is related to the electromechnical delay. Both rotating patterns similarly allowed to compute phase singularities (black dots), which indicate the rotational centers of the patterns. Similarly, as described in chapter 5, the phase singularities mark, in the electrical picture, the action potential spiral wave core, and, in the mechanical picture, its respective mechanical counterpart, a topological defect within the dynamically changing, rotational deformation pattern. However, the elastic pattern appeared to be more volatile, with the phase singular points moving in and outside of the field of view, splitting up into pairs or annihilating, while the rotational outer pattern persisted, as seen for $t>320 \mathrm{~ms}$.

\section{Co-Localization}

Figure 6.28 shows two snapshots from the series shown above at $t=318 \mathrm{~ms}$ together with one image of the imaged ventricular surface and the locations of electrical (green) and mechanical (red) phase singularities, that were tracked within a $\sim 110 \mathrm{~ms}$ period starting from $208 \mathrm{~ms}$ to $322 \mathrm{~ms}$ in the recording, which approximately corresponds to one spiral rotation. Within that time frame, both phase singularities were found to be co-localized, with the average postion of the electrical 
Chapter 6. Intramural Scroll Wave Imaging during Ventricular Tachycardia and Fibrillation
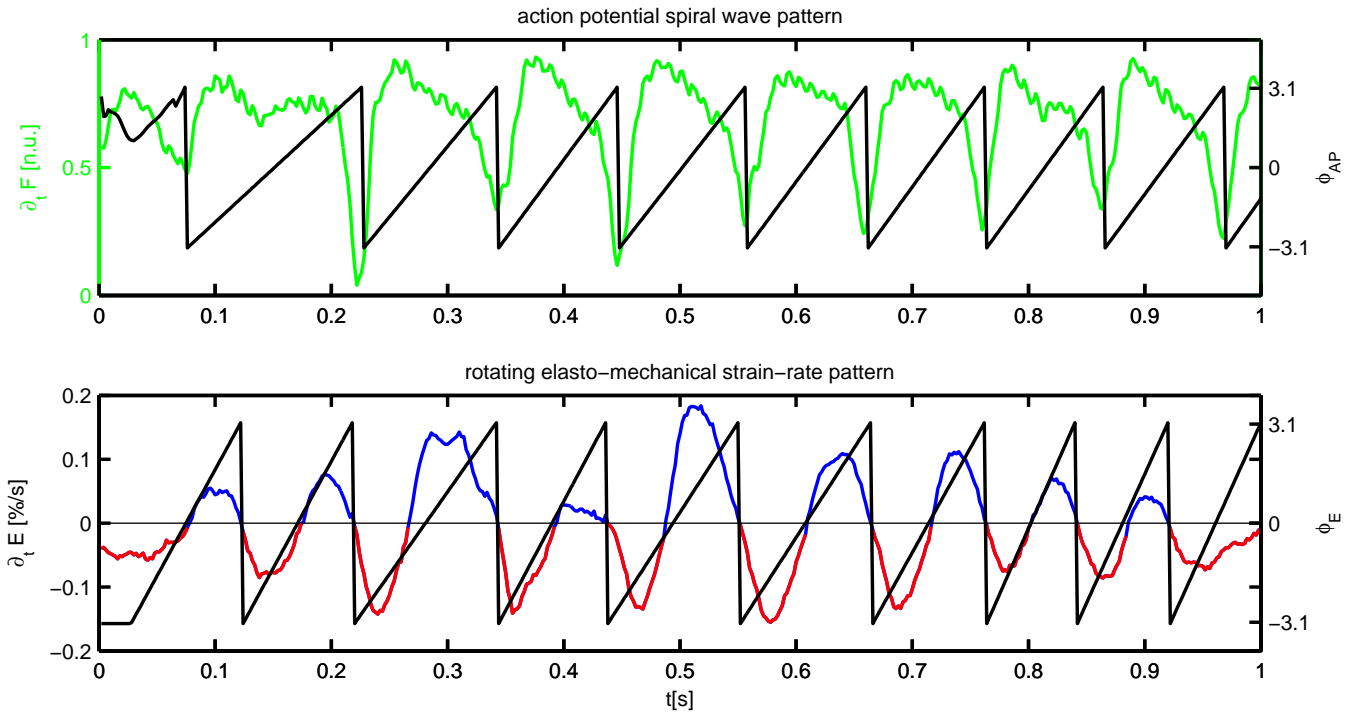

Figure 6.25: Reconstruction of phase representations $\phi_{A P}$ and $\phi_{\hat{E}}$, see below, of the electrical and mechanical patterns. Conversion of periodically fluctuationg signals into continuously increasing phase states of electrical or mechanical activation.

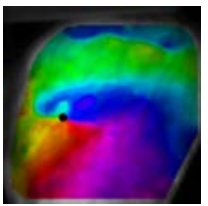

$324 m s$

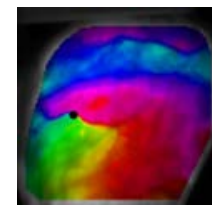

$350 m s$

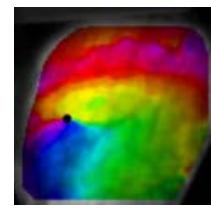

$386 m s$

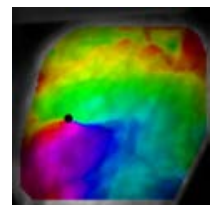

$410 \mathrm{~ms}$

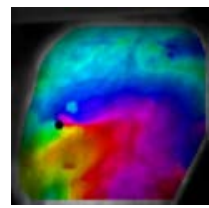

$446 m s$

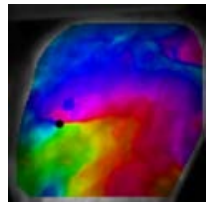

$460 m s$

Figure 6.26: Phase representation $\phi_{A P}$ of counter clock-wise rotating action potential spiral wave, shown in figure 6.22, on left ventricular surface of rabbit heart during ventricular fibrillation. Color code indicates phase state of electrical activation $\left(\phi\left(V_{m}\right)\right)$. Phase singularity (black dot) at center of field of view indicating spiral wave core.

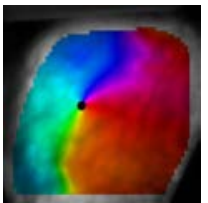

$244 \mathrm{~ms}$

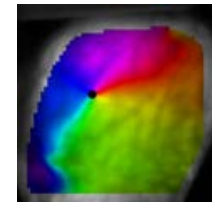

$264 m s$

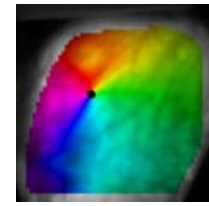

$292 m s$

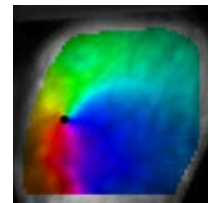

$322 m s$

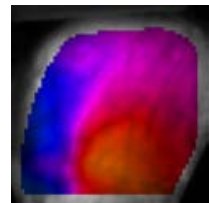

$370 m s$

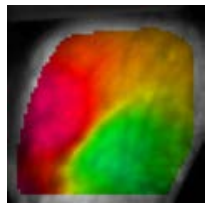

$400 \mathrm{~ms}$

Figure 6.27: Phase representation $\phi_{\hat{E}}$ of counter clock-wise rotating elasto-mechanical rate of deformation pattern on left ventricular surface of rabbit heart during ventricular fibrillation. Color code indicates phase state of mechanical activation. Phase singularity at center of field of view indicating rotational center of mechanical activity. 

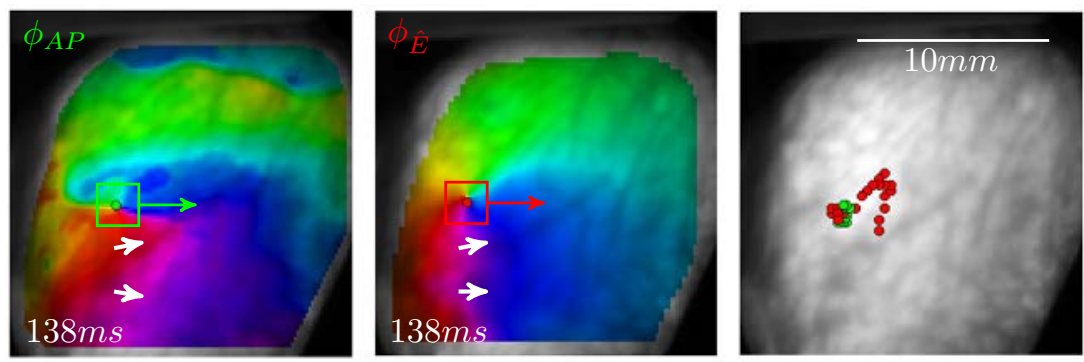

Figure 6.28: Co-localization of spiral wave core with rotational center of elastic deformation pattern on ventricular surface of the heart: electrical spiral wave core (green) at $\hat{\vec{x}}_{P S}^{A P}=(35.7,70.6) \pm(1.2,2.1)$ and elastic topological defect of rotational deformation pattern at $\hat{\vec{x}}_{P S}^{\hat{E}}=(44.2,63.7) \pm(7.4,5.9)$ during one rotation of the spiral wave rotor $(208 \mathrm{~ms}-322 \mathrm{~ms})$.

phase singularity being located at $\hat{\vec{x}}_{P S}^{A P}=(35.7,70.6) \pm(1.2,2.1)$ and the average postion of the mechanical phase singularity being located at $\hat{\vec{x}}_{P S} \hat{E}=(44.2,63.7) \pm(7.4,5.9)$ measured in pixels. Hence, both phase singularities were separated by an average distance of $d=10.9 \pm 7.3$ pixels within one rotation of the spiral wave, which meanwhile spanned across the entire imaged surface with dimensions larger than $\sim 100 \times 100$ pixels.

\section{Electromechanical Spiral Wave}

The recording suggests that the rotating elasto-mechanical rate of deformation pattern is the mechanical fingerprint of the electrical action potential spiral wave pattern. The two in-phase rotating electrical and mechanical patterns appear to belong to the same coupled electromechanical scroll wave rotor, with the electrical activity inducing accordingly similar elastic deformation patterns, as postulated in chapter 5 . The findings support the hypothesis that spiraling electrical activity in the heart leads to a spiral wave-like deformation of the heart muscle.

\subsubsection{Electromechanical Scroll Wave Activity inside Ventricular Wall}

In the previous section, coupled electromechanical spiral wave activity was observed on the surface of the heart. The following data set demonstrates that the same obervations of simultaneously appearing, rotational electrical and mechanical patterns during ventricular tachycardia and fibrillation, can also be made beneath the surface, when imaging the heart using ultrasound.

Figure 6.29 shows the electrocardiogram of an episode of wash-out fibrillation in a rabbit heart with an intermittent episode of polymorphic ventricular tachycardia during which large rotors appear in front of the camera. The heart resets to sinus wave activity at about $1.5 \mathrm{~s}$ after the start of the recording. The fibrillatory activity was induced, as described in the previous section, by a series of pacing stimuli applied to the epicardial surface of the heart. The electrical activity on the surface of the heart was filmed in multi-parametric imaging mode, using voltage-sensitive dye (Di-4-ANBDQPQ) as well as calcium-sensitive dye (Rhod2-AM). Still frames of the two raw videos are shown in figure 6.10. The post-processing of the fluorescence recordings was performed as described in section 6.4. The mechanical activity within the ventricular wall was filmed using ultrasound within a cross-section positioned as indicated in figures 6.18-6.19. The ultrasound transducer is visible in the top part of the images in the following image sequences. The mechanical activity on the surface of the heart was 


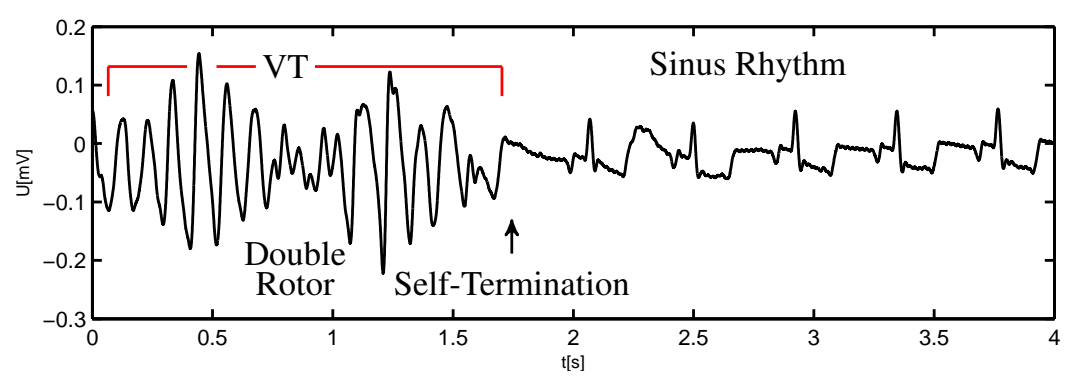

Figure 6.29: Electrocardiogram of self-terminating ventricular fibrillation $(t<0.0 \mathrm{~s})$ with intermittent polymorphic ventricular tachycardia (VT: $0.0 s \leq t \leq 2.0 s$ ) before transition to sinus rhythm $(t>2.0 \mathrm{~s})$ in rabbit heart. Episode was imaged using multi-modal, multi-parametric fluorescence and ultrasound imaging. Particularly during polymorphic ventricular tachycardia, the episode is determined by large spiral wave rotor activity, with one double rotor visible at $\sim 0.9 \mathrm{~s}$.

tracked within the fluorescence videos.

Figures 6.30 and 6.31 show the electrical activity on the ventricular surface of the rabbit heart during this episode. During the entire recording, both action potential and calcium transient wave patterns were found to be closely coupled and to exhibit the same patterns without any visible degenerations of the coupling, see figure 6.32. The calcium wave pattern was found to have the better image quality and was used for visualization. The image series show the calcium transient wave pattern (green) together with its phase representation (continuous colorcode), which was derived as described in the previous sections, with the phase-mapped fluorescent signal $\phi(x, y, t)_{C a}$ being a phase state angle $\phi \in[-\pi, \pi]$. Red color corresponds to the trespassing of the upstroke of a calcium transient wave front. The recording begins with a large clock-wise rotating spiral wave rotor, which occupies the entire left ventricle and performs about 4-5 rotations with rotational periods of $T \sim 120 \mathrm{~ms}$, see figure 6.30. In the beginning, the rotor presents a spiral wave core, indicated by the phase singularity in the phase representation (lower panel) that remains stationary in the lower right corner of the video for more than one complete rotation. Next, the phase singularity disappears and the excitation appears from now on to come from the subsurface to the surface. However, the rotational sense and angular speed remains the same and overall, the rotor seems to keep its clock-wise rotational sense until about $\sim 0.5 s$ of the recording. At $0.7 s$ a double rotor breaks through the surface and produces a figure-of-eight pattern, composed of two counter-rotating spiral waves, with the left spiral wave rotating in clock-wise and the right spiral wave rotating in counter-clock-wise directions respectively, see figure 6.31. The double rotor performs 2 rotations until the pairwise annihilation of the two spiral cores at $t=1044 \mathrm{~ms}$. The following pattern seems to follow a counter-clock-wise rotating pattern, around a rotational center that is not visible on the surface and seems to lay within the heart walls towards the base of the heart. The arrhythmic activity ends shortly thereafter, after 3-4 more rotations of the counter-clock-wise rotor. The three phases of the electrical pattern, consisting of one counterclock-wise rotating rotor, a double rotor and a clock-wise rotating rotor, can also be identified in the electrocardiogram. The electrocardiogram exhibits a torsade de pointes signature, see section 1.3, with the typical undulations of its amplitude. The node at $0.8-0.9 \mathrm{~s}$ corresponds to the period in which the fluorescence recordings show the double rotor, see figure 6.29. It is reasonable to assume that the imaged episode corresponds to polymorphic tachycardia rather than ventricular fibrillation, with an underlying wave pattern consisting of one or two scroll waves. ${ }^{44}$ 
Chapter 6. Intramural Scroll Wave Imaging during Ventricular Tachycardia and Fibrillation

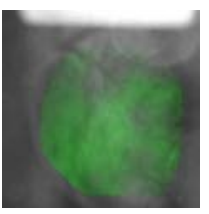

$128 m s$

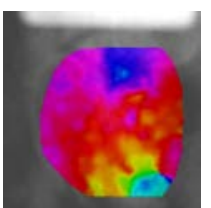

$128 m s$

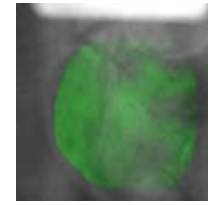

$144 m s$

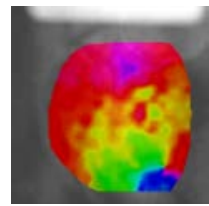

$144 m s$

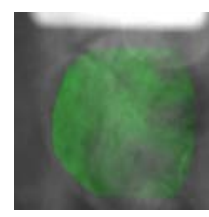

$156 m s$

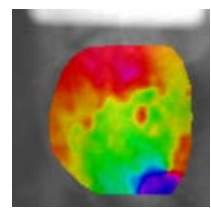

$156 \mathrm{~ms}$

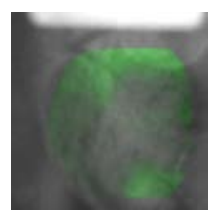

$184 m s$

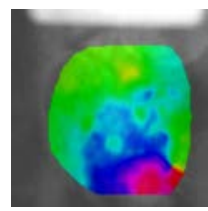

$184 m s$

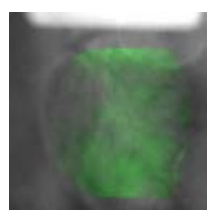

$212 m s$

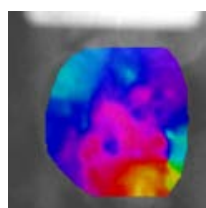

$212 m s$

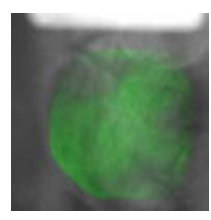

$240 \mathrm{~ms}$

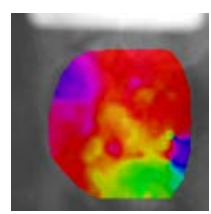

$240 \mathrm{~ms}$

Figure 6.30: Clock-wise rotating electrical spiral wave rotor (green) on left ventricular surface of rabbit heart during wash-out fibrillation and polymorphic ventricular tachycardia, measured with fluorescence imaging using calcium-sensitive dye (Rhod2-AM). Lower panel: phase representation $\phi$.

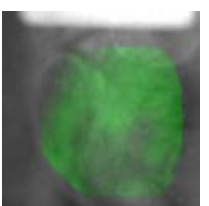

$672 m s$

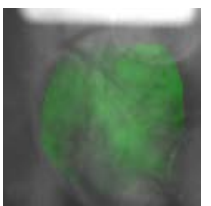

$820 \mathrm{~ms}$

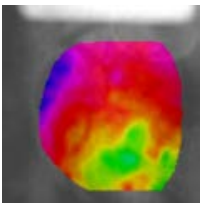

$672 m s$

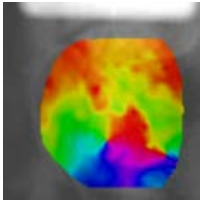

$820 m s$

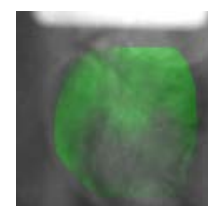

$692 m s$

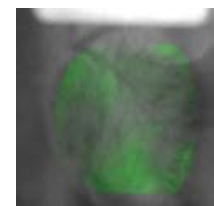

$848 m s$

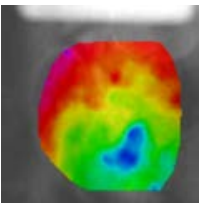

$692 m s$

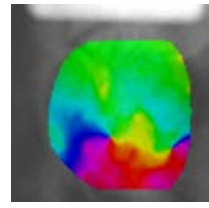

$848 \mathrm{~ms}$

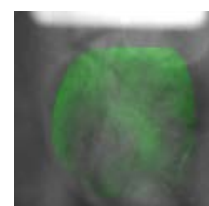

$712 \mathrm{~ms}$

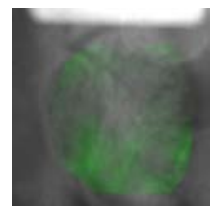

$872 \mathrm{~ms}$

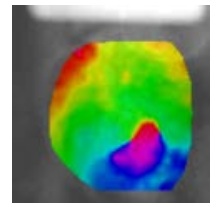

$712 \mathrm{~ms}$

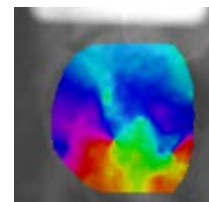

$872 m s$

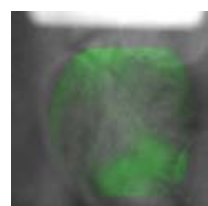

$732 \mathrm{~ms}$

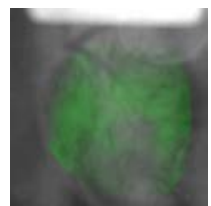

$900 \mathrm{~ms}$

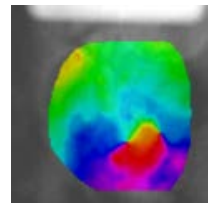

$732 \mathrm{~ms}$

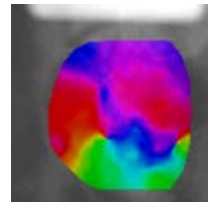

$900 \mathrm{~ms}$

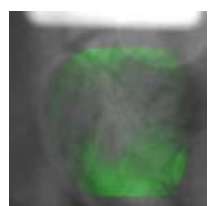

$748 \mathrm{~ms}$

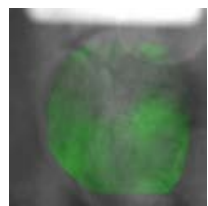

$992 \mathrm{~ms}$

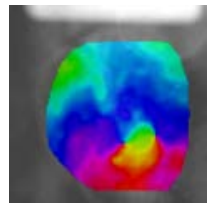

$748 \mathrm{~ms}$

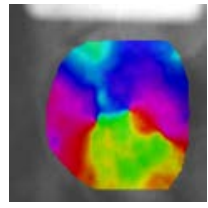

$992 \mathrm{~ms}$

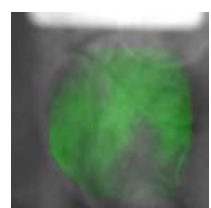

$792 \mathrm{~ms}$

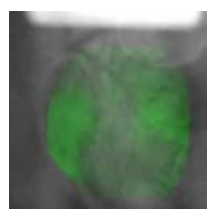

$1008 \mathrm{~ms}$

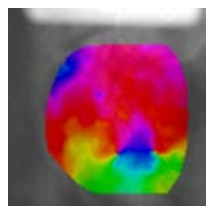

$792 m s$

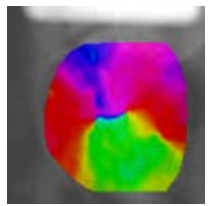

$1008 \mathrm{~ms}$

Figure 6.31: Double rotor composed of a pair of spiral waves producing a figure-of-eight pattern of electrical activity on the left ventricular surface of rabbit heart during wash-out fibrillation and polymorphic ventricular tachycardia, measured with fluorescence imaging using calcium-sensitive dye (Rhod2-AM). 

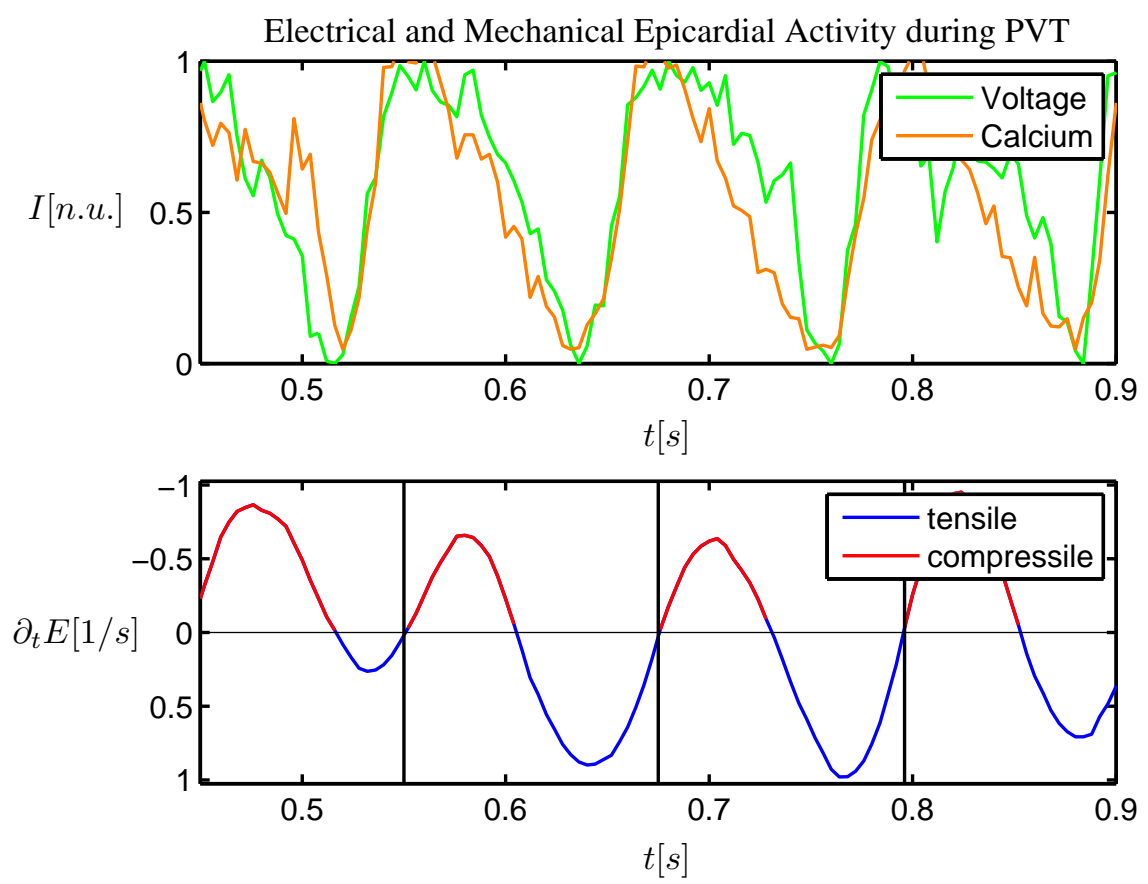

Figure 6.32: Electromechanical coupling during cardiac tachyarrhythmias: closely correlated electrical and mechanical activity on ventricular surface of rabbit heart during polymorphic tachycardia, see figure 6.29 for comparison with according electrocardiogram. Congruent action potential (green) and calcium (orange) spiral wave patterns at rotational periods of $\sim 120 \mathrm{~ms}$ and consequential alternating dilating (blue) and contracting (red) rates of deformation of the tissue (strain-rate), here shown for one examplary point on the tissue surface. Compare with figure 5.1 in chapter 5 .

\section{Coupling of Transmembrane Voltage $V_{m}$, Intracellular Calcium and Rate of Deformation dur- ing Ventricular Fibrillation}

Equally as described in the previous section, the mechanical activity on the ventricular surface was found to be closely related to the electrical activity, see figures 6.32, 6.33 and 6.34. Also, as described above, the action potential wave pattern was found to be congruent with the calcium transient wave pattern. Figure 6.32 shows a series of action potentials (green) and calcium transients (black) obtained from one site, located on the right side, vertically centered on the ventricle, together with the respective rate of deformation (strain-rate) of the local piece of tissue. All three curves are in agreement with the picture that was postulated and demonstrated in the simulations in chapter 5, with the upstrokes of the action potentials and calcium transients coinciding and each cycle of dilating (blue) and contracting (red) rates of deformation following the electrical activity, compare also with figures 1.6 and 5.1. In particular, the experimental data demonstrates that the coupled electromechanical activity continues to be composed of three subsequent waves during cardiac tachyarrhytmias, with the calcium cycling and intracelluar calcium release and onset of contraction following the transmembrane depolarization, at least for the type of arrhythmic activity that is presented here, with large rotors and spiral rotation periods in the order of $T \sim 100 \mathrm{~ms}$. 
Chapter 6. Intramural Scroll Wave Imaging during Ventricular Tachycardia and Fibrillation

\section{Rotational Epicardial Deformation Patterns}

The elasto-mechanical deformation pattern of the epicardial surface exhibited, similarly as described in the previous section, rotating patterns that matched the electrical activity. Figure 6.33 shows spiral wave-like rotating rate of deformation patterns on the ventricular surface, which were tracked within the fluorescence recordings shown in figures 6.30-6.31. The motion was tracked as described previously. Again, the frequencies of the cycles of dilating and contracting rates of deformation, the rotational sense as well as the angular speeds of the pattern match the electrical pattern's behavior, see also figure 6.36. From the beginning, the mechanical activity exhibits clock-wise rotating patterns that follow the rotations of the electrical spiral wave rotor until the electrical double spiral wave rotor appears. Here, the clock-wise rotating pattern persists, presumably as the resolution and sensitivity is not capable of resolving the smaller-scaled activity of the two rotors. However, towards the end of the recording the pattern starts to rotate, as the electrical pattern, in opposite direction. The phase representation of the mechanical pattern $\phi_{\hat{E}}$, see figure 6.34, exhibits phase singularities, which indicate accordingly the rotational centers and topological defects of the elastic pattern. The mechanical phase singularities appear to be approximately co-localized in time and space with the phase singularities featured by the electrical pattern. In the beginning of the recording, one can observe a clock-wise rotating pattern with a phase singular point in the lower half of the field of view. This phase singularity matches the electrical phase singularity that was found in the beginning of the recording in the lower right corner of the electrical pattern. However, the mechanical phase singularitiy periodically leaves and enters the field of view, while the overall pattern continues to rotate. During the presence of the double rotor, the mechanical pattern intermittently exhibits two phase singularities (for instance $t=748 \mathrm{~ms}$ ), which is not depictable from the strain-rate pattern directly shown in figure 6.33. Generally, it seems to be a feature of the elastic pattern, that its topological defects exhibit a higher mobility and deviate around its electrical counterparts.

\section{Intramural Spiral Wave-Like Rotating Deformation Patterns}

Simultaneously to the measurements performed on the surface, the intramural deformation was captured using ultrasound. The ultrasound transducer can be seen in the figures 6.30-6.34 in the top of each image. The imaging configuration is shown in figures 6.18 and 6.19. Figure 6.35 shows the elasto-mechanical rate of deformation pattern and its according phase representation. Generally, the patterns appear to be rotating, and, similarly as observed on the surface, retain a spiral wavelike character. Though imaged several millimeters beneath the optically imaged surface of the heart, the frequencies of the cycles of dilating and contracting rates of deformation, the rotational sense as well as the angular speeds match both the electrical and mechanical patterns on the surface of the heart, see also figure 6.36. In particular, from about $150 \mathrm{~ms}$ to about $420 \mathrm{~ms}$ it is possible to observe a clock-wise rotational pattern in all four acquired videos, that are the video showing the action potential spiral wave, the calcium transient spiral wave, the spiral wave-like rotating mechanical activity on the surface and the spiral wave-like rotating mechanical activity inside the ventricular wall. From $150 \mathrm{~ms}$ to about $300 \mathrm{~ms}$ both the epicardial action potential and calcium wave patterns exhibit a phase singularity that depicts the location of the core region of the electrical spiral wave on the surface, while from about $200 \mathrm{~ms}$ to $310 \mathrm{~ms}$ the epicardial elasto-mechanical activity exhibits a phase singularity that depicts the rotational center of the contractile motion and elastic deformation of the muscle on its surface, and lastly from $290 \mathrm{~ms}$ to about $420 \mathrm{~ms}$ the intramural elasto-mechanical activity as well exhibits a phase singularity that depicts a rotational center of the contractile motion and elastic deformation inside the muscle. 
Chapter 6. Intramural Scroll Wave Imaging during Ventricular Tachycardia and Fibrillation

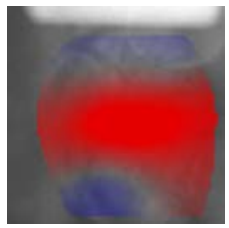

$244 m s$

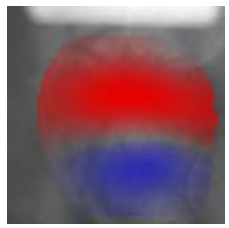

$568 m s$

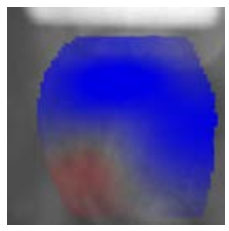

$652 m s$

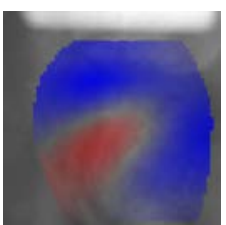

$780 m s$

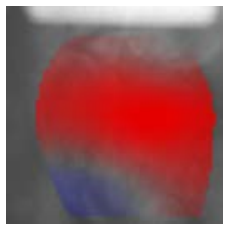

$260 \mathrm{~ms}$

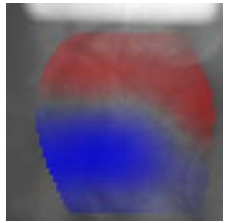

$596 m s$

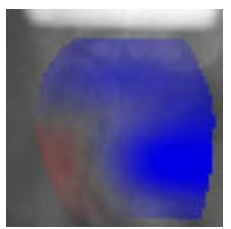

$668 m s$

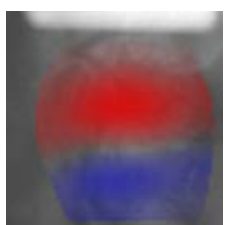

$800 \mathrm{~ms}$

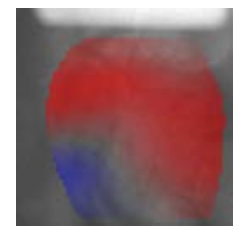

$272 m s$

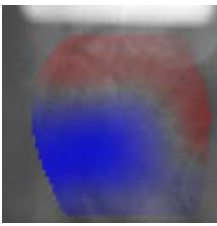

$600 \mathrm{~ms}$

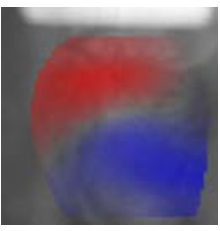

$692 m s$

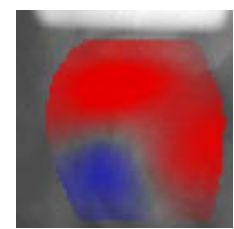

$824 \mathrm{~ms}$

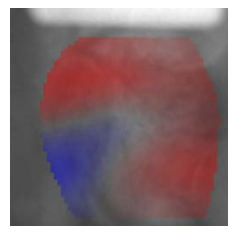

$280 \mathrm{~ms}$

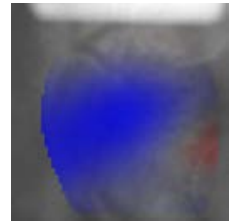

$608 \mathrm{~ms}$

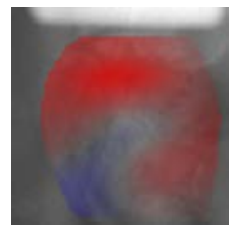

$708 \mathrm{~ms}$

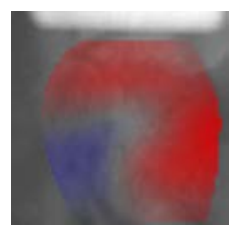

$844 m s$

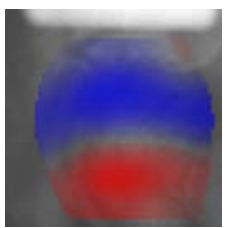

$304 m s$

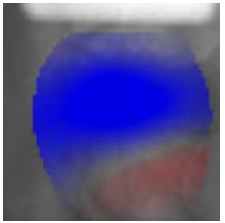

$620 \mathrm{~ms}$

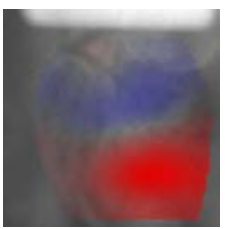

$732 \mathrm{~ms}$

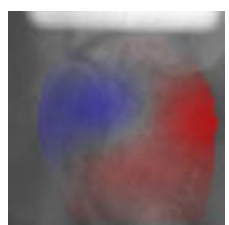

$856 \mathrm{~ms}$

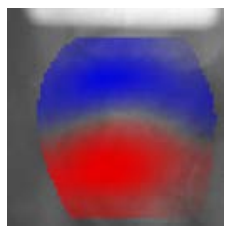

$320 \mathrm{~ms}$

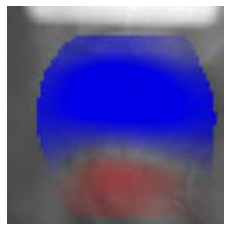

$636 \mathrm{~ms}$

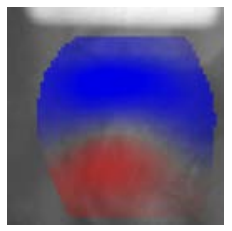

$756 \mathrm{~ms}$

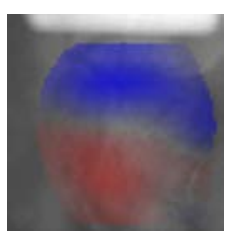

$896 \mathrm{~ms}$

Figure 6.33: Clock-wise spiral wave-like rotating elasto-mechanical rate of deformation pattern with dilating and contracting rates of deformation of tracked ventricular surface of rabbit heart. Rotational sense and angular speed matching the two electrical patterns shown in figures 6.30 and 6.31 . 
Chapter 6. Intramural Scroll Wave Imaging during Ventricular Tachycardia and Fibrillation

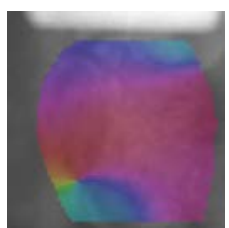

$244 m s$

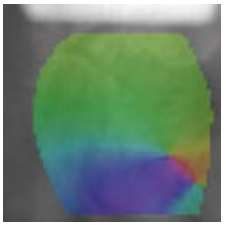

$608 m s$

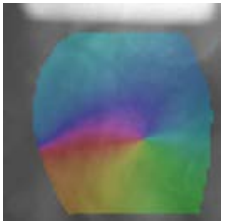

$768 m s$

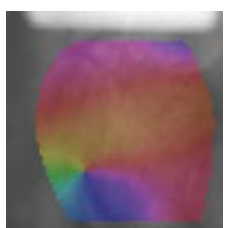

$260 \mathrm{~ms}$

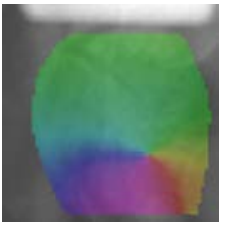

$620 m s$

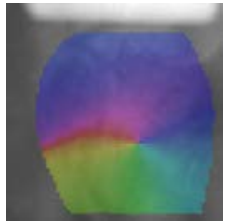

$786 m s$

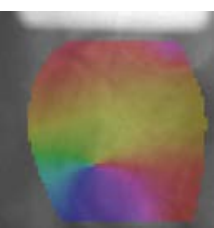

$272 m s$

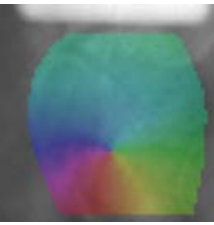

$640 \mathrm{~ms}$

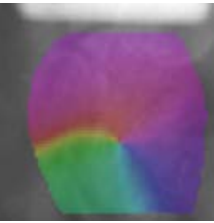

$804 m s$

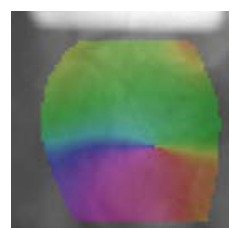

$296 m s$

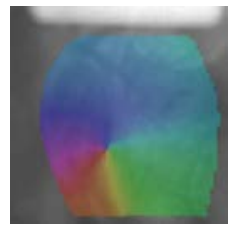

$656 m s$

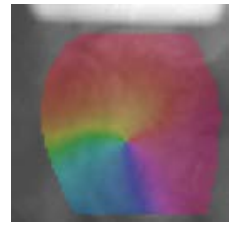

$824 m s$

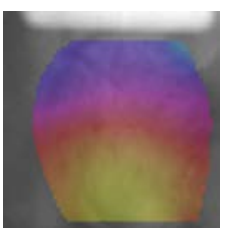

$340 \mathrm{~ms}$

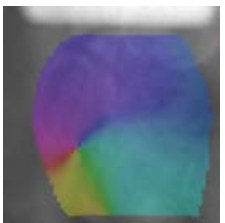

$672 m s$

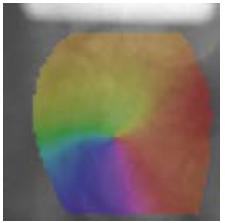

$840 \mathrm{~ms}$

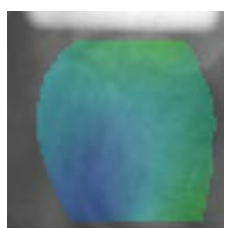

$412 m s$

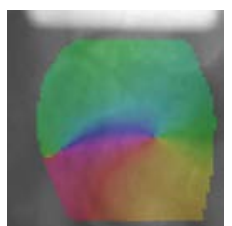

$748 m s$

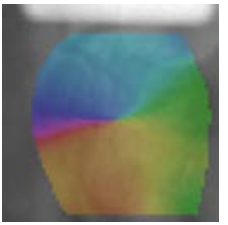

$888 \mathrm{~ms}$

Figure 6.34: Phase representation of clock-wise rotating elasto-mechanical rate of deformation pattern of tracked mechanical deformation of ventricular surface of rabbit heart. Rotational sense and angular speed matching the two electrical patterns shown in figures 6.30 and 6.31 .

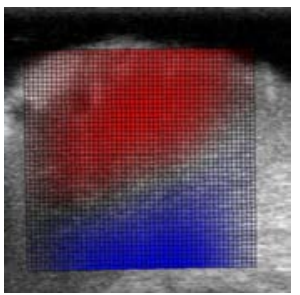

$339 m s$

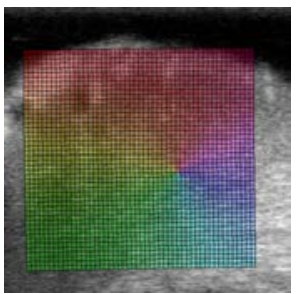

$339 m s$

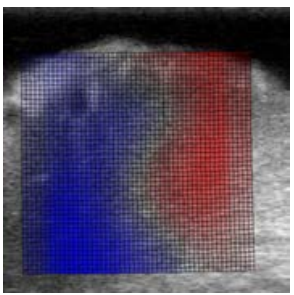

$372 m s$

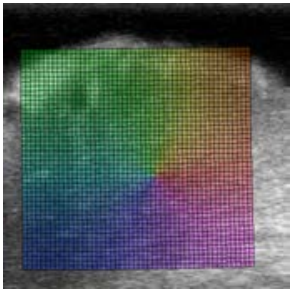

$372 m s$

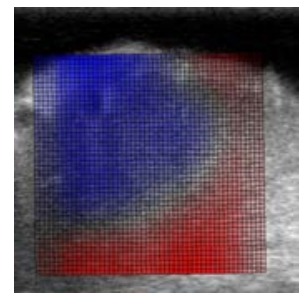

$394 m s$

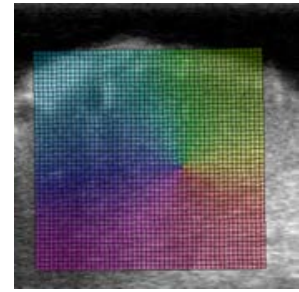

$394 m s$

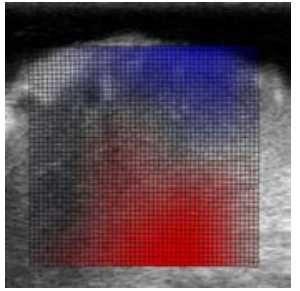

$424 m s$

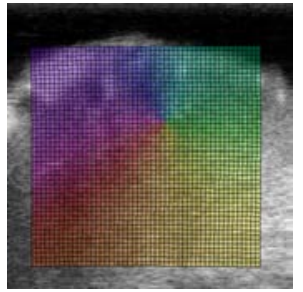

$424 m s$

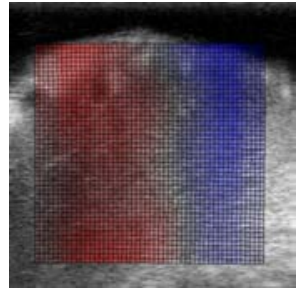

$440 \mathrm{~ms}$

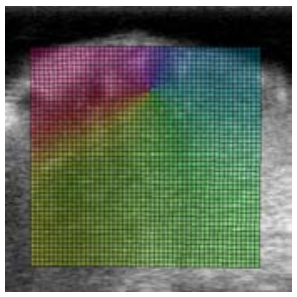

$440 \mathrm{~ms}$

Figure 6.35: Clock-wise rotating spiral wave-like mechanical deformation pattern at midwall of left ventricular wall of rabbit heart as imaged with ultrasound: phase singularity appearing during rotational deformation pattern of ventricular muscle, which was simultaneously imaged on the surface, see figure 6.34, and shortly after corresponding phase singularities appeared in the electrical and mechanical patterns on the surface $(120 \mathrm{~ms}-340 \mathrm{~ms})$. Spatial dimensions of image: $10.08 \mathrm{~mm} \times 10.0 \mathrm{~mm}$ 
Chapter 6. Intramural Scroll Wave Imaging during Ventricular Tachycardia and Fibrillation

Figure 6.36 shows the frequency spectra of the epicardial action potential and calcium transient spiral wave patterns, the epicardial and intramural rotating deformation patterns and the electrocardiogram. All spectra feature a pronounced peak at around $9.0 \mathrm{~Hz}\left(f_{A P}=8.8 \mathrm{~Hz} \pm 1.1 \mathrm{~Hz}, f_{C a}=\right.$ $8.8 \mathrm{~Hz} \pm 1.1 \mathrm{~Hz}, f_{\hat{E}}^{e p i .}=9.8 \mathrm{~Hz} \pm 1.1 \mathrm{~Hz}, f_{\hat{E}}^{\text {intram. }}=9.3 \mathrm{~Hz} \pm 1.1 \mathrm{~Hz}, f_{E C G}=8.7 \mathrm{~Hz} \pm 1.1 \mathrm{~Hz}$ ). However, only the spectra of the electrical patterns as well as of the electrocardiogram appear to be purely monochromatic. The spectra of the mechanical patterns feature additional peaks and fre-
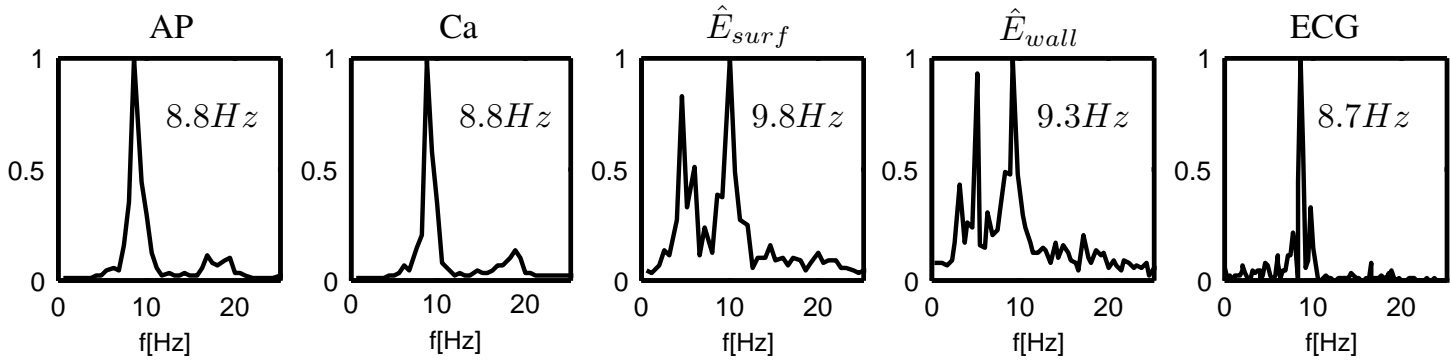

Figure 6.36: Frequency spectra of epicardial action potential spiral wave pattern $\left(f_{A P}=8.8 H z\right)$, epicardial calcium transient spiral wave pattern $\left(f_{C a}=8.8 \mathrm{~Hz}\right)$, epicardial elasto-mechanical rate of deformation pattern $\left(f_{\hat{E}}^{e p i .}=9.8 \mathrm{~Hz}\right)$, intramural elasto-mechanical rate of deformation pattern $\left(f_{\hat{E}}^{\text {intram. }}=9.3 \mathrm{~Hz} \pm 1.0 \mathrm{~Hz}\right)$ and electrocardiogram $\left(f_{E C G}=8.7 \mathrm{~Hz} \pm 1.1 \mathrm{~Hz}\right)$. All spectra show that the respective quantities seem to belong to the same underlying dynamical process.

quency contents at lower frequencies $(<8 \mathrm{~Hz})$. The nature of these lower frequency contents remained unclear. They may generally be a feature of the elastic wave dynamics and reflect passive elastic effects, correponding to relaxational motion of the tissue, that retributes stresses and strains within the actively contracting and deforming tissue. They may also partially be a result of the wave pattern reconstruction technique, which misses some of the rotational elastic activity and consequently produces larger inter-wave periods as it leaves some of the wave activity undetected. In conclusion, the frequency spectra indicate that all five quantities, which were measured independently from each other, belong to the same underlying dynamical process. The frequency spectra of the two-dimensional patterns were computed as the cumulative power spectra for each of the individual pixels of the patterns. Note, that the sampling duration of the analyzed time-series is very short for the computation of the power spectra. Their resolution is accordingly low. However, due to the monochromaticity of the dynamics the resultant $\pm 1 \mathrm{~Hz}$ uncertainty was found to be acceptable.

\section{Electromechanical Scroll Wave Rotor within Ventricular Wall}

The recording suggests that the rotating patterns, which are visible in the electrical as well as mechanical activity on the surface as well as inside the wall, are caused by coupled electromechanical scroll wave activity. In the first $0.5 \mathrm{~s}$ of the episode presented above, it can be assumed that the electrical activity is a clock-wise rotating scroll wave rotor, aligned in the ventricular wall as indicated in figure 6.37, producing a clock wise rotating spiral wave pattern on the epicardial surface as observed in figures 6.30 and 6.31 . The electrical vortex filament spans accordingly radially through the wall, touching the epi- and presumably also the endocardial wall, its tip constituting the spiral wave core on the surface. Accordingly, the spiral wave-like deformation of the cardiac muscle produces a mechanical topological defect line, as seen in figures 5.23 and 5.24 in chapter 5, that is aligned 


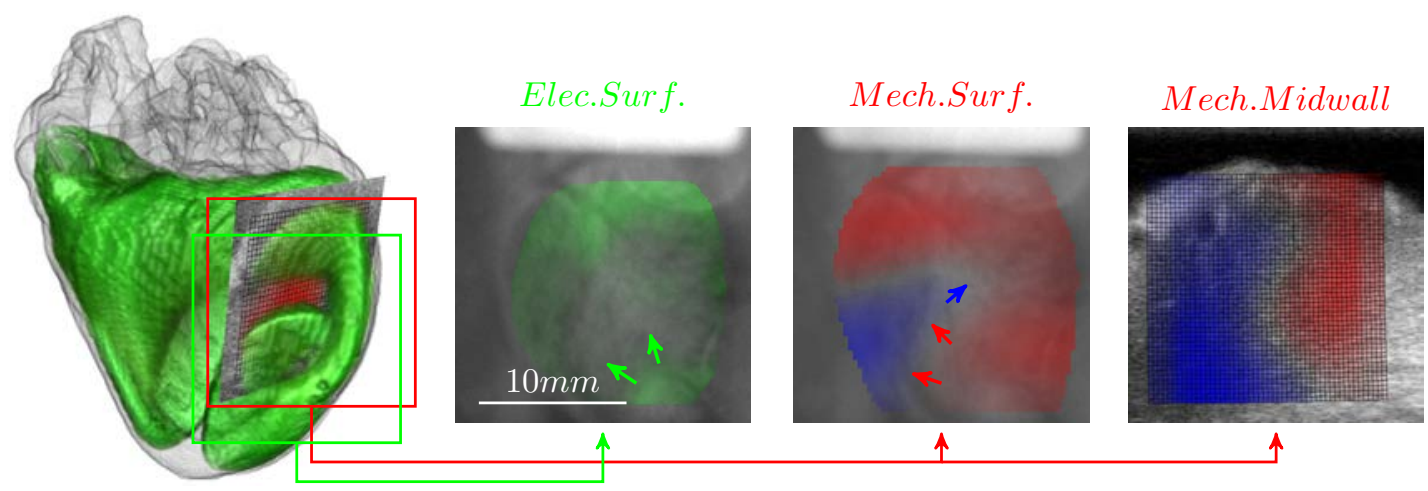

Figure 6.37: Electromechanical scroll wave rotor in the heart: action potential scroll wave rotor (green, simulation) inside ventricles of rabbit heart, causing spiral wave-like elastic deformations of the cardiac wall (dilating and contracting rates of deformation respectively). Electrical filament spans from epicardial to endocardial wall and spiral wave core on the surface is visible tip of the vortex filament touching the surface. Accordingly, rotational deformation produces mechanical topological defect line, see figures 5.23 and 5.24 in chapter 5, that is co-localized with electrical filament and intersects the ultrasound imaging plane at midwall, producing the phase singularity shown in figure 6.35 .

similarly as the electrical vortex filament inside the wall and intersects the ultrasound imaging plane. Here, the rotational deformation pattern is also visible within the cross-section and the intersection of the mechanical filament with the cross-section produces the phase singularity seen in figure 6.35 . Hence, the visualization of the intramural phase singularity may have provided a visualization of the rotational core of a scroll wave rotor inside the ventricular wall. 
Chapter 6. Intramural Scroll Wave Imaging during Ventricular Tachycardia and Fibrillation 
Chapter 7. Discussion

\section{Chapter 7}

\section{Discussion}

The work presented in this thesis explored a novel imaging technique for the visualization of the chaotic fibrillatory wave activity underlying cardiac arrhythmias. As outlined in the introduction, the technique aimed to employ the excitation-contraction coupling mechanism in the heart, a mechanism which maintains the coupling between the electrophysiological and contractile activity of the cardiac muscle. The main idea in this work was to inversely link contractile activity and mechanical deformation to electrical activity and to use information about the mechanical deformation of the heart to estimate the electrical activity pattern that lied inaccessibly within the cardiac muscle and that had initially caused the heart to deform. The problem was approached from different perspectives including imaging experiments with living cardiac tissue, development of computational techniques that allowed analysis of the experiments and development and use of computer simulations of cardiac tissue that allowed the investigation of the basic behavior of the fibrillating, contracting heart. This chapter briefly summarizes the results, compares them to related studies reported in the literature and evaluates their quality and significance. Lastly, the chapter provides an outlook that suggests future research.

\subsection{Summary of Results}

In this work, the fibrillatory wave activity that underlies ventricular tachycardia and fibrillation was imaged inside the heart muscle. High-speed fluorescence and ultrasound imaging were used simultaneously to capture the electrical as well as the mechanical activity of the heart on its surface and inside the heart muscle, see chapter 6. Experiments were conducted in intact, isolated Langendorffperfused rabbit hearts, in a setup that allowed to induce and control cardiac arrhythmias during imaging. While during cardiac tachyarrhymias, action potential and calcium spiral wave patterns were observed on the surface of the heart, it was found that the accordingly deforming cardiac muscle exhibited spiral wave-like rotating mechanical deformation patterns on the surface as well as inside the heart walls, see sections 6.6 .1 and 6.6.2 in chapter 6. The rotating elasto-mechanical patterns were found to share the same rotational directions and the same rotational periods and angular speeds respectively as the electrical patterns on the surface. In addition, the rotational centers of the elastomechanical patterns were found to be co-localized with the core region of the electrical spiral wave on the ventricular surface, see section 6.6.1. Intermittently, even the entire patterns were found to be congruent with the electrical spiral wave patterns. Both the dominant frequencies of the electrical and mechanical patterns were found to be identical with the dominant frequencies of the electrocardiogram during the fibrillatory activity, suggesting that both patterns were reflections of the same 
underlying activity and dynamics. The deformations of the cardiac muscle were tracked employing computer vision techniques, see sections 5.5.1, 6.4.3 and 6.5.3. The optical fluorescence recordings provided movies of the deforming ventricular surface, while the ultrasound speckle image recordings provided movies of the deforming inner ventricular wall. In addition to capturing the deforming tissue configurations, tracking enabled the collection and extraction of the fluorescent signal from the moving cardiac tissue, in the co-moving frame and in material coordinates of the deforming continuum body. Multi-parametric fluorescence imaging enabled to map action potential wave patterns as well as intracellular calcium concentrations on the surface of the heart simultaneously. It was found that the direct coupling between action potential waves and intracellular calcium release was maintained during the imaged fibrillatory episodes, such that the resulting imaged spatial-temporal patterns consisted of two closely coupled and congruent electrical patterns. Together with the above described behavior of the mechanical activity, the electromechanical activity as a whole remained closely coupled during fibrillation. The electromechanical activity showed to be composed of three subsequent immediately coupld waves, see also figure 5.1, with the action potential being immediately followed by intracellular calcium release and the calcium release being followed by a wave-like onset of contraction, such that on the tissue level excitation-contraction coupling manifested in two closely related spatial-temporal electrical and mechanical patterns. Moreover, as the imaging speeds were found to be sufficient to resolve the rotational elasto-mechanical patterns and allowed to follow the propagation of onset of contraction and wave-like spread of mechanical activation throughout the tissue, it was demonstrated that electromechanical waves in the heart appear to be composed of spreading action potential waves immediately followed by a wave-like spreading of deformation.

In chapter 5, a fundamental understanding of the structure of coupled electromechanical wave activity in the heart was developed. Using computer simulations, coupled electromechanical wave patterns, such as spiral and scroll waves, were studied in-silico. The computational modeling assumed homogenous active tension development and conversion of excitation into myofibril contraction along a local muscle fiber orientation, considering the highly complex, rotational anisotropic muscle fiber organization in myocardial tissue. Consequently, it was demonstrated that electrical activation patterns caused highly similar elasto-mechanical patterns during the fibrillatory contractions and deformations of the tissue, considering the highly anisotropic muscle fiber organization of cardiac muscle tissue. For instance, and in analogy to the experiments, electrical spiral wave patterns were found to induce spiral wave-like elastic deformation patterns, that similarly exhibited rotational centers as the electrical patterns. In two spatial dimensions, these rotational centers could be determined by computing phase singular points inside phase representation maps of the time-varying elasto-mechanical rate of deformation pattern. It was found that the phase singular points indicate elasto-mechanical topological defects within the dynamic elastic pattern, similarly as phase singular points derived from electrical spiral wave patterns indicate topological defects of that pattern, which correspond to the spiral wave tips or core regions. Here, the mechanical topological defects were determined to be co-localized with the core region of the electrical spiral wave pattern. In three spatial dimensions, scroll wave activity was found to induce a three-dimensional time-varying scroll wave-like rate of deformation pattern inside the deforming cardiac tissue volumes. The volumetric deformation patterns similarly developed rotational centers. Along these rotational centers, the spatial-temporal behavior of the deforming tissue resulted in the formation of filament-like elasto-mechanical topological defect lines, which were computed as lines of phase singularity correspondingly, emerging inside the three-dimensional, time-varying phase representations of the rate of deformation patterns, see section 5.4. The elasto-mechanical topological defect lines were found to retain a filament-like structure and 
to be co-localized with electrical scroll vortex wave filaments at the core region of the scroll wave rotor. Mechanical and electrical filaments appeared to be co-localized, often aligned in close proximity next to and wrapped around each other, sharing the same rotational direction or vorticity and angular speeds. Both lines of phase singularity, the ones derived from rotating electrical and the other ones from rotating mechanical activity, described similarly the scroll wave and its alignment and evolution over time. Hence, it was concluded that both electrical and mechanical filaments together constitute electromechanical scroll vortex wave filaments.

The findings obtained in the imaging experiments in the heart were complemented by experimental observations of rotating spiral wave-like elasto-mechanical deformation patterns in engineered cardiac cell culture preparations, see section 5.5.3. The cell culture preparations provided well-defined imaging conditions and exhibited spiraling mechanical activity within a two-dimensional plane, with one spiral wave rotor rotating around a stationary center of rotation at the center of the field of view. The activity was filmed and the induced two-dimensional deformations were captured using the same tracking techniques that were used to capture the deformations of the heart. The imaged situation came close to an idealized situation of an electromechanical spiral wave and was similarly simulated in the in-silico studies described above. The same analysis that was used to compute the phase singularities and to reveal the electrical and mechanical spiral wave cores in the computer simulations, also revealed the phase singularity and rotational center of the elasto-mechanical spiral wave pattern exhibited by the engineered cardiac cell culture preparation.

The computational framework, that allowed to study the interplay of coupled electromechanical wave activity, was developed within the scope of this work, see chapter 4. It allowed to simulate qualitatively electrical impulse propagation and spiral and scroll wave patterns in accordingly contracting and deforming cardiac tissue with underlying muscle fiber anisotropy. Later extensions, that were contributed by co-workers, see section 4.4 , lead to a simulation framework that allowed to simulate coupled electromechanical activity in realistically heart-shaped simulation domains. The heart geometries were obtained from the same rabbit hearts that were imaged in the experiments.

\subsection{Conclusion}

The findings obtained in the experiments and computer simulation studies together suggest that the rotating electrical and mechanical spiral wave or spiral wave-like activities correspond to the same coupled electromechanical spiral or scroll wave activity. Accordingly, in the rabbit heart, the spiraling electrical and rotating spiral wave-like mechanical patterns observed on the surface as well as within the cross-sectional imaging plane located at midwall, were the visible and in the experiments accessible parts of the underlying three-dimensional electromechanically coupled scroll wave activity, see also section 6.6.2. This implies that the imaging experiments performed in the rabbit heart provided visualizations of scroll wave activity within the ventricular wall during cardiac tachyarrhythmia and that these visualizations provide an experimental verification of the long posed hypothesis that scroll waves underlie ventricular tachycardia and fibrillation. ${ }^{48-50,59,65,103,107,228,229}$

The visualization of spiral or scroll waves inside the cardiac muscle during ventricular tachycardia or fibrillation was a long sought-after objective, ${ }^{103}$ that until now evaded experimental realization ${ }^{231}$ and has accordingly never been previously reported. The first experimental observation of a spiral wave in the heart was made in 1990 by Davidenko et al. ${ }^{31}$ imaging the surface of the heart using 
voltage-sensitive fluorescence imaging and observing the spiral wave accordingly on the epicardial surface. Since then, Pertsov et al. ${ }^{47}$ Baxter et al. ${ }^{75}$ Bernus et al.,${ }^{97}$ Wellner et al. ${ }^{138}$ Hillman et al. ${ }^{142}$ and Mitrea et al. ${ }^{183,204}$ aimed to extend this technique to visualize the electrical wave pattern within the cardiac muscle using optical techniques that employ combinations of light-tissue interactions similarly to fluorescence imaging and optical tomography, see also section 3.3.3 in chapter 3 . However, while it became possible to identify intramyocardial filaments in projections of scroll wave activity in video recordings of transilluminated ventricular pig heart muscle, ${ }^{183}$ it remains a major challenge to directly image rotor activity and vortex filament motion at midwall and to visualize the three-dimensional evolution of scroll wave patterns within the heart.

Electromechanical activity of the heart has never been imaged in the way as it was reported here, with the combined and simultaneous use of optical high-speed fluorescence imaging and high-speed ultrasound imaging techniques. The term electromechanical wave imaging was introduced in 2010 by Konofagou and co-workers. ${ }^{205,206,215}$ In their work, they demonstrated that the onset of contraction within the ventricular muscle, imaged at high-speeds using ultrasound, follows paths that are known to be conduction pathways of electrical activity. Rather than verifying the electrical conduction pathways simultaneously, they compared their results to results from other studies and reproductions of these results in computer simulations. Consequently, they provided visualizations of spread of mechanical activation throughout the heart and were able to distinguish activation patterns of cardiac arrhythmia, such as for instance atrio-ventricular dissociation, from periodic sinus activity. However, their use of the term electromechanical wave imaging refers to an experimental measuring technique that is solely based on ultrasound elastography imaging. In particular, they did not measure both electrical and mechanical activities together. The idea to use mechanical deformation of cardiac tissue to inversely identify the underlying action potential wave pattern intramurally was first proposed by Otani and co-workers. ${ }^{192}$ In their work, the possibility to use an inverse computational model with the mechanical deformation state of a piece of cardiac tissue and the underlying muscle fiber configuration as input to detect active stress distributions within myocardial tissue volumes was demonstrated in-silico. The technique was neither demonstrated to be able to provide visualizations of transient patterns, such as scroll waves, nor was it demonstrated to be used with real experimental data. The idea inspired this work, however, a different approach was followed as in work preceding this work ${ }^{199}$ it was found that electromechanical wave propagation in engineered cardiac tissue retains a wave-like character with a localizable wavefront and onset of contraction, similarly as described by Konofagou and co-workers. Here, it was aimed to reconstruct the isochronal wavefront structures directly from dynamic features of the elasto-mechanical activity.

Fluorescence imaging or optical mapping experiments with fully contracting cardiac tissue preparations, which were performed without the use of pharmacological uncoupling substances, were reported by Seo et al. ${ }^{193}$ and Bourgeois et al. ${ }^{198}$ and was presented in my own work preceeding this work. ${ }^{199}$ Post-acquisition removal of motion artifacts inside optical mapping recordings was reported by Rohde et al. ${ }^{122}$ and Svrcek et al. ${ }^{186}$ However, in the work of Rohde et al. ${ }^{122}$ the experiments were conducted including the use of uncoupling agents and the movies were registered allowing only translational motion and rotations. Here, the fluorescence imaging experiments had to be performed with fibrillating, strongly contracting and moving hearts and image registration was performed allowing nonlinear and large deformations. Similarly as in this work, Svrcek et al. ${ }^{186}$ aimed at describing the properties of motion artifacts. Seo et al. ${ }^{193}$ and Bourgeois et al. ${ }^{198}$ introduced markers to the tissue for motion tracking. Here, only the tissue texture was used for tracking. Fluorescence imaging of 
fibrillating, contracting hearts was never reported before.

In-silico studies of electromechanical wave activity in cardiac tissue were reported by Panfilov et al., ${ }^{106,148}$ Keldermann et al., ${ }^{144,181}$ Jarousse et al., ${ }^{164,201,214}$ Weise et al. ${ }^{207,208,233}$ and Cherubini et al. ${ }^{113,167,211}$ While the studies investigated the fundamental behavior of coupled electromechanical wave activity and revealed new phenomena that arise due to electromechanical feedback and found effects such as self-pacemaking activity, ${ }^{144}$ mechanically induced spiral wave break-up ${ }^{208}$ and changes of unpinning behavior of spiral waves, ${ }^{211}$ neither of the studies reported about the existence of topological defects arising in elasto-mechanical time-varying patterns and revealed the connection between the topologies of electrical scroll wave patterns and scroll wave-induced deformation patterns. The concept of electromechanical scroll vortex wave filaments relates to other studies that describe ventricular fribrillation in terms of scroll vortex wave filament dynamics. ${ }^{158}$

In summary, this work demonstrated that transient intramural wave activity in the heart can be imaged non-invasively using tomographic imaging such as ultrasound imaging. Generally, the here presented experimental approach provides new perspectives in biomedical research for the characterization of ventricular fibrillation. While it extends and complements the list of state-of-the-art imaging, such as fluorescence imaging, it can provide dynamical and statistical information about ventricular fibrillation similarily as it is known from optical techniques but within and throughout the heart muscle. This would allow to study interactions of vortex filaments with the heterogeneous cardiac substrate and provide the opportunity to image and assess directly the effectiveness of recent low-energy antifibrillation strategies, ${ }^{177,203}$ which aim to control the chaotic electrical wave pattern by recruiting anatomical structures as wave-emitting sites within the muscle, see the following outlook.

\subsection{Outlook}

The work presented in this thesis opens the path towards novel research investigating inverse electromechanical wave imaging in the heart and elucidating the fundamental mechanisms of the coupled electrical and mechanical activity and the heart muscle. Future research is required with regard to almost all aspects covered in this thesis. The work involved many different experimental and computational techniques, which as a methodology require further validation and enhancements but also with regard to their applicability to the scientific problem require further study. In general, this thesis explored the proof-of-concept of imaging the electromechanical wave activity in the heart. Future research should further extend the understanding of coupled electromechanical wave activity, using both experimental as well as computational methods and study the findings that were made in this thesis more systematically. Here, the suggestions for future research are ordered by their necessity and workability.

\subsubsection{Immediate}

In the experiments conducted in this work, it was demonstrated that the rotational center of one electrical rotor can be associated with the rotational center of its according elasto-mechanical deformation pattern. The simulations suggest that this finding also translates to situations composed of two or more rotors. In future studies, it should be investigated whether these findings can be repeated for two or multiple rotors, that is whether two or more rotors can be distinguished from each other and wether there are resolution limits. To validate the experiments, similar experiments that allow 
better control could be performed in engineered myocardial tissue preparations, see section 5.5.3 in chapter 5. Next, it should be investigated whether strobed or ECG-gated imaging allows the acquisition of pseudo three-dimensional contracting tissue volumes to perform the reconstruction analysis in three-dimensional time-varying volumes. For pacing or sinus activity, this should be relatively easy to realize. To image a scroll wave in strobed-imaging mode it consequently becomes necessary to establish protocols that allow to induce stationary scroll wave activity in the ventricles. To be able to perform the electromechanical wave pattern reconstruction in three dimensions, it becomes necessary to extend the elastic deformation tracking algorithms to be able to track three-dimensional deformations. The work may then also involve the creation of three-dimensional synthetic deforming volumetric speckle image data sets. Further study is also required in analyzing recordings that show different stages of ventricular fibrillation, or recordings that show polymorphic ventricular tachycardia compared to ventricular fibrillation, to establish whether it is possible to characterize the underlying activity based on the elasto-mechanical patterns using statistical or other dynamic properties. The experimental work should be accompanied and guided by numerical investigations.

The elastic deformation tracking technique presented in section 5.5.1 requires further enhancements and validation. Aside from determining its sensitivity and validating the accuracy of both the tracking and the regularization part using, for instance, synthetic data, the method could gain singificantly from integrating non-linear elastic behavior into the regularization procedure. Currently, the technique does not provide any means to estimate active stress. One immediate next step could be to add actively contracting elements into the elastic tracking procedure. This step would be the first move towards incorpoating an inverse computational technique similarly as described by Otani et al. ${ }^{192}$ into the technique.

Further validation and enhancements of the computational model are required. The in-silico studies should be repeated employing a computational model that is based on the finite element method in modeling the elasticity. Such an approach would increase the computational costst significantly, however, it would also provide more accurate material behavior and could be based on established constitutive behavior for cardiac tissue and models reported in the literature.

\subsubsection{Intermediate}

The imaging experiments should be repeated in larger animals, for instance pigs, and lastly also be performed in humans. As the imaging technique is, in principle, non-invasive, testing the technique in humans seems unproblematic. For instance, using transthoracic ultrasound imaging, the procedure would be entirely non-invasive. Possible candidates for imaging are patient with chronic atrial fibrillation and persistent ventricular tachycardia. It is imaginable that even persistent scroll wave activity could be imaged, using strobed B-mode or three-dimensional echocardiography.

Ultrasound imaging should be used in combination with panoramic fluorescence imaging and other techniques to capture the surface mechanics of the heart, ${ }^{216}$ capturing the electrical activity on the entire surface but also the deformations of the entire surface of the heart. Ideally, ultrasound imaging is going to be performed using novel phased-array ultrasound techniques, that provide real fourdimensional, that is time-varying three-dimensional recordings of the contracting cardiac muscle at high speeds. 


\subsubsection{Long-Term: Translation to Clinical Applications}

In the long term, the imaging technique presented in this thesis holds strong potential for diagnostic and therapeutic applications in the clinical setting. As it is compatible with wide-spread, non-invasive imaging technology such as ultrasound or magnetic resonance imaging, it is realistic to assume that similar imaging concepts will be adapted and incorporated in existing imaging technology in the future. For instance, using three-dimensional echocardiographic matrix-array ultrasound technology, monitoring of ablation procedures could benefit from live, three-dimensional visualizations of reentrant electromechanical activity to identify pro-arrythmic sites in the tissue for ablation. At the same time, focused ultrasound may provide the possibility to interact with the activity patterns, i.e. rotors and their cores and filaments, during the procedure. The imaging technique can be expected to become fully effective with the advent of high-speed four-dimensional imaging technology allowing the acquisition of large contracting myocardial tissue volumes, at high speeds and spatial resolutions sufficient to track electromechanical vortex filaments in a fully volumetric imaging mode throughout the cardiac muscle, for an in-depth and panoramic visualization of ventricular fibrillation. 
Chapter 7. Discussion 


\section{References}

[1] G. Mines; On circulating excitations in heart muscles and their possible relation to tachycardia and fibrillation; 4:43-52, Trans. R. Soc. Can., 1914

[2] W. Garrey; The nature of fibrillary contraction of the heart: its relation to tissue mass and form; 33:397-414, Am. J. Physiol., 1914

[3] T. Lewis; Oliver-Sharpey Lectures: On the nature of flutter and fibrillation of the auricle; 1:590593, 1:551-555, Br. Med. J., 1920-1921

[4] N. Wiener, A. Rosenblueth; The mathematical formulation of the problem of conduction of impulses in a network of connected excitable elements specifically in cardiac muscle; 16:205265, Arch. Inst. Cardiol. Mex., 1946

[5] M. Prinzmetal, E. Corday, I. C. Brill, A. L. Sellers, R. W. Oblath, W. A. Flieg; Mechanism of the auricular arrhythmias; 1:241-245, Circulation, 1950

[6] A. Hodgkin, A. Huxley; A quantitative description of membrane current and its application to conduction and excitation in nerve; Bulletin Math. Biology Vol. 52, No. 1/2, 1952

[7] A. Moscona, H. Moscona; The dissociation and aggregation of cells from organ rudiments of the early chick embryo; 86:287-301, J Anat., 1952

[8] R. FitzHugh; Impulses and physiological states in theoretical models of nerve membrane; 1, 445466, Biophys. J., 1961

[9] G. K. Moe; On the multiple wavelet hypothesis of atrial fibrillation; 140:183-188, Arch Int Pharmacodyn Ther., 1962

[10] J. Nagumo, S. Arimoto, S. Yoshizawa; An active pulse transmission line simulating nerve axon; 50:2061-2070, Proc IRE, 1962

[11] D. Noble; A modification of the Hodgkin-Huxley equations applicable to purkinje fibre action and pacemaker potentials; 160, 317-352, J. Physiol., 1962

[12] G. K. Moe, W. C. Rheinboldt, J. A. Abildskov; A computer model of atrial fibrillation; 67:200220 Am. Heart J., 1964

[13] V. Krinsky; Spread of excitation in an inhomogeneous medium (state similar to cardiac fibrillation); 11:676-683, Biophysics, 1966

[14] L. Malvern; Introduction to the Mechanics of a Continuous Medium; Prentice-Hall, 1969 
References

[15] A. Zaikin, A. Zhabotinsky; Concentration wave propagation in two-dimensional liquid-phase self-oscillating system; 225, 535-37, Nature, 1970

[16] J. Ambrose; Computerized transverse axial scanning (tomography): part 2. Clinical application; 46 102347, Br. J. Radiol., 1973

[17] M. Allessie, F. I. Bonke, F. J. Schopman, Circus movement in rabbit atrial muscle as a mechanism of trachycardia; 33:54-62, Circ. Res., 1973

[18] H. Davila, B. Salzberg, A. Waggoner; A large change in axon fluorescence that provides a promising method for measuring membrane potential; 241:159 160, Nat New Biol., 1973

[19] A. T. Winfree; Scroll-shaped waves of chemical activity in 3 dimensions; 181, 937, Science, 1973

[20] G. Salama, M. Morad, A. Waggoner; Merocyanine 540 as an optical probe of trans- membrane electrical activity in the heart; 191:485 487, Science, 1976

[21] B. Lucas, T. Kanade; An iterative image registration technique with an application to stereo vision; p. 674-679, International Joint Conference on Articial Intelligence, 1981

[22] N. A. Gorelova, J. Bures; Spiral waves of spreading depression in the isolated chicken retina; 14(5):353-63, J. Neurobiol., 1983

[23] B. J. Welsh, J. Gomatam, A. E. Burgess; Three-dimensional chemical waves in the BelousovZhabotinski reaction; 304, 611, Nature, 1983

[24] A. T. Winfree, S. H. Strogatz; Singular filaments organize chemical waves in three dimensions: IV. Wave taxonomy; Vol. 9, Issue 3, Physica D: Nonlinear Phenomena, 1983

[25] L. Cohen, S. Lesher; Optical monitoring of membrane potential: methods of multisite optical measurement; De Weer P, Salzberg BM, eds. New York: Wiley-Interscience, Optical Methods in Cell Physiology, 1986

[26] A. T. Winfree; When time breaks down; Princeton University Press, 1987

[27] W. Jahnke, C. Henze, A. T. Winfree; Chemical vortex dynamics in three-dimensional excitable media; 336, 662, Nature, 1988

[28] J. P. Keener; The dynamics of 3-dimensional scroll waves in excitable media; 31, 269, Physica D, 1988

[29] J. Roelandt, P. Serruys, N. Bom, W. Gussenhoven, C. Lancee and H. ten Ho; Intravascular real-time, two-dimensional echocardiography; Volume 4, Number 1, 63-67, DOI: 10.1007/BF01795127, The International Journal of Cardiac Imaging, 1989

[30] J. J. Tyson, K. A. Alexander, Y. Manoranjan, J. Murray; Spiral waves of cyclic AMP in a model of slime mold aggregation; 34, 193-207, Physica D, 1989

[31] J. M. Davidenko, P. F. Kent, D. R. Chialvo, D. C. Michaels, J. Jalife; Sustained vortexlike waves in normal isolated ventricular muscle; 87:8785-8789, Proceedings of the National Academy of Sciences, 1990 
References

[32] S. Jakubith, H. Rotermund, W. Engel, A. V. Oertzen, G. Ertl; Spatiotemporal concentration patterns in a surface reaction: Propagating and standing waves, rotating spirals, and turbulence; 65, 3013-6, Physical Review Letters, 1990

[33] O. Ramm, S. Smith; Real Time Volumetric Ultrasound Imaging System; SPIE Vol. 1231 Medical Imaging IV: Image Formation, 1990

[34] D. Barkley; A model for fast computer simulation of waves in excitable media; D 49, 61-70, Physica, 1991

[35] P. Grindrod; Patterns and Waves: Theory and Applications of Reaction-Diffusion Equations; Oxford Applied Mathematics and Computing Science, 1991

[36] J. Lechleiter, S. Girard, E. Peralta, D. Clapham; Spiral calcium wave propagation and annihilation in Xenopus laevis oocytes; 252:123-126, Science, 1991

[37] J. Ophir, I. Cspedes, H. Ponnekanti, Y. Yazdi, X. Li; Elastography: A quantitative method for imaging the elasticity of biological tissues; Ultrasonic Imaging, 1991

[38] P. A. Wolf, R. D. Abbott, W. B. Kannel; Atrial fibrillation as an independent risk factor for stroke: The Framingham study; 22, 983-8 Stroke, 1991

[39] A. Zhabotinsky; A history of chemical oscillations and waves; Chaos, 1991

[40] J.M. Davidenko, A.M. Pertsov, R. Salomonsz, W. Baxter, J. Jalife; Stationary and drifting spiral waves of excitation in isolated cardiac muscle; 355, 349, Nature, 1992

[41] E. Meron; Pattern Formation in Excitable Media; 218, 1, Phys. Rep., 1992

[42] M. C. Cross, P. C. Hohenberg; Pattern formation outside of equilibrium; 65, 851 Rev. Mod. Phys., 1993

[43] V. N. Biktashev, A. V. Holden, H. Zhang; Tension of organizing filaments of scroll waves; Vol. 347, No. 1685, Philosophical Transactions: Physical Sciences and Engineering, 1994

[44] R. A. Gray, J. Jalife, A. Panfilov, W. T. Baxter, C. Cabo, J. M. Davidenko, A. M. Pertsov; Nonstationary Vortexlike Reentrant Activity as a Mechanism of Polymorphic Ventricular Tachycardia in the Isolated Rabbit Heart; 91: 2454-2469 Circulation, 1994

[45] D. Johnston; Foundations of Cellular Neurophysiology; MIT Press, 1994

[46] A. Munuzuri, C. Innocenti, J. Flesselles, J. Gilli, K. Agladze, V. Krinsky; Elastic excitable medium; 50, 2 Physical Review, 1994

[47] A. Pertsov, W. Baxter, C. Cabo, R. Gray, J. Davidenko, J. Jalife; Transillumination of the Myocardial Wall allows Optical Recording of 3-dimensional Electrical Activity; 90:I-411, Circulation, 1994

[48] A. T. Winfree; Electrical turbulence in three-dimensional heart muscle; 266, 1003, Science, 1994 
References

[49] R. A. Gray, J. Jalife, A. V. Panfilov, W. T. Baxter, C. Cabo, J. M. Davidenko, A. M. Pertsov, A. V. Panfilov, P. Hogeweg, and A. T. Winfree; Mechanisms of cardiac fibrillation, 270, 1222, Science, 1995

[50] R. A. Gray, J. Jalife, A. Panfilov, W. T. Baxter, C. Cabo, J. M. Davidenko, A. M. Pertsov; Nonstationary Vortexlike Reentrant Activity as a Mechanism of Polymorphic Ventricular Tachycardia in the Isolated Rabbit Heart; 91: 2454-2469, Circulation, 1995

[51] R. Aliev, A. Panfilov; A simple two-variable model of cardiac excitation; 7:293-301, Chaos, Solitons, Fractals, 1996

[52] T. Amemiya, S. Kadar, P. Kettunen, K. Showalter; Spiral wave formation in three-dimensional excitable media; 77, 3244 Phys. Rev. Lett., 1996

[53] L. Gui, W. Merzkirch; A method of tracking ensembles of particle images; Experiments in Fluids, 465468 9, Springer, 1996

[54] R. Rohling, A. Gee, L. Berman; Three-dimensional Spatial Compounding of Ultrasound Images; volume 1, number 3, p177-193, Medical Image Analysis, 1996

[55] S. Gibson, B. Mirtich; A Survey of Deformable Modeling in Computer Graphics; Mitsubishi Electric Research Laboratory, 1997

[56] V. Hakim, A. Karma; Spiral Wave Meander in Excitable Media; The Large Core Limit; Vol. 97, No. 4, Phys. Rev. Lett., 1997

[57] P. Hunter, M. Nash, G. Sands; Computational electromechanics of the heart; pp. 345-408, (A. V. Panlov and A. V. Holden, eds.), Chichester: John Wiley \& Sons, Computational Biology of the Heart, 1997

[58] F. Fenton, A. Karma; Vortex dynamics in three-dimensional continuous myocardium with fiber rotation: filament instability and fibrillation; 8(1):20-47, Chaos, 1998

[59] R. A. Gray, A. Pertsov, J. Jalife; Spatial and temporal organization during cardiac fibrillation; Vol. 392, Nature, 1998

[60] J. Keener; Mathematical Physiology of the Heart; Springer, New York, 1998

[61] J. B. A. Maintz, M. A. Viergever; A survey of medical image registration; Vol. 2, No. 1, 1-36, Medical Image Analysis, 1998

[62] M. Nielsen; Finite Element Modeling in Surgery Simulation; 86(3), pp. 524-530, Proceedings of the IEEE: Special Issue on Virtual \& Augmented Reality in Medicine, 1998

[63] J. Schmitt; OCT elastography: Imaging microscopic deformation and strain in tissue; 3(6) 199211 Optical Express, 1998

[64] A. Verkhratsky, R. K. Orkand, H. Kettenmann; Glial calcium: homeostasis and signaling function; 78:99-141, Physiol. Rev., 1998

[65] F. X. Witkowski, L. J. Leon, P. A. Penkoske, W. R. Giles, M. L. Spano, W. L. Ditto, A. T. Winfree; Spatiotemporal evolution of ventricular fibrillation; Vol. 392, Nature, 1998 
References

[66] J. Schmitt; Optical Coherence Tomography (OCT): A Review; VOL. 5, NO. 4, IEEE Journal of Selected Topics in Quantum Electronics, 1999

[67] D. Bourguignon, M. Cani; Controlling Anisotropy in Mass-Spring Systems; iMAGIS, 2000

[68] A. Guyton, J. Hall; Textbook of Medical Physiology; Tenth Edition, Saunders, 2000

[69] H. Henry, V. Hakim; Linear stability of scroll waves; 85, 5328, Phys. Rev. Lett., 2000

[70] S. Periaswamy, J. Weaver, D. Healy, D. Rockmore, P. Kostelec, H. Farid; Differential Affine Motion Estimation for Medical Image Registration; 2000

[71] K. Rohr; Elastic Registration of Multimodal Medical Images: A Survey; 03/00, Künstliche Intelligenz, 2000

[72] P. Selskog, E. Brandt, L. Wigström, M. Karlsson; Visualization of myocardial strain-rate tensors from time-resolved 3D cine phase contrast MRI; 1870, Med. 9, Proc. Intl. Soc. Mag. Reson., 2001

[73] J. Walleczek; Self-Organized Biological Dynamics and Nonlinear Control; Cambridge University Press, 2000

[74] M. Zochowski, M. Wachowiak, C. Falk, L. Cohen, Y. Lam, S. Antic, D. Zecevic; Imaging Membrane Potential With Voltage-Sensitive Dyes; Concepts in Imaging and Microscopy, 198: 121, Biol. Bull., 2000

[75] W. Baxter, S. Mironov, A. Zaitsev, J. Jalife, A. Pertsov; Visualizing Excitation Waves inside Cardiac Muscle Using Transillumination; Volume 80, 516-530, Biophysical Journal, 2001

[76] B. Hille; Ion Channels of Excitable Membranes; Sinauer Associates Inc., Third Edition, 2001

[77] J. Humphrey; Cardiovascular Solid Mechanics, Cells, Tissue and Organs; Springer, 2001

[78] J. Jalife, O. Berenfeld, M. Mansour; Mother rotors and fibrillatory conduction: a mechanism of atrial fibrillation; Cardiovasc. Res. 54, 204-216, 2001

[79] P. Kohl, A. D. Nesbitt, P. J. Cooper, M. Lei; Sudden cardiac death by Commotio cordis: role of mechano-electric feedback; 50(2) 280-289, Cardiovascular Research, 2001

[80] S. H. Strogatz; Nonlinear Dynamics and Chaos; With Applications to Physics, Biology, Chemistry and Engineering; Westview Press, 2001

[81] B. Wünsche; The visualization of 3D stress and strain tensor elds; University of Auckland, 2001

[82] B. Bouma, G. Tearney; Handbook of optical coherence tomography; Marcel Dekker Inc., 2002

[83] D. M. Bers; Cardiac excitation-contraction coupling; 415(6868):198-205, Nature, 2002

[84] F. H. Fenton, E. M. Cherry, H. M. Hastings, S. J. Evans; Multiple mechanisms of spiral wave breakup in a model of cardiac electrical activity; 12, 852-892, Chaos, 2002

[85] H. Henry, V. Hakim; Scroll waves in isotropic excitable media: Linear instabilities, bifurcations, and restabilized states; 65, 046235, Phys. Rev. E, 2002 
References

[86] E. Konofagou, J. D’Hooge, J. Ophir; Myocardial Elastography - A Feasibility Study in Vivo; Vol. 28, No. 4, pp. 475 482, Ultrasound in Med. \& Biol. 2002

[87] C. Murry, M. Whitney, M. Laflamme, H. Reinecke, L. Field; Cellular therapies for myocardial infarct repair; 67:519 526; Cold Spring Harb Symp Quant; Biol., 2002;

[88] K. Pasumarthi, L. Field; Cardiomyocyte cell cycle regulation; 90, 1044-1054, Circ Res., 2002

[89] W. Zimmermann, M. Didie, G. Wasmeier, U. Nixdorff, A. Hess, I. Melnychenko, O. Boy, W. Neuhuber, M. Weyand, T. Eschenhagen; Cardiac Grafting of Engineered Heart Tissue in Syngenic Rats; 106, Circulation, 2002

[90] A. Duysak, J. J. Zhang, V. Ilankovan; Efficient modelling and simulation of soft tissue deformation using mass-spring systems; 1256, 337 342, International Congress Series, 2003

[91] J. Kybic, M. Unser; Fast Parametric Elastic Image Registration; IEEE Transactions on Image Processing, 2003

[92] W. Lauterborn, T. Kurz; Coherent Optics; Springer, 2003

[93] M. Lazebnik, D. Marks, S. Boppart; Functional optical coherence tomography for detecting neural activity through scattering changes; Vol. 28, No. 14, Optics Letters 2003

[94] S. Boppart; Optical Coherence Tomography: Technology and Applications for Neuroimaging; 529-541, 40 Psychophysiology, 2003

[95] J. Tsao, P. Boesiger, K. Pruessmann; k-t BLAST and k-t SENSE: Dynamic MRI with Frame Rate Exploiting Spatiotemporal Correlations; 50 1031-42, Magn. Reson. Med., 2003

[96] A. Benninghoff \& D. Drenckhahn; Anatomie, Band 2 (Elsevier GmbH, Urban \& Fischer Verlag, Munich, Germany, 2004), 16th edn.

[97] O. Bernus, M. Wellner, S. F. Mironov, A. M. Pertsov; Simulation of voltage-sensitive optical signals in three-dimensional slabs of cardiac tissue: application to transillumination and coaxial imaging methods; 50, 215-229, Phys. Med. Biol., 2004

[98] H. Dobrovolny, H. Elmariah, S. Kalb, J. Gilligan, J. Wikswo, D. Gauthier; Imaging Cardiac Dynamics Using Ultra-High-Power Light Emitting Diodes and Voltage Sensitive Dyes; 2004

[99] I. Efimov, V. Nikolski, G. Salama; Optical Imaging of the Heart; DOI: 10.1161/01.RES.0000130529.18016.35; 95;21-33, Circulation Research, 2004

[100] X. Huang, W. C. Troy, Q. Yang, H. Ma, C. R. Laing, S. J. Schiff, J. Wu; Spiral Waves in Disinhibited Mammalian Neocortex; 24(44):9897-9902, The Journal of Neuroscience., 2004

[101] K. Humphreys, J. Loughran, M. Gradziel, W. Lanigan, T. Ward, J. Murphy, C. O’Sullivan; Medical applications of Terahertz Imaging: a Review of Current Technology and Potential Applications in Biomedical Engineering, 2004

[102] M. Kovacs, J. Toth, C. Hetenyi, A. Malnasi-Csizmadia, J. Sellers; Mechanism of Blebbistatin Inhibition of Myosin II; DOI 10.1074/jbc.M405319200; Vol. 279, No. 34, Journal of Biological Chemistry, 2004 
References

[103] D. MacKenzie; Making Sense of a Heart Gone Wild; Vol. 303, Science, 2004

[104] R. A. Malkin, N. Kramer, B. Schnitz, M. Gopalakrishnan, A. L. Curry; Advances in electrical and mechanical cardiac mapping; Physiol. Meas. 26 (2005) R1R14, 2004

[105] J. Mosegaard; Realtime Cardiac Surgery Simulation; Diploma Thesis, Department of Computer Science, University of Aarhus, 2004

[106] M. Nash, A. Panfilov; Electromechanical model of excitable tissue to study reentrant cardiac arrhythmias; Prog. in Biophy. \& Mol. Biol. 85, 2004

[107] A. M. Pertsov; Scroll waves in three dimensions; 345-354, Cardiac Electrophysiology, from Cell to Bedside, 2004

[108] E. Pua, S. Idriss, P. Wolf, S. Smith; Real-time 3D Transesophageal Echocardiography; 26, 217- 232, Ultrasonic Imaging, 2004

[109] C. Ramanathan, R. Ghanem, P. Jia, K. Ryu, Y. Rudy; Noninvasive electrocardiographic imaging for cardiac electrophysiology and arrhythmia; Apr,10(4):422-8, Nat. Med., 2004

[110] M. Salomon, F. Heitz, G.-R. Perrin, J.-P. Armspach; A massively parallel approach to deformable matching of 3D medical images via stochastic differential equations; , 2004

[111] L. Taber; Nonlinear Theory of Elasticity, Applications in Biomechanics; World Scientific, 2004

[112] R. M. Zaritski, S. F. Mironov, and A. M. Pertsov; Intermittent self-organization of scroll wave turbulence in three-dimensional excitable media; 92, 168302, Phys. Rev. Lett., 2004

[113] D. Bini, C. Cherubini, S. Filippi; Viscoelastic Fitzhugh-Nagumo Models; E 72, 041929 PHYSICAL REVIEW, 2005

[114] P. Dayan, L. F. Abbot; Theoretical Neuroscience: Computational and Mathematical Modeling of Neural Systems; MIT Press, 2005

[115] E. Entcheva, H. Bien; Macroscopic optical mapping of excitation in cardiac cell networks with ultra-high spatiotemporal resolution; 92, 232-267, Progr. in Biophys. and Mol. Biol., 2005

[116] T. Eschenhagen, W. Zimmermann; Engineering Myocardial Tissue; 97, 1220-1231; Circulation Research 2005

[117] B. Gleich, J. Weizenecker; Tomographic Imaging using the Nonlinear Response of Magnetic Particles; 435, p. 1214-1217, Nature, 2005

[118] P. Helm, M. F. Beg, M. I. Miller, R. L. Winslow; Measuring and Mapping Cardiac Fiber and Laminar Architecture Using Diffusion Tensor MR Imaging; The Johns Hopkins University School of Medicine and Whiting School of Engineering, 2005

[119] J. Leor, Y. Amsalem, S. Cohen; Cells, scaffolds, and molecules for myocardial tissue engineering; 105, 151-163, Pharmacology \& Therapeutics, 2005

[120] A. Nealen, M. Müller, R. Keiser, E. Boxerman, M. Carlson; Physically Based Deformable Models in Computer Graphics; EUROGRAPHICS, The Eurographics Association, 2005 
References

[121] A. Panfilov, R. Keldermann, M. Nash; Self-Organized Pacemakers in a Coupled ReactionDiffusion-Mechanics System; Phys. Rev. Lett. 95, 258104, 2005

[122] G. Rohde, B. Dawant, S. Lin; Correction of Motion Artifact in Cardiac Optical Mapping Using Image Registration; IEEE Transactions on Biomedical Engineering, Vol. 52, No. 2, 2005

[123] R. F. Schmidt, F. Lang, G. Thews; Physiologie des Menschen (Springer Medizin Verlag, Heidelberg, Germany, 2005), 29th edn.

[124] M. Brezinski; Optical Coherence Tomography, Principles and Applications; Elsevier, 2006

[125] R.H. Clayton, E.A. Zhuchkov, A.V. Panfilov; Phase singularities and filaments: Simplifying complexity in computational models of ventricular fibrillation; 90, 378-398, Progress in Biophysics and Molecular Biology, 2006

[126] R. Huber, M. Wojtkowski, J. Fujimoto; Fourier Domain Mode Locking (FDML): A new laser operating regime and applications for optical coherence tomography; Vol. 14, Issue 8, pp. 32253237, doi:10.1364/OE.14.003225, Optics Express, 2006

[127] Kirckpatrick S. J., Wang R., Duncan D.; OCT-based elastography for large and small deformations; Vol. 14, No. 24, Optics Express, 2006

[128] M. W. Kay, J. M. Rogers; Epicardial rotors in panoramic optical maps of fibrillating swine ventricles; Proceedings of the 28th IEEE EMBS Annual International Conference, 2006

[129] M. W. Kay, G. P. Walcott, J. D. Gladden, S. B. Melnick, J. M. Rogers; Lifetimes of Epicardial Rotors in Panoramic Optical Maps of Fibrillating Swine Ventricles; 291(4): H1935-H1941H, Am. J. Physiol. Heart Circ. Physiol., 2006

[130] R. C. P. Kerckhoffs, S. N. Healy, T. P. Usyk, A. D. McCulloch; Computational Methods for Cardiac Electromechanics; Vol. 94, No. 4, Proceedings of the IEEE, 2006

[131] C. Mistretta; Highly constrained backprojection for time-resolved MRI; 55 30-40 , Magn. Reson. Med., 2006

[132] M. Mohr; A Hybrid Deformation Model of Ventricular Myocardium; Dissertation, Universität Fridericiana Karlsruhe, 2006

[133] J. Mosegaard; Cardiac Surgery Simulation, Graphics Hardware meets Congenital Heart Disease; Dissertation, Department of Computer Science, University of Aarhus, 2004

[134] R. FitzHugh, E. M. Izhikevich; FitzHugh-Nagumo Model; 1(9):1349., Scholarpedia, 2006

[135] T. Shimizu, H. Sekine, J. Yang, Y. Isoi, M. Yamato, A. Kikuchi, E. Kobayashi, T. Okano; Polysurgery of cell sheet grafts overcomes diffusion limits to produce thick, vascularized myocardial tissues; 10.1096/fj.05-4715fje, The FASEB Journal, 2006

[136] L. Tung, Y. Zhang; Optical imaging of arrhythmias in tissue culture; 39, S2-S6 Journal of Electrophysiology, 2006

[137] Y. Wang, Y. Rudy, Applications of the method of fundamental solutions to potential-based inverse electrocardiography, 34(8):1272-1288, Annals of Biomedical Engineering, 2006 
References

[138] M. Wellner, O. Bernus, S. Mironov, A. Pertsov; Multiplicative Optical Tomography of Cardiac Electrical Activity; 51, 4429-4446, Phys. Med. Biol., 2006

[139] L. Qi; Eigenvalues and invariants of tensors; 325, 1363-1377, J. Math. Anal. Appl., 2006

[140] T. Bansagi, O. Steinbock; Negative lament tension of scroll rings in an excitable system; 76, 045202, Phys. Rev. E, 2007

[141] V. Fedorov, I. Lozinsky, E. Sosunov, E. Anyukhovsky, M. Rosen, C. Balke, I. Efimov; Application of blebbistatin as an excitation-contraction uncoupler for electrophysiologic study of rat and rabbit hearts; Heart Rhythm, 4:619-626, 2007

[142] E. Hillman, O. Bernus, E. Pease, M. Bouchard, A. Pertsov; Depth-resolved optical imaging of transmural electrical propagation in perfused heart; Vol. 15, No. 26, Optics Express, 2007

[143] J. Hung, R. Lang, F. Flachskampf, S. K. Shernan, M. L. McCulloch, D. B. Adams, J. Thomas, M. Vannan, T. Ryan; 3D Echocardiography: A Review of the Current Status and Future Directions; 20:213-233, J. Am. Soc. Echocardiogr.; 2007

[144] R. Keldermann,M. Nash, A. Panlov; Pacemakers in a Reaction-Diffusion Mechanics System; DOI: 10.1007/s10955-006-9219-3, Vol. 128, Nos. 1/2, July, Journal of Statistical Physics, 2007

[145] A. Krupa, G. Fichtinger, G. Hager; Full Motion Tracking in Ultrasound Using Image Speckle Information and Visual Servoing; 2007

[146] J. W. Lin, L. Garber, Y. R. Qi, M. G. Chang, J. Cysyk, L. Tung; Region of slowed conduction acts as core for spiral wave reentry in cardiac cell monolayers; 294: H58-H65, Am J Physiol Heart Circ Physiol, 2007

[147] B. A. Lloyd, G. Szekely, M. Harders; Identification of Spring Parameters for Deformable Object Simulation; Vol. 13, No. 5, IEEE Transactions on Visualization and Computer Graphics, 2007

[148] A. V. Panfilov, R. H. Keldermann, M. P. Nash; Drift and breakup of spiral waves in reactiondiffusion-mechanics systems; No. 19, Vol. 104, Proceedings of the National Academy of Sciences, 2007

[149] B. Preim, D. Bartz; Visualization in Medicine - Theory, Algorithms and Applications; The Morgan Kaufmann Series in Computer Graphics, Elsevier, 2007

[150] A. Pumir,V. Nikolski, M. Hörning, A. Isomura, K. Agladze, K. Yoshikawa, R. Gilmour, E. Bodenschatz, V. Krinsky; Wave Emission from Heterogeneities Opens a Way to Controlling Chaos in the Heart; DOI: 10.1103/PhysRevLett.99.208101, Physical Review Letters, 2007

[151] M. Raffel, C. Willert, S. Wereley, J. Kompenhans; Particle Image Velocimetry, A Practical Guide; Springer, 2007

[152] E. Regar, P. Serruys, T. van Leeuwen; Optical Coherence Tomography in Cardiovascular Research; Taylor \& Francis 2007

[153] J. M. Rogers, G. P. Walcott, J. D. Gladden, S. B. Melnick, M. Kay; Panoramic Optical Mapping Reveals Continuous Epicardial Reentry during Ventricular Fibrillation in the Isolated Swine Heart; Vol. 92, 1090-1095, Biophysical Journal, 2007 
References

[154] L. Tung, J. Cysyk; Imaging fibrillation/defibrillation in a dish; 40, S62-S65, Journal of Electrophysiology, 2007

[155] C. Wachinger, W. Wein, N. Navab: Three-Dimensional Ultrasound Mosaicing; In: Ayache, N., Ourselin, S., Maeder, A. (eds.) MICCAI 2007, Part II. LNCS, vol. 4792, pp. 327-335. Springer, Heidelberg, 2007

[156] J. Whiteley, M. Bishop, D. Gavaghan; Soft Tissue Modelling of Cardiac Fibres for Use in Coupled Mechano-Electric Simulations; 69: 2199-2225, DOI 10.1007/s11538-007-9213-1, Bulletin of Mathematical Biology, 2007

[157] T. Bansagi, O. Steinbock; Three-dimensional spiral waves in an excitable reaction system: Initiation and dynamics of scroll rings and scroll ring pairs; 18, 026102, Chaos, 2008

[158] R. H. Clayton, Vortex filament dynamics in computational models of ventricular fibrillation in the heart; 18, 043127, Chaos, 2008

[159] Männer J., Thrane L., Norozi K., Yelbuz M.; High-Resolution In Vivo Imaging of the CrossSectional Deformations of Contracting Embryonic Heart Loops Using Optical Coherence Tomography; 237:953961, Developmental Dynamics 2008

[160] J. Balzer, H. Kühl, T. Rassaf, R. Hoffmann, P. Schauerte, M. Kelm, A. Franke; Real-time Transesophageal Three-dimensional Echocardiography for Guidance of Percutaneous Cardiac Interventions: First Experience; 97:565-574, DOI 10.1007/s00392-008-0676-3; Clin. Res. Cardiol., 2008

[161] D. M. Bers; Calcium cycling and signaling in cardiac myocytes; 70:2349, Annu. Rev. Physiol., 2008

[162] W. Drexler, J. Fujimoto; Optical Coherence Tomography: Technology and Applications (Biological and Medical Physics, Biomedical Engineering); Springer, 2008

[163] D. W. Evertson, M. R. Holcomb, M. D. C. Eames, M. Bray, V. Y. Sidorov, J. Xu, H. Wingard, H. M. Dobrovolny, M. C. Woods, D. J. Gauthier, J. P. Wikswo; High-Resolution High-Speed Panoramic Cardiac Imaging System; Vol. 55, No. 3, IEEE Transactions on Biomedical Engineering, 2008

[164] O. Jarousse, T. Fritz, O. Dössel; A modied Mass-Spring system for myocardial mechanics modeling; IFMBE Proceedings 22, pp. 19431946, Springer, 2008

[165] P. Bittihn, G. Luther, E. Bodenschatz, V. Krinsky, U. Parlitz, S. Luther; Far field pacing supersedes anti-tachycardia pacing in a generic model of excitable media; New Journal of Physics, 2008

[166] E. M. Cherry, F. H. Fenton; Visualization of spiral and scroll waves in simulated and experimental cardiac tissue; New Journal of Physics, 2008

[167] C. Cherubini, S. Filippi, P. Nardinocchi, L. Teresi; An electromechanical model of cardiac tissue: Constitutive issues and electrophysiological effects; 562-573, 97, Progress in Biophysics and Molecular Biology, 2008 
References

[168] J. M. Rogers, G. P. Walcott, J. D. Gladden, S. B. Melnick, R. E. Ideker, M. W. Kay; Epicardial wavefronts arise from widely distributed transient sources during ventricular fibrillation in the isolated swine heart; New Journal of Physics, 2008

[169] B. D. Soriano, M. Hoch, A. Ithuralde, T. Geva, A. J. Powell, B. D. Kussman, D. A. Graham, W. Tworetzky, G. R. Marx; Matrix-Array 3-Dimensional Echocardiographic Assessment of Volumes, Mass and Ejection Fraction in Young Pediatric Patients With a Functional Single Ventricle: A Comparison Study with Cardiac Magnetic Resonance; 117:1842-1848, Circulation, 2008

[170] P. Pathmanathan, J. Whiteley; A numerical method for cardiac mechano-electric simulations; OxMOS: New Frontiers in the Mathematics of Solids, University of Oxford, 2008

[171] L. J. Wang, E. A. Sobie; Mathematical model of the neonatal mouse ventricular action potential; American Journal of Physiology, Heart and Circulatory Physiology, Vol 294 no. 6, 2008

[172] P. Bittihn; Kontrolle von Spiralwellenaktivität in Erregbaren Medien; Diplom Thesis, Drittes Physikalisches Institut, Georg-August-University Göttingen, 2009

[173] P. Chowdhury, W. R. Lewis, R. A. Schweikert, J. E. Cummings; Ablation of atrial brillation: What can we tell our patients? 76, 543-50, Cleveland Clinic Journal of Medicine, 2009

[174] T. Terasawa; Comparative e?ectiveness of radiofrequency catheter ablation for atrial brillation; 151, 191-202, Annals of Internal Medicine, 2009

[175] M. Cross, H. Greenside; Pattern Formation and Dynamics in Nonequilibrium Systems, Cambridge University Press, 2009

[176] Multielectrode Mapping of the Heart; 5, 423-439, DOI: 10.1007/978-0-387-79403-717, Cardiac Bioelectric Therapy, 2009

[177] F. Fenton, S. Luther, E. Cherry, N. Otani, V. Krinsky, A. Pumir, E. Bodenschatz, R. Gilmour; Termination of atrial fibrillation using pulsed low-energy far-field stimulation; Circulation, 2009

[178] I. Grulkowski, M. Gora, M. Szkulmowski, I. Gorczynska, D. Szlag, S. Marcos, A. Kowalczyk, M. Wojtkowski; Anterior segment imaging with Spectral OCT system using a high-speed CMOS camera; Vol. 17, No. 6, Optics Express, 2009

[179] M. Gora, K. Karnowski, M. Szkulmowski, B. Kaluzny, R. Huber, M. Wojtkowski; Ultra highspeed swept source OCT imaging of the anterior segment of human eye at $200 \mathrm{kHz}$ with adjustable imaging range; Optics Express, 2009

[180] P. A. Iaizzo; Handbook of Cardiac Anatomy, Physiology and Devices; Second Edition, Springer, 2009

[181] R. Keldermann; Mechanisms of Ventricular Fibrillation - The role of mechano-electrical feedback and tissue heterogeneity; Dissertation, University of Utrecht, 2009

[182] M. Matchett et al.; The implantable cardioverter debrillator: Its history, current psychological impact and future; 6, 43-50, Expert Review of Medical Devices, 2009 
References

[183] B. G. Mitrea, M. Wellner, A. M. Pertsov, Monitoring intramyocardial reentry using alternating transillumination; 31 ${ }^{\text {st }}$ Annual International Conference of the IEEE EMBS, 2009

[184] M. Radisic, V. G. Fast, G. Vunjak-Novakovic; Optical Mapping of Impulse Propagation in Engineered Cardiac Tissue; Part A, Volume 15, Number 4, TISSUE ENGINEERING 2009

[185] L. Song, C. Kim, K. Maslov, L. Wang; High-speed dynamic 3D photoacoustic imaging of sentinel lymph node in a murine model using an ultrasound array; doi:10.1118/1.3168598; 36, 3724, Med. Phys., 2009

[186] M. Svrcek, S. Rutherford, A. Y. H. Chen, I. Provaznik, B. Smaill; Characteristics of Motion Artifacts in Cardiac Optical Mapping Studies; $31^{\text {st }}$ Annual International Conference of the IEEE EMBS, 2009

[187] J. Weizenecker, B. Gleich, J. Rahmer, H. Dahnke, J. Borgert; Three-Dimensional Real-Rime in vivo Magnetic Particle Imaging; 54, p.L1-L10, Physics in Medicine and Biology, 2009

[188] Y. Zhao et al.; Patch clamp technique: Review of the current state of the art and potential contributions from nanoengineering; 222, 1-11, Proceedings of the Institution of Mechanical Engineers, Part N: Journal of Nanoengineering and Nanosystems, 2009

[189] X. Huang, W. Xu, J. Liang, K. Takagaki, X. Gao, J. Wu; Spiral Wave Dynamics in Neocortex; Vol. 68, p978990, Neuron, 2010

[190] E. M. Izhikevich; Dynamical Systems in Neuroscience: The Geometry of Excitability and Bursting; MIT Press, 2010

[191] X. Jie, V. Gurev, N. Trayanova; Mechanisms of mechanically induced spontaneous arrhythmias in acute regional ischemia; 106: 185192. doi: 10.1161/circresaha.109.210864, Circ. Res., 2010

[192] N. Otani, S. Luther, R. Singh, R. F. Gilmour; Transmural Ultrasound-based Visualization of Patterns of Action Potential Wave Propagation in Cardiac Tissue; Annals of Biomedical Engineering, 2010

[193] K. Seo, M. Inagaki, S. Nishimura, I. Idaka, M. Sugimachi, T. Hisada, S. Sugiura; Structural Heterogeneity in the Ventricular Wall Plays a Significant Role in the Initiation of StretchInduced Arrhythmias in Perfused Rabbit Right Ventricular Tissues and Whole Heart Preparations; Circulation Research, 106:176-184, 2010

[194] R. Tung, N. G. Boyle, K. Shivkumar; Catheter ablation of ventricular tachycardia. 122, e38991, Circulation, 2010

[195] M. Uecker, S. Zhang, D.Voit, A. Karaus, K. Merboldt, J. Frahm: Real-time MRI at a Resolution of $20 \mathrm{~ms}$; 23, it NMR in Biomedicine, 2010

[196] S. Zhang, M. Uecker, D. Voit, K. Merboldt, J. Frahm; Real-time Cardiovascular Magnetic Resonance at High Temporal Resolution: Radial FLASH with Nonlinear Inverse Reconstruction; 12:39, Journal of Cardiovascular Magnetic Resonance, 2010

[197] R. M. Bell, M. M. Mocanu, D. M. Yellon, Retrograde heart perfusion: the Langendorff technique of isolated heart perfusion; Jun;50(6):940-50, J. Mol. Cell Cardiol., 2011 
References

[198] E. B. Bourgeois, A. D. Bachtel, J. Huang, G. P. Walcott, J. M. Rogers, Simultaneous optical mapping of transmembrane potential and wall motion in isolated, perfused whole hearts; Journal of Biomedical Optics, Vol. 16(9), 2011

[199] J. Christoph; Electromechanical Wave Propagation in Engineered Heart Tissue; Diplom Thesis, Georg-August University Göttingen, 2011

[200] J. Jalife; Deja vu in the theories of atrial brillation dynamics; 89, 766-775, Cardiovascular Reseacrh, 2011

[201] O. Jarousse; Modified Mass-Spring System for Physically Based Deformation Modeling; Dissertation, Karslruhe Institute of Technology, 2011

[202] P. Lee, C. Bollensdorff, T. A. Quinn, J. P. Wuskell, L. M. Loew, P. Kohl; Single-sensor system for spatially resolved, continuous, and multiparametric optical mapping of cardiac tissue; 8:14821491, Heart Rhythm, 2011

[203] S. Luther, F. H. Fenton, B. G. Kornreich, A. Squires, P. Bittihn, D. Hornung, M. Zabel, J. Flanders, A. Gladuli, L. Campoy, E. M Cherry, G. Luther, G. Hasenfuss, V. I Krisnky, A. Pumir, R. F. Gilmour, E. Bodenschatz; Low-energy control of electrical turbulence in the heart; Vol. 475, Nature, 2011

[204] B. G. Mitrea, B. J. Caldwell, A. M. Pertsov; Imaging electrical excitation inside the myocardial wall; Vol. 2, No. 3, Biomed. Opt. Express, 2011

[205] J. Provost, V. Thanh-Hieu Nguyen, D. Legrand, S. Okrasinski, A. Costet, A. Gambhir, H. Garan, E. E. Konofagou; Electromechanical wave imaging for arrhythmias; Physics in Medicine and Biology, 2011

[206] J. Provost, W.-N. Lee, K. Fujikura, E.E. Konafogou; Imaging the electromechanical activity of the heart; 108, 8565-8570, Proceedings of the National Academy of Sciences, 2011

[207] L. D. Weise, M. P. Nash, A. V. Panfilov; A discrete model to study reaction-diffusionmechanics systems; 6(7): e21934. doi: 10.1371/journal.pone.0021934, PLoS ONE, 2011

[208] L. D. Weise, A. V. Panfilov; New mechanism of spiral wave initiation in a reaction-diffusion mechanics system; 6(11): e27264. doi: 10.1371/journal.pone.0027264, PLoS ONE, 2011

[209] Global status report on noncommunicable diseases 2010. World Health Organization, Geneva, 2011

[210] Y. Yua, L. M. Santos, L. A. Mattiace, M. L. Costa, L. C. Ferreira, K. Benabou, A. H. Kime, J. Abrahams, M. V. L. Bennetth, R. Rozentala; Reentrant spiral waves of spreading depression cause macular degeneration in hypoglycemic chicken retina; Proceedings of the National Academy of Sciences, vol. 109, no. 7, 2011

[211] C. Cherubini, S. Filippi, A. Gizzi; Electroelastic unpinning of rotating vortices in biological excitable media; Physical Review E, 2012

[212] R. Deo, C. M. Albert; Sudden Cardiac Death, Epidemiology and Genetics of SUdden Cardiac Death; Circulation, 125:620-637, 2012 
References

[213] T. J. Herron, P. Lee, J. Jalife; Optical Imaging of Voltage and Calcium in Cardiac Cells and Tissues; Circ. Res., 110:609-623, 2012

[214] O. Jarousse; Modified Mass-Spring System for Physically Based Deformation Modeling; Dissertation, Karlsruher Institut fr Technologie, KIT Scientific Publishing, 2012

[215] E. E. Konofagou, J. Provost; Electromechanical wave imaging for noninvasive mapping of the 3D electrical activation sequence in canines and humans in vivo; 45, 856-864 Journal of Biomechanics, 2012

[216] J. I. Laughner, S. Zhang, H. Li, C. C. Shao, I. R. Emov; Mapping cardiac surface mechanics with structured light imaging; 303, H712-H720, American Journal of Physiology - Heart and Circulatory Physiology, 2012

[217] P. Lee, P. Yan, P. Ewart, P. Kohl, L. M. Loew, C. Bollensdorff ; Simultaneous measurement and modulation of multiple physiological parameters in the isolated heart using optical techniques; 464(4): 403414, NIH, 2012

[218] P. Lee, F. Taghavi, P. Yan, P. Ewart, E. A. Ashley, L. M. Loew, P. Kohl, C. Bollensdorff, C. E. Woods; InSitu Optical Mapping of Voltage and Calcium in the Heart; Volume 7, Issue 8, PLoS ONE, 2012

[219] J. Schweizer, M. Loose, M. Bonny, K. Kruse, I. Mönch, P. Schwille; Geometry sensing by self-organized protein patterns; Proceedings of the National Academy of Sciences, Vol. 109, No. 38,2012

[220] P. S. Spector, D. D. Correa de Sa, E. S. Tischler, N. C. Thompson, N. Habel, J. StinnettDonnelly, B. E. Benson, P. Bielau, J. H.T. Bates; Ablation of multi-wavelet re-entry: general principles and in silico analyses; 14, 106-111, Europace, 2012

[221] R. R. Tilz, A. Rillig, A. Thum, A. Arya, P. Wohlmuth, A. Metzner, S. Mathew, Y. Yoshiga, E. Wissner, K. Kuck, F. Ouyang; Catheter Ablation of Long-Standing Persistent Atrial Fibrillation; Heart Rhythm Disorders, Vol. 60, No, 19, 2012

[222] F. Vadakkumpadan, H. Arevalor, C. Ceritoglu, M. Miller, N. Trayanova; Image-Based Estimation of Ventricular Fiber Orientations for Personalized Modeling of Cardiac Electrophysiology; 31(5): 1051-1060, EEE Trans. Med. Imaging., 2012

[223] L. D. Weise, A. V. Panfilov; Emergence of spiral wave activity in a mechanically heterogeneous reaction-diffusion-mechanics system; 108: 228104. doi: 10.1103/physrevlett.108.228104, Phys Rev Lett, 2012

[224] O. V. Aslanidi, T. Nikolaidou, J. Zhao, B. H. Smaill, S. H. Gilbert, A. V. Holden, T. Lowe, P. J. Withers, R. S. Stephenson, J. C. Jarvis, J. C. Hancox, M. R. Boyett, H. Zhang; Application of micro-computed tomography with iodine staining to cardiac imaging, segmentation, and computational model development; 32(1):8-17, IEEE Trans. Med. Imaging., 2013

[225] P. Bittihn; Complex Structure and Dynamics of the Heart; Dissertation, Georg-AgustUniversity, Göttingen, 2013

[226] P. Dähmlow, S. Alonso, M. Bär, M. J. B. Hauser; Twists of Opposite Handedness on a Scroll Wave; 110, 234102, Physical Review Letters, 2013 
References

[227] D. Hornung; Cardiac Arrhythmia Termination on the Vascular and Organ Scale; Dissertation, Georg-August-University, Göttingen, 2013

[228] A. Karma; Physics of Cardiac Arrhythmogenesis; 4:313377, Annu. Rev. Condens. Matter Phys., 2013

[229] S. V. Pandit, J. Jalife; Rotors and the Dynamics of Cardiac Fibrillation, 112:849-862, Circulation, 2013

[230] D. Sato, D. M. Bers, Y. Shiferaw; Formation of Spatially Discordant Alternans Due to Fluctuations and Diffusion of Calcium; Volume 8, Issue 12, PLoS ONE, 2013

[231] B. H. Smaill, J. Zhao, M. L. Trew; Three-Dimensional Impulse Propagation in Myocardium; 112:834-848, Circulation Research, 2013

[232] S. Stein; Three-Dimensional Electromechanical Computer Model of the Heart Based on a Discrete Particle Simulation; Master Thesis, Georg-August-University, Göttingen, 2013

[233] L. D. Weise, A. V. Panfilov; A Discrete Electromechanical Model for Human Cardiac Tissue: Effects of Stretch-Activated Currents and Stretch Conditions on Restitution Properties and Spiral Wave Dynamics; DOI: 10.1371/journal.pone.0059317, PLoS ONE, 2013

[234] P. Bittihn; Complex Structure and Dynamics of the Heart; Springer, 2014

[235] G. Seiden, S. Curland; The tongue as an excitable medium; New Journal of Physics, 2015

[236] User: ZooFari. File: Heart diagram-en.svg URL http://commons.wikimedia.org/ wiki/File:Heart_diagram-en.svg Published under CC BY-SA 3.0: http://creativecommons.org/ licenses/by-sa/3.0/deed.en; modified image may be reused under the same conditions.

[237] User: Martanopue. File: Geographic tongue.JPG URL https://commons.wikimedia.org/wiki/File:Geographic_tongue_(cropped).jpg Published under CC BY-SA 3.0: http://creativecommons.org/ licenses/by-sa/3.0/deed.en; modified image may be reused under the same conditions.

[238] American Heart Association, http://www.heart.org/HEARTORG/Conditions/Arrhythmia/ AboutArrhythmia/, August 2014

[239] C. Mewis, R. Riessen, I. Spyridopoulos; Kardiologie compact, Alles für Station und Facharztprüfung; Georg Thieme Verlag, 2. unveränderte Auflage, 2006

[240] M. E. Josephson, Clinical Cardiac Electrophysiology, Techniques and Interpretations; Wolters Kluwer, Lippincott Williams \& Wilkins, Fourth Edition, 2008

[241] American Heart Association, http://www.heart.org/HEARTORG/Conditions/Arrhythmia/PreventionTreatmentofArrhythmia/, August 2014

[242] Fuji / Visualsonics Vevo 2100 Imaging System, Operator Manual Revision: 1.1; Visualsonics Inc., Toronto, Canada 


\section{References}




\section{List of Figures}

1.1 Two-dimensional Electromechanical Spiral Wave Patterns . . . . . . . . . . . . . 13

1.2 Spirals in Nature . . . . . . . . . . . . . . . . . . . . . . . . . 14

1.3 Vortex Filaments . . . . . . . . . . . . . . . . . . . . 16

1.4 Vortex Filaments . . . . . . . . . . . . . . . . . . . . . . 16

1.5 The Heart . . . . . . . . . . . . . . . . . . . . . . . . . . 18

1.6 Excitation-Contraction Coupling Mechanism in Cardiac Cell . . . . . . . . . . 20

1.7 Cardiac tachyarrhythmias in the electrocardiogram . . . . . . . . . . . . 22

1.8 Action Potential Spiral Wave Patterns on Surface of Heart . . . . . . . . . . . . 26

2.1 Phase Singularities on Surface of Heart . . . . . . . . . . . . . . . . . . 36

2.2 Configurations, Gradient Deformation Tensor . . . . . . . . . . . . . . . . . 41

3.1 Rabbit Heart Ultrasound-Scan . . . . . . . . . . . . . . . . . . . . . 58

3.2 Rabbit Heart CT-Scan . . . . . . . . . . . . . . . . . . . . . . . . . . 60

3.3 Mouse Heart OCT-Scan . . . . . . . . . . . . . . . . . . . . . . . . . 63

4.1 Configurations of Particle Systems . . . . . . . . . . . . . . . . . . 75

4.2 Bourguignon Scheme for Anisotropy Control . . . . . . . . . . . . . . . 75

4.3 Reconstructed Rabbit Heart Geometry using Tetrahedral Discretization . . . . . . . . 80

4.4 Fiber Reconstruction . . . . . . . . . . . . . . . . . . . . . . . . . 80

5.1 Electromechanical Waves: Macroscopic Manifestation of Excitation-Contraction Coupling Mechanism in Cardiac Tissue . . . . . . . . . . . . . . . . . . . . 83

5.2 Deformation Measures . . . . . . . . . . . . . . . . . . . . . . . 84

5.3 Planar Electromechanical Wave _. . . . . . . . . . . . . . . . . 86

5.4 Planar Electromechanical Wave . . . . . . . . . . . . . . . . 86

5.5 Elastic Behavior during Trespassing of Electromechanical Wave . . . . . . . . . . . 87

5.6 Two-dimensional Electromechanical Spiral Wave Patterns ～. . . . . . . . . . . . . 89

5.7 Two-dimensional Electromechanical Spiral Wave Patterns ～. . . . . . . . . . . . . . 89

5.8 Two-dimensional Electromechanical Spiral Wave Patterns . . . . . . . . . . . . 90

5.9 Scroll Wave Activity in Elastic Excitable Medium . . . . . . . . . . . . . . . . . . 92

5.10 Scroll Wave Activity in Elastic Excitable Medium: Deformation . . . . . . . . . . . 93

5.11 Single Scroll Wave Rotor in Elastic Excitable Medium . . . . . . . . . . . . . . . . 94

5.12 Scroll Wave and Muscle Fiber Anisotropy . . . . . . . . . . . . . . . . . . . . 94

5.13 Electromechanical Scroll Wave Activity in Heart-Shaped Elastic Excitable Medium . 95

5.14 Turbulent Electromechanical Activity in Heart-Shaped Elastic Excitable Medium . 96

5.15 Reconstruction . . . . . . . . . . . . . . . . . . . 98 
List of Figures

5.16 Reconstruction . . . . . . . . . . . . . . . . . . . . . 99

5.17 Reconstruction Filament Structure . . . . . . . . . . . . . . . . . . . 101

5.18 Reconstruction Filament Structure . . . . . . . . . . . . . . . . . . 101

5.19 Reconstruction Filament Structure . . . . . . . . . . . . . . . . . . . . 102

5.20 Reconstruction Filament Structure . . . . . . . . . . . . . . . . . . . 103

5.21 Association of Co-Localized Electrical and Mechanical Filaments . . . . . . . . . 104

5.22 Electromechanical Filaments . . . . . . . . . . . . . . . . . 106

5.23 Electromechanical Filaments in the Bulk . . . . . . . . . . . . . . . . . 107

5.24 Electromechanical Filaments in the Heart . . . . . . . . . . . . . . . . . . 107

5.25 Imaging Scroll Wave Break-up within Bulk . . . . . . . . . . . . . . . . . . . . 109

5.26 Inverse electromechanical wave imaging . . . . . . . . . . . . . . 110

5.27 Elastic Motion Registration . . . . . . . . . . . . . . . . . . . . . . . 113

5.28 Elastic Motion Registration . . . . . . . . . . . . . . . . . . . . . . . . 114

5.29 Elastic Deformation Tracking with Synthetic Data . . . . . . . . . . . . . . . . 116

5.30 Spiraling Contractile Activity Tracked in Engineered Cardiac Cell Culture Preparation 117

5.31 Spiraling Contractile Activity Tracked in Engineered Cardiac Cell Culture Preparation 117

6.1 Experimental Design . . . . . . . . . . . . . . . . . . . . . . . . 120

6.2 Experimental Setup Schematics . . . . . . . . . . . . . . . . . 121

6.3 Experimental Setup . . . . . . . . . . . . . . . . . . . 122

6.4 Imaging configuration . . . . . . . . . . . . . . . . . . 122

6.5 Reconstruction . . . . . . . . . . . . . . . . . . 123

6.6 LED Switching scheme . . . . . . . . . . . . . . . . . . . . . . . . . 123

6.7 Staining . . . . . . . . . . . . . . . . . . . . . . . 127

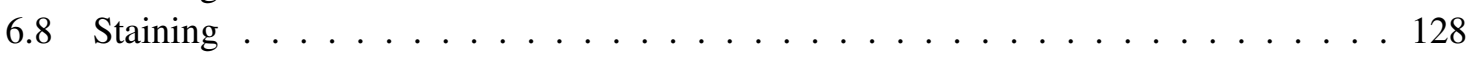

6.9 Tissue Contrast . . . . . . . . . . . . . . . . . . . . . . . . 131

6.10 Raw Fluorescence Video Data . . . . . . . . . . . . . . . . . . 133

6.11 Motion Artifacts . . . . . . . . . . . . . . . . . . . . . . . . 134

6.12 Motion Tracking . . . . . . . . . . . . . . . . . . . . 135

6.13 Raw and Tracked Fluorescence Video Data . . . . . . . . . . . . . . . 136

6.14 Warping . . . . . . . . . . . . . . . . . . . . 137

6.15 Synthetic data . . . . . . . . . . . . . . . . . . . . . . . . . . 139

6.16 Spiral Post-Processing . . . . . . . . . . . . . . . . . . . . . . 140

6.17 Spiral Post-Processing . . . . . . . . . . . . . . . . . . 141

6.18 Imaging Configuration Ultrasound $1 \ldots \ldots$. . . . . . . . . . . . 142

6.19 Imaging Configuration Ultrasound $2 \ldots \ldots \ldots$. . . . . . . . . . . . . . . . . . . . . . . . . . . . . . . . . .

6.20 Ultrasound Speckle Image Data . . . . . . . . . . . . . . . . . . . . . . . 144

6.21 Spiral Post-Processing _ . . . . . . . . . . . . . . . . . . . . 146

6.22 Action Potential Spiral Wave on Ventricular Surface of Rabbit Heart . . . . . . . . 147

6.23 Rotating Elasto-Mechanical Activity on Ventricular Surface of Rabbit Heart . . . . . 147

6.24 Spiral Post-Processing . . . . . . . . . . . . . . . . . . . . . . . . . . . . 149

6.25 Reconstruction of Phase Representations . . . . . . . . . . . . . . . . . . 150

6.26 Phase Representation of Action Potential Spiral Wave on Surface . . . . . . . . . 150

6.27 Phase Representation of Rotating Elasto-Mechanical Rate of Deformation Pattern . . 150

6.28 Co-Localization of Spiral Wave Core and Elastic Topological Defect on Ventricular Surface 
List of Figures

6.29 Electrocardiogram Wash-Out Ventricular Fibrillation and Polymorphic Ventricular Tachycardia . . . . . . . . . . . . . . . . . . . 152

6.30 Calcium Transient Spiral Waves . . . . . . . . . . . . . . . . 153

6.31 Calcium Transient Spiral Waves . . . . . . . . . . . . . . . . . 153

6.32 Electromechanical Coupling during Cardiac Tachyarrhythmias . . . . . . . . . . . . 154

6.33 Spiral Wave-Like Rotating Pattern on Ventricular Surface of Rabbit Heart . . . . . 156

6.34 Phase Representation of Spiral Wave-Like Rotating Pattern on Ventricular Surface of Rabbit Heart . . . . . . . . . . . . . . . . . . . . . . . . . 157

6.35 Spiral Wave-like Deformation Pattern at Midwall . . . . . . . . . . . . . . . 157

6.36 Spiral Post-Processing . . . . . . . . . . . . . . . . . . . 158

6.37 Illustration Scroll Wave Rotor ． . . . . . . . . . . . . . . . . . . . . 159 
List of Figures 


\section{Appendix A}

\section{Physical Units and Abbreviations}

\begin{tabular}{lr}
\hline Symbol & Quantity \\
\hline $\mathbf{A}$ & Surface Area \\
$\mathbf{C}$ & Right Cauchy-Green Deformation Tensor \\
$\mathbf{D}$ & Displacement Vector Field \\
$\mathbf{F}$ & Deformation Gradient Tensor \\
$\mathbf{E}$ & Green-Lagrange Strain Tensor \\
$\lambda(\mathbf{E})$ & Eigenvalue of Green-Lagrange Strain Tensor \\
$\sigma$ & Cauchy Stress Tensor \\
$\mathbf{T}$ & Second Piola-Kirchhoff Stress Tensor \\
$\mathbf{W}$ & Strain Energy Density Function \\
$\mathbb{I}$ & Identity Matrix \\
$\delta$ & Kronecker Delta \\
$\mathbf{u}$ & Displacement Vector \\
$u_{x, y}$ & Components of Displacement Vector \\
$u$ & Excitation Variable \\
$v$ & Refractory Variable \\
$w$ & Refractory Variable \\
$V_{m}$ & Transmembrane Potential \\
$C a_{i}$ & Frequency \\
$f$ & Temperature \\
$T$ & Intracellular Calcium Concentration \\
$T^{a}$ & Ion Concentrations of Intracellular \\
$R$ & and Extracellular Space \\
$e$ & Avogadro Constant \\
$z$ & Aniversal Gas Constant \\
$c_{e} / c_{i}$ & Electron Unit Charge \\
$N_{A}$ & Degree of Ionization \\
\hline &
\end{tabular}

Table A.1: Physical Units 
Appendix A. Physical Units and Abbreviations 
Appendix B. Tables

\section{Appendix B}

\section{Tables}

Table B.1: Ultrasound B-mode acquisition speeds of Visualsonics Vevo 2100 ultrasound imaging system:

\begin{tabular}{ccc}
\hline image width $[\mathrm{mm}$ ] & $\begin{array}{c}\text { framerate [fps] } \\
\text { with 1 focal zone }\end{array}$ & $\begin{array}{c}\text { framerate [fps] } \\
\text { with 2 focal zones }\end{array}$ \\
\hline \hline 2.08 & 1000 & 675 \\
3.08 & 916 & 458 \\
4.08 & 693 & 347 \\
5.08 & 557 & 279 \\
6.08 & 466 & 233 \\
7.08 & 401 & 200 \\
8.08 & 347 & 173 \\
9.08 & 309 & 154 \\
10.08 & 279 & 139 \\
11.08 & 256 & 127 \\
12.08 & 233 & 117 \\
13.08 & 215 & 108 \\
14.08 & 202 & 100 \\
\hline
\end{tabular}

for following imaging parameters: image depth $10 \mathrm{~mm}$, power: $100 \%$, gain: $22 \mathrm{~dB}$, line density: standard, persistence: off, sensitivity: standard, dynamic range: $65 d B$ 


\begin{tabular}{ccc}
\hline \hline Parameter & Spiral & Turbulence \\
\hline$g_{f i}$ & 5.6093115 & 4.0 \\
$\tau_{r}$ & 62.41 & 28.0 \\
$\tau_{s i}$ & 63.4124 & 29.0 \\
$\tau_{0}$ & 8.36 & 12.5 \\
$\tau_{v}^{+}$ & 10.8037 & 3.33 \\
$\tau_{v 1}^{-}$ & 40.4284 & 2.0 \\
$\tau_{v 2}^{-}$ & 36.5891 & 15.0 \\
$\tau_{w}^{+}$ & 663.715 & 670.0 \\
$\tau_{w}^{-}$ & 132.373 & 61.0 \\
$u_{c}$ & 0.13 & 0.13 \\
$u_{v}$ & 0.05 & 0.05 \\
$u_{c}^{s i}$ & 0.45 & 0.45 \\
\hline \hline
\end{tabular}

Table B.2: Parameters used in this work for Fenton-Karma model given by equations 2.1.17-2.1.22 for two different regimes: the spiral regime and the turbulent regime 


\section{Additional Comments}

This thesis contains phrasings and excerpts from my Diplom-thesis ${ }^{199}$ in chapters 1, 2 and 3 during the description of introductory and basic methodological backgrounds. 



\section{Acknowledgements}

Foremost, I would like to thank my advisor Stefan Luther for introducing me to the topic and giving me the opportunity to explore a new, exciting field that proved to be very rewarding. As a PhDstudent, one could not ask for a better advisor.

This thesis has received contributions from colleagues and students, whom I would like to thank. I would like to thank Mohammed Chebbok, who showed immediate interest in the topic and was very supportive from the very beginning and with whom I have conducted most of the experiments. I would like to thank Sebastian Stein who was a very motivated Master student, working together with me on computational modeling during the time of my $\mathrm{PhD}$ and who enhanced the numerical methods significantly. I would like to thank Claudia Richter who has contributed to this work with her cell culture preparations. I would like to thank Marion Kunze who has helped to prepare the experiments. I would like to thank Daniel Hornung who has helped me to perform the computerized tomography scans. I would like to thank Ilja Uzelac and Flavio Fenton from Georgia-Tech, Atlanta, who have built and provided a fast-switching diode driver for the multi-parametric fluorescence imaging experiments.

I would like to thank the two further members of my Thesis Committee, Stephan Herminghaus and Sarah Köster, and the other members of the Examination Board, Ulrich Parlitz, Jens Frahm and Eberhard Bodenschatz for taking the time to revise my work and to give me valuable feedback during the development of this work.

I would like to thank the entire Max Planck Research Group Biomedical Physics at the Max Planck Institute for Dynamics and Self-Organization, namely, Johannes Schröder-Schetelig, Tariq Baig, Philipp Bittihn, Alexander Schlemmer, Amgad Squires, TK Shajahan, Valentin Krinsky, Edda Boccia, Sebastian Berg, Gisa Luther, Hendrik tom-Wörden, Jan Schumann-Bischoff, Thomas Lilienkamp and Nour Raad for being such great and supportive colleagues.

I wish to thank Lindsay Bess for the many hours that she spent patiently watching me sit in front of my computer and for giving me advice and general support and who helped me to improve my knowledge of the English language. Last, but of course not the least, I would like to thank my parents who supported my decision to study, supported me financially during the studies and without whom I would never have been able to obtain a degree that enabled me to pursue my doctoral studies.

\section{Funding}

This research has received funding from the Collaborative Research Centre 1002: 'Modulatory Units in Heart Failure' (Sonderforschungsbereich 1002: 'Modulatorische Einheiten bei Herzinsuffizienz') and the Max Planck Society. 